Old Dominion University

ODU Digital Commons

Electrical \& Computer Engineering Theses \&

Dissertations

Electrical \& Computer Engineering

Winter 2006

\title{
Stability Analysis of Hybrid Jump Linear Systems With Markov Inputs
}

Arturo Tejada Ruiz

Old Dominion University

Follow this and additional works at: https://digitalcommons.odu.edu/ece_etds

Part of the Electrical and Computer Engineering Commons

\section{Recommended Citation}

Ruiz, Arturo T.. "Stability Analysis of Hybrid Jump Linear Systems With Markov Inputs" (2006). Doctor of Philosophy (PhD), Dissertation, Electrical \& Computer Engineering, Old Dominion University, DOI:

10.25777/genx-e078

https://digitalcommons.odu.edu/ece_etds/189

This Dissertation is brought to you for free and open access by the Electrical \& Computer Engineering at ODU Digital Commons. It has been accepted for inclusion in Electrical \& Computer Engineering Theses \& Dissertations by an authorized administrator of ODU Digital Commons. For more information, please contact digitalcommons@odu.edu. 


\title{
STABILITY ANALYSIS OF HYBRID JUMP LINEAR SYSTEMS WITH MARKOV INPUTS
}

by

\author{
Arturo Tejada Ruiz
}

B.S. December 1996, Pontificia Universidad Católica del Perú

M.S. August 2002, Old Dominion University

A Dissertation Submitted to the Faculty of Old Dominion University in Partial Fulfillment of the

Requirement for the Degree of

DOCTOR OF PHILOSOPHY

ELECTRICAL AND COMPUTER ENGINEERING

OLD DOMINION UNIVERSITY

December 2006

Approved by:

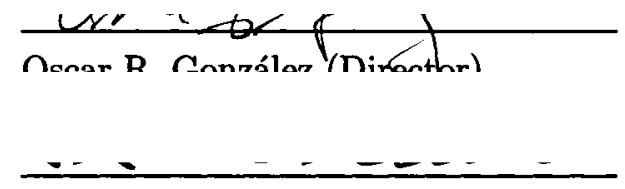

Victor A. Carreño

N. Rao Chaganty

W. Steven Gray

Vishpu K. Lakdawála (Chairman) 


\title{
ABSTRACT \\ STABILITY ANALYSIS OF HYBRID JUMP LINEAR SYSTEMS WITH MARKOV INPUTS
}

\author{
Arturo Tejada Ruiz \\ Old Dominion University, 2006 \\ Director: Dr. Oscar R. González
}

In the past two decades, the number of applications that make use of supervisory algorithms to control complex continuous-time or discrete-time systems has increased steadily. Typical examples include air traffic management, digital control systems over networks, and flexible manufacturing systems. A common feature of these applications is the intermixing of the continuous dynamics of the controlled plant with the logical and discrete dynamics of the supervising algorithms. These so-called $h y$ brid systems are the focus of much ongoing research. To improve the performance of these systems, it is important to analyze the interactions between the supervising algorithms and the plant. Few papers have studied this interaction when the plant is represented by a discrete-time system. Thus, this dissertation fixes this deficiency by addressesing the following three main objectives: to introduce a new modeling framework for discrete-time stochastic hybrid systems suitable for stability analysis; to derive testable stability conditions for these models; and to demonstrate that these models are suitable to study real-world applications. To achieve the first objective, the Hybrid Jump Linear System model is introduced. Although it has many of the same modeling capabilities as other formalisms in the literature (e.g., Discrete Stochastic Hybrid Automata), it possesses the unique advantage of representing the dynamics of both the controlled plant and the supervising algorithm in the same analytical framework: stochastic difference equations. This enables the study of their joint properties such as, for example, mean square stability. The second objective is addressed by developing a collection of testable sufficient mean square stability conditions. These tests are developed by applying, successively, switched systems' techniques, singular value analysis, a second moment lifting technique, and Markov kernel methods. The final objective is achieved by developing a hybrid jump linear system model of an AFTI-F16 flight controller deployed on a fault tolerant computer with rollback and cold-restart capabilities, and analyzing its stability properties. 
Copyright (C) 2006, by Arturo Tejada Ruiz. All Rights Reserved 


\section{ACKNOWLEDGEMENTS}

I would like to express my gratitude to Dr. Oscar R. González for guiding me through this important period of my life. His exemplary professionalism was an inspiration and a motivation to complete this research. He also reminded me that family is above anything else.

I will be always indebted to Dr. W. Steven Gray. His wit, warmth and unfailing dedication to research lifted my spirit many times. I will especially miss our long conversations and Colley Avenue's Philosophical Society.

I also want to recognize and thank Dr. Victor Carreño and Dr. Rao Chaganty for taking upon themselves the task of reading my dissertation. Their views and ideas have certainly enriched my research.

I am very grateful to my family and friends for their support during the years it took to complete this work. I especially want to thank Heber, whose deep knowledge of mathematics never ceased to amaze me and propelled me forward in new directions; Jose, a larger than life figure and a source of great moments; Anushka, my little sister and partner in crime; and Vessi, my partner in life.

Finally, I wish to acknowledge the support of the NASA Langley Research Center through their grants NNL04AA03A and NCC-1-03026. 


\section{DEDICATION}

To Margarita, Urbano, Vessi, Jose, Anushka, Heber, Anamaria, Hong, Yaneth, Mayling, and all those that stubbornly supported me (although they knew better...) 


\section{TABLE OF CONTENTS}

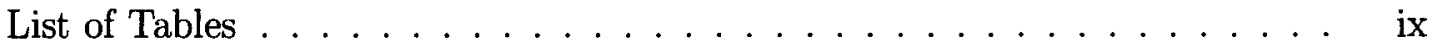

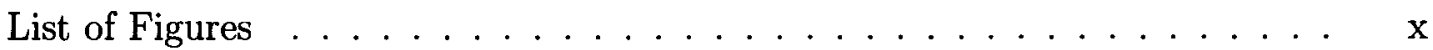

List of Acronyms ................... . . . . . . .

\section{CHAPTERS}

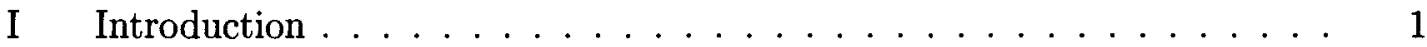

I.1 Background, Motivation, and Related Literature . . . . . . . . . . . 4

I.2 Dissertation Goal and Objectives . . . . . . . . . . . . . . . . 10

I.3 Dissertation Overview . . . . . . . . . . . . . . . . . 10

I.4 A Word on Notation . . . . . . . . . . . . . . . . . . . . 12

II The Hybrid Jump Linear System Model . . . . . . . . . . . . . . . . 15

II.1 Introduction . . . . . . . . . . . . . . . . . . . 15

II.2 The Hybrid Jump Linear System Model . . . . . . . . . . . . . . 16

II.2.1 Finite State Machines . . . . . . . . . . . . . . . . 16

II.2.2 The Supervisor . . . . . . . . . . . . . . . . . 18

II.2.3 The Jump Linear Closed-Loop System . . . . . . . . . . . . 20

II.2.4 Formal Definition of a HJLS . . . . . . . . . . . . . . . 21

II.2.5 Canonical Hybrid Jump Linear Systems . . . . . . . . . . . . 21

II.3 Hybrid Jump Linear Systems with Performance Supervision . . . . . 23

II.3.1 Hybrid Jump Linear Systems with Performance Supervision . 24

II.3.2 Hybrid Jump Linear Systems with Performance Map . . . . . 25

II.4 Chapter Summary and Conclusions . . . . . . . . . . . . . . . 26

III Stability Analysis of Hybrid Jump Linear Systems . . . . . . . . . . . . 28

III.1 Introduction . . . . . . . . . . . . . . . . . . . . . . . 28

III.2 Stability Analysis Framework . . . . . . . . . . . . . . . . . . 30

III.2.1 Stability Concepts . . . . . . . . . . . . . . . . . 33

III.3 Stability Analysis of HJLS's Using the Switched Systems Approach 37

III.3.1 Preliminaries . . . . . . . . . . . . . . . . . . . . . . 37

III.3.2 Stability Analysis . . . . . . . . . . . . . . . . . . . 39

III.4 Stability Analysis of HJLS's Using Singular Value Techniques . . . . 45

III.4.1 Stability Analysis of a Simple HJLS with Performance Supervision 46

III.5 Stability Analysis of HJLS's Using a Lifting Technique . . . . . . . . 56

III.5.1 Mean Square Stability of HJLS's with Performance Map . . . 57

III.5.2 Mean Square Stability of JLS's Driven by General Discrete-

Time, Finite-State Stochastic Processes . . . . . . . . . . 62

III.6 Numerical Examples . . . . . . . . . . . . . . . . . . . . . . 74

III.6.1 Example 1: HJLS with Performance Map . . . . . . . . . . . 74

III.6.2 Example 2: A JLS Driven by a Stochastic Automaton . . . . . 76

III.7 Chapter Summary and Conclusions . . . . . . . . . . . . . . 78 
IV Stability Analysis of HJLS's Using the Markov Kernel Technique . . . . 81

IV.1 Introduction . . . . . . . . . . . . . . . . . . . 81

IV.1.1 Basic Properties of Markov Chains in Metric Spaces . . . . . . 83

IV.2 The Markov Property of Non-Linear Difference Equations with Markovian Inputs . . . . . . . . . . . . . . . . . . . . 86

IV.2.1 The Markov Property of Difference Equations with Markovian Inputs . . . . . . . . . . . . . 87

IV.2.2 The Markov Kernel of Non-Linear Difference Equations with Markovian Inputs . . . . . . . . . . . . . . 92

IV.3 The Markov Property of Hybrid Jump Linear Systems . . . . . . . . 94

IV.3.1 Markov Kernels Associated with FSM's . . . . . . . . . . . . . 95

IV.3.2 On the Markov Property and Markov Kernel of Canonical HJLS's 99

IV.3.3 Probability Distributions Associated with HJLS's . . . . . . . 101

IV.4 Sufficient MS Stability Tests for Canonical HJLS's . . . . . . . . . . . 106

IV.4.1 MS Stability Tests Based on Common Invariant Subspaces . . 106

IV.4.2 MS Stability Tests Based on Theorem III.5.2 . . . . . . . . . . 111

IV.5 Chapter Summary and Conclusions . . . . . . . . . . . . . . . . 122

V Simulation Example . . . . . . . . . . . . . . . . . . . . 124

V.1 Introduction . . . . . . . . . . . . . . . . . . . . . . . . 124

V.2 On Fault Recovery Mechanisms and Their Dynamical Models . . . . 125

V.2.1 Motivation and Background . . . . . . . . . . . 125

V.2.2 Dynamical Models of Digital Control Systems Deployed in Fault

Recoverable Computers . . . . . . . . . . . . . . . 127

V.3 An Advanced Fault Recovery Mechanism . . . . . . . . . . . . . . . . 129

V.4 Stability Analysis of the Advanced Recovery Mechanism . . . . . . . 132

V.4.1 Theoretical Stability Analysis . . . . . . . . . . . . . 132

V.4.2 Monte Carlo Simulations . . . . . . . . . . . . . . . . . . 136

VI Conclusions and Future Research . . . . . . . . . . . . . . . . 140

VI.1 Conclusions . . . . . . . . . . . . . . . . . . . . . 140

VI.2 Future Research . . . . . . . . . . . . . . . . . . . . . . . . 141

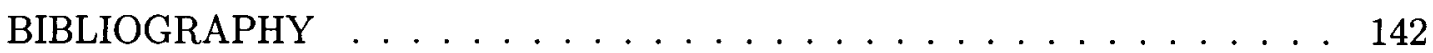

\section{APPENDICES}

A Some Elements from Measure Theory . . . . . . . . . . . . . . 150

A.1 Basic Conventions and Definitions . . . . . . . . . . . . . 150

A.2 Some Properties of $\sigma$-Algebras . . . . . . . . . . . . . . . . 151

A.2.1 Structure . . . . . . . . . . . . . . . 151

A.2.2 Combination . . . . . . . . . . . . . . . 152

A.2.3 Simplification of $\sigma$-Algebras . . . . . . . . . . . . . . 154

A.3 On Independence and Expected Values . . . . . . . . . . . . . . . 155

A.3.1 Independence . . . . . . . . . . . . . . . . 155 


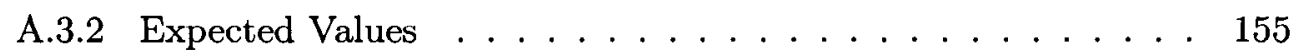

B Proof of Theorems IV.2.2 and IV.2.4 . . . . . . . . . . . . . 158

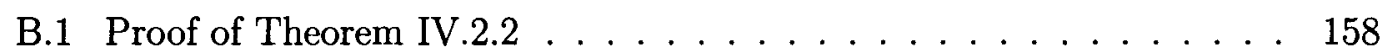

B.1.1 Proof of Fact (i) . . . . . . . . . . . . . 159

B.1.2 Proofs of Facts (ii) and (iii) . . . . . . . . . . . 160

B.1.3 Proof of Theorem IV.2.2 . . . . . . . . . . . . . . 162

B.2 Proof of Theorem IV.2.4 . . . . . . . . . . . . . . . . . . 163

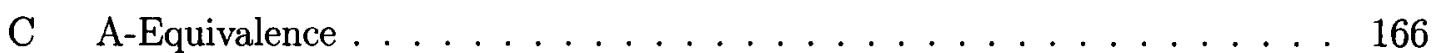

C.1 Introduction . . . . . . . . . . . . . . . . 166

C.2 A-Equivalence of HJLS's . . . . . . . . . . . . . . . . . . . . . 167

C.3 A-Equivalence of General Moore-Type HJLS's and Canonical HJLS's 170

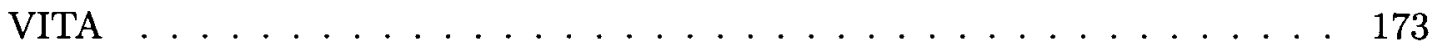




\section{LIST OF TABLES}

Page

I Hybrid Jump Linear Systems' Parameters. . . . . . . . . . . . . . . . 75

II Stability Conditions Satisfied by the HJLS. . . . . . . . . . . . 76

III Summary of The Main Results in Chapter III. . . . . . . . . . . . . . 80

IV Summary of Main Results in Chapter IV. . . . . . . . . . . . . . . . 122 


\section{LIST OF FIGURES}

Page

1 Block diagram of a simplified hierarchical embedded control system with a jump linear system. . . . . . . . . . . . . . . 2

2 A hybrid jump linear system. . . . . . . . . . . . . . . 3

3 An advanced model of a closed-loop system deployed on a fault-tolerant

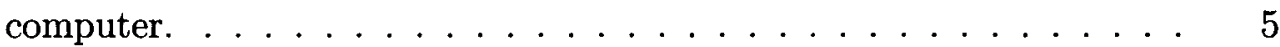

4 A general HJLS. . . . . . . . . . . . . . . . 20

5 A canonical HJLS . . . . . . . . . . . . . . . . 20

6 A HJLS with performance supervision. . . . . . . . . . . . . 24

7 A HJLS with performance map. . . . . . . . . . . . . . 24

8 The organization of the main results in this chapter. . . . . . . . 30

9 Transition diagram for a finite state machine representation of a logical OR operation. . . . . . . . . . . . . . . . . . 49

10 Structure of $\hat{\Pi}_{\varphi}$, the permuted transition probability matrix of $\varphi(k) . \quad 70$

11 Jump linear system driven by a stochastic automaton. . . . . . . . . . 71

12 Mean of the norm squared (HJLS 1) and second moment of $\boldsymbol{x}(k)$ (HJLS's 2 and 3). HJLS 1 satisfies Theorem III.3.3 while HJLS 2 satisfies Theorem III.5.1. HJLS 3 satisfies neither condition. . . . . . 77

13 Estimate of $\mathbf{E}\left\{\|\boldsymbol{x}(k)\|^{2}\right\}$ computed via 10,000 Monte Carlos runs. . . 78

14 Spectral radius of $\mathcal{A}$ as a function of $p_{0,0}^{N}$ and $p_{1,1}^{N}$ (see (59)). . . . . 79

15 Digital closed-loop system under consideration. . . . . . . . . . . . 128

16 The example's FSM structure. Inside each circle, $e_{i}$ represents the current state and output symbol. The labels over the arcs represent the value of the FSM's inputs, $(N(k), \nu(k))$, that triggers the state transition (the $x$ represents a don't care condition). . . . . . . . 132

17 The restricted operation of the supervisor when $\alpha=\infty$. The labels over the arcs show the value of $\boldsymbol{N}(k)(\boldsymbol{\nu}(k)=0$ for all $k \geq 0)$. . . 134

18 The restricted operation of the supervisor when $\alpha=0$. The labels over the arcs show the value of $N(k)(\boldsymbol{\nu}(k)=1$ for all $k \geq 0) \ldots \ldots 134$

19 Digital jump linear closed-loop system considered for Monte Carlo simulations. . . . . . . . . . . . . . . . . . . . 136

20 Top: Spectral radius of $\mathcal{A}_{M_{z}}, \mathcal{A}_{i i d_{\mathrm{CR}}}$, and $\mathcal{A}_{i i d_{\mathrm{RB}}}$ as a function of $p_{0}^{N}=$ $\operatorname{Pr}\{N(k)=0\}$. Bottom: A magnification of the region $0.9998 \leq p_{0}^{N} \leq 1.138$

21 Monte Carlo simulation results. Each simulation comprised 500 runs of 1000 seconds. In both cases, $p_{0}^{N}=0.99994, z_{0}=e_{1}$, and $x_{0}=$ $\left[\begin{array}{lllllllllll}0 & 1 & 0 & 0 & 0 & 0 & 0 & 0 & 0 & 0 & 0\end{array}\right]^{\mathrm{T}}$. In the top plot $\alpha=0$, while in the bottom plot $\alpha=1000 \ldots \ldots \ldots \ldots \ldots \ldots$ 


\section{LIST OF ACRONYMS}

Page

$A / S \quad$ Analog to Symbol Map ..................... 3

ARM Advanced Recovery Mechanism . . . . . . . . . . . . 124

asMJLS Auxiliary Scalar Markov Jump Linear System . . . . . . . . . . . 49

CMF Common Mode Faults . . . . . . . . . . . . . . 125

DES Discrete Event System . . . . . . . . . . . . . 8

DHA Discrete Hybrid Automata . . . . . . . . . . . . 9

DHSA Discrete Hybrid Stochastic Automata . . . . . . . . . . 3

FSM Finite State Machine . . . . . . . . . . . . . 3

HJLS Hybrid Jump Linear System . . . . . . . . . . . . . . 2

i.i.d. Independent and Identically Distributed Process _. . . . . . . 87

JLS Jump Linear System . . . . . . . . . . . . . . . . . . 1

MJLS Markov Jump Linear System . . . . . . . . . . . . . . 7

MLD Mixed Logical Dynamical (System) . . . . . . . . . . . 9

MSS Mean Square Stability .................. 29

PDP Piecewise Deterministic Markov Process . . . . . . . . . . . . 3

PWA Piecewise Affine (System) $\ldots \ldots \ldots \ldots$ 


\section{CHAPTER I}

\section{INTRODUCTION}

A general modeling framework for embedded control systems is a hybrid system representation which allows the inclusion of analog and digital subsystems together with a model for the interfaces between them and the inherent constraints on their states and independent variables. These representations have the sufficient fidelity that is needed in the analysis and design of safety critical systems such as reliable, fault-tolerant control systems that can affect human lives. A particular advantage of hybrid systems is that they can directly represent hierarchical control systems, including the dynamics of a decision making supervisor present in most embedded control systems (cf. DeCarlo, Branicky, Petterson \& Lennartson (2000), Henzinger (1996), Koutsoukos, Antsaklis, Stiver \& Lemmon (2000)). In the presence of noise, disturbances or uncertainties, such hierarchical embedded control systems can be represented as in Figure 1, where the low-level closed-loop dynamics are represented by a Jump Linear System (JLS) that is switched by $\{\boldsymbol{\theta}(k), k \geq 0\}$ and $\left\{N_{l}(k), k \geq 0\right\}$, namely the decisions of the supervisor, $\boldsymbol{\theta}(k)$, and the value of a stochastic process, $N_{l}(k)$, which represents, for instance, the state of a fault or internal event detector. The switching is used to model, for example, the change in closed-loop dynamics for different operating regions or the change in closed-loop dynamics between normal and recovery operation during an upset or critical failure. This model is useful for studying the effects of the high-level supervisor on the low-level control loop

dynamics where the supervisor takes decisions based on the states of the JLS and 


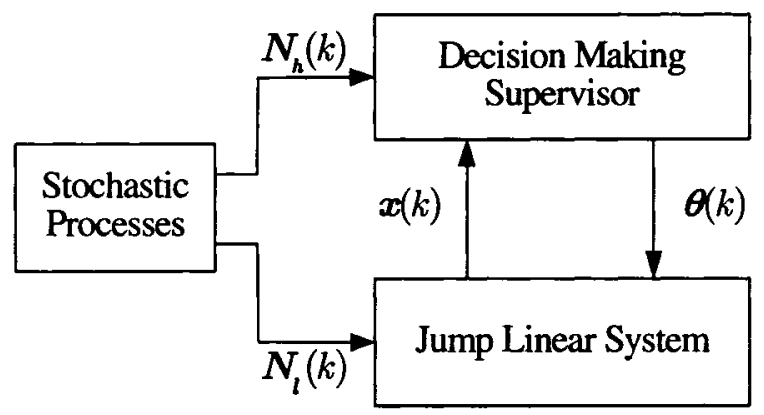

FIG. 1: Block diagram of a simplified hierarchical embedded control system with a jump linear system.

stochastic process $\left\{\boldsymbol{N}_{\boldsymbol{h}}(k), k \geq 0\right\}$. The state dependency allows the supervisor to command a control law change or to select among a set of fault-tolerant recovery techniques. In addition, the supervisor can monitor and make decisions based on the performance of the low-level control system. The stochastic process $\left\{\boldsymbol{N}_{\boldsymbol{h}}(k), k \geq 0\right\}$ typically models the status of environmental sensors that indicate the presence of harsh environmental exogenous conditions. In addition to the interpretation given above, $\left\{N_{l}(k), k \geq 0\right\}$ can also be used to represent noisy sensor inputs or commands, component failure detection, etc. Likewise $\left\{N_{h}(k), k \geq 0\right\}$ can be used to represent high-level fault processes (e.g. machine breakdowns), random variation of requirements (e.g. increase/deacrease of production goals), etc. Clearly, the inclusion of these stochastic processes enhances the fidelity of hybrid system models by taking into account stochastic uncertainty.

For qualitative and quantitative analysis, a particular type of jump linear system and decision making supervisor will be considered as shown in Figure 2. These models, called Hybrid Jump Linear System (HJLS), are composed of a Finite State Machine 


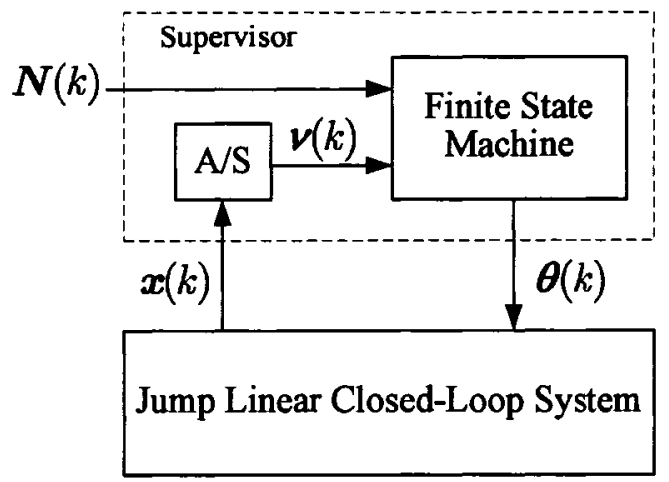

FIG. 2: A hybrid jump linear system.

(FSM), a state feedback analog-to-symbol $(A / S)$ map, and a jump linear closed-loop system. The discrete-time stochastic process $\{\boldsymbol{\theta}(k), k \geq 0\}$ that drives the jump linear closed-loop system is generated by the finite state machine, which represents the decision making process of the high-level supervisor. The supervisor itself is driven by a (feedback) function of the JLS's $n$-dimensional state vector, $\nu(k)$, and by a stochastic process $\{N(k), k \geq 0\}$. This particular configuration was devised for practical and theoretical reasons. On the one hand, hybrid jump linear systems can be used to extend the research, initiated by Zhang, Gray \& González, on the analysis of the effects of harsh neutron environments on closed-loop system performance, which is of interest in aviation safety (see Zhang, Gray \& González 2005). On the other hand, these systems are of theoretical interest. They are related to several main stream hybrid models in the literature, such as Discrete Hybrid Stochastic Automata (DHSA) and Piecewise Deterministic Markov Processes (PDP) (Davis 1993, Bemporad \& Di Cairano 2005), whose stability characteristics have not been investigated with the tools to be introduced in this dissertation. 
The rest of the chapter is organized as follows. Section I.1 provides the motivation and background that led to the development of the hybrid jump linear system formalism. It also summarizes, briefly, the literature related to this class of systems. Section I.2 gives a precise formulation of the goal and objectives that this dissertation addresses. Section I.3 provides an overview of the dissertation, including the methodology used to pursue the objectives described in Section I.2. Finally, Section I.4 summarizes the notation used throughout the dissertation.

\section{I.1 BACKGROUND, MOTIVATION, AND RELATED LITERATURE}

\section{Motivation for the Hybrid Jump Linear System Formalism}

During the past ten years, the Systems Research Laboratory at Old Dominion University (http://www.srl.odu.edu/) has conducted research on the system-level effects of harsh electromagnetic environments on digital flight controllers. Commercial aircraft are exposed to these environments when, for instance, they fly through thunderstorms or approach radar ground stations (Hess 1997). Although these conditions do not usually pose a significant safety hazard for older aircraft, it has been documented that newer fly-by-wire models can be severely affected (Malekpour \& Torres 2000, Hess \& Belcastro 2001, Belcastro 1997). The computer platforms onboard newer aircraft include an array of fault-tolerant provisions. Of particular interest were those computers designed and built to be resilient not only to localized faults but also to pervasive faults. Pervasive faults, better known as common-mode faults, cannot be tolerated with standard hardware redundancy provisions. They are handled with information redundancy techniques, such as rollback recovery, which 


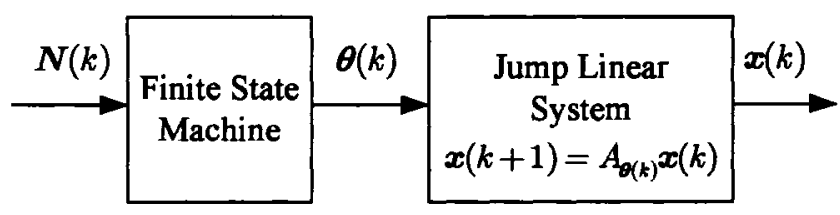

FIG. 3: An advanced model of a closed-loop system deployed on a fault-tolerant computer.

require one to flush and replace the (corrupted) data stored in the memory of the affected computer (see (Tejada 2002) for a detailed account of these ideas). During the recovery procedure the faulty computer is rendered inoperative, which suspends, for a brief period, the control law computations. The net effect is that the aircraft's dynamics are changed, abruptly, from a closed-loop configuration to an open-loop configuration (and vice-versa). As was argued in (Gray, González \& Dǒgan 2000), a good model for this behavior is given by

$$
\boldsymbol{x}(k+1)=A_{\boldsymbol{N}(k)} \boldsymbol{x}(k),
$$

where $\{N(k), k \geq 0\}$ is a two-state stochastic process (usually a Markov chain) which represents the presence $(\boldsymbol{N}(k)=1)$ or absence $(\boldsymbol{N}(k)=0)$ of computer faults, and $A_{N(k)} \in \mathbb{R}^{n \times n}$ are the respective closed-loop state matrices. Thus, at every time $k \geq 0$, $\boldsymbol{N}(k)$ determines the operating mode of the system by selecting an appropriate state matrix. As discussed in (Tejada 2002), the structure of the matrices can be devised to represent the closed-loop dynamics of the aircraft $\left(A_{0}\right)$, and the dynamics of the recovery process $\left(A_{1}\right)$.

The model discussed above lacks the ability to easily control the duration of the recovery procedure. This problem was solved in (Patilkulkarni, Herencia-Zapana, Gray \& González 2004) by introducing a finite state machine (or a stochastic finite 
state automaton (Zhang et al. 2005)), which reacted to the fault process, $\{N(k), k \geq$ $0\}$, by generating a new, precisely timed mode-switching signal $\{\boldsymbol{\theta}(k), k \geq 0\}$. This extended model, depicted in Figure 3, was very successful not only because its stability and performance could be studied with well established tools in the literature (cf. Costa, Fragoso \& Marques 2005), but also because it properly captured the essence of the fault-recovery phenomena under study (Zhang et al. 2005).

It is possible, however, to find situations for which this extended model is not entirely suitable. For example, an advanced recovery logic could be implemented to decide, based on the conditions of the closed-loop system and on the conditions of the computer platform, which recovery procedure is to be executed. Since different procedures require different amounts of resources (computational power, recovery time, electrical power, etc.), the recovery logic could choose to execute a simpler and less resource-consuming recovery procedure when the closed-loop system exhibits "good performance", or even to not execute a recovery operation. On the other hand, when the performance is especially poor, the recovery logic could execute a more extensive and resource-consuming recovery procedure.

Another example where the extended model falls short is where a gain scheduling controller is deployed on a fault-tolerant computer. Essentially, a gain scheduling controller is composed of two elements: an array of standard closed-loop controllers, and a selection mechanism. This mechanism activates one closed-loop controller at a time based on, for example, the location of the closed-loop system's state vector, $\boldsymbol{x}(k)$, in the phase space. Thus, when a gain scheduling controller is deployed on a fault-tolerant computer, the dynamics of the closed-loop system are changed based 
on both the value of $\boldsymbol{x}(k)$ and on the presence of computer faults.

In these two examples, the computer executing the control law is also running an algorithm which generates the mode changes based on closed-loop system information. Clearly, this behavior could not be captured with the model in Figure 3. For this it is necessary to include a state feedback loop. This gave rise to the hybrid jump linear system model in Figure 2. It also marked a departure from previous work because in general the process $\{\boldsymbol{\theta}(k), k \geq 0\}$ driving the jump linear system is not a Markov chain and its statistics (e.g., distribution functions) depend on the state process $\{\boldsymbol{x}(k), k \geq$ 0\}. Consequently, the tools available for Markov Jump Linear Systems (MJLS's) that were used in (Tejada 2002, Zhang et al. 2005) to analyze the stability and performance of the extended model in Figure 3, could not be applied to study HJLS's. Hence, a new framework for stability and performance analysis was needed. This dissertation aims to provide such a framework and to derive testable mean square stability conditions for HJLS's. Due to the similarities between HJLS's and MJLS's, several of the stability concepts and conditions derived for hybrid jump linear systems were adapted from the literature on MJLS's. In particular, the emphasis in this dissertation lies on mean square stability, which is necessary to design optimal linear quadratic regulators (Costa et al. 2005). A complete mean square stability analysis is also necessary to extend the performance analysis in (Zhang et al. 2005) to hybrid jump linear systems. The stability analysis presented here is important not only for the benefit of the faulttolerant community but also for the hybrid system community at large, due to the aforementioned connection between this model and other established hybrid models in the literature. This connection is explored next. 


\section{Related Hybrid System Models}

In Figure 2, the finite state machine represents the supervisor's decision making algorithm. In general, an algorithm can be more complex than a set of conditional (IF-THEN-ELSE) statements. It could also include, for example, decisions based on time, order, repetition, or security considerations. Moreover, the algorithm could also react to both synchronous and asynchronous "events". Clearly, a finite state machine is insufficient to represent a general decision making algorithm. If necessary, the supervisor could be represented by a more complex automaton (Antsaklis, Koutsoukos \& Zaytoon 1998), leading to a more abstract supervisory system setup such as the one in Figure 1.

Supervisory systems have been studied in the context of hybrid systems by several investigators. For instance, (Koutsoukos et al. 2000) presented a detailed analysis of a supervisory control system based on a hybrid system model. It was shown that the closed-loop system and its interfaces (e.g., the $A / S$ map) can be modeled with a Discrete Event System (DES). By also modeling the supervisor with a discrete event system, the authors reduced the supervisory control problem to that of designing a DES controller. DES are easily analyzed to determine properties such as safety or liveliness, but are not as suitable to analyze, for example, stability. As a consequence, several other formalisms and techniques have been developed to investigate different aspects of hybrid systems. An excellent survey of these models and techniques can be found in (Antsaklis et al. 1998). As pointed out there, hybrid models range from those tailored to study properties such as stability, robustness, and optimal control 
(Ye \& Michel 1998, Davis 1993) to those specifically suited to address the properties of real-time embedded systems (Henzinger 1996). There are other models of interest located in between these extremes. Of particular interest are Piecewise Affine (PWA) systems (Sontag 1981), Mixed Logical Dynamical (MLD) systems (Bemporad \& Morari 1999), and Discrete Hybrid Automata (DHA) (Torrisi \& Bemporad 2004), which are known to be equivalent and suitable for modeling, simulation, and verification of a broad range of hybrid applications (Torrisi \& Bemporad 2004, Heemels, Schutter \& Bemporad 2001).

Deterministic hybrid jump linear systems, that is, HJLS's where $\{N(k), k \geq 0\}$ has been replaced by deterministic discrete-time signals, are the autonomous version $\left(u_{r}(k)=0\right)$ of DHA. However, unlike DHA's, the stability of deterministic HJLS's can be directly studied with the tools to be be presented in this dissertation (DHA's need to be translated first into the PWA formalism). As stochastic models, HJLS's are the autonomous version of Discrete Hybrid Stochastic Automata (DHSA), the stochastic extension of DHA (Bemporad \& Di Cairano 2005). The stability analysis of these models, which have been recently introduced, is still an open problem. Moreover, as explained in (Bemporad \& Di Cairano 2005), DHSA are known to be the discretetime version of piecewise deterministic Markov processes (PDP). The latter have been extensively studied due to their ability to model a broad range of phenomena. The stability of PDP's, however, has not yet been studied with the second moment techniques presented here. Thus, the tools and results presented in this dissertation constitute both a significant contribution to the study of DHSA and provide a seed for a new viewpoint in the stability analysis of PDP's. 


\section{I.2 DISSERTATION GOAL AND OBJECTIVES}

The goal of this dissertation follows.

Dissertation Goal: To introduce a new model class for discrete-time stochastic hybrid systems that combine continuous and symbolic dynamics. The model class should be amenable for stability analysis and useful to study advanced fault-tolerant recovery procedures.

To attain this goal, the following objectives will be addressed.

1. To introduce the general hybrid jump linear system model class shown in Figure 2 and three different subclasses: canonical HJLS's, HJLS's with performance supervision, and HJLS's with performance map.

2. To develop a mean square stability analysis framework for hybrid jump linear systems.

3. To develop testable mean square stability conditions using four different approaches: switch systems techniques, singular value analysis, a second moment lifting technique, and a Markov kernel technique.

4. To develop a HJLS model and analyze the mean square stability of an AFTIF16 flight controller deployed on a fault-tolerant computer platform equipped with an advance fault recovery mechanism.

\section{I.3 DISSERTATION OVERVIEW}

The research developed in this document is based on the methods and tools of (Ye \& Michel 1998, Costa et al. 2005, Ji \& Chizeck 1990a), which were adapted to attain the dissertation's objectives. The first three objectives are addressed in Chapters 
II-IV. In Chapter II, the hybrid jump linear system model is presented in detail. Four different versions are discussed: general HJSL, canonical HJLS, HJLS with performance supervision, and HJLS with performance map. Each of these variations has unique advantages either from a modeling perspective or from a stability analysis point of view.

Stability analysis of HJLS's is done in Chapters III and IV. In Chapter III, an appropriate stability analysis framework is introduced. Then, the mean square stability analysis of HJLS's is explored by applying in succession switched systems techniques, singular value analysis, and a second moment lifting technique. Several testable sufficient mean square stability conditions are developed for the various classes of HJLS's. The main result of this chapter is Theorem III.5.2, which is used to derive a sufficient mean square stability test for HJLS's with performance map. This test is later extended to canonical HJLS's in Chapter IV. The results are arranged in decreasing order of conservativeness and increasing order of generality. Each is illustrated with a simple numerical example.

In Chapter IV, hybrid jump linear systems are analyzed using Markov chain techniques. It is first proven that the state of a HJLS realizes a Markov chain in a metric space. Then, the associated Markov kernel is used to derive a closed-form formula for the evolution of the distribution of the state vector, $\mu_{k}^{y}$. This formula is used to derive two sets of stability results based on a structural constraint imposed over the supervisor and using Theorem III.5.2. The most important result of this chapter is Theorem IV.4.4, which provides a simple testable condition for the mean square stability of canonical HJLS's. 
The fourth objective of this dissertation is addressed in Chapter V. It presents an example of an AFTI-F16 digital controller deployed on a computer platform equipped with a novel fault tolerant mechanism. It is shown that the closed-loop system can be modeled with a HJLS. A detailed model is then introduced using data taken from (Tejada 2002). Finally, mean square stability of the model is analyzed with both the tools presented in Chapter IV and Monte Carlo simulations.

In Chapter VI, a summary of the dissertation is presented. A list of conclusions and open problems for future research is also included.

\section{I.4 A WORD ON NOTATION}

As customary, $\mathbb{R}$ and $\mathbb{R}^{+}$denote, respectively, the set of real numbers and the set of non-negative real numbers. $\mathbb{R}^{n}$ denotes the space of $n$-dimensional column vectors with real components, while $\mathbb{R}^{n \times n}$ denotes the set of $n \times n$-dimensional matrices with real components. Likewise, $\mathbb{Z}$ and $\mathbb{Z}^{+}$represent, respectively, the set of integers and non-negative integers. For any $A \in \mathbb{R}^{n \times n}, \bar{\sigma}(A), \underline{\sigma}(A), \lambda_{\max }(A), \lambda_{\min }(A)$, and $\rho(A)$ represent, respectively, $A$ 's maximum and minimum singular value, its maximum and minimum eigenvalue in modulus, and its spectral radius. Throughout the text, $E_{\ell}$ and $I_{n}$ represent, respectively, an $\ell \times \ell$ matrix of ones and an $n \times n$ identity matrix.

Countable sets are denoted using curly brackets. For instance, $\left\{\alpha_{1}, \ldots, \alpha_{\ell}\right\}, \alpha_{i} \in$ $\mathbb{R}$, represents a finite set of $\ell$ real numbers. Finite sets that require a specific name will be denoted by $\Sigma_{\diamond}$ and their cardinality by $\ell_{\diamond}=\left|\Sigma_{0}\right|$, where $\diamond$ represents any appropriate label. However, finite sets of the specific form $\{0,1, \ldots, \ell-1\}$ will be denoted by $\mathcal{I}_{\ell}$. As usual, $2^{\Sigma_{\diamond}}$ (or $2^{\mathcal{I}_{\ell}}$ ) denotes the power set of $\Sigma_{\diamond}\left(\right.$ or $\mathcal{I}_{\ell}$ ). If $\Sigma_{\diamond}$ 
represents a set of symbols, then $\Sigma_{\diamond}^{\infty} \triangleq\left\{\left(\diamond_{0}, \diamond_{1} \ldots\right): \diamond_{i} \in \Sigma_{\diamond}\right\}$ represents the set of all the infinite sequences formed by elements of $\Sigma_{\diamond}$. Likewise, $\mathcal{I}_{\ell}^{\infty} \triangleq\left\{\left(l_{0}, l_{1}, \ldots\right): l_{i} \in \mathcal{I}_{\ell}\right\}$ represents the set of all the infinite sequences formed by elements of $\mathcal{I}_{\ell}$. Discretetime signals (or functions) are considered countable sets. They will be denoted, for example, by $\{\theta(k), k \geq 0\}$ or $\{N(k), k \geq 0\}$. If, for instance, $N(k) \in \mathcal{I}_{\ell}$ for all $k \geq 0$, then it follows that $\{N(k), k \geq 0\} \in \mathcal{I}_{\ell}^{\infty}$. Similarly, if $\theta(k) \in \Sigma_{\circ}$ for all $k \geq 0$, then $\{\theta(k), k \geq 0\} \in \Sigma_{\diamond}^{\infty}$. Uncountable sets are denoted by $\Omega$ and $\Phi$. Their elements are denoted, respectively, by $\omega$ and $\phi$ and their various subsets by $\{\omega: \cdots\}$ and $\{\phi: \cdots\}$ (or, if needed to add clarity, by $\{\omega \in \Omega: \cdots\}$ and $\{\phi \in \Phi: \cdots\}$ ).

This document makes intensive use of several measure theoretic concepts (Borel and measurable functions; random variables, vectors, and processes; etc.), which are reviewed in Appendix A. It is assumed throughout the dissertation that every random variable, random vector, or stochastic process is defined over the same underlying probability space, $(\Omega, \mathscr{F}, \operatorname{Pr})$. Random variables, vectors, and elements are denoted by lower case bold letters $\boldsymbol{x}$ or $\boldsymbol{y}$. The notation, $\boldsymbol{x}(\omega)$ or $\boldsymbol{y}(\omega)$, will be used sparingly to emphasize, when needed, the dependence of $\boldsymbol{x}$ or $\boldsymbol{y}$ on $\omega \in \Omega . \mu^{x}$ and $\mu^{y}$ will be used to denote the probability distributions (also called probability measures) of $\boldsymbol{x}$ and $\boldsymbol{y}$. Stochastic processes are denoted by $\{\boldsymbol{\theta}(k), k \geq 0\},\{\boldsymbol{N}(k), k \geq 0\}$ (or $\{\boldsymbol{\theta}(k, \omega), k \geq 0\},\{\boldsymbol{N}(k, \omega), k \geq 0\}$ ). This implies that, at every time $k \geq 0, \boldsymbol{\theta}(k)$ and $N(k)$ are random variables with distributions $\mu_{k}^{\theta}$ and $\mu_{k}^{N}$, respectively. It follows from the notation introduced above that $\{\theta(k, \omega), k \geq 0\}$ and $\{N(k, \omega), k \geq 0\}$ for a fixed $\omega \in \Omega$ represent, respectively, specific sample paths of the processes $\{\boldsymbol{\theta}(k), k \geq$ $0\}$ and $\{N(k), \geq 0\}$. 
The cross product of two random variables (or vectors), $\boldsymbol{x}$ and $\boldsymbol{y}$, is denoted by $(\boldsymbol{x}, \boldsymbol{y})$, and its distribution is denoted by $\mu^{x, y}$. Note that if $\boldsymbol{x}$ and $\boldsymbol{y}$ are independent, then $\mu^{x, y}=\mu^{x} \mu^{y}$. Similarly, the cross product of two stochastic processes, $\{\boldsymbol{\theta}(k), k \geq$ $0\}$ and $\{\boldsymbol{N}(k), k \geq 0\}$, is denoted by $\{(\boldsymbol{\theta}(k), \boldsymbol{N}(k)), k \geq 0\}$, where $(\boldsymbol{\theta}(k), \boldsymbol{N}(k))$ has distribution $\mu_{k}^{\theta, N}$. As usual, for any random variable or vector $\boldsymbol{x}, \sigma(\boldsymbol{x})$ denotes the smallest $\sigma$-algebra with respect to which $\boldsymbol{x}$ is measurable. Finally, $\mathbf{E}\{\cdot\}$ will be used to denote the expectation operator. 


\section{CHAPTER II}

\section{THE HYBRID JUMP LINEAR SYSTEM MODEL}

\section{II.1 INTRODUCTION}

This chapter describes the hybrid jump linear system model and its various subclasses. The presentation is arranged in two sections. Section II.2 introduces the basic concepts of finite state machines, which are used later to represent the decision making supervisors. Next, two types of HJLS's are introduced: general and canonical HJLS's. General HJLS's have a structure similar to DSHA (Bemporad \& Di Cairano 2005) and are well suited to model general hybrid systems. Canonical HJLS's are introduced to simplify the derivation of the stability conditions introduced in Chapter IV. They are general HJLS's in which the Supervisors are represented by Moore FSM's with identity output maps. At the end of Section II.2, it is argued that given a HJLS with an embedded Moore FSM, it is possible to construct a canonical HJLS with equivalent stability properties (the complete proof of this proposition appears in Appendix C). Consequently, the stability of any HJLS with an embedded Moore FSM can be studied with the results presented in Chapter IV. Section II.3 introduces two more subtypes of general HJLS's: HJLS with performance supervision and HJLS with performance map (the latter is a particular subcase of the former that is specially useful in applications). 


\section{II.2 THE HYBRID JUMP LINEAR SYSTEM MODEL}

As described in the Chapter I, hybrid jump linear systems are composed of a finite state machine, an analog-to-symbol $(A / S)$ map, and a jump linear closed-loop system. The first two components are used to model the behavior of the high level supervisor and its interaction with the closed-loop system, while the latter describes the dynamics of the compensated "plant". Before introducing a detailed description of the supervisor, it is convenient to summarize the basic concepts related to finite state machines.

\section{II.2.1 Finite State Machines}

The formal definition of a FSM follows.

Definition II.2.1 $A$

finite

state

machine

$i s$

a six-tuple, $\mathrm{M}=\left(\Sigma_{N}, \Sigma_{S}, \Sigma_{O}, \delta, \varpi, s_{0}\right)$, where $\Sigma_{N}$ is a finite set of input symbols, $\Sigma_{S}$ is a finite set of states, $\Sigma_{O}$ is a finite set of output symbols, $\delta: \Sigma_{S} \times \Sigma_{N} \mapsto \Sigma_{S}$ is the state transition map, $\varpi: \Sigma_{S} \times \Sigma_{N} \mapsto \Sigma_{O}$ (Mealy FSM) or $\varpi: \Sigma_{S} \mapsto \Sigma_{O}$ (Moore FSM) is the output map, and $s_{0} \in \Sigma_{O}$ is the initial state.

From an input-output perspective, a finite state machine is a device that converts (or maps) infinite input sequences of symbols of the form $\{N(k), k \geq 0\}, N(k) \in \Sigma_{N}$, into infinite output sequences of symbols of the form $\{\theta(k), k \geq 0\}, \theta(k) \in \Sigma_{O}$. The dynamics of the conversion process are given by the state transition map, $\delta$, and the 
output map, $\varpi$, as follows

$$
\begin{aligned}
z(k+1) & =\delta(z(k), N(k)), \quad z(0)=s_{0} \\
\theta(k) & =\varpi(z(k), N(k)) \quad(\text { or } \theta(k)=\varpi(z(k))),
\end{aligned}
$$

where $z(k) \in \Sigma_{S}$ is the FSM state at time $k$ and $\{z(k), k \geq 0\}$ represents the internal state sequence of the FSM. Clearly, a FSM is a dynamical system evolving according to (1). The FSM's dynamics can be expressed in a form more suitable for analysis. To this end, without loss of generality, consider $\Sigma_{S} \triangleq\left\{s_{1}, \ldots, s_{\ell_{S}}\right\}$ to be the set of states of a FSM and define the set of elementary vectors in $\mathbb{R}^{\ell_{S}}, \hat{\Sigma}_{S} \triangleq\left\{e_{1}, \ldots, e_{\ell_{S}}\right\}$, where $\ell_{S}=\left|\Sigma_{S}\right|$ and $e_{i}=\left[\begin{array}{llllllll}0 & \cdots & 0 & 1 & 0 & \cdots & 0\end{array}\right]^{\mathrm{T}}$ with a 1 in the $i$-th entry. Next, define the collection of transition matrices $\mathcal{S} \triangleq\left\{S_{\eta}: \eta \in \Sigma_{N}\right\}$, where each matrix $S_{\eta}$ is defined as follows: if $\left[S_{\eta}\right]_{j}, j \in\left\{1, \ldots, \ell_{S}\right\}$, denotes the $j$-th column of $S_{\eta}$ then $\left[S_{\eta}\right]_{j} \triangleq e_{i}$ whenever $\delta\left(s_{j}, \eta\right)=s_{i}$. Finally, define the map $\hat{\varpi}: \hat{\Sigma}_{S} \times \Sigma_{N} \mapsto \Sigma_{O}$ (or $\hat{\varpi}: \hat{\Sigma}_{S} \mapsto \Sigma_{O}$ ) as follows: $\hat{\varpi}\left(e_{i}, \eta\right)=\varpi\left(s_{i}, \eta\right)$ (or $\hat{\varpi}\left(e_{i}\right)=\varpi\left(s_{i}\right)$ ). It is now possible to define the following dynamical equation

$$
\begin{aligned}
\hat{z}(k+1) & =S_{N(k)} \hat{z}(k), \quad \hat{z}(0)=\hat{z}_{0} \\
\hat{\theta}(k) & =\hat{\varpi}(\hat{z}(k), N(k)) \quad(\text { or } \hat{\theta}(k)=\hat{\varpi}(\hat{z}(k))),
\end{aligned}
$$

where $\hat{z}_{0} \triangleq e_{i}$ whenever $s_{0}=s_{i}$. Clearly, from an input-output perspective, equations (1) and (2) describe the dynamics of the same FSM because, for every $k \geq 0, \hat{z}(k)=e_{i}$ if and only if $z(k)=s_{i}$ and $\hat{\theta}(k)=\theta(k)$. This suggests the following alternative definition of a FSM.

Definition II.2.2 (Alternative Definition) A finite state machine is a sixtuple, $\mathrm{M}=\left(\Sigma_{N}, \hat{\Sigma}_{S}, \Sigma_{O}, \mathcal{S}, \hat{\varpi}, \hat{z}_{0}\right)$, where $\Sigma_{N}$ is a finite set of input symbols, $\hat{\Sigma}_{S}=$ 
$\left\{e_{1}, \ldots, e_{\ell_{S}}\right\}$ is a finite set of states, $\Sigma_{O}$ is a finite set of output symbols, $\mathcal{S}$ is the set of transition matrices, $\hat{\varpi}: \hat{\Sigma}_{S} \times \Sigma_{N} \mapsto \Sigma_{O}$ (Mealy FSM) or $\hat{\varpi}: \hat{\Sigma}_{S} \mapsto \Sigma_{O}$ (Moore FSM) is the output map, and $z_{0} \in \hat{\Sigma}_{O}$ is the initial state.

It is clear from equation (2) that a FSM behaves as a jump linear system. This important fact will be used to develop the stability theory for hybrid jump linear systems presented in Chapters III and IV. In the rest of this dissertation, all the FSM's will be defined using Definition II.2.2. To simplify the notation, the hat $\left({ }^{\wedge}\right)$ notation will be dropped. That is, equation (2) will be simply written as

$$
\begin{aligned}
z(k+1) & =S_{N(k)} z(k), \quad z(0)=z_{0} \\
\theta(k) & =\varpi(z(k), N(k)) \quad(\text { or } \theta(k)=\varpi(z(k))),
\end{aligned}
$$

A description of the general HJLS model is provided in the following three subsections.

\section{II.2.2 The Supervisor}

As shown in Figure 2, the supervisor is composed of two subsystems: a finite state machine and an analog-to-symbol $(A / S)$ map. The FSM, $\mathrm{M}_{S p}$, represents the decision making algorithm of the supervisor. $\mathrm{M}_{S p}$ has two inputs, the stochastic processes $\{\boldsymbol{N}(k), k \geq 0\}$ and $\{\boldsymbol{\nu}(k), k \geq 0\}$, and one output, $\{\boldsymbol{\theta}(k), k \geq 0\}$. Formally, $\mathrm{M}_{S p} \triangleq\left(\mathcal{I}_{\ell_{N}} \times \mathcal{I}_{\ell_{\nu}}, \Sigma_{S}, \mathcal{I}_{\ell_{O}}, \mathcal{S}, \varpi, \mu_{0}^{z}\right)$, where

- $\mathcal{I}_{\ell_{N}}=\left\{0, \ldots, \ell_{N}-1\right\}$ is the finite input set corresponding to $\{N(k), k \geq 0\}$, that is, $\boldsymbol{N}(k) \in \mathcal{I}_{\ell_{N}}$ for every $k \geq 0 .\{\boldsymbol{N}(k), k \geq 0\}$ is assumed to be a discretetime, homogeneous Markov chain with transition probability matrix $\Pi_{N}=\left[p_{i, j}^{N}\right]$ and initial distribution $\mu_{0}^{N}$. 
- $\mathcal{I}_{\ell_{\nu}}=\left\{0, \ldots, \ell_{\nu}-1\right\}$ is the finite input set corresponding to $\{\boldsymbol{\nu}(k), k \geq 0\}$, that is, $\boldsymbol{\nu}(k) \in \mathcal{I}_{\ell_{\nu}}$ for every $k \geq 0 .\{\boldsymbol{\nu}(k), k \geq 0\}$ is the output of the analog-tosymbol $(A / S)$ map.

- $\Sigma_{S}=\left\{e_{1}, e_{2}, \ldots, e_{\ell_{S}}\right\}$, the set of elementary vectors in $\mathbb{R}^{\ell_{S}}$, is the finite set of states, that is, $\boldsymbol{z}(k) \in \Sigma_{S}$ for every $k \geq 0 .\{\boldsymbol{z}(k), k \geq 0\}$ is the state process of $\mathrm{M}_{S_{\mathrm{p}}}$

- $\mathcal{I}_{\ell_{O}}=\left\{0, \ldots, \ell_{O}-1\right\}$ is the finite set of output symbols, that is, $\boldsymbol{\theta}(k) \in \mathcal{I}_{\ell_{O}}$ for every $k \geq 0 .\{\boldsymbol{\theta}(k), k \geq 0\}$ is the output process of $\mathrm{M}_{S_{p}}$.

- $\mathcal{S} \triangleq\left\{S_{\eta, \nu}, \eta \in \mathcal{I}_{\ell_{N}}, \nu \in \mathcal{I}_{\ell_{\nu}}\right\}$ is the finite set of state transition matrices. Therefore, $\{\boldsymbol{z}(k), k \geq 0\}$ evolves according to

$$
\boldsymbol{z}(k+1)=S_{\boldsymbol{N}(k), \boldsymbol{\nu}(k)} \boldsymbol{z}(k), \quad \boldsymbol{z}(0)=\boldsymbol{z}_{0}
$$

Note that the initial state of $\mathrm{M}_{S p}, z_{0}$, is assumed to be random, with initial distribution $\mu_{0}^{z}$.

- $\varpi$ is the output map, which is given by

$$
\begin{aligned}
\varpi: \quad \mathcal{I}_{\ell_{N}} \times \mathcal{I}_{\ell_{\nu}} \times \Sigma_{S} & \rightarrow \mathcal{I}_{\ell_{O}} \\
(\boldsymbol{N}(k), \boldsymbol{\nu}(k), \boldsymbol{z}(k)) & \mapsto \boldsymbol{\theta}(k)=\varpi(\boldsymbol{N}(k), \boldsymbol{\nu}(k), \boldsymbol{z}(k)) .
\end{aligned}
$$

As shown in Figure 2, the second input, $\nu(k)$, is the output of the $A / S$ map, which is represented with a quantization function, $\psi: \mathbb{R}^{n} \rightarrow \mathcal{I}_{\ell_{\nu}}$. It partitions the jump linear closed-loop system's state space, $\mathbb{R}^{n}$, into $\ell_{\nu}$ mutually exclusive Borelmeasurable subsets $\left\{R_{i}, i \in \mathcal{I}_{\ell_{\nu}}\right\} .^{1}$ That is,

$$
\psi(x)=\sum_{i=0}^{\ell_{\nu}-1} i 1_{\left\{x \in R_{i}\right\}}, \quad \bigcup_{i \in \mathcal{I}_{\ell_{\nu}}} R_{i}=\mathbb{R}^{n},
$$

\footnotetext{
${ }^{1}$ Each $R_{i}$ is required to be Borel-measurable to ensure that the expression $\operatorname{Pr}\left\{x(k) \in R_{i}\right\}$ is well defined. This will be important in Chapter IV.
} 


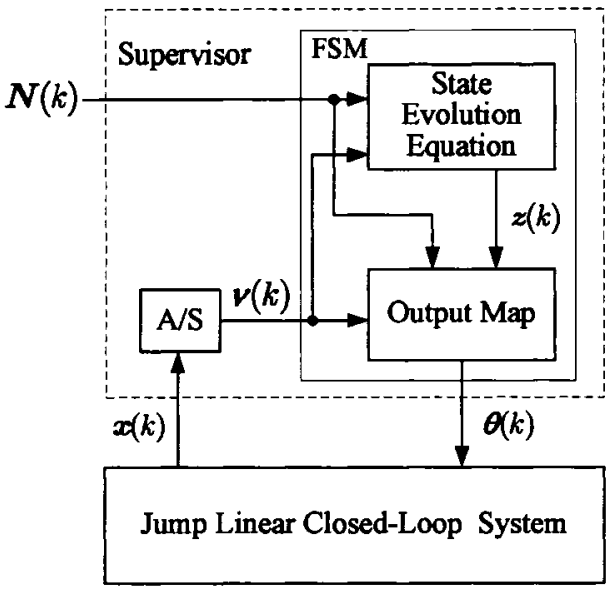

FIG. 4: A general HJLS.

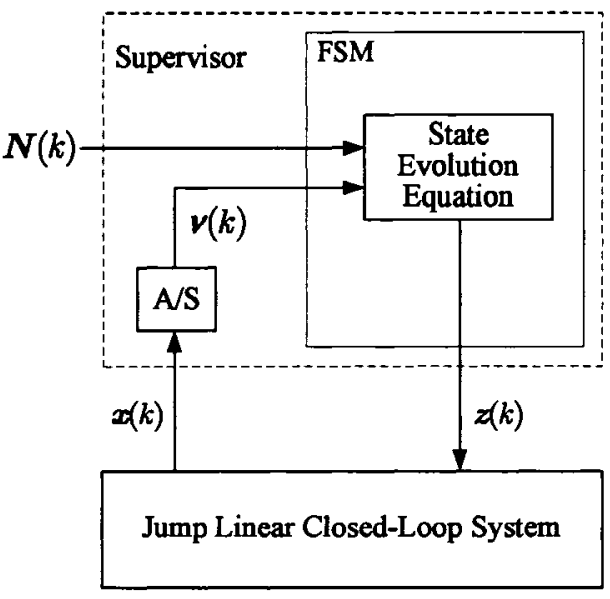

FIG. 5: A canonical HJLS

where $R_{i} \in \mathscr{B}\left(\mathbb{R}^{n}\right)$, for every $i \in \mathcal{I}_{\ell_{\nu}}$, and $\mathbf{1}_{\left\{x \in R_{i}\right\}}$ (or $\mathbf{1}_{R_{i}}(x)$ ) denotes the indicator function, which equals 1 if $x \in R_{i}$ and 0 otherwise.

\section{II.2.3 The Jump Linear Closed-Loop System}

The dynamics of the closed-loop system in Figure 2 are represented by a jump linear system with state vector $\boldsymbol{x}(k) \in \mathbb{R}^{n}$, whose modes are selected by the FSM's output sequence according to

$$
\boldsymbol{x}(k+1)=A_{\boldsymbol{\theta}(k)} \boldsymbol{x}(k), \quad \boldsymbol{x}(0)=\boldsymbol{x}_{0},
$$

where $A_{\boldsymbol{\theta}(k)}=A_{i} \in \mathbb{R}^{n \times n}$, whenever $\boldsymbol{\theta}(k)=i \in \mathcal{I}_{\ell_{O}}$. The closed-loop system's initial vector, $\boldsymbol{x}_{0}$, is assumed to be an integrable random vector independent of $\boldsymbol{z}_{0}$ and $\{N(k), k \geq 0\}$ with distribution $\mu_{0}^{x}$. 


\section{II.2.4 Formal Definition of a HJLS}

The (internal) state of a hybrid jump linear system is given by $\left[\boldsymbol{x}^{\mathrm{T}}(k) \boldsymbol{z}^{\mathrm{T}}(k)\right]^{\mathrm{T}}$, which yields the following jump system representation

$$
\left[\begin{array}{c}
\boldsymbol{x}(k+1) \\
\boldsymbol{z}(k+1)
\end{array}\right]=\left[\begin{array}{cc}
A_{\boldsymbol{\theta}(k)} & 0 \\
0 & S_{\boldsymbol{N}(k), \boldsymbol{\nu}(k)}
\end{array}\right]\left[\begin{array}{l}
\boldsymbol{x}(k) \\
\boldsymbol{z}(k)
\end{array}\right] .
$$

This is not a jump linear representation. This is made evident by noting that $A_{\boldsymbol{\theta}(k)}=A_{\varpi(\boldsymbol{N}(k), \boldsymbol{\nu}(k), \boldsymbol{z}(k))}$ and $S_{\boldsymbol{N}(k), \boldsymbol{\nu}(k)}=S_{(\boldsymbol{N}(k), \psi(\boldsymbol{x}(k)))}$ (see (4) and the $A / S$ map definition). In the sequel, $\boldsymbol{y}(k)$ will be used to represent the state of a HJLS, that is, $\boldsymbol{y}(k) \triangleq\left[\boldsymbol{x}^{\mathrm{T}}(k) \boldsymbol{z}^{\mathrm{T}}(k)\right]^{\mathrm{T}}$. This simplifies the HJLS formal definition that follows.

Definition II.2.3 The system in Figure 4 described by (3)-(6) is called a hybrid jump linear system. Its state evolution is given by

$$
\boldsymbol{y}(k+1)=\left[\begin{array}{cc}
A_{\boldsymbol{\theta}(k)} & 0 \\
0 & S_{\boldsymbol{N}(k), \nu(k)}
\end{array}\right] \boldsymbol{y}(k), \quad \boldsymbol{y}(0)=\boldsymbol{y}_{0}
$$

where $\boldsymbol{y}(k) \in Y \triangleq \mathbb{R}^{n} \times \Sigma_{S}$, and the initial condition, $\boldsymbol{y}_{0}=\left[\begin{array}{ll}\boldsymbol{x}_{0}^{\mathrm{T}} & \boldsymbol{z}_{0}^{\mathrm{T}}\end{array}\right]^{\mathbf{T}}$, has distribution $\mu_{0}^{y}=\mu_{0}^{x} \mu_{0}^{z}$

The HJLS paradigm shown in Figure 4 is well suited to model a large class of state-dependent supervisory applications. As mentioned in the introduction, the stability of the general HJLS introduced here will be analyzed in Chapters III and IV. This analysis will be significantly simplified for a special subclass of HJLS's to be introduced next.

\section{II.2.5 Canonical Hybrid Jump Linear Systems}

Consider a HJLS with a Moore FSM equipped with an identity output map as shown in Figure 5. For this system, the FSM's state evolution equation and output 
map are given by

$$
\begin{aligned}
\boldsymbol{z}(k+1) & =S_{\boldsymbol{N}(k), \boldsymbol{\nu}(k)} \boldsymbol{z}(k) \\
\boldsymbol{\theta}(k) & =\varpi(\boldsymbol{z}(k))=\boldsymbol{z}(k) .
\end{aligned}
$$

Consequently, the closed-loop system's dynamics are given by

$$
\boldsymbol{x}(k+1)=A_{\boldsymbol{z}(k)} \boldsymbol{x}(k), \quad \boldsymbol{x}(0)=\boldsymbol{x}_{0},
$$

where $A_{\boldsymbol{z}(k)}=A_{i} \in \mathbb{R}^{n \times n}$, whenever $\boldsymbol{z}(k)=e_{i} \in \Sigma_{S}$.

Remark II.2.1 Observe that in a canonical HJLS, the output symbol set is given by $\Sigma_{S}$ (instead of $\left.\mathcal{I}_{\ell_{0}}\right)$. Consequently, the matrices $A_{\boldsymbol{z}(k)}$ in $(10)$ take values from the set $\left\{A_{1}, \ldots, A_{\ell_{S}}\right\}$.

Definition II.2.4 The system in Figure 5 described by (5), (9), and (10) is called a canonical hybrid jump linear system with state evolution given by

$$
\boldsymbol{y}(k+1)=\left[\begin{array}{cc}
A_{\boldsymbol{x}(k)} & 0 \\
0 & S_{N(k), \nu(k)}
\end{array}\right] \boldsymbol{y}(k), \quad \boldsymbol{y}(0)=\boldsymbol{y}_{0}
$$

The focus on canonical HJLS is justified by the fact that for any general HJLS with an embedded Moore FSM, it is possible to construct a canonical HJLS with equivalent stability properties. The proof of this claim makes use of concepts developed in Chapter IV and can be found in Appendix C. To understand the construction process for a canonical HJLS, first consider the following HJLS with embedded Moore FSM:

$$
\begin{aligned}
\boldsymbol{x}(k+1) & =A_{\varpi(\boldsymbol{z}(k))} \boldsymbol{x}(k), \quad \boldsymbol{x}(0)=\boldsymbol{x}_{0} \\
\boldsymbol{z}(k+1) & =S_{\boldsymbol{N}(k), \psi(\boldsymbol{x}(k))} \boldsymbol{z}(k) \\
\psi(x) & =\sum_{i=0}^{\ell_{\nu}-1} i \mathbf{1}_{\left\{x \in R_{i}\right\}}, \quad \bigcup_{i \in \mathcal{I}_{\ell_{\nu}}} R_{i}=\mathbb{R}^{n},
\end{aligned}
$$


where $A_{\varpi(z(k))}=A_{l}$, whenever $\varpi(z(k))=l \in \mathcal{I}_{\ell_{0}}$. Assume the supervisor's FSM is given by $\mathrm{M}_{S_{p}}=\left(\mathcal{I}_{\ell_{N}} \times \mathcal{I}_{\ell_{\nu}}, \Sigma_{S}, \mathcal{I}_{\ell_{0}}, \mathcal{S}, \varpi, \mu_{0}^{z}\right)$. Next, consider the FSM, $\tilde{\mathrm{M}}_{S_{p}}$, given by $\tilde{\mathrm{M}}_{S_{p}}=\left(\mathcal{I}_{\ell_{N}} \times \mathcal{I}_{\ell_{\nu}}, \Sigma_{S}, \Sigma_{S}, \mathcal{S}, \tilde{\varpi}, \mu_{0}^{z}\right)$, where $\tilde{\varpi}$ is an identity output map, i.e., $\tilde{\varpi}(e)=e$ for all $e \in \Sigma_{S}$. Finally, embedding $\tilde{\mathrm{M}}$ in a HJLS structure yields the desired equivalent canonical HJLS

$$
\begin{aligned}
\tilde{\boldsymbol{x}}(k+1) & =\tilde{A}_{\tilde{\boldsymbol{z}}(k)} \tilde{\boldsymbol{x}}(k), \quad \tilde{\boldsymbol{x}}(0)=\boldsymbol{x}_{0} \\
\tilde{\boldsymbol{z}}(k+1) & =S_{\boldsymbol{N}(k), \psi(\overline{\boldsymbol{x}}(k))} \boldsymbol{z}(k) \\
\psi(x) & =\sum_{i=0}^{\ell_{\nu}-1} i \mathbf{1}_{\left\{x \in R_{i}\right\}}, \quad \bigcup_{i \in \mathcal{I}_{\ell_{\nu}}} R_{i}=\mathbb{R}^{n},
\end{aligned}
$$

where $\tilde{A}_{\tilde{z}(k)}=A_{i}$, whenever $\varpi(\tilde{\boldsymbol{z}}(k))=i \in \mathcal{I}_{\ell_{O}}$.

The jump linear closed-loop system in (12) may have more modes than the original system in (11). It is easy to see, however, that if both HJLS's are initialized with the same initial state, $y_{0}$, then $\boldsymbol{x}(k)=\tilde{\boldsymbol{x}}(k)$ for all $k \geq 0$. This condition, which is called A-equivalency (Patilkulkarni et al. 2004), implies that the stability characteristics of the HJLS's in (11) and (12) are identical. A more complete treatment of this concept is given in Appendix C.

\section{II.3 HYBRID JUMP LINEAR SYSTEMS WITH PERFORMANCE SUPERVISION}

This section introduces two additional subtypes of HJLS with structure imposed on the $A / S$ map and the FSM that are typical in applications (see Chapter III). The stability analysis of these special HJLS's can be carried out with a simpler methodology than that required to analyze the stability of canonical HJLS. Moreover, by 


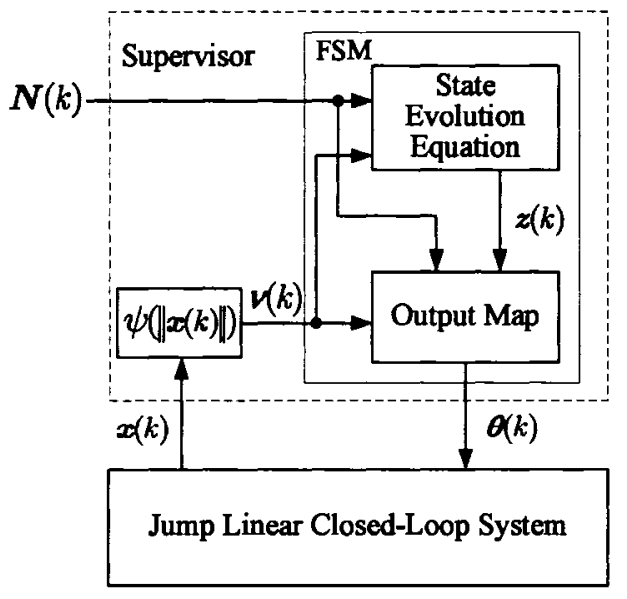

FIG. 6: A HJLS with performance supervision.

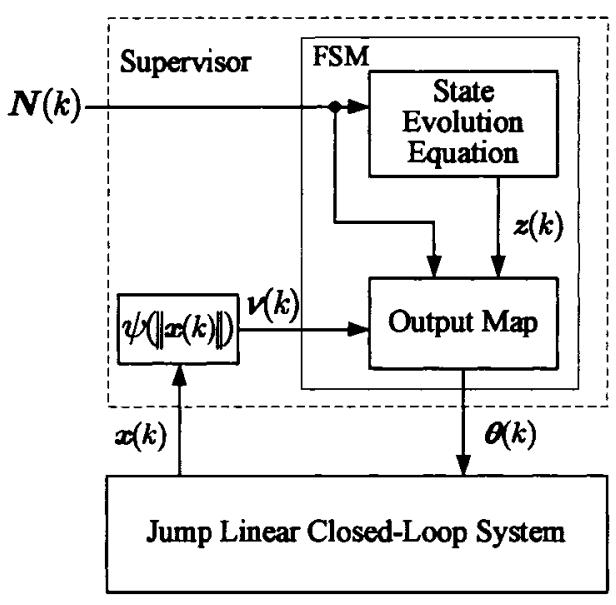

FIG. 7: A HJLS with performance map.

analyzing these simpler systems first, one can obtain useful insight on the behavior of general HJLS's.

\section{II.3.1 Hybrid Jump Linear Systems with Performance Supervision}

It is assumed here that the $A / S$ map provides a measure of the jump linear closedloop system performance to the supervising algorithm. The performance metric of interest is a quantization of the value of the norm of the closed-loop system's state vector, $\boldsymbol{x}(k)$. Specifically, for a finite set of $\ell_{\nu}+1$ positive constants satisfying $0=$ $\alpha_{0}<\alpha_{1}<\cdots<\alpha_{\ell_{\nu}-1}<\alpha_{\ell_{\nu}}=\infty$, the $A / S$ map is given by

$$
\boldsymbol{\nu}(k)=\psi(\|\boldsymbol{x}(k)\|)=\sum_{i=0}^{\ell_{\nu}-1} i \mathbf{1}_{\left\{\alpha_{i} \leq\|\boldsymbol{x}(k)\|<\alpha_{i+1}\right\}},
$$

where $\mathbf{1}_{\left\{\alpha_{i} \leq\|x(k)\|<\alpha_{i+1}\right\}}=1$ whenever $\alpha_{i} \leq\|\boldsymbol{x}(k)\|<\alpha_{i+1}$ and zero otherwise. Note that the $A / S$ map quantizes the range of $\|x(k)\|$ into $\ell_{\nu}$ levels, which is expected since $\nu(k) \in \mathcal{I}_{\ell_{\nu}}$. Also note that the boundaries between the quantization regions are hyperspheres in $\mathbb{R}^{n}$. Formally, this subtype of HJLS is defined as follows. 
Definition II.3.1 A hybrid jump linear system as described by (3), (4), (6), and (13) is called a Hybrid Jump Linear System with Performance Supervision. Its state evolution is given by (8).

\section{II.3.2 Hybrid Jump Linear Systems with Performance Map}

In addition to restricting the $A / S$ map as in (13), this subclass of HJLS's assumes that the supervisor performs its decision process in two steps. In the first step, the supervisor uses the information from the external input $N(k)$ and its current state, $\boldsymbol{z}(k)$, to determine the 'best' subclass of modes to apply to the closed-loop system at the next sample instant. During the second step, the supervisor uses the performance information provided by the $A / S$ map to make the final mode selection from the subclass of modes selected in the first step. Thus, the FSM's state evolution equation depends effectively only on $\boldsymbol{N}(k)$, while its output map depends on both $\boldsymbol{N}(k)$ and $\boldsymbol{\nu}(k)$. Since the output map performs the final mode selection based on performance information, it will be called the performance map. This map has a very special structure, which makes it amenable for stability analysis, as will be evident in Chapter III. Note that under the considerations described above, the FSM's state evolution equation (3) reduces to

$$
\boldsymbol{z}(k+1)=S_{\boldsymbol{N}(k)} \boldsymbol{z}(k)
$$

The FSM's output, $\{\boldsymbol{\theta}(k), k \geq 0\}$, is generated by the specific output (performance) map given next. It is precisely this representation which simplifies the stability analysis. Observe

$$
\boldsymbol{\theta}(k)=\varpi(\boldsymbol{N}(k), \boldsymbol{\nu}(k), \boldsymbol{z}(k))
$$




$$
\begin{aligned}
& =\left(\ell_{N} \cdot \ell_{S}\right) \boldsymbol{\nu}(k)+\ell_{N}\left[0,1, \ldots, \ell_{S}-1\right] \boldsymbol{z}(k)+\boldsymbol{N}(k) \\
& =\left(\ell_{N} \cdot \ell_{S}\right) \boldsymbol{\nu}(k)+\ell_{N} \tilde{\boldsymbol{z}}(k)+\boldsymbol{N}(k) \\
& =\left(\ell_{N} \cdot \ell_{S}\right) \boldsymbol{\nu}(k)+\boldsymbol{\varphi}(k),
\end{aligned}
$$

where $\boldsymbol{\varphi}(k) \triangleq \ell_{N} \hat{\boldsymbol{z}}(k)+\boldsymbol{N}(k)$ and $\tilde{\boldsymbol{z}}(k) \triangleq\left[0,1, \ldots, \ell_{S}-1\right] \boldsymbol{z}(k)$. Thus, if $\boldsymbol{z}(k)=e_{j}$ then $\hat{z}(k)=j-1 \in \mathcal{I}_{\ell_{S}}$. Clearly, $\tilde{z}(k)$ is isomorphic to $\boldsymbol{z}(k)$. Also, observe that $\varphi(k)=\Psi(N(k), \tilde{z}(k))$, where $\Psi: \mathcal{I}_{\ell_{N}} \times \mathcal{I}_{\ell_{S}} \rightarrow \mathcal{I}_{\ell_{N} \cdot \ell_{S}}:(e, d) \mapsto f=\ell_{N} \cdot d+e$ is a bijective map. Thus, $\varphi(k)$ is isomorphic to the random process $(\boldsymbol{N}(k), \boldsymbol{z}(k))$, which is known to be a Markov chain (see Chapter IV Section IV.3 and (Patilkulkarni et al. 2004)). Consequently, $\boldsymbol{\varphi}(k)$ is also a Markov chain. Notice, however, that $\boldsymbol{\theta}(k)$ may not be a Markov chain since $\boldsymbol{\nu}(k)$ may not be memoryless. The formal definition of this subclass of HJLS's follows.

Definition II.3.2 A hybrid jump linear system as described by (6) and (13)-(15) is called a Hybrid Jump Linear System with Performance Map. Its state evolution is given by (8).

Figures 6 and 7 show block diagrams for these subtypes of HJLS.

\section{II.4 CHAPTER SUMMARY AND CONCLUSIONS}

This chapter presented the general hybrid jump linear system model. It also summarized the basic properties of finite state machines, which are used to model the HJLS's supervisor and provided a description of three specific subclasses of HJLS's: HJLS's with performance supervision, HJLS's with performance map, and canonical 
HJLS's. In addition, this chapter introduced two important concepts to be employed in the stability analysis presented in the subsequent chapters:

- General HJLS can be subdivided into two subclasses: those with embedded Moore FSM's and those with embedded Mealy FSM's. A HJLS of the first class can always be restated as a canonical HJLS with equivalent stability properties. The stability of the latter, however, is simpler to analyze. This motivates the focus in Chapter IV on canonical HJLS.

- A FSM is usually specified by means of a state transition diagram or table, which is an alternative representation of its state transition map, $\delta$. However, it is clear from the discussion in Subsection II.2.1 that these representations can be easily transformed into the formalism of Definition II.2.2. As a consequence, one can design a HJLS's supervisor using state transition diagrams, the usual tool for algorithm design. This idea will be used in Chapter V. 


\section{CHAPTER III}

\section{STABILITY ANALYSIS OF HYBRID JUMP LINEAR SYSTEMS}

\section{III.1 INTRODUCTION}

This chapter presents a framework and basic stability analysis theory for hybrid jump linear systems. It will be shown in Section III.2 that the essential component of a HJLS, for stability analysis, is its embedded jump linear closed-loop system. This JLS has no external "reference" or "tracking" inputs. The effect of the HJLS's input process, $\{N(k), k \geq 0\}$, is reflected only through the mode-switching process, $\{\boldsymbol{\theta}(k), k \geq 0\}$. From this perspective, both the jump linear closed-loop system and the HJLS are autonomous systems. Thus, only zero input stability is considered in this research, and emphasis will be placed on deriving testable stability conditions.

Four goals are pursued in this chapter: to establish an appropriate stability analysis framework for HJLS's, to introduce the relevant stability concepts for these systems, to illustrate different stability analysis techniques that lead to an array of testable stability conditions, and to derive a stability condition for a JLS driven by general, finite-state stochastic processes. The first two goals are addressed in Section III.3 where a stability analysis framework based on the concepts developed by Hou and Michel (Hou \& Michel 2001a) is described. This section also discusses a particular invariant set, $\mathfrak{M}$, for which stability definitions can be written in terms of only the equilibrium point, $x_{e}=0$, of the embedded jump linear closed-loop system. In this 
way, several stability concepts developed for jump linear systems can be extended to HJLS's. Moreover, through this framework, the stability of HJLS can be studied with techniques similar to those used to analyze switched systems and Markov jump linear systems.

The third and fourth goals are achieved in Sections III.3, III.4, and III.5. The work is focused on mean square stability (MSS) for three reasons: First, it is one of the two strongest forms of stochastic convergence (the other one is almost sure stability (Papoulis 1991)). Second, it is well-known that for hybrid systems (particularly for Markov jump linear systems), MSS implies the existence of solutions for optimal linear quadratic regulator design problems (cf. Costa et al. 2005). Finally, as explained in Chapter I, MSS is also used in performance analysis of HJLS's (Zhang et al. 2005). Several testable sufficient mean square stability conditions will be derived based on different techniques. Section III.3 views HJLS's as switched systems. It presents two new testable conditions, one necessary and one sufficient, for the asymptotic stability of linear autonomous switched systems under arbitrary switching. The latter condition is also valid for HJLS's. Section III.4 studies the stability of HJLS's using singular value techniques. It derives several mean square stability conditions based on restrictions imposed on the singular values of the state matrices of a embedded jump linear system. Section III.5 applies a lifting technique similar to the one used by (Costa \& Fragoso 1993) to study Markov jump linear systems. Two new sufficient MSS conditions are presented in this context. The first MSS condition applies only to HJLS's with performance map. The second one applies to any autonomous jump linear system. Its application requires that certain statistics about its switching 


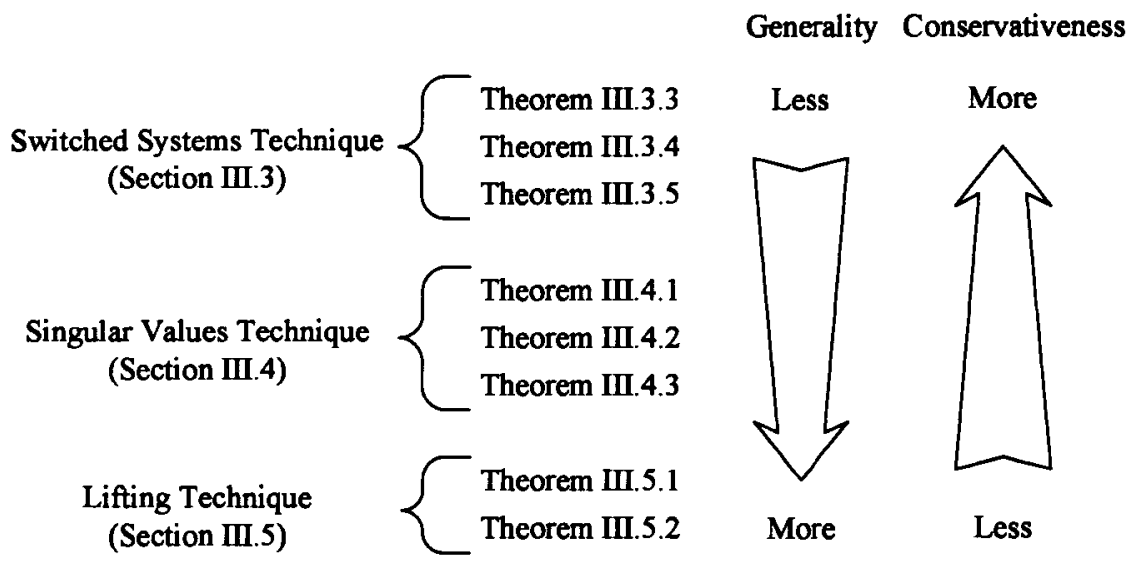

FIG. 8: The organization of the main results in this chapter.

process, $\{\boldsymbol{\theta}(k), k \geq 0\}$, be estimated. This last result, which is presented in Theorem III.5.2, is the main result of this chapter.

It is important to note that the stability conditions in this chapter have been (loosely) arranged in increasing order of generality and decreasing order of conservativeness (see Figure 8). Section III.6, at the end of the chapter, illustrates the main results through several simple numerical examples and Monte Carlo simulations.

\section{III.2 STABILITY ANALYSIS FRAMEWORK}

Intuitively, since the jump linear closed-loop system is autonomous, one would expect that a HJLS would remain in equilibrium whenever $x(k)=0$, regardless of what its supervisor does. Consequently, it makes sense to look for conditions that establish the boundedness and convergence of the continuous subsystems of a hybrid jump linear system (its jump linear system). This idea is not new. It is called Lagrange stability by some authors (cf. Hassibi, Boyd \& How 1999, Ferrari-Trecate, 
Cuzzola \& Morari 2003) and has been used to study the stability of discrete hybrid automata (Torrisi \& Bemporad 2004).

This section will introduce a stability analysis framework that equates the study of the stability of a HJLS with the stability of the equilibrium point, $x_{e}=0$, of the embedded jump linear system, regardless of the state of the embedded finite state machine, $\boldsymbol{z}(k)$. In practice, however, stability is not only a property of the equilibrium point of the jump linear system but also of a subset of the states of the finite state machine. So it is important to study the stability of a subset of $Y=\mathbb{R}^{n} \times \Sigma_{S}$ that includes the equilibrium point of the jump linear system and all or some of the states of the finite state machine. The framework presented next will also make it easier to analyze a sampled-data version of the jump linear system in Figure 2, consisting of a continuous-time plant interfaced via $\mathrm{A} / \mathrm{D}$ and $\mathrm{D} / \mathrm{A}$ converters to a discretetime jump linear controller. The relevant stability definitions will be adapted from (Hou \& Michel 2001a), generalizing some of the standard jump linear system stability definitions to the hybrid jump linear system case.

For stability analysis, the sample solutions (or motions) of interest correspond to the stochastic process $\{\boldsymbol{y}(k), k \geq 0\}$ (see (8)). At each time instant $k, \boldsymbol{y}(k)$ maps samples $\omega \in \Omega$ into $Y$. Thus, a useful metric space for the desired stability analysis is $(Y, d)$, where the metric $d: Y \rightarrow \mathbb{R}^{+}$is defined by

$$
d\left(\left[\begin{array}{l}
x \\
z
\end{array}\right],\left[\begin{array}{c}
\tilde{x} \\
\tilde{z}
\end{array}\right]\right)=\|x-\tilde{x}\|+|z-\tilde{z}|
$$

with $x, \tilde{x} \in \mathbb{R}^{n}, z, \tilde{z} \in \Sigma_{S}$, and $\|\cdot\|$ and $|\cdot|$ denote the Euclidean norm in $\mathbb{R}^{n}$ and the discrete metric, respectively. The distance between $y \in Y$ and a set $M \subset Y$ is defined in the usual manner as $d(y, M)=\inf \{d(y, m): m \in M\}$. 
Definition III.2.1 (Stochastic Motion of a HJLS) Let $(Y, d)$ be the metric space in (16). Consider a HJLS in (8) with initial time $k_{0}=0$ and a fxed initial state $y_{0}=\left[\begin{array}{ll}x_{0}^{\mathrm{T}} & z_{0}^{\mathrm{T}}\end{array}\right]^{\mathrm{T}} \in Y$. Let $\{N(k), k \geq 0\}$ be a Markov chain with initial distribution $\mu_{0}^{N}$ and transition probability matrix $\Pi_{N}$. A stochastic process $\left\{\phi\left(k, \omega, y_{0}, \mu_{0}^{N}\right), k \geq k_{0}\right\}$ is called a stochastic motion of (8) if for each $\omega \in \Omega,\left\{\phi\left(k, \omega, y_{0}, \mu_{0}^{N}\right), k \geq k_{0}\right\}$ is a sample solution of (8) and $\phi\left(0, \omega, y_{0}, \mu_{0}^{N}\right)=y_{0}$ for all $\omega \in \Omega$.

Observe that it is always possible to define a stochastic motion for a hybrid jump linear system. Specifically, fix any $\omega \in \Omega$ and note that under the sample path (or input signal) $\{N(k, \omega), k \geq 0\}$, the dynamics of the hybrid jump linear system in (8) are governed by a set of difference equations. Thus, there always exists a unique trajectory (or solution), $\left\{\phi\left(k, \omega, y_{0}, \mu_{0}^{N}\right), k \geq k_{0}\right\}$, for the HJLS. The collection of these trajectories is exactly the stochastic motion $\left\{\phi\left(k, \omega, y_{0}, \mu_{0}^{N}\right), k \geq k_{0}\right\}$.

Definition III.2.2 (Stochastic Dynamical System) Let $\mathfrak{s}$ be a collection of the stochastic motions corresponding to a subset of fuxed initial states $y_{0} \in Y$ and/or a subset of initial distributions $\mu_{0}^{N}$. This collection together with the set $\mathbb{Z}^{+}$of time values and the metric space $(Y, d)$ is called a stochastic dynamical system and it is represented by $\left\{\mathbb{Z}^{+}, Y, d, \mathfrak{s}\right\}$.

Remark III.2.1 For any given HJLS, the collection of stochastic motions associated with all the valid fixed initial conditions, $y_{0} \in Y$, and all valid initial distributions, $\mu_{0}^{N}$, will be denoted by $\mathfrak{G}$. 


\section{III.2.1 Stability Concepts}

The following definitions and lemma provide the core concepts for analyzing stability of hybrid jump linear systems. In the sequel, $\mathfrak{s} \subset \mathfrak{S}$ denotes a set of stochastic motions of a HJLS. Also, unless otherwise stated, it is assumed that $k_{0}=0$.

Definition III.2.3 (Invariant Set of a HJLS) $A$ set $M \subset Y$ is said to be invariant with respect to $\mathfrak{s}$, or simply $M$ is $\mathfrak{s}$-invariant, if $m \in M$ implies that $\operatorname{Pr}\left\{d\left(\phi\left(k, \omega, m, \mu_{0}^{N}\right), M\right)=0, \forall k \geq 0\right\}=1$, for every stochastic motion $\left\{\phi\left(k, \omega, m, \mu_{o}^{N}\right), k \geq 0\right\}$ in $\mathfrak{s}$.

Definition III.2.4 (Equilibrium Point of a HJLS) $A$ point $y_{e} \in Y$ is called an equilibrium point of $\mathfrak{s}$ if $\left\{y_{e}\right\}$ is $\mathfrak{s}$-invariant.

Definition III.2.5 (Stability in the Second Mean) Consider the stochastic dynamical system $\left\{\mathbb{Z}^{+}, Y, d, \mathfrak{s}\right\}$ defined for a given HJLS. $A$ set $M \subset Y$ is said to be stable in the second mean, if for any $\epsilon>0$ there exists $\delta=\delta(\epsilon)>0$ such that

$$
\mathbf{E}\left\{d^{2}\left(\phi\left(k, \omega, y_{0}, \mu_{0}^{N}\right), M\right)\right\}<\epsilon, \forall k \geq 0
$$

for every stochastic motion $\left\{\phi\left(k, \omega, y_{0}, \mu_{0}^{N}\right), k \geq 0\right\} \in \mathfrak{s}$ where $d\left(y_{0}, M\right)<\delta$.

\section{Definition III.2.6 (Asymptotic Stability in the Second Mean )}

Consider the stochastic dynamical system $\left\{\mathbb{Z}^{+}, Y, d, \mathfrak{s}\right\}$ defined for a given HJLS. A set $M \subset Y$ is said to be asymptotically stable in the second mean, if it is stable in the second mean and if for any $\epsilon>0$ there exists $\delta=\delta(\epsilon)>0$ and $\tau=\tau(\epsilon)$ such that

$$
\mathbf{E}\left\{d^{2}\left(\phi\left(k, \omega, y_{0}, \mu_{0}^{N}\right), M\right)\right\}<\epsilon, \forall k \geq \tau
$$

for every stochastic motion $\left\{\phi\left(k, \omega, y_{0}, \mu_{0}^{N}\right), k \geq 0\right\} \in \mathfrak{s}$ where $d\left(y_{0}, M\right)<\delta$. 
The following lemma shows that the stability of a particular set $\mathfrak{M}$, such that $\mathfrak{M}$ is s-invariant, leads to conditions on only the equilibrium point $x_{e}=0$ of the embedded jump linear closed-loop system.

Lemma III.2.1 Consider the stochastic dynamical system $\left\{\mathbb{Z}^{+}, Y, d, \mathfrak{s}\right\}$ defined for a HJLS. The set $\mathfrak{M} \triangleq\left\{\left[x_{e}^{\mathrm{T}} z^{\mathrm{T}}\right]^{\mathrm{T}} \in Y \mid x_{e}=0 \in \mathbb{R}^{n}, z \in \Sigma_{S}\right\}$, when $k_{0}=0$, is

- stable in the second mean if for any $\epsilon>0$ there exists $\delta=\delta(\epsilon)>0$ such that

$$
\mathbf{E}\left\{\|x(k)\|^{2}\right\}<\epsilon, \forall k \geq 0
$$

for any stochastic motion $\left\{\phi\left(k, \omega, y_{0}, \mu_{0}^{N}\right), k \geq 0\right\} \in \mathfrak{s}$ where $\left\|x_{0}\right\|<\delta$.

- asymptotically stable in the second mean if it is stable in the second mean and if for any $\epsilon>0$ there exists $\delta=\delta(\epsilon)>0$ and $\tau=\tau(\epsilon)$ such that

$$
\mathbf{E}\left\{\|x(k)\|^{2}\right\}<\epsilon, \forall k \geq \tau
$$

for any stochastic motion $\left\{\phi\left(k, \omega, y_{0}, \mu_{0}^{N}\right), k \geq 0\right\} \in \mathfrak{s}$ where $\left\|x_{0}\right\|<\delta$.

Proof: Both results follow immediately by noting that $\mathbf{E}\left\{d^{2}\left(\phi\left(k, \omega, y_{0}, \mu_{0}^{N}\right), \mathfrak{M}\right)\right\}=$ $\mathbf{E}\left\{\|x(k)\|^{2}\right\}$

Note that condition (19) is equivalent to requiring that $\lim _{k \rightarrow \infty} \mathbf{E}\left\{\|\boldsymbol{x}(k)\|^{2}\right\}=0$. This condition is called mean square stability (see Definition III.2.7). Lemma III.2.1 indicates that the asymptotic stability in the second mean of $\mathfrak{M}$, an invariant set of the HJLS, is equivalent to the mean square stability of $x_{e}=0$, an equilibrium point of the jump linear closed-loop system embedded in the HJLS. So, without loss of generality, consider the following stability definitions for $\mathfrak{M}$, written in terms of $x_{e}=0$ only. 
Definition III.2.7 (Second Moment Stability) Consider the stochastic dynamical system $\left\{\mathbb{Z}^{+}, Y, d, \mathfrak{S}\right\}$ defined for a HJLS. The equilibrium $x_{e}=0$ of the jump linear closed-loop system, or simply the HJLS, is said to be

- stochastically stable if, for every stochastic motion in $\mathfrak{S}$,

$$
\sum_{k=0}^{\infty} \mathbf{E}\left\{\|\boldsymbol{x}(k)\|^{2}\right\}<\infty ;
$$

- mean square stable if, for every stochastic motion in $\mathfrak{S}$,

$$
\mathbf{E}\left\{\|\boldsymbol{x}(k)\|^{2}\right\} \rightarrow 0 \text { as } k \rightarrow \infty
$$

- exponentially mean square stable if there exist real numbers $\lambda \in(0,1)$ and $\mu>0$ such that for all $k \geq 0$ and every stochastic motion in $\mathfrak{S}$

$$
\mathbf{E}\left\{\|\boldsymbol{x}(k)\|^{2}\right\} \leq \mu \lambda^{k}\left\|x_{0}\right\|^{2}
$$

- second moment stable if the HJLS is simultaneously stochastically, exponentially mean square, and mean square stable; and

- second moment unstable if the HJLS is simultaneously stochastically, exponentially mean square, and mean square unstable.

Remark III.2.2 The relationship between these definitions is well-known. For example, it is easy to see that stochastic and exponential mean square stability each imply mean square stability. Hence, mean square instability implies second moment instability. Also, note that exponential stability implies stochastic stability since $\sum_{k=0}^{\infty} \mathbf{E}\left\{\|\boldsymbol{x}(k)\|^{2}\right\} \leq \sum_{k=0}^{\infty} \mu \lambda^{k}\left\|x_{0}\right\|^{2}<\infty$. Hence, exponential mean square stability implies second moment stability. Finally, note that when a HJLS represents a Markov jump linear system, it is stochastically, exponentially or mean square stable if and only if it is second moment stable (Ji, Chizeck, Feng \& Loparo 1991). 
Remark III.2.3 In Definition III.2.7, conditions (20), (21), and (22) must hold for every stochastic motion of $\mathfrak{S}$. That is, they must hold for any $x_{0} \in \mathbb{R}^{n}$ and any initial distribution $\mu_{0}^{N}$. This in turn implies that the second moment stability of a HJLS should be independent of its initial state and the initial distribution of the Markov chain $\{\boldsymbol{N}(k), k \geq 0\}$. This fact is usually emphasized by some authors by including the phrase “...for any $x_{0} \in \mathbb{R}^{n}$ and any initial distribution $\mu_{0}^{N} \ldots$ " in their definitions of second moment stability (cf. Costa et al. 2005, Fang, Loparo 8 Feng 1995).

The independence from the initial distribution is important because $\{N(k), k \geq 0\}$ may include more than one ergodic class (see Papoulis 1991). Thus, it is possible for the second moment, $\mathbf{E}\left\{\|\boldsymbol{x}(k)\|^{2}\right\}$, to converge to zero when $\boldsymbol{N}(0)$ belongs to one ergodic class and to diverge to infinity when $\boldsymbol{N}(0)$ belongs to another class. This is clearly illustrated in (Example 2.2, Fang et al. 1995).

Remark III.2.4 In the rest of this chapter, each lemma and theorem will be careful to specify the underlying stochastic dynamical system under consideration. It will be implicitly understood that the stability results introduced by these lemmas and theorems are derived for the invariant set $\mathfrak{M}$. However, any reference to this particular set will be dropped. Finally, unless otherwise stated, it will be understood that the HJLS under consideration has the general form of Definition II.2.3.

The stability analysis framework has been completely introduced. The next section will derive the first testable sufficient conditions for MSS by analyzing HJLS's as switched systems. 


\section{III.3 STABILITY ANALYSIS OF HJLS'S USING THE SWITCHED SYSTEMS APPROACH}

\section{III.3.1 Preliminaries}

Consider the following autonomous linear discrete-time switched system

$$
x_{\vartheta}(k+1)=A_{\vartheta(k)} x_{\vartheta}(k), x_{\vartheta}(0)=x_{0}
$$

where $\{\vartheta(k), k \geq 0\} \in \mathcal{I}_{\ell_{\vartheta}}^{\infty}$ represents a discrete-time switching signal. At every time $k \geq 0, \vartheta(k)$ is used to select $A_{\vartheta(k)} \in \mathfrak{A} \triangleq\left\{A_{i}: A_{i} \in \mathbb{R}^{n \times n}, i \in \mathcal{I}_{\ell_{\vartheta}}\right\}$. The effect of the switching signals $\{\vartheta(k), k \geq 0\} \in \mathcal{I}_{\mathcal{\ell}_{\vartheta}}^{\infty}$ can also be seen via the set of discrete linear inclusions (DLI) consisting of the set of all possible trajectories

$$
\mathrm{DLI}(\mathfrak{A}) \triangleq\left\{\left(x_{\vartheta}(0), \ldots, x_{\vartheta}(k), \ldots\right): x_{\vartheta}(k) \in \mathbb{R}^{n}, k \in \mathbb{Z}^{+}\right\}
$$

where $x_{\vartheta}(k+1)=A_{\vartheta(k)} x_{\vartheta}(k), A_{\vartheta(k)} \in \mathfrak{A}$, and $\{\vartheta(k), k \geq 0\} \in \mathcal{I}_{\ell_{\vartheta}}^{\infty}$.

The stability of (23) has been studied by many authors by paralleling the approaches taken for continuous-time switched systems (cf. Molchanov \& Pyatnitskiy 1989, Gurvits 1995, Liberzon, Hespanha \& Morse 1995, Daafouz, Riedinger \& Iung 2002). One of the main goals of these studies is the derivation of conditions under which the trajectories of (23) converge to zero under any switched signal $\{\vartheta(k), k \geq 0\}$. This stability notion, called absolute asymptotic stability, is defined next.

Definition III.3.1 (Gurvits 1995) The equilibrium point $x_{\vartheta}=0$ of the discretetime switched system (23) is Absolutely Asymptotically Stable (AAS) if every trajectory in $D L I(\mathfrak{A})$ satisfies $\lim _{k \rightarrow \infty} x_{\vartheta}(k)=0$. 
Note that AAS can be studied by analyzing the limit behavior of infinite products of matrices from $\mathfrak{A}$. This is justified by the fact that (23) is AAS if and only if

$$
\lim _{k \rightarrow \infty} A_{\vartheta(k)} \cdots A_{\vartheta(0)}=0, \text { for all }\{\vartheta(k), k \geq 0\} \in \mathcal{I}_{\ell_{\vartheta}}^{\infty}
$$

In order to relate switched systems to HJLS's, consider the set of switching signals that is formed by the sample paths of a stochastic processes, $\{\tilde{\boldsymbol{\theta}}(k), k \geq 0\}$, with state space $\mathcal{I}_{\ell_{\vartheta}}$ and initial distribution $\mu_{0}^{\bar{\theta}}$. Also consider the system

$$
\tilde{\boldsymbol{x}}(k+1)=A_{\tilde{\boldsymbol{\theta}}(k)} \tilde{\boldsymbol{x}}(k), \quad \tilde{\boldsymbol{x}}(0)=\tilde{\boldsymbol{x}}_{0},
$$

where $A_{\tilde{\boldsymbol{\theta}}(k)} \in \mathfrak{A}$, and extend the mean square stability definition in Definition III.2.7 as follows.

Definition III.3.2 The system (24) is mean square stable if for every $x_{0}$ and $\mu_{0}^{\bar{\theta}}$ it follows that $\mathbf{E}\left\{\|\tilde{\boldsymbol{x}}(k)\|^{2}\right\} \rightarrow 0$ as $k \rightarrow \infty$.

Clearly, if (23) is absolutely asymptotically stable then (24) is mean square stable since for every sample path generated by $\tilde{\boldsymbol{\theta}}(k)$ it follows that $\lim _{k \rightarrow \infty} A_{\vartheta(k)} \cdots A_{\vartheta(0)}=$ 0 . This can be formalized as follows.

Lemma III.3.1 If (23) is absolutely asymptotically stable then (24) is mean square stable provided that $\boldsymbol{x}_{0}$ is a second order random variable.

Proof: Observe that $\{\tilde{\boldsymbol{\theta}}(k), k \geq 0\}$ can be viewed as a random element from $\Omega$ into $\mathcal{I}_{\ell_{\vartheta}}^{\infty}$. Thus, the AAS of (23) implies that for any $\epsilon>0$ and any $\omega \in \Omega,\|x(k, \omega)\|^{2} \leq \epsilon$ provided that $k$ is large enough. This in turn implies that ${ }^{1}$

$$
\left|\mathbf{E}\left\{\|\tilde{\boldsymbol{x}}(k)\|^{2}\right\}\right|=\mathbf{E}\left\{\|\tilde{\boldsymbol{x}}(k)\|^{2}\right\}=\int_{\Omega}\|x(k, \omega)\|^{2} \mathrm{dPr} \leq \int_{\Omega} \epsilon \mathrm{dPr}=\epsilon .
$$

\footnotetext{
${ }^{1}$ In the sequel, $\int$ represents Lebesgue integration. See also Appendix A.
} 
That is, $\left|\mathbf{E}\left\{\|\tilde{\boldsymbol{x}}(k)\|^{2}\right\}\right|<\epsilon$ for any arbitrary $\epsilon>0$ provided that $k$ is large enough. This last statement implies that $\lim _{k \rightarrow \infty} \mathbf{E}\left\{\|\tilde{\boldsymbol{x}}(k)\|^{2}\right\}=0$. Note that this conclusion is true independent of $x_{0}$ and $\mu_{0}^{\bar{\theta}}$. Hence (24) is MSS.

The stochastic process $\{\tilde{\boldsymbol{\theta}}(k), k \geq 0\}$ in (24) can be generated, for example, by a stochastically driven high level supervisor as in (4). In this light it is easy to see that the stability of hybrid jump linear systems can be studied with the tools available for discrete-time linear switched systems. The next subsection presents a new testable sufficient condition for the AAS of (23), Theorem III.3.3. By extension, this test applies also to hybrid jump linear systems.

\section{III.3.2 Stability Analysis}

A recent result in (Daafouz et al. 2002) provides a sufficient condition for the absolutely asymptotic stability of (23). This result, however, requires the solution of $\left(\ell_{\vartheta}\right)^{2}$ linear matrix inequalities (LMI's) in $\ell_{\vartheta}$ unknowns. The following theorem reduces the number of inequalities to only $\ell_{\vartheta}$. In the sequel, $P>0$ will denote a real, symmetric, and positive definite matrix in $\mathbb{R}^{n \times n}$.

Theorem III.3.1 If for a given set of matrices $\left\{W_{i}>0: i \in \mathcal{I}_{\ell_{\vartheta}}\right\}$, there exists a set of matrices $\left\{P_{i}>0: i \in \mathcal{I}_{\ell_{\vartheta}}\right\}$ satisfying the condition

$$
P_{i}=W_{i}+\sum_{j=0}^{\ell_{\vartheta}-1} A_{i}^{\mathrm{T}} P_{j} A_{i}, \forall i \in \mathcal{I}_{\ell_{\vartheta}}
$$

then (23) is absolutely asymptotically stable. 
Proof: Suppose (25) holds and observe for any $x \in \mathbb{R}^{n}$ that $x^{\mathrm{T}} A_{i}^{\mathrm{T}} P_{i} A_{i} x \geq 0$. Then

$$
\begin{aligned}
x^{\mathrm{T}} A_{i}^{\mathrm{T}} P_{j} A_{i} x-x^{\mathrm{T}} P_{i} x & \leq \sum_{j=0}^{\ell_{\vartheta}-1} x^{\mathrm{T}} A_{i}^{\mathrm{T}} P_{j} A_{i} x-x^{\mathrm{T}} P_{i} x \\
& \leq-x^{\mathrm{T}} W_{i} x,
\end{aligned}
$$

and therefore $P_{i}-A_{i}^{\mathrm{T}} P_{j} A_{i}>0$ for every $i, j \in \mathcal{I}_{\ell_{\vartheta}}$. Since the matrices $P_{i}$ satisfy the hypothesis in (Theorem 2, Daafouz et al. 2002), then system (23) is absolutely asymptotically stable.

The Ricatti equation (25) has been used to determine the MS stability of MJLS (Ji \& Chizeck 1990a). But to the best of our knowledge, this is the first time they have been used to study general switched systems. Moreover, there exists a simple test to determine the existence of a solution to (25). The following test has been adapted from the more general results in (Proposition 6, Costa \& Fragoso 1993) and (Lemma 1, Kubrusly \& Costa 1985).

Theorem III.3.2 Let $\left\{A_{i} \in \mathbb{R}^{n \times n}: i \in \mathcal{I}_{\ell_{\vartheta}}\right\}$ be given and define $\overline{\mathcal{A}}$ as

$$
\overline{\mathcal{A}}=\left(E_{\ell_{\vartheta}} \otimes I_{n^{2}}\right) \operatorname{diag}\left(A_{0} \otimes A_{0}, \ldots, A_{\ell_{\vartheta}-1} \otimes A_{\ell_{\vartheta}-1}\right),
$$

where $E_{\ell_{\vartheta}}$ is an $\ell_{\vartheta} \times \ell_{\vartheta}$ matrix of ones, and $I_{n^{2}}$ is an $n^{2} \times n^{2}$ identity matrix. Then, for every set of matrices $\left\{W_{i}>0: i \in \mathcal{I}_{\ell_{\vartheta}}\right\}$ there exists a unique set of matrices $\left\{P_{i}>0: i \in \mathcal{I}_{\ell_{\vartheta}}\right\}$ that satisfy (25) if and only if the spectral radius of $\overline{\mathcal{A}}$ is less than $1(\rho(\overline{\mathcal{A}})<1)$.

A consequence of this theorem is a spectral radius test to determine the absolute asymptotic stability of a switched system. 
Theorem III.3.3 Consider the system (23), and let $\overline{\mathcal{A}}$ be defined as in (26). If $\rho(\overline{\mathcal{A}})<1$ then the system $(23)$ is absolutely asymptotically stable.

Proof: The result follows directly from Theorems III.3.1 and III.3.2.

Observe that the AAS condition $\rho(\overrightarrow{\mathcal{A}})<1$ in Theorem III.3.3 is similar to the sufficient condition for the mean square stability of the system

$$
\tilde{\boldsymbol{x}}(k+1)=\sqrt{\ell_{\vartheta}} A_{\tilde{\boldsymbol{\theta}}(k)} \tilde{\boldsymbol{x}}(k),
$$

where $\{\tilde{\boldsymbol{\theta}}(k), k \geq 0\}, \tilde{\boldsymbol{\theta}}(k) \in \mathcal{I}_{\ell_{\vartheta}}$, is a Markov chain with transition probabilities $p_{i, j}^{\bar{\theta}}=$ $1 / \ell_{\vartheta}$ (Costa \& Fragoso 1993). Thus, Theorem III.3.3 has the following interpretation: if the mean of the sample paths of (27) goes asymptotically to zero then so does every trajectory of (23). The converse claim, however, is not always true. It can be seen that if (23) is absolutely asymptotically stable then only the mean value of $\|x(k)\|^{2}$ over all possible trajectories must approach zero asymptotically. This motivates our next result.

Theorem III.3.4 Let $\overline{\mathcal{A}}$ be defined as in (26). A necessary condition for (23) to be absolutely asymptotically stable is that $\rho(\overline{\mathcal{A}})<\ell_{\vartheta}$.

Note that a given switched system which fails the test in Theorem III.3.3 can still be AAS. Although more sophisticated methods are available, this criterion provides a simple first check.

The proof of Theorem III.3.4 parallels the necessity proof of (Theorem 2.1, Ji \& Chizeck 1990b). The asymptotic stability of (23) is used to show that for any given set of matrices $\left\{W_{i}>0: i \in \mathcal{I}_{\ell_{\vartheta}}\right\}$, there exists a unique set of matrices $\left\{P_{i}>0: i \in \mathcal{I}_{\ell_{\vartheta}}\right\}$ 
satisfying (25). That is,

$$
P_{i}=W_{i}+\left(1 / \ell_{\vartheta}\right) \sum_{j=0}^{\ell_{\theta}-1} A_{i}^{\mathrm{T}} P_{j} A_{i}
$$

This fact and Theorem III.3.2 give the desired result.

Proof of Theorem III.3.4: It is assumed that (23) is AAS. Let the initial condition $x_{0}$ be fixed and let the transition matrix $\Phi(k+n, k)$ be given by

$$
\Phi(k+l, k) \triangleq \begin{cases}I_{l}, & l=0 \\ A_{\vartheta(k+l-1)} \cdots A_{\vartheta(k)}, & l \geq 1\end{cases}
$$

Let $\left\{W_{i}>0 ; i \in \mathcal{I}_{\ell_{\vartheta}}\right\}$ be given and define $g(\Phi(k+n, k)) \triangleq \Phi^{\mathrm{T}}(k+l, k) W_{\vartheta(k+l)} \Phi(k+$ $l, k)$. Note that for every fixed value of $\vartheta(k), g(\Phi(k+l, k))$ can take on $\left(\ell_{\vartheta}\right)^{n}$ different values, one for each possible permutation of $\{\vartheta(k+1), \ldots, \vartheta(k+l)\}$. That is, if $\vartheta(k)=i$ and $l \geq 1$, then $g(\Phi(k+l, k))$ can take any of $\left(\ell_{\vartheta}\right)^{l}$ different values which are obtained by evaluating

$$
g(\Phi(k+l, k))=A_{i}^{\mathrm{T}} A_{\vartheta(k+1)}^{\mathrm{T}} \cdots A_{\vartheta(k+l-1)}^{\mathrm{T}} W_{\vartheta(k+l)} A_{\vartheta(k+l-1)} \cdots A_{\vartheta(k+1)} A_{i}
$$

for all the different values of $\{\vartheta(k+1), \ldots, \vartheta(k+l)\}$ (that is, $(0,0, \ldots, 0,0)$, $\left.(1,0, \ldots, 0,0), \ldots,\left(\ell_{\vartheta}-1, \ell_{\vartheta}-1, \ldots, \ell_{\vartheta}-1, \ell_{\vartheta}-1\right)\right)$. Thus, when $\vartheta(k)=i$, the average value of $g(\Phi(k+l, k))$ is defined as

$$
\hat{\mathrm{E}}\{g(\Phi(k+l, k)) \mid \vartheta(k)=i\} \triangleq \begin{cases}W_{i}, & l=0 \\ \sum_{\vartheta(k+1)=0}^{\ell_{\vartheta}-1} \ldots \sum_{\vartheta(k+l)=0}^{\ell_{\vartheta}-1} \frac{g\left(\Phi(k+n, k+1) A_{i}\right)}{\left(\ell_{\vartheta}\right)^{2}}, & l \geq 1 .\end{cases}
$$

Now, $W_{\vartheta(k+l)}$ is positive definite, so Rayleigh's inequality yields $\|g(\Phi(k+l, k))\| \leq$ $\bar{\lambda}\|\Phi(k+l, k)\|^{2}$, where $\bar{\lambda}=\max _{i}\left\{\lambda_{\max }\left(W_{i}\right)\right\}$, and $\lambda_{\max }\left(W_{i}\right)$ denotes the maximum eigenvalue of $W_{i}$. Furthermore, since (23) is ASS, it follows from (Theorem 22.7, 
Rugh 1996) that there exist scalars $\beta>1$ and $0<\alpha<1$ such that $\|\Phi(k+l, k)\|^{2} \leq$ $\beta \alpha^{l}$. Consequently,

$$
\|\hat{\mathrm{E}}\{g(\Phi(k+l, k)) \mid \vartheta(k)\}\| \leq \bar{\lambda} \beta \alpha^{l}
$$

Next, define the matrices $P(l, \vartheta(k)=i), l \geq 0$, as follows

$$
P(l, \vartheta(k)=i) \triangleq \sum_{i=k}^{k+l} \hat{\mathrm{E}}\{g(\Phi(i, k)) \mid \vartheta(k)=i\}
$$

and observe that they are symmetric and positive definite by definition. Furthermore, for any $m>l \geq 0$

$$
\begin{aligned}
\|P(m, \vartheta(k)=i)-P(l, \vartheta(k)=i)\| & \leq \sum_{i=k+l+1}^{k+m}\|\hat{\mathrm{E}}\{g(\Phi(i, k)) \mid \vartheta(k)\}\| \\
& \leq \sum_{i=l+1}^{m} \bar{\lambda} \beta \alpha^{i} \\
& \leq \bar{\lambda} \beta \alpha^{l+1} /(1-\alpha) .
\end{aligned}
$$

Since for any $\epsilon>0$ there exists a sufficiently large $l$ such that $\bar{\lambda} \beta \alpha^{l+1} /(1-\alpha)<$ $\epsilon$, then $P(l, \vartheta(k)=i)$ has a unique limit with respect to $l$. Hence, define $P_{i} \triangleq$ $\lim _{l \rightarrow \infty} P(l, \vartheta(k)=i)$, and observe that

$$
P(l, \vartheta(0)=i)=\sum_{\iota=0}^{l} \hat{\mathrm{E}}\{g(\Phi(\iota, 0)) \mid \vartheta(0)=i\}=W_{i}+\sum_{\iota=1}^{l} \hat{\mathrm{E}}\{g(\Phi(\iota, 0)) \mid \vartheta(0)=i\}
$$

In addition, since $g\left(\Phi(k+l, k+1) A_{\vartheta(k)}\right)=A_{\vartheta(k)}^{\mathrm{T}} g(\Phi(k+l, k+1)) A_{\vartheta(k)}$, then

$$
\hat{\mathrm{E}}\{g(\Phi(\iota, 0)) \mid \vartheta(0)=i\}=\left(1 / \ell_{\vartheta}\right) A_{i}^{\mathrm{T}} \sum_{j=0}^{\ell_{\vartheta}-1} \hat{\mathrm{E}}\{g(\Phi(\iota, 1)) \mid \vartheta(1)=j\} A_{i} .
$$

Substituting (30) in (29) gives

$$
P(l, \vartheta(0)=i)=W_{i}+\left(1 / \ell_{\vartheta}\right) \sum_{j=0}^{\ell_{\vartheta}-1} A_{i}^{\mathrm{T}} P(l-1, \vartheta(1)=j) A_{i} .
$$


Letting $l \rightarrow \infty$ in the expression above gives

$$
P_{i}=W_{i}+\left(1 / \ell_{\vartheta}\right) \sum_{j=0}^{\ell_{\vartheta}-1} A_{i}^{\mathrm{T}} P_{j} A_{i} .
$$

This implies that the matrices $P_{i}$ uniquely satisfy (28). Consequently, Theorem III.3.2 implies that $\rho\left(\overline{\mathcal{A}} / \ell_{\vartheta}\right)<1$ or $\rho(\overline{\mathcal{A}})<\ell_{\vartheta}$, completing the proof.

A HJLS whose associated jump linear system satisfies the conditions of Theorem III.3.3 is both absolutely asymptotically stable and mean square stable. This is formalized next.

Theorem III.3.5 Consider the stochastic dynamical system $\left\{\mathbb{Z}^{+}, Y, d, \mathfrak{S}\right\}$ defined for a HJLS. Let $\mathcal{A}$ be given by

$$
\mathcal{A} \triangleq\left(E_{\ell_{O}} \otimes I_{n^{2}}\right) \operatorname{diag}\left(A_{0} \otimes A_{0}, \ldots, A_{\ell_{O}-1} \otimes A_{\ell_{O}-1}\right)
$$

where $A_{i}, i \in \mathcal{I}_{\ell_{O}}$, are the state transition matrices of the embedded jump linear closed-loop system. If $\rho(\mathcal{A})<1$, the HJLS is $A A S$ and mean square stable.

Proof: This is a direct consequence of Theorem III.3.3 and Lemma III.3.1.

Observe that the conditions in Theorem III.3.5 imply that $\|x(k, \omega)\| \rightarrow 0$ as $k \rightarrow \infty$ for every $\omega \in \Omega$. Note, however, that if $\|x(k, \omega)\| \rightarrow \infty$ for some subset $\varsigma \subset \Omega, \mathbf{E}\left\{\|\boldsymbol{x}(k)\|^{2}\right\}$ can still converge to zero provided that, for instance, $\operatorname{Pr}\{\varsigma\}=0$. Moreover, depending on the structure of the supervisor, the sample paths of the output process $\{\boldsymbol{\theta}(k), k \geq 0\}$ may reside in a proper subset of $\mathcal{I}_{\ell_{0}}^{\infty}$, that is, only some (not all) the switching signals may drive the jump linear system embedded in the HJLS. Both situations show that the conditions in Theorem III.3.5 are conservative. The next section will derive less conservative conditions using singular value techniques. 


\section{III.4 STABILITY ANALYSIS OF HJLS'S USING SINGULAR VALUE TECHNIQUES}

Consider again a hybrid jump linear system as described in Definition II.2.3. That is, a HJLS with dynamics given by

$$
\begin{aligned}
\boldsymbol{x}(k+1) & =A_{\boldsymbol{\theta}(k)} \boldsymbol{x}(k), \quad \boldsymbol{x}(0)=\boldsymbol{x}_{0} \\
\boldsymbol{z}(k+1) & =S_{\boldsymbol{N}(k), \psi(\boldsymbol{x}(k))} \boldsymbol{z}(k) \\
\psi(x) & =\sum_{i=0}^{\ell_{\nu}-1} i \mathbf{1}_{\left\{x \in R_{i}\right\}}, \quad \bigcup_{i \in \mathcal{I}_{\ell_{\nu}}} R_{i}=\mathbb{R}^{n} .
\end{aligned}
$$

This section will provide a MSS condition for such systems based on the maximum and minimum singular values of the state transition matrices $A_{i}, i \in \mathcal{I}_{\ell_{0}}$. This section also includes a detailed analysis of the stability of a HJLS with performance supervision. In the sequel, $\bar{\sigma}(A)$ and $\underline{\sigma}(A)$ will denote the maximum and minimum singular values of the matrix $A$.

Theorem III.4.1 Consider the stochastic dynamical system $\left\{\mathbb{Z}^{+}, Y, d, \mathfrak{S}\right\}$ defined for a HJLS. If $\bar{\sigma}\left(A_{i}\right)<1, \forall i \in \mathcal{I}_{\ell_{O}}$, then the HJLS is second moment stable. If $\underline{\sigma}\left(A_{i}\right)>1$, $\forall i \in \mathcal{I}_{\ell_{O}}$, then the HJLS is second moment unstable.

Proof: Consider the sample paths of the jump linear closed-loop system. Note that the state of the jump linear system, $\boldsymbol{x}(k)$, has the form $\boldsymbol{x}(k)=A_{\boldsymbol{\theta}(k-1)} A_{\boldsymbol{\theta}(k-2)} \ldots A_{\boldsymbol{\theta}(0)} x_{0}$ for any stochastic motion in $\mathfrak{S}$ with fixed initial state $y_{0}=\left[\begin{array}{ll}x_{0}^{\mathrm{T}} & z_{0}^{\mathrm{T}}\end{array}\right]^{\mathrm{T}}$. Hence, $\|\boldsymbol{x}(k)\|=$ $\left\|A_{\boldsymbol{\theta}(k-1)} A_{\boldsymbol{\theta}(k-2)} \ldots A_{\boldsymbol{\theta}(0)} x_{0}\right\|$. This expression can be bounded using singular values as 
follows:

$$
\begin{aligned}
\underline{\sigma}\left(A_{\boldsymbol{\theta}(k-1)} A_{\boldsymbol{\theta}(k-2)} \ldots A_{\theta(0)}\right)\left\|x_{0}\right\| & \leq\|\boldsymbol{x}(k)\| \leq \bar{\sigma}\left(A_{\boldsymbol{\theta}(k-1)} A_{\boldsymbol{\theta}(k-2)} \ldots A_{\boldsymbol{\theta}(0)}\right)\left\|x_{0}\right\| \\
\underline{\sigma}\left(A_{\boldsymbol{\theta}(k-1)}\right) \cdots \underline{\sigma}\left(A_{\boldsymbol{\theta}(1)}\right) \underline{\sigma}\left(A_{\theta(0)}\right)\left\|x_{0}\right\| & \leq\|\boldsymbol{x}(k)\| \leq \bar{\sigma}\left(A_{\boldsymbol{\theta}(k-1)}\right) \cdots \bar{\sigma}\left(A_{\boldsymbol{\theta}(1)}\right) \bar{\sigma}\left(A_{\theta(0)}\right)\left\|x_{0}\right\| \\
\prod_{i=0}^{k-1} \underline{\sigma}\left(A_{\boldsymbol{\theta}(i)}\right)\left\|x_{0}\right\| & \leq\|\boldsymbol{x}(k)\| \leq \prod_{i=0}^{k-1} \bar{\sigma}\left(A_{\boldsymbol{\theta}(i)}\right)\left\|x_{0}\right\| \\
\min _{i \in \mathcal{I}_{\ell_{O}}}\left\{\underline{\sigma}\left(A_{i}\right)\right\}^{k}\left\|x_{0}\right\| & \leq\|\boldsymbol{x}(k)\| \leq \max _{i \in \mathcal{I}_{\ell_{O}}}\left\{\bar{\sigma}\left(A_{i}\right)\right\}^{k}\left\|x_{0}\right\| .
\end{aligned}
$$

Suppose that $\bar{\sigma}\left(A_{i}\right)<1$ for all $i \in \mathcal{I}_{\ell_{O}}$. Let $\mu=1$ and $\lambda=\max _{i \in \mathcal{I}_{\ell_{O}}}\left\{\bar{\sigma}\left(A_{i}\right)\right\}^{2}<1$, and observe that $\|\boldsymbol{x}(k)\|^{2} \leq \mu \lambda^{k}\left\|x_{0}\right\|^{2}$ and $\mathbf{E}\left\{\|\boldsymbol{x}(k)\|^{2}\right\} \leq \mu \lambda^{k}\left\|x_{0}\right\|^{2}$. Clearly, the HJLS is exponentially mean square stable and, therefore, second moment stable.

Now suppose that $g\left(A_{i}\right)>1$ for all $i \in \mathcal{I}_{\ell_{O}}$. Using the same reasoning as above, it follows that $\min _{i \in \mathcal{I}_{\ell_{O}}}\left\{\underline{\sigma}\left(A_{i}\right)\right\}^{2 k}\left\|x_{0}\right\|^{2} \leq \mathbf{E}\left\{\|\boldsymbol{x}(k)\|^{2}\right\}$. Since $\min _{i \in \mathcal{I}_{\ell_{O}}}\left\{\underline{\sigma}\left(A_{i}\right)\right\}^{2 k}\left\|x_{0}\right\|^{2}$ is a divergent sequence, $\mathbf{E}\left\{\|\boldsymbol{x}(k)\|^{2}\right\} \rightarrow \infty$ as $k \rightarrow \infty$ and the result follows.

This theorem is a discrete-time generalization of Theorem 1 in (Mitra, Tarn \& Dai 2001) for switched systems. Less conservative tests can be derived for specific HJLS's by taking their particular structure into account. This is demonstrated in the next subsection, where a simple HJLS with performance supervision is analyzed. The material also motivates the use of finite-time stability concepts.

\section{III.4.1 Stability Analysis of a Simple HJLS with Performance Supervi- sion}

In safety critical systems, the saturation of certain signals or a critical event is often detected by a threshold test on the state's norm. If such events causes the jump linear closed-loop system to become unstable, the role of the supervisor would be to 
command a switch to a fail-safe operating mode. That is, the supervisor reacts to the lack of stability in the closed-loop system (reflected by a big increase in the state's norm) by changing its operating mode. In addition, the supervisor may also activate a fail-safe operating mode in response to an external fault-detection signal.

The focus here, however, is on the case where the supervisor does not command such a fail-safe operation. The effect of the fault-detection signal or the statedependent input when the performance boundary is reached is simply to reflect the change of models for the jump linear closed-loop system. Under these conditions, it is not expected that the HJLS will perform satisfactorily once the performance boundary is reached. This is formally shown in Theorem III.4.3, where this specific HJLS is shown to be second moment unstable. Despite the limited application of this HJLS, this is a useful problem to analyze because it shows how the structure of the HJLS can be exploited to derive stability results. It also shows how complex system behavior can be represented by a HJLS with performance supervision. Finally, since stability over an infinite-time horizon is not expected, it is useful to consider an alternative notion of stability, finite-time stability, as first proposed in (LaSalle \& Lefschetz 1961) and used, for example, in (Michel \& Porter 1972, Ye \& Michel 1998, Zhai \& Michel 2002, Xu \& Antsaklis 2003). Two such definitions and a corresponding sufficient stability condition are given at the end of this subsection. The precise structure of the system of interest is given first.

Assume that the fault-detection signal is modeled by a two-state, homogeneous Markov chain $\{N(k), k \geq 0\}$ with initial distribution $\mu_{0}^{N}=\left[p_{0}^{N} p_{1}^{N}\right]=[\operatorname{Pr}\{N(0)=$ $0\} \operatorname{Pr}\{N(0)=1\}]$, and transition probability matrix $\Pi_{N}=\left[p_{i, j}^{N}\right], i, j \in\{0,1\}$. For 
simplicity, let $0<p_{0}^{N}, p_{1}^{N}<1$ and $\{N(k), k \geq 0\}$ be aperiodic with no absorbing states, that is, let $p_{i, i}^{N} \in(0,1), i \in\{0,1\}$. In this context, $N(k)=0(N(k)=1)$ signifies the absence (presence) of detected faults in the system at time $k$.

The analog to symbol map is used to detect whether the norm of the closed-loop system's state vector has reached a performance boundary given by $\alpha \in \mathbb{R}^{+}$, that is,

$$
\boldsymbol{\nu}(k)=\psi(\boldsymbol{x}(k))= \begin{cases}1, & \|x(k)\| \geq \alpha>0 \\ 0, & \|x(k)\|<\alpha\end{cases}
$$

Clearly, the role of the detector is to output a 1 when the performance boundary is exceeded. As described above, whenever $N(k)=1$ or $\nu(k)=1$ the supervisor always selects the same failure mode of operation. This behavior can be modeled with a HJLS as follows.

Consider system (32) and note that $\mathcal{I}_{\ell_{N}}=\mathcal{I}_{\ell_{O}}=\{0,1\}$. For simplicity, assume that $\boldsymbol{z}(k)$ and $\boldsymbol{\theta}(k)$ are isomorphic, that is, $\ell_{S}=2$, with $\boldsymbol{z}(k) \in\left\{e_{1}, e_{2}\right\}$. The output map, $\varpi$, has the form

$$
\begin{aligned}
\varpi: \Sigma_{S} & \rightarrow \mathcal{I}_{\ell_{O}} \\
\boldsymbol{z}(k) & \mapsto \boldsymbol{\theta}(k)=\varpi(\boldsymbol{z}(k))=\left[\begin{array}{ll}
0 & 1
\end{array}\right] \boldsymbol{z}(k) .
\end{aligned}
$$

To complete the model, observe that $\mathcal{I}_{\ell_{\nu}}=\{0,1\}$. Thus, the FSM's state transition matrices are given by $S_{0,0}=\left[\begin{array}{ll}1 & 1 \\ 0 & 0\end{array}\right]$ and $S_{0,1}=S_{1,0}=S_{1,1}=\left[\begin{array}{ll}0 & 0 \\ 1 & 1\end{array}\right]$.

Alternatively, the supervisor's actions can be represented by the state diagram in Figure 9. In this figure, the arcs in the transition diagram are labeled with the values of the input processes $\{(\boldsymbol{\nu}(k), \boldsymbol{N}(k)), k \geq 0\}$. (An $\mathrm{x}$ in the transition diagram represents a don't care condition). The nodes in Figure 9 correspond to the values of 


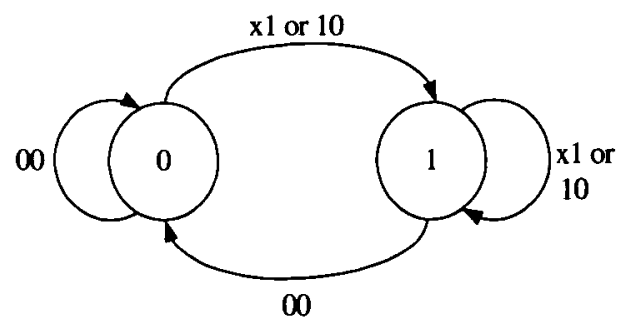

FIG. 9: Transition diagram for a finite state machine representation of a logical OR operation.

$\boldsymbol{\theta}(k) \in \mathcal{I}_{\ell_{O}}$. Based on this diagram, the evolution of $\boldsymbol{\theta}(k)$ can be expressed as follows:

$$
\begin{aligned}
\boldsymbol{\theta}(k+1) & =\left\{\begin{array}{cc}
1 & : \boldsymbol{\nu}(k)=1 \\
\boldsymbol{N}(k) & : \boldsymbol{\nu}(k)=0
\end{array}\right. \\
& =\boldsymbol{N}(k)+\boldsymbol{\nu}(k)(1-\boldsymbol{N}(k)) \\
& =\operatorname{OR}(\boldsymbol{N}(k), \boldsymbol{\nu}(k))
\end{aligned}
$$

The stability analysis of such a system can be done using its associated auxiliary scalar Markov jump linear system as defined below.

Definition III.4.1 An Auxiliary Scalar Markov Jump Linear System (asMJLS) associated with a HJLS is a scalar Markov jump linear system driven by the same stochastic input process, $\{\boldsymbol{N}(k), k \geq 0\}$, and represented by

$$
\tilde{\boldsymbol{x}}(k+1)=\tilde{a}_{N(k)} \tilde{\boldsymbol{x}}(k)
$$

where $\tilde{x}(0)=\tilde{x}_{0}$ and $\tilde{a}_{i} \in \mathbb{R}$ for all $i \in \mathcal{I}_{\ell_{N}}$.

In particular, the asMJLS associated with the HJLS specified by (34)-(35) has two modes, $\tilde{a}_{0}$ and $\tilde{a}_{1}$. It is assumed that $\tilde{a}_{0} \neq 0$, so that its sample solutions are of the form

$$
\tilde{\boldsymbol{x}}(k)=\left(\tilde{a}_{1} / \tilde{a}_{0}\right)^{\boldsymbol{n}(k)}\left(\tilde{a}_{0}\right)^{k} \tilde{x}_{0}
$$


where $\{\boldsymbol{n}(k), k \geq 1\}$, and $\boldsymbol{n}(k) \triangleq \sum_{i=0}^{k-1} \boldsymbol{N}(i), k \geq 1$, is the stochastic process that counts the number of ones in the sequence $\{N(0), \ldots, N(k-1)\}$. This framework enables the following result.

Theorem III.4.2 Consider the stochastic dynamical system $\left\{\mathbb{Z}^{+}, Y, d, \mathfrak{s}\right\}$ associated with the HJLS specified by (34)-(35), where $\mathfrak{s} \subset \mathfrak{S}$ is the collection of stochastic motions with initial state $y_{0}=\left[x_{0}^{\mathrm{T}} e_{1}^{\mathrm{T}}\right]^{\mathrm{T}}$, and $x_{0} \in \mathbb{R}^{n}$ with $\left\|x_{0}\right\|<\alpha$.

(a) If $\underline{\sigma}\left(A_{1}\right) / \underline{\sigma}\left(A_{0}\right)>1$ then the HJLS is second moment unstable whenever its associated asMJLS in (36) with $\tilde{a}_{0}=\underline{\sigma}\left(A_{0}\right)$ and $\tilde{a}_{1}=\underline{\sigma}\left(A_{1}\right)$ is second moment unstable.

(b) If $\bar{\sigma}\left(A_{0}\right) / \bar{\sigma}\left(A_{1}\right)>1$ then the HJLS is second moment stable whenever its associated asMJLS in (36) with $\tilde{a}_{0}=\bar{\sigma}\left(A_{0}\right)$ and $\tilde{a}_{1}=\bar{\sigma}\left(A_{1}\right)$ is second moment stable.

Proof: Let $\boldsymbol{m}(k) \triangleq \sum_{i=1}^{k-1} \boldsymbol{\theta}(i)$. Note that $z_{0}=e_{1}$, so $\boldsymbol{\theta}(0)=0$. A reasoning similar to the one used to derive (33) yields

$$
\begin{aligned}
\|\boldsymbol{x}(k)\| & \geq\left(\underline{\sigma}\left(A_{1}\right) / \underline{\sigma}\left(A_{0}\right)\right)^{\boldsymbol{m}(k)} \underline{\sigma}\left(A_{0}\right)^{k}\left\|x_{0}\right\| \\
\|\boldsymbol{x}(k)\| & \leq\left(\bar{\sigma}\left(A_{1}\right) / \bar{\sigma}\left(A_{0}\right)\right)^{\boldsymbol{m}(k)} \bar{\sigma}\left(A_{0}\right)^{k}\left\|x_{0}\right\| .
\end{aligned}
$$

To prove (a), assume $\underline{\sigma}\left(A_{1}\right) / \underline{\sigma}\left(A_{0}\right)>1$, and let $\tilde{a}_{0}=\underline{\sigma}\left(A_{0}\right), \tilde{a}_{1}=\underline{\sigma}\left(A_{1}\right)$, and $\tilde{x}_{0}=\underline{\sigma}\left(A_{0}\right)\left\|x_{0}\right\|$ in $(37)$. Thus,

$$
\tilde{\boldsymbol{x}}(k-1)=\left(\underline{\sigma}\left(A_{1}\right) / \underline{\sigma}\left(A_{0}\right)\right)^{\boldsymbol{n}(k-1)} \underline{\sigma}\left(A_{0}\right)^{k}\left\|x_{0}\right\| .
$$

Note from (35) that $\boldsymbol{\theta}(k+1) \geq \boldsymbol{N}(k)$, which in turn implies that $\boldsymbol{m}(k) \geq \boldsymbol{n}(k-1)$. This fact and (38) yield

$$
\|\boldsymbol{x}(k)\| \geq \tilde{\boldsymbol{x}}(k-1),
$$


and, consequently, $\mathbf{E}\left\{\|\boldsymbol{x}(k)\|^{2}\right\} \geq \mathbf{E}\left\{\tilde{\boldsymbol{x}}^{2}(k-1)\right\}$. Now, if (36) is mean square unstable then $\mathbf{E}\left\{\tilde{\boldsymbol{x}}^{2}(k-1)\right\} \rightarrow \infty$ as $k \rightarrow \infty$, and thus, $\mathbf{E}\left\{\|\boldsymbol{x}(k)\|^{2}\right\} \rightarrow \infty$ as $k \rightarrow \infty$. Hence, the HJLS is mean square unstable, making it also second moment unstable.

A similar argument proves (b). Assume $\bar{\sigma}\left(A_{0}\right) / \bar{\sigma}\left(A_{1}\right)>1$, and let $\tilde{a}_{0}=\bar{\sigma}\left(A_{0}\right)$, $\tilde{a}_{1}=\bar{\sigma}\left(A_{1}\right)$ and $\tilde{x}_{0}=\bar{\sigma}\left(A_{0}\right)\left\|x_{0}\right\|$ in (37). Then (39) reduces to

$$
\|\boldsymbol{x}(k)\| \leq \tilde{\boldsymbol{x}}(k-1)
$$

which implies that $\mathbf{E}\left\{\|\boldsymbol{x}(k)\|^{2}\right\} \leq \mathbf{E}\left\{\tilde{\boldsymbol{x}}^{2}(k-1)\right\}$. Now observe that if the MJLS in (36) is second moment stable then $\mathbf{E}\left\{\tilde{\boldsymbol{x}}^{2}(k-1)\right\} \leq \mu \lambda^{k-1}\left\|x_{0}\right\|^{2}$ for some $\mu>0$ and $\lambda \in(0,1)$. So it follows that $\mathbf{E}\left\{\|\boldsymbol{x}(k)\|^{2}\right\} \leq(\mu / \lambda) \lambda^{k}\left\|x_{0}\right\|^{2}$. Thus, the HJLS is exponentially mean square stable and consequently it is also second moment stable.

Note that Theorem III.4.2 requires less restrictive conditions than Theorem III.4.1, since it only requires the ratio of the singular values of $A_{0}$ and $A_{1}$ to be bigger than 1 . Also note that to determine the mean square stability of (36) it is enough to compute the spectral radius of the matrix

$$
a=\left[\begin{array}{l}
p_{0,0}^{N}\left(\tilde{a}_{0}\right)^{2} p_{1,0}^{N}\left(\tilde{a}_{1)^{2}}\right. \\
p_{0,1}^{N}\left(\tilde{a}_{0}\right)^{2} p_{1,1}^{N}\left(\tilde{a}_{1}\right)^{2}
\end{array}\right]
$$

If $\rho(a)<1$ then $(36)$ is mean square stable; otherwise, it is unstable (see Costa \& Fragoso 1993).

The following two lemmas are used to prove Theorem III.4.3. The ceiling operator $\lceil c\rceil$ represents the smallest integer greater than or equal to $c \in \mathbb{R}$.

Lemma III.4.1 Consider the asMJLS associated with the HJLS specified by (34)(35). If $0<\tilde{x}_{0}<\alpha, \tilde{a}_{0}<1$, and $\tilde{a}_{1}>1$ then the first sample time, $k^{*}$, for which $\tilde{\boldsymbol{x}}(k)$ may reach or exceed $\alpha$ is given by 


$$
k^{*}=\left\lceil\frac{\log \left(\alpha / \tilde{x}_{0}\right)}{\log \left(\tilde{a}_{1}\right)}\right\rceil \geq 1
$$

The probability that the worst case sample path, i.e. the sample path for which $N(k)=$ 1 for all $k>0$, reaches $\alpha$ at time $k=k^{*}$ is $\operatorname{Pr}\left\{\left|\tilde{\boldsymbol{x}}\left(k^{*}\right)\right| \geq \alpha\right\}=p_{1}^{N}\left(p_{1,1}^{N}\right)^{k^{*}-1}>0$. Furthermore, for every $k<k^{*}, \operatorname{Pr}\{|\tilde{\boldsymbol{x}}(k)| \geq \alpha\}=0$.

Proof: First, assume without loss of generality that $\mathrm{p}_{1}^{N}>0$. If $p_{1}^{N}=0$ then analyze the system as if it were starting at $k=1$ with initial state $\tilde{a}_{0} x_{0}<\alpha$ and $p_{1}^{N}=p_{0,1}^{N}>0$, which is not zero by assumption. Observe that $\tilde{x}_{0}<\alpha$, so $\tilde{\boldsymbol{x}}(k)$ can reach $\alpha$ only from below. Also, note that the worst case sample path is

$$
\max _{\omega \in \Omega}\{\tilde{\boldsymbol{x}}(k, \omega)\}=\left(\tilde{a}_{1}\right)^{k} \tilde{x}_{0}
$$

By definition, the first time at which $\tilde{\boldsymbol{x}}(k)$ could reach or exceed $\alpha$ is $k^{*}$. Thus, setting (42) equal to $\alpha$ and solving for $k^{*}$ gives equation (41). Now, observe from (37) that $\left|\boldsymbol{x}\left(k^{*}\right)\right| \geq \alpha$ only if $\boldsymbol{n}\left(k^{*}\right)=k^{*}$. Then, it follows that $\operatorname{Pr}\left\{\left|\boldsymbol{x}\left(k^{*}\right)\right| \geq \alpha\right\}=$ $\operatorname{Pr}\left\{N(i)=1, \forall i=0,1, \ldots, k^{*}-1\right\}=p_{1}^{N}\left(p_{1,1}^{N}\right)^{k^{*}-1}>0\left(\right.$ note that $p_{1}^{N}>0$ and $p_{1,1}^{N}>0$ by definition). Clearly, for every $k<k^{*},|\tilde{\boldsymbol{x}}(k)|<\alpha$ and $\operatorname{Pr}\{|\tilde{\boldsymbol{x}}(k)| \geq \alpha\}=0$.

The long term behavior of the sample paths of the HJLS specified by (34)-(35) is examined next under the multidimensional generalization of the conditions of Lemma III.4.1.

Lemma III.4.2 Consider the stochastic dynamical system $\left\{\mathbb{Z}^{+}, Y, d, \mathfrak{S}\right\}$ associated with the HJLS specified by (34)-(35). Let $\bar{\sigma}\left(A_{0}\right)<1, \underline{\sigma}\left(A_{1}\right)>1$ and $\underline{\sigma}\left(A_{0}\right) \underline{\sigma}\left(A_{1}\right)>1$. If for a stochastic motion in $\mathfrak{S}$ there exists sample paths such that $\left\|\boldsymbol{x}\left(k^{\prime}\right)\right\| \geq \alpha$ for 
some $k^{\prime} \geq 0$, then there exists a finite $k^{\prime \prime} \geq k^{\prime}$ such that for those sample paths $\|\boldsymbol{x}(k+1)\|>\|\boldsymbol{x}(k)\|>\alpha$ for all $k \geq k^{\prime \prime}$.

Proof: Note that $\boldsymbol{\theta}\left(k^{\prime}+1\right)=1$, since by assumption $\left\|\boldsymbol{x}\left(k^{\prime}\right)\right\| \geq \alpha$. Thus, it follows that

$$
\left\|\boldsymbol{x}\left(k^{\prime}+2\right)\right\|=\left\|A_{1} A_{\boldsymbol{\theta}\left(k^{\prime}\right)} \boldsymbol{x}\left(k^{\prime}\right)\right\| \geq \sigma\left(A_{1} A_{\boldsymbol{\theta}\left(k^{\prime}\right)}\right)\left\|\boldsymbol{x}\left(k^{\prime}\right)\right\|
$$

But $\underline{\sigma}\left(A_{1} A_{\boldsymbol{\theta}\left(k^{\prime}\right)}\right) \geq \underline{\sigma}\left(A_{1}\right) \underline{\sigma}\left(A_{\boldsymbol{\theta}\left(k^{\prime}\right)}\right), \underline{\sigma}\left(A_{\boldsymbol{\theta}\left(k^{\prime}\right)}\right) \geq \underline{\sigma}\left(A_{0}\right)$, and $\underline{\sigma}\left(A_{0}\right) \underline{\sigma}\left(A_{1}\right)>1$ (by assumption), so

$$
\left\|\boldsymbol{x}\left(k^{\prime}+2\right)\right\| \geq \underline{\sigma}\left(A_{1}\right) \underline{\sigma}\left(A_{0}\right)\left\|\boldsymbol{x}\left(k^{\prime}\right)\right\|>\left\|\boldsymbol{x}\left(k^{\prime}\right)\right\|
$$

In the same way, it can be shown that $\left\|\boldsymbol{x}\left(k^{\prime}+2 m\right)\right\|>\left\|\boldsymbol{x}\left(k^{\prime}+2 m-2\right)\right\|$ for all $m \geq 1$. Even though initially nothing can be said about $\left\|\boldsymbol{x}\left(k^{\prime}+m\right)\right\|$ when $m$ is odd, notice that $\left\|\boldsymbol{x}\left(k^{\prime}+2 m\right)\right\|$ is a strictly increasing subsequence. Thus, there exists $m^{\prime} \geq 1$ such that $\left\|\boldsymbol{x}\left(k^{\prime}+2 m^{\prime}\right)\right\|>\alpha / \underline{\sigma}\left(A_{0}\right)>\alpha$ and $\boldsymbol{\theta}\left(k^{\prime}+2 m^{\prime}+1\right)=1$. This in turn implies that

$$
\begin{aligned}
\left\|\boldsymbol{x}\left(k^{\prime}+2 m^{\prime}+1\right)\right\| & =\left\|A_{\boldsymbol{\theta}\left(k^{\prime}+2 m^{\prime}\right)} \boldsymbol{x}\left(k^{\prime}+2 m^{\prime}\right)\right\| \\
& \geq \underline{\sigma}\left(A_{\boldsymbol{\theta}\left(k^{\prime}+2 m^{\prime}\right)}\right)\left\|\boldsymbol{x}\left(k^{\prime}+2 m^{\prime}\right)\right\| \\
& \geq \underline{\sigma}\left(A_{0}\right)\left\|\boldsymbol{x}\left(k^{\prime}+2 m^{\prime}\right)\right\|>\alpha .
\end{aligned}
$$

Hence, for $k \geq k^{\prime \prime}=k^{\prime}+2 m^{\prime}+1, \boldsymbol{\theta}(k)=1$ so that $\|\boldsymbol{x}(k+1)\|>\|\boldsymbol{x}(k)\|>\alpha$ and the result then follows.

Theorem III.4.3 Consider the stochastic dynamical system $\left\{\mathbb{Z}^{+}, Y, d, \mathfrak{s}\right\}$ associated with the HJLS specified by (34)-(35), where $\mathfrak{s} \subset \mathfrak{S}$ is the collection of stochastic motions with initial state $y_{0}=\left[\begin{array}{ll}x_{0}^{\mathrm{T}} & e_{1}^{\mathrm{T}}\end{array}\right]^{\mathrm{T}}$, and $x_{0} \in \mathbb{R}^{n}$ with $\left\|x_{0}\right\|<\alpha$. Let $\bar{\sigma}\left(A_{0}\right)<1$, $\underline{\sigma}\left(A_{1}\right)>1$ and $\underline{\sigma}\left(A_{0}\right) \underline{\sigma}\left(A_{1}\right)>1$. The HJLS is second moment unstable. 
Proof: Let $\tilde{a}_{0}=\underline{\sigma}\left(A_{0}\right), \tilde{a}_{1}=\sigma\left(A_{1}\right)$ and $\tilde{x}_{0}=\underline{\sigma}\left(A_{0}\right)\left\|x_{0}\right\|$ in (36) and observe that $\underline{\sigma}\left(A_{1}\right) / \underline{\sigma}\left(A_{0}\right)>1$. This implies that (40) holds (see Theorem III.4.2). This also implies that the conditions of Lemma III.4.1 are satisfied, so there exist sample solutions of (36) such that at time $k^{\prime}-1=k^{*},\left|\tilde{x}\left(k^{\prime}-1\right)\right| \geq \alpha$ with $k^{*}$ defined as in (41).

Now, (40) indicates that there exists at least one stochastic motion in $\mathfrak{s}$ with sample paths that make $\left\|x\left(k^{\prime}\right)\right\| \geq \alpha$. This in turn implies, by Lemma III.4.2, that these sample paths make $\|\boldsymbol{x}(k)\| \geq \alpha$ for every $k \geq k^{\prime \prime} \geq k^{\prime}$. Hence, the Markov inequality implies that for all $k \geq k^{\prime \prime}>k^{*}$,

$$
\mathbf{E}\left\{\|\boldsymbol{x}(k)\|^{2}\right\} \frac{1}{\alpha^{2}} \geq \operatorname{Pr}\{\|\boldsymbol{x}(k)\| \geq \alpha\} \geq \operatorname{Pr}\left\{\tilde{\boldsymbol{x}}\left(k^{*}\right) \geq \alpha\right\}>0
$$

which in turn implies that $\lim _{k \rightarrow \infty} \mathbf{E}\left\{\|\boldsymbol{x}(k)\|^{2}\right\}>0$ and $\sum_{k=0}^{\infty} \mathbf{E}\left\{\|\boldsymbol{x}(k)\|^{2}\right\}=\infty$ for at least the aforementioned stochastic motion in $\mathfrak{s}$. This indicates that $\mathbf{E}\left\{\|\boldsymbol{x}(k)\|^{2}\right\}$ does not converge to zero for all the stochastic motions in $\mathfrak{s} \subset \mathfrak{S}$, which in turn implies that the HJLS is mean square and second moment unstable.

Clearly, over an infinite time horizon a HJLS satisfying the conditions in Theorem III.4.3 is mean square unstable since there are sample solutions that are ultimately unbounded. But if the HJLS were to operate only over finite time horizons, its sample paths would be bounded remaining below the performance boundary. The following definition from (Michel \& Porter 1972) formalizes the concept of finite-time stability.

Definition III.4.2 Consider the stochastic dynamical system $\left\{\mathbb{Z}^{+}, Y, d, \mathfrak{S}\right\}$ defined for a HJLS. Let $T \in \mathbb{Z}^{+}$and $\alpha, \beta \in \mathbb{R}^{+}$be constants such that $T>0$ and $\beta \geq \alpha>0$. The HJLS is said to be

(a) finite-time stable with respect to $(\alpha, \boldsymbol{\beta}, \boldsymbol{T})$ if $\|x(0)\|<\alpha$ implies that 
$\|\boldsymbol{x}(k)\| \leq \beta$ for all $k \in \mathcal{I}_{T+1}$, and

(b) mean square finite-time stable with respect to $(\alpha, \beta, T)$ if $\|x(0)\|<\alpha$ implies that $\mathbf{E}\left\{\|\boldsymbol{x}(k)\|^{2}\right\} \leq \beta^{2}$ for all $k \in \mathcal{I}_{T+1}$.

In this definition $\beta$ represents the performance boundary for all the sample solutions of a HJLS. The next result follows directly from Lemma III.4.1.

Lemma III.4.3 Consider the stochastic dynamical system $\left\{\mathbb{Z}^{+}, Y, d, \mathfrak{s}\right\}$ associated with the HJLS specified by (34)-(35), where $\mathfrak{s} \subset \mathfrak{S}$ is the collection of stochastic motions with initial state $y_{0}=\left[\begin{array}{ll}x_{0}^{\mathrm{T}} & e_{1}^{\mathrm{T}}\end{array}\right]^{\mathrm{T}}$, and $x_{0} \in \mathbb{R}^{n}$ with $\left\|x_{0}\right\|<\alpha$. Let $\bar{\sigma}\left(A_{0}\right)<1$, $\sigma\left(A_{1}\right)>1$ and $\sigma\left(A_{0}\right) \underline{g}\left(A_{1}\right)>1$. The HJLS is finite-time stable and mean square finite-time stable with respect to $\left(\alpha, \beta, T^{*}-1\right)$, where

$$
T^{*}=\left\lceil 1+\frac{\log \left(\beta / \bar{\sigma}\left(A_{0}\right)\left\|x_{0}\right\|\right)}{\log \left(\bar{\sigma}\left(A_{1}\right)\right)}\right\rceil .
$$

Proof: First, observe that the maximum value that the right hand side of (39) can take on at time $k$ is $\bar{\sigma}\left(A_{1}\right)^{k-1} \bar{\sigma}\left(A_{0}\right)\left\|x_{0}\right\|$. Since $\bar{\sigma}\left(A_{0}\right)\left\|x_{0}\right\|<\alpha$, the shortest time at which the right hand side (39) can reach or exceed $\beta$ is $k=T^{*}$. Hence, $\max _{\omega \in \Omega}\{\|\boldsymbol{x}(k, \omega)\|\}<\beta$ for all $k \in \mathcal{I}_{T^{*}}=\left\{0,1, \ldots, T^{*}-1\right\}$. Also, note that $\mathbf{E}\left\{\|\boldsymbol{x}(k)\|^{2}\right\} \leq \max _{\omega \in \Omega}\left\{\|\boldsymbol{x}(k, \omega)\|^{2}\right\}<\beta^{2}$ for all $k \in \mathcal{I}_{T^{*}}$. These two facts directly prove that the HJLS is finite-time stable and mean square finite-time stable with respect to $\left(\alpha, \beta, T^{*}-1\right)$.

If $\beta=\alpha$ then Lemma III.4.3 shows that the performance boundary will not be reached for at least $T^{*}-1$ samples. During this time, the output of the analog to signal converter is zero, and the finite state machine evolution is simply described by $\boldsymbol{\theta}(k)=\boldsymbol{N}(k-1)$ for $k \leq T^{*}$. If, in addition, the JLS is scalar then $T^{*}$ also represents 
the maximum time over which all sample solutions behave as a Markov jump linear system (see Lemma III.4.1).

The results introduced in this section are based on bounding the norm of the matrices $A_{i}$ with their maximum and minimum singular values. It is well-known that these bounds are generally not tight. The following section introduces two less conservative results that are not based on these bounds. Instead, they utilize a lifting technique employed successfully, for example, by (Costa \& Fragoso 1993) to study Markov jump linear systems. A disadvantage of this approach, however, is a sharp increase in the dimensionality of the matrices involved.

\section{III.5 STABILITY ANALYSIS OF HJLS'S USING A LIFTING TECHNIQUE}

It was shown by (Costa \& Fragoso 1993) that the limit behavior of the second moment of a Markov jump linear system, $\mathbf{E}\left\{\|\boldsymbol{x}(k)\|^{2}\right\}$, is related to the asymptotic stability of a discrete-time deterministic LTI system of the form

$$
\tilde{q}(k+1)=\mathcal{A} \tilde{q}(k)
$$

where the lifted matrix, $\mathcal{A}$, is formed by taking into account the MJLS's state matrices and the properties of the Markov chain driving the MJLS. Moreover, it was shown that for a MJLS $\mathbf{E}\left\{\|\boldsymbol{x}(k)\|^{2}\right\} \rightarrow 0$ as $k \rightarrow \infty$ if and only if $\rho(\mathcal{A})<1$. In this section, this lifting technique adapted to derive two sufficient MS stability conditions for two different subclasses of HJLS's. Subsection III.5.1 introduces a sufficient mean square stability test valid for any HJLS with a performance map. The sufficiency test consists of checking whether the spectral radius of a particular $\mathcal{A}$ matrix is less than 
1. Although the test is derived by carefully exploiting the unique structure of HJLS's with performance map, it is possible to extend this test to other types of HJLS's via a more complex two step approach. Subsection III.5.2 presents the first step of this extension. It introduces a new sufficient mean square stability condition that applies to any jump linear system driven by a general discrete-time, finite-state stochastic process $\{\boldsymbol{\theta}(k), k \geq 0\}$. The second step of the extension requires one to compute a matrix of parameters, $M_{\theta}$, which depends on the specific structure of the HJLS under consideration. The discussion of the general method for computing $M_{\theta}$ is delayed until Chapter IV because it requires the study of HJLS's from the point of view of Markov chains. Nevertheless, the method is illustrated for two particular examples at the end of the section.

\section{III.5.1 Mean Square Stability of HJLS's with Performance Map}

This section presents a practical mean square stability test for the subclass of HJLS with performance map. The test makes use of the following supporting results.

Lemma III.5.1 Consider the space $L^{2}(\Omega, \mathscr{F}, \operatorname{Pr})$ of square integrable random variables. For any $\boldsymbol{x}, \boldsymbol{y} \in L^{2}(\Omega, \mathscr{F}, \operatorname{Pr})$ it follows that $\max \left\{|\mathbf{E}\{\boldsymbol{x} \boldsymbol{y}\}|, \mathbf{E}\left\{\boldsymbol{x}^{2}\right\}, \mathbf{E}\left\{\boldsymbol{y}^{2}\right\}\right\}=$ $\max \left\{\mathbf{E}\left\{\boldsymbol{x}^{2}\right\}, \mathbf{E}\left\{\boldsymbol{y}^{2}\right\}\right\}$

Proof: Suppose $|\mathbf{E}\{\boldsymbol{x} \boldsymbol{y}\}|>\mathbf{E}\left\{\boldsymbol{x}^{2}\right\}$ and $|\mathbf{E}\{\boldsymbol{x} \boldsymbol{y}\}|^{2}>\mathbf{E}\left\{\boldsymbol{y}^{2}\right\}$. Then $|\mathbf{E}\{\boldsymbol{x} \boldsymbol{y}\}|>$ $\mathbf{E}\left\{\boldsymbol{x}^{2}\right\} \mathbf{E}\left\{\boldsymbol{x}^{2}\right\}$, which contradicts the Schwarz's inequality. Thus either $|\mathbf{E}\{\boldsymbol{x} \boldsymbol{y}\}| \leq$ $\mathbf{E}\left\{\boldsymbol{x}^{2}\right\}$ or $|\mathbf{E}\{\boldsymbol{x} \boldsymbol{y}\}| \leq \mathbf{E}\left\{\boldsymbol{y}^{2}\right\}$, or both inequalities are satisfied. Hence, $\max \left\{|\mathbf{E}\{\boldsymbol{x} \boldsymbol{y}\}|, \mathbf{E}\left\{\boldsymbol{x}^{2}\right\}, \mathbf{E}\left\{\boldsymbol{y}^{2}\right\}\right\}=\max \left\{\mathbf{E}\left\{\boldsymbol{x}^{2}\right\}, \mathbf{E}\left\{\boldsymbol{y}^{2}\right\}\right\}$ 
Lemma III.5.2 Let $\boldsymbol{X} \in \mathbb{R}^{n}$ be a random vector such that $\mathbf{E}\left\{\boldsymbol{X}^{\mathrm{T}} \boldsymbol{X}\right\}<\infty$. Then

$$
\left\|\operatorname{vec}\left(\mathbf{E}\left\{\boldsymbol{X} \boldsymbol{X}^{\mathrm{T}}\right\}\right)\right\|_{\infty}=\max _{i \in\{1, \ldots, n\}}\left\{\mathbf{E}\left\{\boldsymbol{x}_{i}^{2}\right\}\right\}
$$

where $\boldsymbol{x}_{\boldsymbol{i}}$ is the $i$-th entry of $\boldsymbol{X}$, and $\operatorname{vec}(\cdot)$ is the column stacking operator.

Proof: Since $\mathbf{E}\left\{\boldsymbol{X}^{\mathrm{T}} \boldsymbol{X}\right\}<\infty$ then $\boldsymbol{x}_{i} \in L^{2}(\Omega, \mathscr{F}, \operatorname{Pr})$, for all $i \in\{1, \ldots, n\}$. It follows from Lemma III.5.1that

$$
\max \left\{\left|\mathbf{E}\left\{\boldsymbol{x}_{i} \boldsymbol{x}_{j}\right\}\right|, \mathbf{E}\left\{\boldsymbol{x}_{i}^{2}\right\}, \mathbf{E}\left\{\boldsymbol{x}_{j}^{2}\right\}\right\}=\max \left\{\mathbf{E}\left\{\boldsymbol{x}_{i}^{2}\right\}, \mathbf{E}\left\{\boldsymbol{x}_{j}^{2}\right\}\right\}
$$

for every $i, j \in\{1, \ldots, n\}$. Therefore

$$
\left\|\operatorname{vec}\left(\mathbf{E}\left\{\boldsymbol{X} \boldsymbol{X}^{\mathrm{T}}\right\}\right)\right\|_{\infty}=\max _{i, j \in\{1, \ldots, n\}}\left\{\left|\mathbf{E}\left\{\boldsymbol{x}_{i} \boldsymbol{x}_{j}\right\}\right|\right\}=\max _{i \in\{1, \ldots, n\}}\left\{\mathbf{E}\left\{\boldsymbol{x}_{i}^{2}\right\}\right\}
$$

The main result of this section follows.

Theorem III.5.1 Consider the stochastic dynamical system $\left\{\mathbb{Z}^{+}, Y, d, \mathfrak{S}\right\}$ associated with the following hybrid jump linear system with performance map (see Definition II.3.2)

$$
\begin{aligned}
\boldsymbol{x}(k+1) & =A_{\boldsymbol{\theta}(k)} \boldsymbol{x}(k) \\
\boldsymbol{\nu}(k) & =\sum_{i=0}^{\ell_{\nu}-1} i 1_{\left\{\alpha_{i} \leq\|\boldsymbol{x}(k)\|<\alpha_{i+1}\right\}}, \\
\boldsymbol{\theta}(k) & =\ell_{\varphi} \boldsymbol{\nu}(k)+\boldsymbol{\varphi}(k),
\end{aligned}
$$

where $\boldsymbol{x}(k) \in \mathbb{R}^{n}, 0=\alpha_{0}<\alpha_{1}<\cdots<\alpha_{\ell_{\nu}}=\infty$, and $\{\varphi(k), k \geq 0\}$ is a Markov chain with $\varphi(k) \in \mathcal{I}_{\ell_{\varphi}}$, transition probability matrix $\Pi_{\varphi}=\left[p_{i, j}^{\varphi}\right]$ and initial distribution $\mu_{0}^{\varphi}$. Let $\mathcal{A}$ be defined as

$$
\mathcal{A} \triangleq\left(\Pi_{\theta}^{\mathrm{T}} \otimes I_{n^{2}}\right) \operatorname{diag}\left(A_{0} \otimes A_{0}, \ldots, A_{\ell_{\nu} \ell_{\varphi}-1} \otimes A_{\ell_{\nu} \ell_{\varphi}-1}\right)
$$

where $\Pi_{\theta}=E_{\ell_{\nu}} \otimes \Pi_{\varphi}$. If $\|\mathcal{A}\|_{\infty}<1$ then the HJLS in (44) is mean square stable. 
Proof : Fix any stochastic motion in $\mathfrak{S}$ (that is, choose any initial state $y_{0}=\left[\begin{array}{ll}x_{0}^{\mathrm{T}} & z_{0}^{\mathrm{T}}\end{array}\right]$ and initial distribution $\left.\mu_{0}^{\varphi}\right)$. Let $Q(k+1)=\mathbf{E}\left\{\boldsymbol{x}(k+1) \boldsymbol{x}^{\mathrm{T}}(k+1)\right\}$ and define

$$
Q_{i}(k+1) \triangleq \mathbf{E}\left\{\boldsymbol{x}(k+1) \boldsymbol{x}^{\mathbf{T}}(k+1) \mathbf{1}_{\{\boldsymbol{\theta}(k+1)=i\}}\right\}
$$

so that $Q(k+1)=\sum_{i=0}^{\ell_{\nu} \ell_{\varphi}-1} Q_{i}(k+1)$. Then it follows that

$$
\begin{aligned}
Q_{i}(k+1) & =\mathbf{E}\left\{A_{\boldsymbol{\theta}(k)} \boldsymbol{x}(k) \boldsymbol{x}^{\mathrm{T}}(k) A_{\boldsymbol{\theta}(k)}^{\mathrm{T}} \mathbf{1}_{\{\boldsymbol{\theta}(k+1)=i\}}\right\} \\
& =\sum_{j=0}^{\ell_{\nu} \ell_{\varphi}-1} \mathbf{E}\left\{A_{j} \boldsymbol{x}(k) \boldsymbol{x}^{\mathrm{T}}(k) A_{j}^{\mathrm{T}} \mathbf{1}_{\{\boldsymbol{\theta}(k+1)=i, \boldsymbol{\theta}(k)=j\}}\right\} \\
& =\sum_{j=0}^{\ell_{\nu} \ell_{\varphi}-1} A_{j} \mathbf{E}\left\{\boldsymbol{x}(k) \boldsymbol{x}^{\mathrm{T}}(k) \mathbf{1}_{\{\boldsymbol{\theta}(k+1)=i, \boldsymbol{\theta}(k)=j\}}\right\} A_{j}^{\mathrm{T}} .
\end{aligned}
$$

Now define $\boldsymbol{q}_{\boldsymbol{x}}(k) \triangleq \operatorname{vec}\left(\boldsymbol{x}(k) \boldsymbol{x}^{\mathrm{T}}(k)\right), \tilde{q}_{i}(k+1) \triangleq \operatorname{vec}\left(Q_{i}(k+1)\right)=\mathbf{E}\left\{\boldsymbol{q}_{\boldsymbol{x}}(k+\right.$ 1) $\left.1_{\{\boldsymbol{\theta}(k+1)=i\}}\right\}$ and apply the $\operatorname{vec}(\cdot)$ operator to the equation above to obtain

$$
\tilde{q}_{i}(k+1)=\sum_{j=0}^{\ell_{\nu} \ell_{\varphi}-1}\left(A_{j} \otimes A_{j}\right) \mathbf{E}\left\{\boldsymbol{q}_{x}(k) \mathbf{1}_{\{\boldsymbol{\theta}(k+1)=i, \boldsymbol{\theta}(k)=j\}}\right\} .
$$

As shown in Lemma A.3.1, if a random variable $\boldsymbol{\xi}$ is measurable with respect to a $\sigma$-algebra $\mathcal{G} \subset \mathscr{F}$ then $\boldsymbol{\xi}=\mathbf{E}\{\boldsymbol{\xi} \mid \mathcal{G}\}$ and $\mathbf{E}\{\boldsymbol{\xi}\}=\mathbf{E}\{\mathbf{E}\{\boldsymbol{\xi} \mid \mathcal{G}\}\}$. Let $\mathscr{F}_{k} \triangleq$ $\sigma(\boldsymbol{\theta}(0), \ldots, \boldsymbol{\theta}(k))$ and observe that

$$
\begin{aligned}
\mathbf{E}\left\{\boldsymbol{q}_{\boldsymbol{x}}(k) \mathbf{1}_{\{\boldsymbol{\theta}(k+1)=i, \boldsymbol{\theta}(k)=j\}}\right\} & =\mathbf{E}\left\{\mathbf{E}\left\{\boldsymbol{q}_{x}(k) \mathbf{1}_{\{\boldsymbol{\theta}(k+1)=i, \boldsymbol{\theta}(k)=j\}} \mid \mathscr{F}_{k}\right\}\right\} \\
& =\mathbf{E}\left\{\boldsymbol{q}_{x}(k) \operatorname{Pr}\left\{\boldsymbol{\theta}(k+1)=i, \boldsymbol{\theta}(k)=j \mid \mathscr{F}_{k}\right\}\right\} \\
& =\mathbf{E}\left\{\boldsymbol{q}_{x}(k) \operatorname{Pr}\left\{\boldsymbol{\theta}(k+1)=i \mid \mathscr{F}_{k}\right\} \mathbf{1}_{\{\boldsymbol{\theta}(k)=j\}}\right\},
\end{aligned}
$$

where the last equality follows from the next set of identities.

$$
\begin{aligned}
& \operatorname{Pr}\left\{\boldsymbol{\theta}(k+1)=i, \boldsymbol{\theta}(k)=j \mid \mathscr{F}_{k}\right\} \\
& \quad=\sum_{l_{k}, \ldots, l_{0}=0}^{\ell_{\nu} \ell_{\varphi}-1} \operatorname{Pr}\left\{\boldsymbol{\theta}(k+1)=i, \boldsymbol{\theta}(k)=j \mid \boldsymbol{\theta}(k)=l_{k}, \ldots, \boldsymbol{\theta}(0)=l_{0}\right\} 1_{\left\{\boldsymbol{\theta}(k)=l_{k}, \ldots, \boldsymbol{\theta}(0)=l_{0}\right\}}
\end{aligned}
$$




$$
\begin{aligned}
& =\sum_{l_{k}, \ldots, l_{0}=0}^{\ell_{\nu} \ell_{\varphi}-1} \operatorname{Pr}\left\{\boldsymbol{\theta}(k+1)=i \mid \boldsymbol{\theta}(k)=j, \ldots, \boldsymbol{\theta}(0)=l_{0}\right\} 1_{\left\{\boldsymbol{\theta}(k)=l_{k}, \ldots, \boldsymbol{\theta}(0)=l_{0}\right\}} \mathbf{1}_{\{\boldsymbol{\theta}(k)=j\}} \\
& =\operatorname{Pr}\left\{\boldsymbol{\theta}(k+1)=i \mid \mathscr{F}_{k}\right\} \mathbf{1}_{\{\boldsymbol{\theta}(k)=j\}}
\end{aligned}
$$

Note that the value of (48) changes depending on which of the following four mutually exclusive conditions is satisfied:

(i) The set $\{\boldsymbol{\theta}(k)=j\}$ is empty.

(ii) The set $\{\boldsymbol{\theta}(k)=j\}$ is not empty, but there are no non-empty sets of the form $\left\{\boldsymbol{\theta}(k+1)=i, \boldsymbol{\theta}(k)=j, \boldsymbol{\theta}(k-1)=l_{k-1}, \ldots, \boldsymbol{\theta}(0)=l_{0}\right\}$.

(iii) The set $\{\boldsymbol{\theta}(k)=j\}$ and some or all of the sets $\{\boldsymbol{\theta}(k+1)=i, \boldsymbol{\theta}(k)=$ $\left.j, \ldots, \boldsymbol{\theta}(0)=l_{0}\right\}$ are not empty, but $p_{\hat{\jmath}, \hat{\imath}}^{\varphi}=\operatorname{Pr}\{\varphi(k+1)=\hat{\imath} \mid \varphi(k)=\hat{\jmath}\}=0$, where $\hat{\jmath}=j-\ell_{\varphi}\left\lfloor j / \ell_{\varphi}\right\rfloor, \hat{\imath}=i-\ell_{\varphi}\left\lfloor i / \ell_{\varphi}\right\rfloor(\lfloor i\rfloor$ returns the maximum integer smaller than or equal to $i$ ).

(iv) The set $\{\boldsymbol{\theta}(k)=j\}$ and some or all of the sets $\{\boldsymbol{\theta}(k+1)=i, \boldsymbol{\theta}(k)=$ $\left.j, \ldots, \boldsymbol{\theta}(0)=l_{0}\right\}$ are not empty, and $p_{j, \imath}^{\varphi}>0$.

In (iii) observe that $\{\boldsymbol{\theta}(k)=j\}=\left\{\boldsymbol{\varphi}(k)=\hat{\jmath}, \boldsymbol{\nu}(k)=\left\lfloor j / \ell_{\varphi}\right\rfloor\right\}$. So a very simple argument shows that $\left\{\boldsymbol{\theta}(k+1)=i, \boldsymbol{\theta}(k)=j, \ldots, \boldsymbol{\theta}(0)=l_{0}\right\}=\{\boldsymbol{\varphi}(k+1)=\hat{\imath}, \boldsymbol{\varphi}(k)=$ $\left.\hat{\jmath}, \ldots, \varphi(0)=\hat{l}_{0}\right\}$. Thus,

$$
\begin{aligned}
\operatorname{Pr}\{\boldsymbol{\theta}(k+1)=i \mid \boldsymbol{\theta}(k)=j, & \left.\ldots, \boldsymbol{\theta}(0)=l_{0}\right\} \\
& =\operatorname{Pr}\left\{\varphi(k+1)=\hat{\imath} \mid \varphi(k)=\hat{\jmath}, \ldots, \varphi(0)=\hat{l}_{0}\right\} \\
& =p_{\hat{\jmath}, \hat{\imath}}^{\varphi} \\
& =0 .
\end{aligned}
$$

Note that (47) equals zero according to (i)-(iii) since the random variable $\operatorname{Pr}\{\boldsymbol{\theta}(k+$ 1) $\left.=i \mid \mathscr{F}_{k}\right\} 1_{\{\theta(k)=j\}}$ is a constant equal to zero. This is not the case for the last 
condition from which it follows that $\operatorname{Pr}\left\{\boldsymbol{\theta}(k+1)=i \mid \mathscr{F}_{k}\right\} \mathbf{1}_{\{\boldsymbol{\theta}(k)=j\}} \in\left\{0, p_{\hat{j}, \hat{\imath}}^{\varphi}\right\}$. Thus, Lemma III.5.2 implies that

$$
\begin{aligned}
\left\|\mathbf{E}\left\{\boldsymbol{q}_{x}(k) \mathbf{1}_{\{\boldsymbol{\theta}(k+1)=i, \boldsymbol{\theta}(k)=j\}}\right\}\right\|_{\infty} & =\max _{m}\left\{\mathbf{E}\left\{\boldsymbol{x}_{m}^{2} \operatorname{Pr}\left\{\boldsymbol{\theta}(k+1)=i \mid \mathscr{F}_{k}\right\}\right\} \mathbf{1}_{\{\boldsymbol{\theta}(k)=j\}}\right\} \\
& \leq \max _{m}\left\{\mathbf{E}\left\{\boldsymbol{x}_{m}^{2} p_{j, \hat{\imath}}^{\varphi} \mathbf{1}_{\{\boldsymbol{\theta}(k)=j\}}\right\}\right\} \\
& \leq p_{\hat{\jmath}, \hat{i}}^{\varphi}\left\|\mathbf{E}\left\{\boldsymbol{q}_{x}(k) \mathbf{1}_{\{\boldsymbol{\theta}(k)=j\}}\right\}\right\|_{\infty} \\
& \leq p_{\hat{j}, \hat{i}}^{\varphi}\left\|\tilde{q}_{j}(k)\right\|_{\infty} .
\end{aligned}
$$

Now, define $h_{i} \triangleq\left[p_{0, \hat{\imath}}^{\varphi}\left(A_{0} \otimes A_{0}\right), \ldots, p_{\left(\ell_{\varphi}-1\right), \hat{\imath}}^{\varphi}\left(A_{\ell_{\nu} \ell_{\varphi}-1} \otimes A_{\ell_{\nu} \ell_{\varphi}-1}\right)\right], \quad \tilde{q}(k) \triangleq$ $\left[\tilde{q}_{0}(k)^{\mathrm{T}}, \ldots, \tilde{q}_{\ell_{\nu} \ell_{\varphi}-1}(k)^{\mathrm{T}}\right]^{\mathrm{T}}$, and let $\mathcal{I}(k) \subseteq \mathcal{I}_{\ell_{\nu} \ell_{\varphi}}$ be the set of indices for which (48) falls under condition (iv). Then it follows from (i)-(iv), (46), and Lemma III.5.2 that

$$
\begin{aligned}
\left\|\tilde{q}_{i}(k+1)\right\|_{\infty} & \leq\left\|h_{i}\right\|_{\infty} \max _{m \in \mathcal{I}(k)}\left\{\left\|\frac{\mathbf{E}\left\{\boldsymbol{q}_{x}(k) \mathbf{1}_{\{\boldsymbol{\theta}(k+1)=i, \boldsymbol{\theta}(k)=m\}}\right\}}{p_{\hat{m}, \hat{\imath}}}\right\|_{\infty}\right\} \\
& \leq\left\|h_{i}\right\|_{\infty} \max _{m \in \mathcal{I}(k)}\left\{\left\|\tilde{q}_{m}(k)\right\|_{\infty}\right\} \\
& \leq\left\|h_{i}\right\|_{\infty} \max _{0 \leq m \leq \ell_{\nu} \ell_{\varphi}-1}\left\{\left\|\tilde{q}_{m}(k)\right\|_{\infty}\right\} \\
& \leq\left\|h_{i}\right\|_{\infty}\|\tilde{q}(k)\|_{\infty} .
\end{aligned}
$$

Note that if $m=j+\ell_{\varphi}$ then $\hat{m}=\hat{\jmath}$, so $p_{\hat{m}, \hat{\imath}}^{\varphi}=p_{\hat{\jmath}, \hat{\imath}}^{\varphi}$. Also, $\|\mathcal{A}\|_{\infty}=\max _{i}\left\{\|\left[p_{0, \hat{\imath}}^{\varphi}\left(A_{0} \otimes\right.\right.\right.$ $\left.\left.\left.A_{0}\right), \ldots, p_{\left(\ell_{\varphi}-1\right), \hat{\imath}}^{\varphi}\left(A_{\ell_{\nu} \ell_{\varphi}-1} \otimes A_{\ell_{\nu} \ell_{\varphi}-1}\right)\right] \|_{\infty}\right\}=\max _{i}\left\{\left\|h_{i}\right\|_{\infty}\right\}$. Thus,

$$
\begin{aligned}
\|\tilde{q}(k+1)\|_{\infty} & =\max _{0 \leq i \leq \ell_{\nu} \ell_{\varphi}-1}\left\{\left\|\tilde{q}_{i}(k+1)\right\|_{\infty}\right\} \\
& \leq \max _{0 \leq i \leq \ell_{\nu} \ell_{\varphi}-1}\left\{\left\|h_{i}\right\|_{\infty}\right\}\|\tilde{q}(k)\|_{\infty} \leq\|\mathcal{A}\|_{\infty}\|\tilde{q}(k)\|_{\infty}
\end{aligned}
$$

Clearly, if $\|\mathcal{A}\|_{\infty}<1$ then $\tilde{q}(k+1) \rightarrow 0$ as $k \rightarrow \infty$, which in turn implies that $Q(k+1) \rightarrow 0$ as $k \rightarrow \infty$. The same conclusion can be reached for any other stochastic 
motion in $\mathfrak{S}$. Thus, the HJLS in (44) is mean square stable.

As a final remark, define, for every $j \in \mathcal{I}_{\ell_{\nu}}$, the matrices

$$
\mathcal{A}_{j}=\left(\Pi_{\varphi}^{\mathrm{T}} \otimes I_{n^{2}}\right) \operatorname{diag}\left(A_{j \ell_{\varphi}} \otimes A_{j \ell_{\varphi}}, A_{j \ell_{\varphi}+1} \otimes A_{j \ell_{\varphi}+1}, \ldots, A_{j \ell_{\varphi}+\ell_{\varphi}-1} \otimes A_{j \ell_{\varphi}+\ell_{\varphi}-1}\right)
$$

and observe that

$$
\mathcal{A}=\left[\begin{array}{ccc}
\mathcal{A}_{0} & \cdots & \mathcal{A}_{\ell_{\nu}-1} \\
\vdots & \ddots & \vdots \\
\mathcal{A}_{0} & \cdots & \mathcal{A}_{\ell_{\nu}-1}
\end{array}\right]
$$

Thus, Theorem III.5.1 can be expressed as follows.

Corollary III.5.1 If $\left\|\left[\mathcal{A}_{0} \cdots \mathcal{A}_{\ell_{\nu}-1}\right]\right\|_{\infty}<1$ then system (44) is mean square stable.

\section{III.5.2 Mean Square Stability of JLS's Driven by General Discrete-Time, Finite-State Stochastic Processes}

Consider the stochastic dynamical system $\left\{\mathbb{Z}^{+}, Y, d, \mathfrak{S}\right\}$ associated with a HJLS defined as in (8). In particular, recall that its associated jump linear closed-loop system is given by

$$
\boldsymbol{x}(k+1)=A_{\boldsymbol{\theta}(k)} \boldsymbol{x}(k),
$$

where $\{\boldsymbol{\theta}(k), k \geq 0\}$ is, usually, not a Markov chain but a general discrete-time stochastic process that takes values from the set $\mathcal{I}_{\ell_{O}}$. The main result in this subsection, Theorem III.5.2, provides a new sufficient mean square stability condition for any jump linear system such as (49), using a lifting technique similar to the one employed in Theorem III.5.1. Since $\{\boldsymbol{\theta}(k), k \geq 0\}$ is a general discrete-time stochastic process, the result in Theorem III.5.2 depends on estimated bounds for the "transition 
probabilities" of $\{\boldsymbol{\theta}(k), k \geq 0\}$. These bounds must be derived for each particular HJLS under consideration. This derivation, however, is relegated to Chapter IV (see Theorem IV.4.4).

Recall that $\mathscr{F}_{k}=\sigma(\boldsymbol{\theta}(0), \ldots, \boldsymbol{\theta}(k))$ and that both $\boldsymbol{\theta}(k)$ and $\boldsymbol{x}(k)$ are $\mathscr{F}_{k^{-}}$ measurable random vectors. The stability condition in Theorem III.5.2 relies on computing

$$
\begin{aligned}
& \mathbf{E}\left\{\mathbf{1}_{\{\boldsymbol{\theta}(k+1)=j\}} \mid \mathscr{F}_{k}\right\} \mathbf{1}_{\{\boldsymbol{\theta}(k)=i\}}= \\
& \sum_{l_{0}=0}^{\ell_{O}-1} \cdots \sum_{l_{k-1}=0}^{\ell_{O}-1} \operatorname{Pr}\left\{\boldsymbol{\theta}(k+1)=j \mid \boldsymbol{\theta}(k)=i, \boldsymbol{\theta}(k-1)=l_{k-1}, \ldots, \boldsymbol{\theta}(0)=l_{0}\right\} \\
& \mathbf{1}_{\left\{\boldsymbol{\theta}(k)=i, \boldsymbol{\theta}(k-1)=l_{k-1}, \ldots, \boldsymbol{\theta}(0)=l_{0}\right\}}
\end{aligned}
$$

where $l_{0}, \ldots, l_{k-1} \in \mathcal{I}_{\ell_{O}}$. It follows from the definition that $\mathbf{E}\left\{\mathbf{1}_{\{\boldsymbol{\theta}(k+1)=j\}} \mid \mathscr{F}_{k}\right\} \mathbf{1}_{\{\boldsymbol{\theta}(k)=i\}}$ is a bounded random variable that takes values in $[0,1]$. Thus, there always exists constants $0 \leq M_{i, j}(k) \leq 1$ such that

$$
\mathbf{E}\left\{\mathbf{1}_{\{\boldsymbol{\theta}(k+1)=j\}} \mid \mathscr{F}_{k}\right\} \mathbf{1}_{\{\boldsymbol{\theta}(k)=i\}} \leq M_{i, j}(k) 1_{\{\boldsymbol{\theta}(k)=i\}},
$$

for every $k \geq 0$. Clearly, the set $\left\{M_{i, j}(0), M_{i, j}(1), \ldots\right\}$ is bounded from above, so there exists constants $M_{i, j}$ such that $M_{i, j} \geq M_{i, j}(k)$ for all $k \geq 0$. This motivates the following definition.

Definition III.5.1 $A$ matrix $M_{\theta} \in \mathbb{R}^{\ell_{O} \times \ell_{O}}$ is an upper bound matrix with respect to the random variables defined in (50), or simply $M_{\theta}$ is an upper bound matrix, if each of its entries, $M_{i, j}{ }^{1}$ satisfies the condition

$$
\mathbf{E}\left\{\mathbf{1}_{\{\boldsymbol{\theta}(k+1)=j\}} \mid \mathscr{F}_{k}\right\} \mathbf{1}_{\{\boldsymbol{\theta}(k)=i\}} \leq M_{i, j} \mathbf{1}_{\{\theta(k)=i\}},
$$

\footnotetext{
${ }^{1}$ Note that the rows and columns of $M_{\theta}$ are labeled from 0 to $\ell_{O}-1$.
} 
for every $k \geq 0$, where $i, j \in \mathcal{I}_{\ell_{O}}$.

The following is the main result of this section.

Theorem III.5.2 Consider the stochastic dynamical system system $\left\{\mathbb{Z}^{+}, \mathbb{R}^{n},\|\cdot\|, \mathfrak{S}\right\}$ associated with the jump linear system ${ }^{2}$

$$
\boldsymbol{x}(k+1)=A_{\boldsymbol{\theta}(k)} \boldsymbol{x}(k), \quad \boldsymbol{x}(0)=\boldsymbol{x}_{0},
$$

where $\{\boldsymbol{\theta}(k), k \geq 0\}$ is any discrete-time stochastic process independent of $\boldsymbol{x}_{0}$, such that $\boldsymbol{\theta}(k) \in \mathcal{I}_{\ell_{O}}$, for every $k \geq 0$. Let $M_{\theta}=\left[M_{i, j}\right]$ be an upper bound matrix for the random variables in (50) and define

$$
\mathcal{A}_{M_{\theta}} \triangleq\left(M_{\theta}^{\mathrm{T}} \otimes I_{n^{2}}\right) \operatorname{diag}\left(A_{0} \otimes A_{0}, \ldots, A_{\ell_{O}-1} \otimes A_{\ell_{O}-1}\right)
$$

If $\rho\left(\mathcal{A}_{M_{\theta}}\right)<1$, then $(51)$ is second moment stable.

Remark III.5.1 Note that the stochastic dynamical system $\left\{\mathbb{Z}^{+}, \mathbb{R}^{n},\|\cdot\|, \mathfrak{S}\right\}$ is associated with the jump linear system in (51) only. No relationship with any underlying HJLS is presumed or needed to prove this result. This is important to emphasize because Theorem III.5.2 can be applied outside the context of HJLS's. Its application to HJLS's, however, is fully explored in Chapter IV.

Proof: The proof parallels that of (Theorem 2.1, Ji \& Chizeck 1990b). First, fix any stochastic motion in $\mathfrak{S}$ and observe that $\rho\left(\mathcal{A}_{M_{\theta}}\right)<1$ implies that for any given set of real, symmetric, positive definite matrices $\left\{W_{i}>0, i \in \mathcal{I}_{\ell_{0}}\right\}$ there exists a set of real, symmetric, positive definite matrices $\left\{P_{i}>0, i \in \mathcal{I}_{\ell_{0}}\right\}$ such that

$$
\sum_{j=0}^{\ell_{O}-1} M_{i, j} A_{i}^{\mathrm{T}} P_{j} A_{i}-P_{i}=-W_{i}
$$

\footnotetext{
${ }^{2}$ This theorem was developed in collaboration with Mr. Heber Herencia-Zapana (Tejada, González \& Gray 2007).
} 
holds for every $i \in \mathcal{I}_{\ell_{O}}$ (Costa \& Fragoso 1993). Next, consider the Lyapunov function $\boldsymbol{V}(k)=\boldsymbol{x}^{\mathrm{T}}(k) P_{\boldsymbol{\theta}(k)} \boldsymbol{x}(k)$, and observe that

$$
\underline{\lambda}_{P}\|\boldsymbol{x}(k)\|^{2} \leq \boldsymbol{V}(k) \leq \bar{\lambda}_{P}\|\boldsymbol{x}(k)\|^{2}
$$

where $\underline{\lambda}_{P}$ and $\bar{\lambda}_{P}$ are, respectively, the smallest and largest eigenvalues of $\left\{P_{i}, i \in\right.$ $\left.\mathcal{I}_{\ell_{O}}\right\}$. Fix an initial state $x_{0}$, let $\mathscr{F}_{k}=\sigma(\boldsymbol{\theta}(0), \ldots, \boldsymbol{\theta}(k))$ and note that

$$
\begin{aligned}
\mathbf{E}\left\{\boldsymbol{V}(k+1) \mid \mathscr{F}_{k}, x_{0}\right\} & =\mathbf{E}\left\{\boldsymbol{x}^{\mathrm{T}}(k) A_{\boldsymbol{\theta}(k)}^{\mathrm{T}} P_{\boldsymbol{\theta}(k+1)} A_{\boldsymbol{\theta}(k)} \boldsymbol{x}(k) \mid \mathscr{F}_{k}, x_{0}\right\} \\
& =\sum_{j=0}^{\ell_{O}-1} \mathbf{E}\left\{\boldsymbol{x}^{\mathrm{T}}(k) A_{\boldsymbol{\theta}(k)}^{\mathrm{T}} P_{j} \mathbf{1}_{\{\boldsymbol{\theta}(k+1)=j\}} A_{\boldsymbol{\theta}(k)} \boldsymbol{x}(k) \mid \mathscr{F}_{k}, x_{0}\right\} .
\end{aligned}
$$

Note that for a given $x_{0}$, both $\boldsymbol{x}(k)$ and $\boldsymbol{\theta}(k)$ are measurable with respect to $\mathscr{F}_{k}$. Thus, each term in the sum above can be expanded as follows

$$
\begin{aligned}
\mathbf{E}\left\{\boldsymbol{x}^{\mathrm{T}}(k) A_{\boldsymbol{\theta}(k)}^{\mathrm{T}}\right. & \left.P_{j} \mathbf{1}_{\{\boldsymbol{\theta}(k+1)=j\}} A_{\boldsymbol{\theta}(k)} \boldsymbol{x}(k) \mid \mathscr{F}_{k}, x_{0}\right\} \\
& =\sum_{i=0}^{\ell_{O}-\mathbf{1}} \mathbf{E}\left\{\boldsymbol{x}^{\mathrm{T}}(k) A_{i}^{\mathrm{T}} P_{j} \mathbf{1}_{\{\boldsymbol{\theta}(k+1)=j\}} A_{i} \boldsymbol{x}(k) \mathbf{1}_{\{\boldsymbol{\theta}(k)=i\}} \mid \mathscr{F}_{k}, x_{0}\right\} \\
& =\sum_{i=0}^{\ell_{O}-\mathbf{1}} \boldsymbol{x}^{\mathrm{T}}(k) A_{i}^{\mathrm{T}} P_{j} A_{i} \mathbf{E}\left\{\mathbf{1}_{\{\boldsymbol{\theta}(k+1)=j\}} \mid \mathscr{F}_{k}, x_{0}\right\} \boldsymbol{x}(k) \mathbf{1}_{\{\boldsymbol{\theta}(k)=i\}} .
\end{aligned}
$$

Since $P_{j}$ is positive definite, it follows that $\boldsymbol{x}^{\mathrm{T}}(k) A_{i}^{\mathrm{T}} P_{j} A_{i} \boldsymbol{x}(k) \geq 0$. This fact together with the independence of $\boldsymbol{x}_{0}$ and $\{\boldsymbol{\theta}(k), k \geq 0\}$, and the assumption that $M_{\theta}$ is an upper bound matrix, yields

$$
\begin{aligned}
\mathbf{E}\left\{\boldsymbol{x}^{\mathrm{T}}(k) A_{\boldsymbol{\theta}(k)}^{\mathrm{T}} P_{j} \mathbf{1}_{\{\boldsymbol{\theta}(k+1)=j\}} A_{\boldsymbol{\theta}(k)} \boldsymbol{x}(k) \mid \mathscr{F}_{k}, x_{0}\right\} & \\
& \leq \sum_{i=0}^{\ell_{O}-1} \boldsymbol{x}^{\mathrm{T}}(k) A_{i}^{\mathrm{T}} P_{\boldsymbol{j}} A_{\boldsymbol{i}} M_{i, j} \boldsymbol{x}(k) \mathbf{1}_{\{\boldsymbol{\theta}(k)=i\}},
\end{aligned}
$$

and therefore,

$$
\mathbf{E}\left\{\boldsymbol{V}(k+1) \mid \mathscr{F}_{k}, x_{0}\right\} \leq \sum_{i=0}^{\ell_{O}-1} \boldsymbol{x}^{\mathrm{T}}(k)\left[\sum_{j=0}^{\ell_{O}} M_{i, j} A_{i}^{\mathrm{T}} P_{j} A_{i}\right] \boldsymbol{x}(k) \mathbf{1}_{\{\boldsymbol{\theta}(k)=i\}}
$$


Note that $\boldsymbol{V}(k)=\sum_{i=0}^{\ell_{O}-1} \boldsymbol{x}^{\mathrm{T}}(k) P_{i} \boldsymbol{x}(k) \mathbf{1}_{\{\boldsymbol{\theta}(k)=i\}}$, so it follows from the expression above and (52) that

$$
\mathbf{E}\left\{\boldsymbol{V}(k+1) \mid \mathscr{F}_{k}, x_{0}\right\}-\boldsymbol{V}(k) \leq-\boldsymbol{x}^{\mathrm{T}}(k) W_{\boldsymbol{\theta}(k)} \boldsymbol{x}(k)
$$

Next, observe that dividing the expression above by $\boldsymbol{V}(k)$ yields, after some simplifications,

$$
\mathbf{E}\left\{\boldsymbol{V}(k+1) \mid \mathscr{F}_{k}, x_{0}\right\} \leq \alpha \boldsymbol{V}(k)
$$

where $\alpha=1-\left(\underline{\lambda}_{W} / \bar{\lambda}_{P}\right), 0<\alpha<1$, and $\underline{\lambda}_{W}$ is the smallest eigenvalue of $\left\{W_{i}, i \in \mathcal{I}_{\ell_{0}}\right\}$. Since $\mathbf{E}\left\{\mathbf{E}\left\{\boldsymbol{V}(k+1) \mid \mathscr{F}_{k}\right\} \mid \mathscr{F}_{k-1}\right\}=\mathbf{E}\left\{\boldsymbol{V}(k+1) \mid \mathscr{F}_{k-1}\right\}$, it follows from (54) that

$$
\mathbf{E}\left\{\boldsymbol{V}(k+1) \mid \mathscr{F}_{0}, x_{0}\right\}=\mathbf{E}\left\{\boldsymbol{V}(k+1) \mid \boldsymbol{\theta}(0), x_{0}\right\} \leq \alpha^{k+1} \boldsymbol{V}(0)
$$

Finally, taking expectation with respect to $\boldsymbol{\theta}(0)$ in the expression above yields, after using (53),

$$
\begin{aligned}
\mathbf{E}\left\{\|x(k+1)\|^{2} \mid x_{0}\right\} & \leq \frac{\mathbf{E}\left\{\boldsymbol{V}(k+1) \mid x_{0}\right\}}{\underline{\lambda}_{P}} \\
& \leq \frac{\alpha^{k+1} \mathbf{E}\left\{\boldsymbol{V}(0) \mid x_{0}\right\}}{\underline{\lambda}_{P}} \\
& \leq \frac{\bar{\lambda}_{P}}{\underline{\lambda}_{P}} \alpha^{k+1}\left\|x_{0}\right\| .
\end{aligned}
$$

Since this conclusion can be reached for every stochastic motion in $\mathfrak{S}$, it follows from by Definition III.2.7 that (49) is exponentially mean square stable and, consequently, second moment stable.

It is clear from its derivation that the stability test in Theorem III.5.2 is least conservative when the entries of $M_{\theta}$ are as small possible, i.e., when $M_{i, j}=\sup _{k}\left\{M_{i, j}(k)\right\}$. Also, observe that if $\boldsymbol{\theta}(k)$ is a Markov chain, its transition probability matrix, $\Pi_{\theta}$, 
satisfies the upper bound condition in Definition III.5.1, i.e., $M_{\theta}=\Pi_{\theta}$, and Theorem III.5.2 becomes the well-known mean square stability test developed in (Costa \& Fragoso 1993). Finally, note that by letting $M_{i, j}=1, i, j=1, \ldots, \ell_{O}$, Theorem III.5.2 becomes the sufficient condition for asymptotic stability under arbitrary switching introduced in Theorem III.3.2. The rest of this subsection presents two case studies that illustrate the application of Theorem III.5.2.

\section{Case Study 1: Stability of HJLS's with Performance Map}

This case study shows that Theorem III.5.2 can lead to a tighter sufficient mean square stability condition than does Theorem III.5.1.

Theorem III.5.3 Consider the stochastic dynamical system $\left\{\mathbb{Z}^{+}, Y, d, \mathfrak{S}\right\}$ associated with the HJLS with performance map defined in (44). The HJLS is second moment stable whenever $\rho\left(\mathcal{A}_{M_{\theta}}\right)<1$, where

$$
\mathcal{A}_{M_{\theta}}=\left(M_{\theta}^{\mathrm{T}} \otimes I_{n^{2}}\right) \operatorname{diag}\left(A_{0} \otimes A_{0}, \ldots, A_{\ell_{\nu} \ell_{\varphi}-1} \otimes A_{\ell_{\nu} \ell_{\varphi}-1}\right)
$$

Proof: It was shown in the proof of Theorem III.5.1 that for every set of numbers $l_{0}, \ldots, l_{k-1} \in \mathcal{I}_{\ell_{O}}$

$$
\operatorname{Pr}\left\{\boldsymbol{\theta}(k+1)=j \mid \boldsymbol{\theta}(k)=i, \boldsymbol{\theta}(k-1)=l_{k-1}, \ldots, \boldsymbol{\theta}(0)=l_{0}\right\} \in\left\{0, \hat{p}_{\hat{i}, \hat{j}}^{\varphi}\right\}
$$

where $\hat{p}_{\hat{\imath}, \hat{\jmath}}^{\varphi}=\operatorname{Pr}\{\varphi(k+1)=\hat{\jmath} \mid \varphi(k)=\hat{\imath}\}$, and $\hat{\imath}=i-\ell_{\varphi}\left\lfloor i / \ell_{\varphi}\right\rfloor, \hat{\jmath}=j-\ell_{\varphi}\left\lfloor j / \ell_{\varphi}\right\rfloor$. Clearly, it follows from (50) that if $M_{i, j}=\hat{p}_{\hat{\imath}, j}^{\varphi}$ then $\mathbf{E}\left\{\boldsymbol{\theta}(k+1)=j \mid \mathscr{F}_{k}\right\} \mathbf{1}_{\{\boldsymbol{\theta}(k)=i\}} \leq M_{i, j} \mathbf{1}_{\{\boldsymbol{\theta}(k)=i\}}$ for every $i, j \in \mathcal{I}_{\ell_{O}}$. Furthermore, it follows from the definition of $\hat{\imath}$ and $\hat{\jmath}$ that $M_{i, j}=M_{i+\ell_{\varphi}, j}, M_{i, j+\ell_{\varphi}}=M_{i+\ell_{\varphi}, j+\ell_{\varphi}}, i, j=0, \ldots, \ell_{\varphi}-1$. So $M_{\theta}=\left[M_{i, j}\right]$ can be expressed as $M_{\theta}=E_{\ell_{\nu}} \otimes \Pi_{\varphi}$. Since by construction $M_{\theta}$ is an upper bound matrix, 
then it follows from Theorem III.5.2 that if $\rho\left(\mathcal{A}_{M_{\theta}}\right)<1$ then the jump linear closedloop system embedded in (44) is second moment stable. However, the mean stability characteristics of the jump linear closed-loop system and the HJLS are equivalent. Thus, if $\rho\left(\mathcal{A}_{M_{\theta}}\right)<1$, the HJLS is second moment stable.

Note that $\mathcal{A}_{M_{\theta}}=\mathcal{A}$ (see (45)), so $\rho\left(\mathcal{A}_{M_{\theta}}\right) \leq\left\|\mathcal{A}_{M_{\theta}}\right\|_{\infty}=\|\mathcal{A}\|_{\infty}$. Thus, Theorem III.5.3 provides, in general, a tighter sufficient mean square stability condition than Theorem III.5.1. Furthermore, it also provides a more complete stability characterization because it shows that system (44) is not only mean square stable but also exponentially mean square stable and stochastically stable.

\section{Case Study 2: Stability of a JLS driven by a Static Function of a Markov Chain}

Consider a jump linear system driven by a process $\{\boldsymbol{\theta}(k), k \geq 0\}$, which is a static function of a Markov chain. This is the case, for example, of a jump linear system driven by the output of either a FSM or a finite-state stochastic automaton, which in turn is driven by a Markov chain. These systems have been used to study the stability and performance of fault-tolerant digital flight controllers. For instance, in (Zhang et al. 2005) the stability of these systems was studied using a well-known stability condition which is both necessary and sufficient. In contrast, Theorem III.5.2 provides only a sufficient stability condition. Nevertheless, as will be shown in the sequel, this latter condition requires a much smaller computational effort than the one in (Zhang et al. 2005), and thus is more feasible for use in complex applications. This conclusion is based on the following lemma. 
Lemma III.5.3 Let $\{\varphi(k), k \geq 0\}$ be a finite-state Markov chain with state space $\mathcal{I}_{\ell_{\varphi}}$, transition probability matrix $\Pi_{\varphi}=\left[p_{i, j}^{\varphi}\right]$, and initial distribution $\mu_{0}^{\varphi}$. Let $\{\boldsymbol{\theta}(k), k \geq 0\}$ be such that $\boldsymbol{\theta}(k)=\varpi(\varphi(k)), k \geq 0$, where $\varpi: \mathcal{I}_{\ell_{\varphi}} \rightarrow \mathcal{I}_{\ell_{O}}$ is a static (memoryless) onto map. Assume that $\ell_{O} \leq \ell_{\varphi}$ and, for every $i \in \mathcal{I}_{\ell_{O}}$, define the set $\mathcal{L}_{i} \triangleq \varpi^{-1}(i)$ with $\mathcal{L}_{i} \subset \Sigma_{S}$. The matrix $M_{\theta} \triangleq\left[M_{i, j}\right]$, where

$$
M_{i, j} \triangleq \max _{r \in \mathcal{L}_{i}}\left\{\sum_{s \in \mathcal{L}_{j}} p_{r, s}^{\varphi}\right\}
$$

with $p_{r, s}^{\varphi}=\operatorname{Pr}\{\varphi(k+1)=s \mid \varphi(k)=r\}$, is an upper bound matrix with respect to the random variables defined in (50). That is, $\mathbf{E}\left\{\mathbf{1}_{\{\boldsymbol{\theta}(k+1)=j\}} \mid \mathscr{F}_{k}\right\} \mathbf{1}_{\{\boldsymbol{\theta}(k)=i\}} \leq M_{i, j} \mathbf{1}_{\{\boldsymbol{\theta}(k)=i\}}$ for every $i, j \in \mathcal{I}_{\ell_{O}}$. Moreover, $M_{i, j}$ are the smallest constants that satisfy this condition.

Proof: Let $\mathscr{F}_{k}=\sigma(\boldsymbol{\theta}(0), \ldots, \boldsymbol{\theta}(k))$ and observe that $\mathbf{1}_{\{\boldsymbol{\theta}(k)=i\}}=\sum_{r \in \mathcal{L}_{i}} \mathbf{1}_{\{\boldsymbol{\varphi}(k)=r\}}$. Hence,

$$
\mathbf{E}\left\{\mathbf{1}_{\{\theta(k+1)=j\}} \mid \mathscr{F}_{k}\right\} \mathbf{1}_{\{\theta(k)=i\}}=\sum_{s \in \mathcal{L}_{j}} \sum_{r \in \mathcal{L}_{i}} \mathbf{E}\left\{\mathbf{1}_{\{\varphi(k+1)=s\}} \mid \mathscr{F}_{k}\right\} \mathbf{1}_{\{\varphi(k)=r\}}
$$

Since $\varphi(k)$ is a Markov chain, it follows that

$$
\mathbf{E}\left\{\mathbf{1}_{\{\varphi(k+1)=s\}} \mid \mathscr{F}_{k}\right\} \mathbf{1}_{\{\varphi(k)=r\}}=p_{r, s}^{\varphi} \mathbf{1}_{\{\varphi(k)=r\}},
$$

and therefore,

$$
\mathbf{E}\left\{\mathbf{1}_{\{\boldsymbol{\theta}(k+1)=j\}} \mid \mathscr{F}_{k}\right\} \mathbf{1}_{\{\theta(k)=i\}}=\sum_{r \in \mathcal{L}_{i}}\left\{\sum_{s \in \mathcal{L}_{j}} \hat{p}_{r, s}\right\} \mathbf{1}_{\{\boldsymbol{\varphi}(k)=r\}} .
$$

Observe from (55) that $\sum_{s \in \mathcal{L}_{j}} p_{r, s}^{\varphi} \leq M_{i, j}$, so clearly

$$
\mathrm{E}\left\{\mathbf{1}_{\{\theta(k+1)=j\}} \mid \mathscr{F}_{k}\right\} \mathbf{1}_{\{\theta(k)=i\}} \leq \sum_{r \in \mathcal{L}_{i}} M_{i, j} \mathbf{1}_{\{\varphi(k)=r\}}=M_{i, j} \mathbf{1}_{\{\theta(k)=i\}}
$$




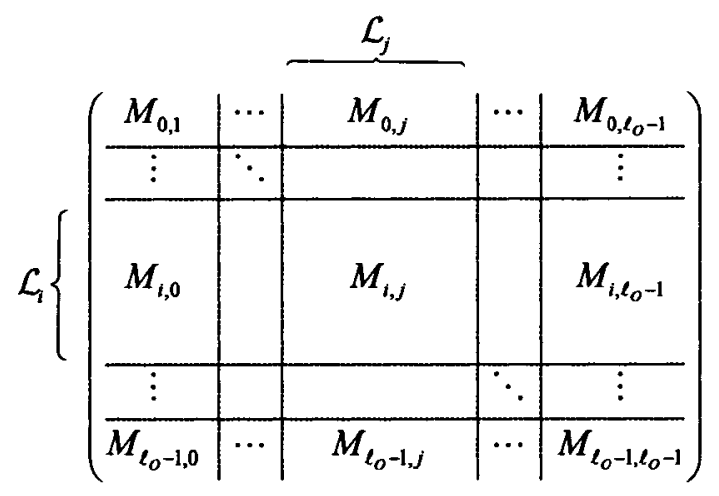

FIG. 10: Structure of $\hat{\Pi}_{\varphi}$, the permuted transition probability matrix of $\varphi(k)$.

Thus, $M_{\theta}=\left[M_{i, j}\right]$ is an upper bound matrix. It also follows from (56) that $M_{i, j}$ is the smallest number that upper bounds all the coefficients $\sum_{s \in \mathcal{L}_{j}} p_{r, s}^{\varphi}$ at every time k. Thus, $M_{i, j}$ is the smallest constant that satisfies $\mathbf{E}\left\{\mathbf{1}_{\{\boldsymbol{\theta}(k+1)=j\}} \mid \mathscr{F}_{k}\right\} \mathbf{1}_{\{\boldsymbol{\theta}(k)=i\}} \leq$ $M_{i, j} \mathbf{1}_{\{\boldsymbol{\theta}(k)=i\}}$. This completes the proof.

Lemma III.5.3 has the following alternative interpretation. Suppose that the rows of $\Pi_{\varphi}$ were reordered so that those corresponding to states in $\mathcal{L}_{0}$ are listed first, those corresponding to states in $\mathcal{L}_{1}$ are listed next, and so on. Moreover, just for convenience, suppose that the rows corresponding to states in the same set, e.g. $\mathcal{L}_{i}$, were reordered in increasing numerical value. Finally, suppose that the columns of $\Pi_{\varphi}$ were then permuted in the same order as the rows and call the permuted matrix $\hat{\Pi}_{\varphi}$. Then $\hat{\Pi}_{\varphi}$ can be partitioned into $\left(\ell_{O}\right)^{2}$ sub-matrices, such that the $i, j$-th sub-matrix, $\hat{\Pi}_{i, j}$, has dimension $n_{i} \times n_{j}$, where $n_{i}=\left|\mathcal{L}_{i}\right|$ (see Figure 10). Then, it follows from (55) and (56) that $M_{i, j}$ is the maximum row sum of $\hat{\Pi}_{i, j}$. Also observe that $M_{i, j}$ represents the maximum probability that $\{\boldsymbol{\theta}(k), k \geq 0\}$ visits state $j$ given that it is 


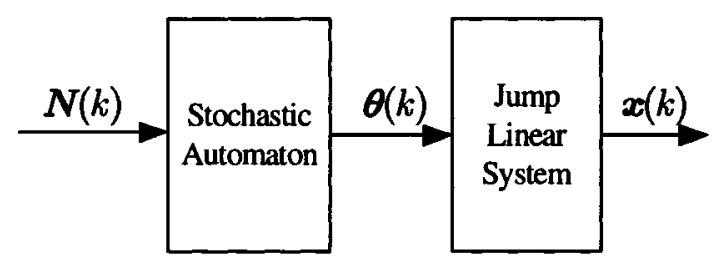

FIG. 11: Jump linear system driven by a stochastic automaton.

currently at state $i$.

In order to relate Lemma III.5.3 with the approach in (Zhang et al. 2005), consider again the jump linear system

$$
\boldsymbol{x}(k+1)=A_{\boldsymbol{\theta}(k)} \boldsymbol{x}(k),
$$

where $\{\boldsymbol{\theta}(k), k \geq 0\}$ is the output of a finite-state stochastic automaton defined as follows (see Figure 11).

Definition III.5.2 A finite-state stochastic automaton is a five-tuple $\left(\mathcal{I}_{\ell_{N}}, \Sigma_{S}, \mathcal{I}_{\ell_{O}}, f, \varpi\right)$, where $\mathcal{I}_{\ell_{N}}$ is the automaton's set of input symbols, $\Sigma_{S}=$ $\left\{e_{1}, \ldots, e_{\ell_{S}}\right\}$ is its set of internal states, and $\mathcal{I}_{\ell_{O}}$ is its set of output symbols. $f$ describes the dynamics of the automaton as follows

$$
\begin{aligned}
f: \mathcal{I}_{\ell_{N}} \times \Sigma_{S} \times \Sigma_{S} & \rightarrow[0,1] \\
\left(l, e_{i}, e_{j}\right) & \mapsto p_{i, j}^{l},
\end{aligned}
$$

where $p_{i, j}^{l} \triangleq \operatorname{Pr}\left\{\boldsymbol{z}(k+1)=e_{j} \mid \boldsymbol{N}(k)=l, \boldsymbol{z}(k)=e_{i}\right\}$, and $\{\boldsymbol{N}(k), k \geq 0\}$ and 
$\{z(k), k \geq 0\}$ represent, respectively, the automaton's input and state processes. Finally, $\varpi$ is a memoryless output map, which has the form

$$
\begin{aligned}
& \varpi: \mathcal{I}_{\ell_{N}} \times \Sigma_{S} \rightarrow \mathcal{I}_{\ell_{O}} \\
& (\boldsymbol{N}(k), \boldsymbol{z}(k)) \mapsto \boldsymbol{\theta}(k)=\varpi(\boldsymbol{N}(k), \boldsymbol{z}(k))=\left[\begin{array}{lll}
0 & \ldots & \left.\ell_{S}-1\right]
\end{array}\right] \boldsymbol{z}(k),
\end{aligned}
$$

that is, the automaton's output process $\{\boldsymbol{\theta}(k), k \geq 0\}$ depends only on its state process, $\{\boldsymbol{z}(k), k \geq 0\}$

It follows from its definition that the automaton is a Moore FSM with nondeterministic state transitions. That is, given a current state and input, eg. $\left(e_{i}, l\right)$, the next state, $e_{j}$, is reached with probability $p_{i, j}^{l}$. Also note that when $\{N(k), k \geq 0\}$ is a Markov chain independent of the automaton's initial state $\boldsymbol{z}(0)$ with transition probability matrix $\Pi_{N}$, the process $\{\boldsymbol{\varphi}(k)=(\boldsymbol{N}(k), \boldsymbol{z}(k)), k \geq 0\}$ is a Markov chain with transition probability matrix given by (Zhang et al. 2005)

$$
\Pi_{\varphi}=\operatorname{diag}\left(\Pi^{0}, \ldots, \Pi^{\ell_{N}-1}\right)\left(\Pi_{N} \otimes I_{\ell_{S}}\right)
$$

where $I_{\ell_{S}}$ is an $\ell_{S} \times \ell_{S}$ identity matrix, and $\Pi^{l}=\left[p_{i, j}^{l}\right] \in \mathbb{R}^{\ell_{S} \times \ell_{S}}$ for all $l \in \mathcal{I}_{\ell_{N}}$. It follows from the definition of $\varpi$ that $\boldsymbol{\theta}(k)=\left[\begin{array}{lllll}0 & 1 & \ldots & \ell_{S}-1\end{array}\right] \varphi(k)$. That is, $\boldsymbol{\theta}(k)$ is a static function of $\varphi(k)$. This motivates the following result.

Lemma III.5.4 Consider the jump linear system (57) driven by the output of a finite-state stochastic automaton as shown in Figure 11. If the input process $\{N(k), k \geq 0\}$ is a Markov chain independent of the automaton's initial condition $\boldsymbol{z}(0)$, then (57) is mean square stable whenever $\rho\left(\mathcal{A}_{M_{\theta}}\right)<1$, where

$$
\mathcal{A}_{M_{\theta}}=\left(M_{\theta}^{\mathrm{T}} \otimes I_{n^{2}}\right) \operatorname{diag}\left(A_{0} \otimes A_{0}, \ldots, A_{\ell_{O}-1} \otimes A_{\ell_{O}-1}\right)
$$

with $M_{\theta}=\left[M_{i, j}\right], M_{i, j}=\max _{l \in \mathcal{I}_{\ell_{N}}}\left\{p_{i+1, j+1}^{l}\right\}, i, j \in \mathcal{I}_{\ell_{O}}$ 
Proof: Since $\{\boldsymbol{\theta}(k), k \geq 0\}$ is a static function of $\{\varpi(k), k \geq 0\}$, it follows from Lemma III.5.3 that $M_{\theta}=\left[M_{i, j}\right]$ is an upper bound matrix provided that

$$
M_{i, j}=\max _{\left(l, e_{r}\right) \in \mathcal{L}_{i}}\left\{\sum_{\left(u, e_{s}\right) \in \mathcal{L}_{j}} \operatorname{Pr}\left\{(\boldsymbol{N}(k+1), \boldsymbol{z}(k+1))=\left(u, e_{s}\right) \mid(\boldsymbol{N}(k), \boldsymbol{z}(k))=\left(l, e_{r}\right)\right\}\right\}
$$

where $\mathcal{L}_{i}=\varpi^{-1}(i)=\left\{\left(l, e_{r}\right) \in \mathcal{I}_{\ell_{N}} \times \Sigma_{S}: \varpi\left(l, e_{r}\right)=i\right\}$, and $\mathcal{L}_{j}=\varpi^{-1}(j)=$ $\left\{\left(u, e_{s}\right) \in \mathcal{I}_{\ell_{N}} \times \Sigma_{S}: \varpi\left(u, e_{s}\right)=j\right\}$. Observe from the structure of the $\varpi$ that $\mathcal{L}_{i}=$ $\left\{\left(0, e_{i+1}\right), \ldots,\left(\ell_{N}-1, e_{i+1}\right)\right\}$ and $\mathcal{L}_{j}=\left\{\left(0, e_{j+1}\right), \ldots,\left(\ell_{N}-1, e_{j+1}\right)\right\}$. Consequently

$$
\begin{aligned}
M_{i, j} & =\max _{l \in \mathcal{I}_{\ell_{N}}}\left\{\sum_{u \in \mathcal{I}_{\ell_{N}}} \operatorname{Pr}\left\{(\boldsymbol{N}(k+1), \boldsymbol{z}(k+1))=\left(u, e_{j+1}\right) \mid(\boldsymbol{N}(k), \boldsymbol{z}(k))=\left(l, e_{i+1}\right)\right\}\right\} \\
& =\max _{l \in \mathcal{I}_{\ell_{N}}}\left\{\operatorname{Pr}\left\{\boldsymbol{z}(k+1)=e_{j+1} \mid(\boldsymbol{N}(k), \boldsymbol{z}(k))=\left(l, e_{i+1}\right)\right\}\right\} \\
& =\max _{l \in \mathcal{I}_{\ell_{N}}}\left\{p_{i+1, j+1}^{l}\right\} .
\end{aligned}
$$

Thus, $M_{\theta}=\left[M_{i, j}\right], M_{i, j}=\max _{l \in \mathcal{I}_{\ell_{N}}}\left\{p_{i+1, j+1}^{l}\right\}, i, j \in \mathcal{I}_{\ell_{O}}$, is equivalent to the upper bound matrix defined in (55). Therefore, it follows from Theorem III.5.2 that (57) is MS stable whenever $\rho\left(\mathcal{A}_{M_{\theta}}\right)<1$.

Note that in (Zhang et al. 2005) the stability of (57) is determined by analyzing the equivalent model

$$
\tilde{\boldsymbol{x}}(k+1)=\tilde{A}_{\boldsymbol{N}(k), \boldsymbol{z}(k)} \tilde{\boldsymbol{x}}(k),
$$

where $\tilde{\boldsymbol{x}}(k) \in \mathbb{R}^{n}$ and $\tilde{A}_{\left(l, e_{i}\right)}=A_{i-1}$, for all $l \in \mathcal{I}_{\ell_{N}}$ and $e_{i} \in \Sigma_{S}$. Since $\{(\boldsymbol{N}(k), \boldsymbol{z}(k)), k \geq 0\}$ is a Markov chain, the mean square stability of (58) can be assessed by determining if the spectral radius of

$$
\mathcal{A}=\left(\Pi_{\varphi}^{\mathrm{T}} \otimes I_{n^{2}}\right) \operatorname{diag}\left(A_{0, e_{1}} \otimes A_{0, e_{1}}, \ldots, A_{\ell_{N}-1, e_{\ell_{S}}} \otimes A_{\ell_{N}-1, e_{\ell_{S}}}\right)
$$


is less than 1. The key observation is that $M_{\theta} \in \mathbb{R}^{\ell_{O} \times \ell_{O}}$, whereas $\Pi_{\varphi} \in \mathbb{R}^{\ell_{N} \ell_{S} \times \ell_{N} \ell_{S}}$. In general $\ell_{N} \ell_{S} \gg \ell_{O}$, so $\rho\left(\mathcal{A}_{M_{\theta}}\right)$ can be computed with much better numerical accuracy than $\rho(\mathcal{A})$. This is a significant advantage in situations where $n \gg 1$. However, as Lemma III.5.4 shows, $M_{\theta}$ does not take the transition probability matrix of $\{\boldsymbol{N}(k), k \geq 0\}$ into consideration, so the stability test in Theorem III.5.2 can be more conservative than the approach taken in (Zhang et al. 2005).

\section{III.6 NUMERICAL EXAMPLES}

This section contains two numerical examples that illustrate the theorems developed in the previous sections. The first example compares Theorems III.3.3, III.3.4, and III.5.1. The second example illustrates Theorem III.5.2.

\section{III.6.1 Example 1: HJLS with Performance Map}

In this example, three hybrid jump linear systems with performance map are used to represent closed-loop systems implemented on fault recoverable computers. These

systems operate as follows: $\{\boldsymbol{N}(k), k \geq 0\}$ is a two-state Markov chain, i.e. $\boldsymbol{N}(k) \in$ $\{0,1\}$, representing the absence $(0)$ or presence (1) of computer faults in a system. The objective of the supervisor is to maintain a correct level of performance, which is attained when the norm of the plant's state vector, $\|x(k)\|$, is below a specified level $\alpha$. Thus, the $A / S$ output is 0 whenever $\|\boldsymbol{x}(k)\|<\alpha$ and 1 otherwise. The supervisor can select one of three operational modes: Nominal, Fault-Recovery, and Performance Recovery. The Nominal mode is selected only when there are no detected faults in the system and the performance is acceptable, i.e., when $\boldsymbol{N}(k)=\boldsymbol{\nu}(k)=0$. The Fault-Recovery mode is selected whenever there is a fault in the system, regardless of 
TABLE I: Hybrid Jump Linear Systems' Parameters.

\begin{tabular}{|c|c|c|c|}
\hline & HJLS 1 & HJLS 2 & HJLS 3 \\
\hline$A_{0}$ & $\begin{array}{ll}0.75 & 0.15 \\
0.40 & 0.30 \\
\end{array}$ & $\begin{array}{rr}0.41 & 0.075 \\
0.20 & 0.15\end{array}$ & $\begin{array}{ll}0.90 & 0.15 \\
0.40 & 0.30\end{array}$ \\
\hline$A_{1}$ & $\begin{array}{rr}-0.62 & 0.00 \\
0.00 & 0.57\end{array}$ & {$\left[\begin{array}{rr}-1.01 & 0.00 \\
0.00 & 0.40\end{array}\right.$} & {$\left[\begin{array}{rr}-1.20 & 0.00 \\
0.00 & 0.70\end{array}\right.$} \\
\hline$A_{2}$ & $\begin{array}{ll}0.21 & 0.00 \\
0.10 & 0.30\end{array}$ & $\begin{array}{ll}0.25 & 0.00 \\
0.05 & 0.30\end{array}$ & $\begin{array}{ll}0.50 & 0.00 \\
0.10 & 0.60\end{array}$ \\
\hline$\Pi_{N}$ & $\begin{array}{ll}0.10 & 0.90 \\
0.05 & 0.95\end{array}$ & $\begin{array}{ll}0.70 & 0.30 \\
0.60 & 0.40\end{array}$ & $\begin{array}{ll}0.60 & 0.40 \\
0.50 & 0.50\end{array}$ \\
\hline
\end{tabular}

the current performance of the system. This is because the supervisor prioritizes fault correction over performance correction. Finally, the Performance Recovery mode is selected when the performance is unacceptable and there is no detected fault present in the system, i.e. $\boldsymbol{N}(k)=0, \boldsymbol{\nu}(k)=1$. The behavior described above can be represented by setting $A_{1}=A_{3}$ in (44). Table I gives the parameters for each HJLS. All the examples use the same initial condition $x_{0}=\left[\begin{array}{ll}1 & -1\end{array}\right]^{T}$, the same initial chain distribution $\mu_{0}^{N}=\left[\begin{array}{ll}1 & 0\end{array}\right]$, and the same performance threshold $\alpha=1$.

In Table II, the entries with a check mark indicate which stability conditions are met by the HJLS's. For example, HJLS 2 satisfies the necessary condition for stability under arbitrary switching but not the sufficient condition clearly showing that the necessary condition is not sufficient to ensure stability under arbitrary switching. Also, HJLS 3 only satisfies the necessary condition for stability under arbitrary switching.

Monte Carlo simulations were used to confirm these predictions. Since it is impossible to show that HJLS 1 is asymptotically stable for every switching rule, the following procedure was followed: 2000 sample paths of $N(k)$ were produced and for 
TABLE II: Stability Conditions Satisfied by the HJLS.

\begin{tabular}{|c|c|c|c|}
\hline & Theorem III.3.3 & Theorem III.3.4 & Theorem III.5.1 \\
\cline { 2 - 4 } & $\rho(\overline{\mathcal{A}})<1$ & $\rho(\overline{\mathcal{A}} / L)<1$ & $\|\mathcal{A}\|_{\infty}<1$ \\
\hline HJLS 1 & $0.9997 \checkmark$ & $0.2499 \checkmark$ & $0.7463 \checkmark$ \\
\hline HJLS 2 & 2.2729 & $0.5682 \checkmark$ & $0.9947 \checkmark$ \\
\hline HJLS 3 & 3.1651 & $0.7913 \checkmark$ & 2.0470 \\
\hline
\end{tabular}

each one, the system trajectory was computed. At every time $k$ the square of the norm of the plant's state vector was calculated. Finally, the average of $\|x(k)\|^{2}$ was computed and plotted in Figure 12. As this figure shows (and was expected), none of the 2000 sample paths diverged to infinity. The mean square stabilities of HJLS's 2 and 3 were estimated also using 2000 Monte Carlo runs. Figure 12 also shows the simulation results for those systems. Note that the simulations suggest that HJLS is also mean square stable.

\section{III.6.2 Example 2: A JLS Driven by a Stochastic Automaton}

Consider a scalar version of the jump linear system in Figure 11. Its dynamics are given by

$$
\boldsymbol{x}(k+1)=a_{\boldsymbol{\theta}(k)} \boldsymbol{x}(k),
$$

where $\{\boldsymbol{\theta}(k), k \geq 0\}$ is the output sequence of a stochastic automaton with parameters: $\mathcal{I}_{\ell_{N}}=\mathcal{I}_{\ell_{O}}=\{0,1\}, \Sigma_{S}=\left\{e_{1}, e_{2}, e_{3}\right\}$

$$
\Pi^{0}=\left[\begin{array}{lll}
0.50 & 0.25 & 0.25 \\
0.25 & 0.25 & 0.50 \\
0.50 & 0.25 & 0.25
\end{array}\right], \quad \Pi^{1}=\left[\begin{array}{ccc}
0.25 & 0.50 & 0.25 \\
0.25 & 0.25 & 0.50 \\
0.50 & 0.25 & 0.25
\end{array}\right],
$$




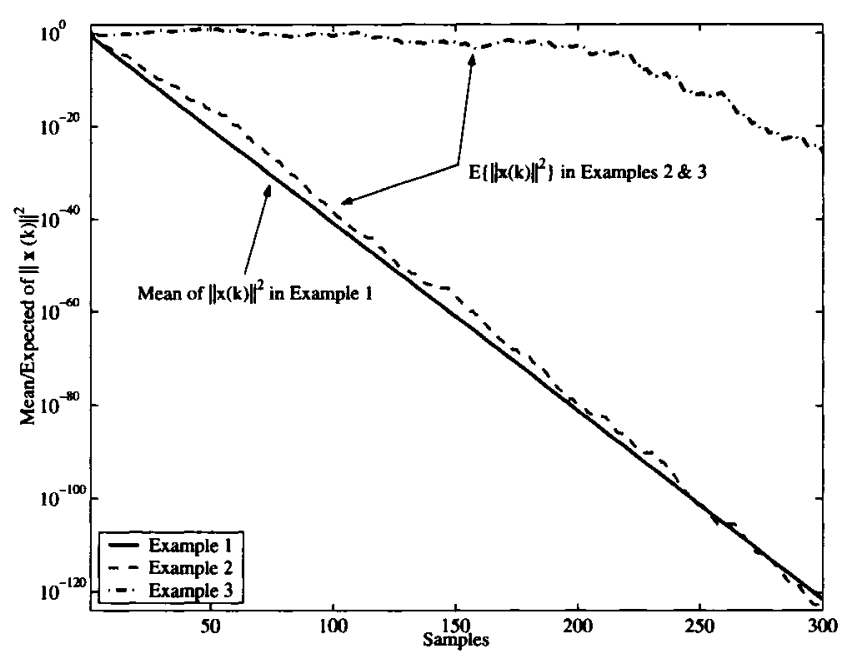

FIG. 12: Mean of the norm squared (HJLS 1) and second moment of $\boldsymbol{x}(k)$ (HJLS's 2 and 3). HJLS 1 satisfies Theorem III.3.3 while HJLS 2 satisfies Theorem III.5.1. HJLS 3 satisfies neither condition.

and

$$
\boldsymbol{\theta}(k)= \begin{cases}0 & : \boldsymbol{z}(k)=e_{1} \\ 1 & : \text { otherwise }\end{cases}
$$

The dynamics of the plant are given by the state matrices $a_{0}=0.7$ and $a_{1}=1.001$. The stability of (60) was determined using Theorem III.5.2 and Lemma III.5.4, and by testing the stability of the equivalent model (58). It follows from Theorem III.5.2 and Lemma III.5.4 that

$$
M_{\theta}=\left[\begin{array}{cc}
0.50 & 0.75 \\
0.5 & 0.75
\end{array}\right], \quad A_{M_{\theta}}=\left[\begin{array}{ll}
0.2450 & 0.5010 \\
0.3675 & 0.7515
\end{array}\right]
$$

Since $\rho\left(\mathcal{A}_{M_{\theta}}\right)=0.9965$, the jump linear system is mean square stable. This was confirmed by Monte Carlo simulations, where the second moment of $\boldsymbol{x}(k)$ was estimated using 10,000 runs each of 500 sample periods. The simulation results are shown in Figure 13, which clearly shows that $\mathbf{E}\left\{\|\boldsymbol{x}(k)\|^{2}\right\} \rightarrow 0$ as $k \rightarrow \infty$ as expected. 


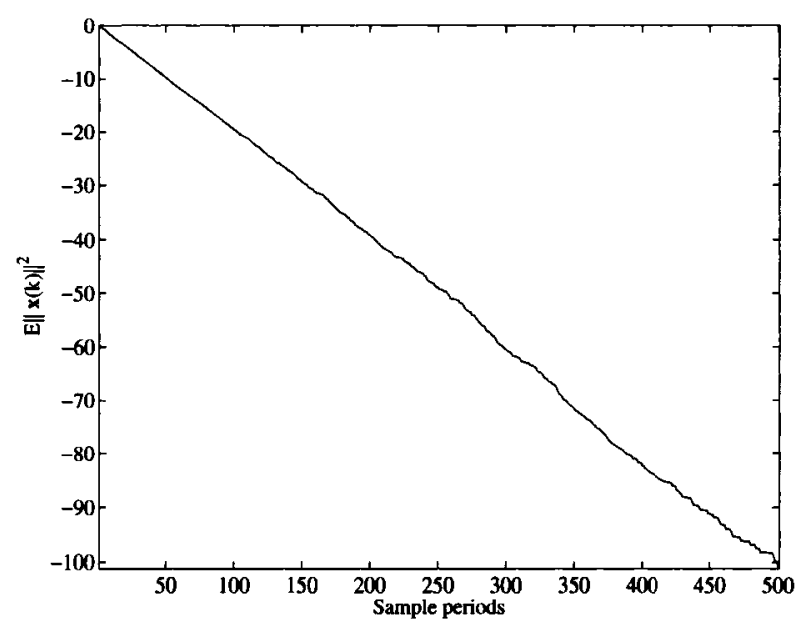

FIG. 13: Estimate of $\mathbf{E}\left\{\|\boldsymbol{x}(k)\|^{2}\right\}$ computed via 10,000 Monte Carlos runs.

The second method, that is, testing the stability of the equivalent model (58), requires one to compute $\rho(\mathcal{A})$, where $\mathcal{A}$ is defined in (59). The spectral radius of $\mathcal{A}$, which varies depending on $\Pi_{N}=\left[\begin{array}{cc}p_{0,0}^{N} & 1-p_{0,0}^{N} \\ 1-p_{1,1}^{N} & p_{1,1}^{N}\end{array}\right]$, was computed for different values of $p_{0,0}^{N}$ and $p_{1,1}^{N}$. The results are plotted in Figure 14. Observe that $0.78 \leq \rho(\mathcal{A}) \leq 0.82$, which implies not only that (60) is mean square stable, but also that $\rho\left(\mathcal{A}_{M_{\theta}}\right)$ and $\rho(\mathcal{A})$ are reasonably close in size.

\section{III.7 CHAPTER SUMMARY AND CONCLUSIONS}

This chapter has established a framework and definitions necessary to study the stability of hybrid jump linear systems. Emphasis was placed in deriving testable sufficient mean square stability conditions, which were derived using three approaches: switched systems techniques, singular value techniques, and a lifting technique. The 


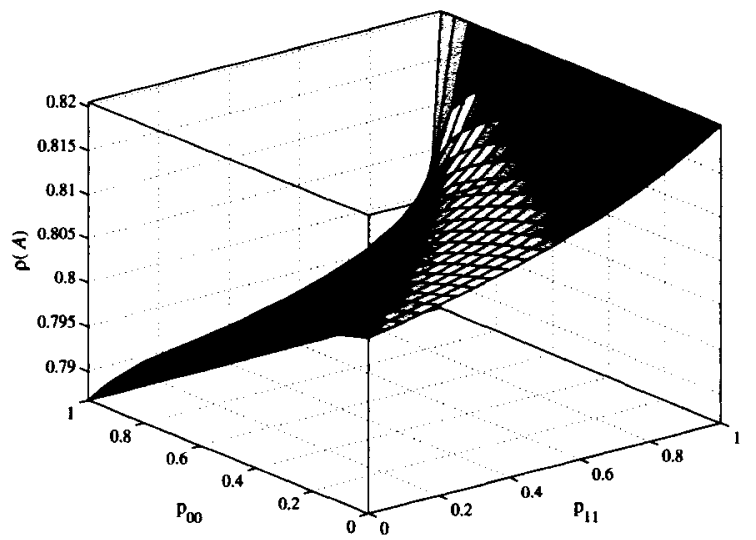

FIG. 14: Spectral radius of $\mathcal{A}$ as a function of $p_{0,0}^{N}$ and $p_{1,1}^{N}$ (see (59)).

main results are listed in Table III along (see also Figure 8). The two more important ideas presented in this chapter follow.

- The stability of a given HJLS can be studied using general techniques, such as those introduced in Theorem III.5.2. However, less conservative conclusions can be drawn by exploiting the particular structure of the HJLS, as exemplified in Theorems III.4.2 and III.4.3.

- Theorem III.5.2 established a general method to study the stability of jump linear system driven by general stochastic processes. 
TABLE III: Summary of The Main Results in Chapter III.

\begin{tabular}{|c|l|l|}
\hline Technique & Theorem & \multicolumn{1}{|c|}{ Summary } \\
\hline \multirow{5}{*}{ Switched Systems } & III.3.3 & Sufficient AAS condition for switched systems \\
\cline { 2 - 4 } & III.3.4 & Necessary AAS condition for switched systems \\
\cline { 2 - 4 } Singular Values & III.3.5 & Sufficient MSS condition for general HJLS's \\
\cline { 2 - 4 } & III.4.2 & $\begin{array}{l}\text { A sufficient and a necessary MSS condition for a } \\
\text { particular HJLS }\end{array}$ \\
\cline { 2 - 4 } & III.4.3 & $\begin{array}{l}\text { Sufficient MS instability condition for a particular } \\
\text { HJLS }\end{array}$ \\
\hline \multirow{2}{*}{ Lifted $\mathcal{A}$ matrix } & III.5.2 & $\begin{array}{l}\text { Sufficient MSS condition for JLS's driven by } \\
\text { general, finite-state, discrete-time stochastic pro- } \\
\text { cesses }\end{array}$ \\
\cline { 2 - 4 } & III.5.1 & $\begin{array}{l}\text { Sufficient MSS condition for a HJLS's with per- } \\
\text { formance map }\end{array}$ \\
\hline
\end{tabular}




\section{CHAPTER IV}

\section{STABILITY ANALYSIS OF HJLS'S USING THE MARKOV KERNEL TECHNIQUE}

\section{IV.1 INTRODUCTION}

The main goal of this chapter is to derive testable mean square stability conditions for HJLS's with embedded Moore FSM's using Theorem III.5.2. As explained in Chapter III, this theorem makes use of the upper bound matrix, $M_{\theta}=\left[M_{i, j}\right]$, which collects the smallest possible upper bounds, $M_{i, j}$, for a particular set of random variables associated with the mode-setting process $\{\boldsymbol{\theta}(k), k \geq 0\}$. When this process is a Markov chain, its transition probability matrix, $\Pi_{\theta}$, and the matrix $M_{\theta}$ coincide. In the context of HJLS's, however, $\{\boldsymbol{\theta}(k), k \geq 0\}$ is generally not a Markov chain, and $M_{\theta}$ must be computed using a different type of analysis. As is shown in the sequel, under specific conditions the state process of a HJLS, $\{(\boldsymbol{y}(k), \boldsymbol{N}(k)), k \geq 0\}$, constitutes a Markov chain on a metric space with associated Markov kernel $\mathrm{P}_{(y, N)}$. This kernel, which determines the transition probabilities of the joint process $\{(\boldsymbol{y}(k), \boldsymbol{N}(k)), k \geq 0\}$, can be used to estimate the transition probabilities of $\{\boldsymbol{\theta}(k), k \geq 0\}$ yielding the smallest possible values for $M_{i, j}$. The end result of this analysis is Theorem IV.4.4, which extends the stability test for HJLS's with performance map, introduced in Chapter III, to a much larger subclass of HJLS's, namely, HJLS's with Moore-type FSM's. 
The concepts and analysis tools needed to prove Theorem IV.4.4 are introduced in Sections IV.2 and IV.3. Of special interest is Theorem IV.2.1, which shows the conditions under which the joint process, $\{(\boldsymbol{x}(k), \boldsymbol{N}(k)), k \geq 0\}$, formed by combining the solution, $\boldsymbol{x}(k)$, of a non-linear stochastic difference equation of the form $\boldsymbol{x}(k+1)=$ $F_{k}(\boldsymbol{x}(k), \boldsymbol{N}(k))$ and its input, $\boldsymbol{N}(k)$, constitutes a Markov chain. This well-known fact plays an important role in the analysis that leads to Theorem IV.4.4. Surprisingly, a detailed proof of Theorem IV.2.1 seems to be lacking in the literature. One is provided here based on the general approach presented (Morozan 1976) for non-linear difference equations with i.i.d. inputs.

As was discussed in Chapter II, HJLS's are autonomous discrete stochastic hybrid automata which, in turn, are known to be the discrete-time version of piecewise deterministic Markov processes (PDP's) (see Bemporad \& Di Cairano 2005). The latter have been extensively studied due to their ability to model a broad range of continuous-time phenomena (cf. Davis 1993). So far, the research on the stability of PDP's (in continuous-time) has mainly addressed conditions for the existence of non-trivial invariant distributions (cf. Davis 1993). Although there is also some work on moment stability for these systems, there are at present no mean square stability tests for continuous-time or discrete-time PDP's. Thus, the ideas presented in the following sections may provide an alternative methodology for the stability analysis of PDP's in discrete-time. The foundations are laid for such a methodology by deriving several properties of HJLS's, including a complete account of the many Markov kernels associated with them, and the evolution of their probability measures.

The rest of the chapter is organized as follows: basic facts about Markov chains 
in metric spaces are summarized at the end of this section. Section IV.2 derives the Markov property for non-linear difference equations with Markovian inputs, Theorem IV.2.1. It also derives the Markov kernel associated with these equations. Section IV.3 derives the properties of HJLS's from the Markov chain perspective. It is shown that a HJLS can be posed in the framework of Theorem IV.2.1, proving that the state process of a HJLS, $\{\boldsymbol{y}(k), k \geq 0\}$, has the Markov property. This section also derives the Markov kernels and the probability distributions associated with HJLS's. The chapter ends with Section IV.4, which introduces two new sets of testable sufficient mean square stability conditions for HJLS's. The first set of conditions consider a special case of the $A / S$ map. Under this restriction, the FSM embedded in a HJLS behaves as if it were driven only by its input $\{N(k), k \geq 0\}$. As a consequence, the mean square stability of the HJLS can be studied with tools available for Markov jump linear systems. The second set of conditions is based on computing the bounds, $M_{i, j}$, in Theorem III.5.2 using the Markov kernels associated with the HJLS. These results constitute the main contributions of this dissertation.

\section{IV.1.1 Basic Properties of Markov Chains in Metric Spaces}

Let $\{\varphi(k), k \geq 0\}$ denote a discrete-time stochastic process taking values on a set $X$. This process is called a Markov chain if it satisfies the Markov property, namely, $\operatorname{Pr}\{\varphi(k+1) \in B \mid \varphi(k), \ldots, \varphi(0)\}=\operatorname{Pr}\{\varphi(k+1) \in B \mid \varphi(k)\}$. Markov chains are usually studied under the assumption that their state spaces are finite. In such cases, their properties can be characterized by studying their one-step transition probabilities (cf. Papoulis 1991). If, for example, $\varphi(k) \in X=\mathcal{I}_{\ell_{\varphi}}$, it is always possible to arrange its transition probabilities into a transition probability matrix, $\Pi_{\varphi}=\left[p_{i, j}^{\varphi}\right]$, 
with $p_{i, j}^{\varphi}=\operatorname{Pr}\{\varphi(k+1)=j \mid \varphi(k)=i\}, i, j \in \mathcal{I}_{\ell_{\varphi}}$, whose properties determine, among other things, the long term behavior of $\{\boldsymbol{\varphi}(k), k \geq 0\}$. This analysis, however, is not practical or even possible when $X=\mathbb{Z}$ or $X=\mathbb{R}$ for obvious cardinality problems. Fortunately, most of the theory available for finite-state Markov chains has been extended, under certain conditions, to Markov chains with countable (cf. Nummelin 1984) and uncountable (cf. Hernández-Lerma \& Lasserre 2003, Meyn \& Tweedie 1993) state spaces. In these cases, the transition probability matrices are replaced by Markov kernels which determine the long term behavior of their associated Markov chains. Since the state process of a HJLS, $\{\boldsymbol{y}(k), k \geq 0$, takes values on $\mathbb{R}^{n+\ell_{S}}$, the rest of this section will summarize the basic properties of countable (or uncountable) Markov chains evolving over metric spaces.

Let $\left(X, d_{X}\right)$ be a metric space with Borel algebra $\mathscr{B}(X)$. A Markov kernel on $X$ is a function, $\mathrm{P}: X \times \mathscr{B}(X) \rightarrow[0,1]$, with the following properties (Hernández-Lerma \& Lasserre 2003):

(MK-i) For every $\chi \in X, \mathrm{P}(\chi, \cdot)$ is a probability measure over $\mathscr{B}(X)$.

(MK-ii) For every $B \in \mathscr{B}(X), \mathrm{P}(\cdot, B)$ is a bounded measurable function.

If $\{\varphi(k), k \geq 0\}$ is a time-homogeneous Markov chain on $X$, it can be shown that the function

$$
\mathrm{P}_{\varphi}(\chi, B) \triangleq \operatorname{Pr}\{\varphi(k+1) \in B \mid \varphi(k)=\chi\}
$$

with $\chi \in X$ and $B \in \mathscr{B}(X)$, satisfies properties MK-i and MK-ii above. This function, called the Markov kernel associated with $\{\varphi(k), k \geq 0\}$, is the countable (or uncountable) equivalent of a transition probability matrix. Moreover, the long 
term behavior of $\{\varphi(k), k \geq 0\}$, that is, its convergence, is determined by its Markov kernel since the chain's probability measures,

$$
\mu_{k}^{\varphi}(B) \triangleq \operatorname{Pr}\{\varphi(k) \in B\}, \quad B \in \mathscr{B}(X), k \geq 0
$$

evolve according to the Kolmogorov equation

$$
\begin{aligned}
\mu_{k+1}^{\varphi}(B) & =\int_{X} \mathrm{P}_{\varphi}\left(\chi_{k}, B\right) \mu_{k}^{\varphi}\left(d \chi_{k}\right) \\
& =\int_{X} \mathrm{P}_{\varphi}\left(\chi_{k}, B\right) \int_{X} \mathrm{P}_{\varphi}\left(\chi_{k-1}, B\right) \mu_{k-1}^{\varphi}\left(d \chi_{k-1}\right) \\
& =\int_{X} \mathrm{P}_{\varphi}\left(\chi_{k}, B\right) \int_{X} P_{\varphi}\left(\chi_{k-1}, B\right) \cdots \int_{X} \mathrm{P}_{\varphi}\left(\chi_{0}, B\right) \mu_{0}^{\varphi}\left(d \chi_{0}\right) .
\end{aligned}
$$

To simplify the notation, let $M_{1}(X)$ represent the set of all the probability measures over $\mathscr{B}(X)$. Next, define the operators $\mathrm{P}_{\varphi}^{k}: M_{1}(X) \rightarrow M_{1}(X), k \geq 0$ as follows:

$$
\mathrm{P}_{\varphi}^{0} \mu(B)=\mu(B), \quad \mathrm{P}_{\varphi} \mu(B)=\int_{X} \mathrm{P}_{\varphi}(y, B) \mu(d y), \quad \mathrm{P}_{\varphi}^{k+1} \mu(B)=\mathrm{P}_{\varphi} \mathrm{P}_{\varphi}^{k} \mu(B),
$$

where $\mu \in M_{1}(X)$ and $B \in \mathscr{B}(X)$. The Kolmogorov equation (62) can now be expressed more succinctly as

$$
\mu_{k+1}^{\varphi}(B)=\mathrm{P}_{\varphi} \mu_{k}^{\varphi}(B)=\mathrm{P}_{\varphi}^{k+1} \mu_{0}^{\varphi}(B)
$$

The importance of equation (64) in stability analysis is twofold. First, if there exist invariant distributions $\mu_{*}^{\varphi}$ such that $\mu_{*}^{\varphi}(B)=\mathrm{P}_{\varphi} \mu_{*}^{\varphi}(B)$, for all $B \in \mathscr{B}(B)$, it can be shown that under certain conditions the long term behavior of $\{\varphi(k), k \geq 0\}$ can be studied via Monte Carlo simulations (Hernández-Lerma \& Lasserre 2003). Second, if it can be shown that there exists a unique invariant distribution, $\mu_{*}^{\varphi}$, with the property

$$
\lim _{k \rightarrow \infty} \int_{X} h(\chi) P_{\varphi}^{k} \mu(d \chi)=\int_{X} h(\chi) \mu_{*}^{\varphi}(d \chi)
$$


for every $\mu \in M_{1}(X)$ and every bounded measurable function $h: X \rightarrow \mathbb{R}$ with compact support, then $\{\varphi(k), k \geq 0\}$ converges at least in distribution, which is the weakest form of stochastic convergence. In other words, the existence of such a unique invariant distribution is a necessary condition for the convergence of $\{\varphi(k), k \geq 0\}$ in, for example, the second moment.

This approach, which is equivalent to the one used to study PDP in continuoustime (Davis 1993), will not be pursued in this chapter. Instead, (64) will be used to derive closed-form formulas for the probability distributions associated with HJLS's (e.g. $\mu_{k}^{y}$ ). The analysis and interpretation of these formulas will lead to the sufficient mean square stability tests introduced in Section IV.4. Towards this goal, the next section introduces the tools necessary to establish the Markov property of the process $\{(\boldsymbol{y}(k), \boldsymbol{N}(k)), k \geq 0\}$, where $\boldsymbol{y}(k)$ is the state of a HJLS and $\{\boldsymbol{N}(k), k \geq 0\}$ is a Markov chain.

\section{IV.2 THE MARKOV PROPERTY OF NON-LINEAR DIFFERENCE EQUATIONS WITH MARKOVIAN INPUTS}

It will be shown in the next section that the dynamics of a hybrid jump linear system can be expressed as the solution of a non-linear difference equation with a Markovian input. Although the Markov property of this type of equations has already been established by several authors, a comprehensive and complete proof of this fact is lacking in the literature. Consequently, the first subsection addresses this problem by introducing a detailed proof of this fact using only fundamental measure-theoretical concepts. The second subsection below presents the Markov kernels associated with 
this type of equations.

\section{IV.2.1 The Markov Property of Difference Equations with Markovian Inputs}

Consider the following system of difference equations

$$
\boldsymbol{x}(k+1)=F_{k}(\boldsymbol{x}(k), \boldsymbol{N}(k))
$$

where $F_{k}$ is a time-indexed sequence of Borel functions and $\{\boldsymbol{N}(k), k \geq 0\}$ is a Markov chain. The Markov property of $\{(\boldsymbol{x}(k), \boldsymbol{N}(k)), k \geq 0\}$ (or $\{(\boldsymbol{x}(t), \boldsymbol{N}(t)), t \geq$ $0\}$ ) for this class of equations has been addressed in the literature. It has been stated without proof by many authors both in discrete-time (cf. Costa et al. 2005) and in continuous-time (cf. Fang \& Loparo 2002, Ehrhardt \& Kleinman 1982). On the other hand, the proofs that are available either address the simpler case when $\{\boldsymbol{N}(k), k \geq 0\}$ (or $\{\boldsymbol{N}(t), t \geq 0\}$ ) is an independent and identically distributed (i.i.d.) process (Morozan 1976, Arnold 1974), use heuristic arguments (Srichander \& Walker 1993), or employ sophisticated results (Arnold \& Kleinmann 1982). To the best of our knowledge, there is no complete proof of the Markov property of $(x(k), N(k))$ when $\boldsymbol{N}(k)$ is a Markov chain. The following theorem presents a complete and explicit proof which uses only fundamental measure-theoretical concepts and follows the probabilistic approach, that is, it interprets the process $\{(x(k), N(k)), k \geq 0\}$ as a sequence of random vectors and exploits its properties.

Theorem IV.2.1 Consider the system of equations

$$
\boldsymbol{x}(k+1)=F_{k}(\boldsymbol{x}(k), \boldsymbol{N}(k)), \quad \boldsymbol{x}(0)=\boldsymbol{x}_{0},
$$


where $\boldsymbol{x}(k) \in \mathbb{R}^{n},\{\boldsymbol{N}(k), k \geq 0\}$ is a Markov chain in $\mathbb{R}^{m}$ with a finite, countable or uncountable state space, $\boldsymbol{x}_{0}$ is an integrable random vector independent of $\{\boldsymbol{N}(k), k \geq$ $0\}$, and $F_{k}: \mathbb{R}^{n} \times \mathbb{R}^{m} \rightarrow \mathbb{R}^{n}$ are measurable functions for every $k \geq 0$. The process $\{(\boldsymbol{x}(k), \boldsymbol{N}(k)), k \geq 0\}$ is a Markov chain.

The proof of Theorem IV.2.1 relies on the following three supporting results: Theorems IV.2.2-IV.2.4. The detailed proofs of Theorems IV.2.2 and IV.2.4 are relegated to Appendix B to simplify the presentation of the proof of Theorem IV.2.1. The proof of Theorem IV.2.4 can be found in the appendix of (Morozan 1976).

Theorem IV.2.2 Consider system (65) under the conditions of Theorem IV.2.1, and let $f: \mathbb{R}^{m} \rightarrow \mathbb{R}$ be any bounded Borel function. Then, it follows that

$$
\mathbf{E}\{f(\boldsymbol{N}(k+1)) \mid \boldsymbol{x}(k), \boldsymbol{N}(k), \ldots, \boldsymbol{x}(0), \boldsymbol{N}(0)\}=\mathbf{E}\{f(\boldsymbol{N}(k+1)) \mid \boldsymbol{x}(k), \boldsymbol{N}(k)\}
$$

Proof Outline: This result is straightforward. Since $\boldsymbol{x}(k)$ is a (measurable) function of $\boldsymbol{\theta}(k-1), \ldots, \boldsymbol{\theta}(0)$, then $\sigma(\boldsymbol{x}(k), \ldots, \boldsymbol{x}(1)) \subset \sigma(\boldsymbol{\theta}(k), \ldots, \boldsymbol{\theta}(0))$. Hence, $\sigma(\boldsymbol{x}(k), \boldsymbol{N}(k), \ldots, \boldsymbol{x}(0), \boldsymbol{N}(0))=\sigma(\boldsymbol{\theta}(k), \ldots, \boldsymbol{\theta}(0), \boldsymbol{x}(0))$. This fact together with the independence of $\boldsymbol{x}(0)$ and $\{\boldsymbol{N}(k), k \geq 0\}$, and the Markov property of $\{\boldsymbol{N}(k), k \geq 0\}$ show (66).

The formal proof of Theorem IV.2.2, which uses measure-theoretic concepts and theorems that need to be introduced first, has been included in Appendix B.

Theorem IV.2.3 (Morozan 1976) Let $\mathcal{H}$ be a linear space of bounded real-valued functions defined on a set $\Phi$. Assume that

(i) $\mathcal{H}$ contains the constant functions. 
(ii) If $\left\{f_{n}\right\}$ is a sequence in $\mathcal{H}$ and $f_{n} \rightarrow f$ uniformly, then $f \in \mathcal{H}$.

(iii) If $\left\{f_{n}\right\}$ is a monotone sequence in $\mathcal{H}$ and $0 \leq f_{n} \leq M$ for all $n$, then $\lim _{n \rightarrow \infty} f_{n} \in \mathcal{H}$

(iv) $\mathcal{H}$ has a subset $\mathcal{C}$ with the property: if $c_{1}, c_{2} \in \mathcal{C}$, then their product, $\mathrm{c}_{1} \mathrm{c}_{2}$, also belongs to $\mathcal{C}$.

Then $\mathcal{H}$ contains every bounded function $g: \Phi \rightarrow \mathbb{R}$ which is measurable with respect to the $\sigma$-algebra generated by the sets: $\{\phi \in \Phi: c(\phi) \in B\}, c \in \mathcal{C}, B \in \mathscr{B}(\mathbb{R})$.

Proof: See Theorem 1 in the appendix of (Morozan 1976).

Theorem IV.2.4 Consider system (65) under the conditions of Theorem IV.2.1, and let $\Phi=\mathbb{R}^{n} \times \mathbb{R}^{m} \times \mathbb{R}^{m}$. If $\mathcal{H}$ is the set of all bounded Borel functions $f: \Phi \rightarrow \mathbb{R}$ such that

$$
\begin{gathered}
\mathbf{E}\{f(\boldsymbol{x}(k), \boldsymbol{N}(k), \boldsymbol{N}(k+1)) \mid \boldsymbol{x}(k), \boldsymbol{N}(k), \ldots, \boldsymbol{x}(0), \boldsymbol{N}(0)\} \\
=\mathbf{E}\{f(\boldsymbol{x}(k), \boldsymbol{N}(k), \boldsymbol{N}(k+1)) \mid \boldsymbol{x}(k), \boldsymbol{N}(k)\}
\end{gathered}
$$

then $\mathcal{H}$ satisfies conditions (i)-(iv) of Theorem IV.2.3.

Proof: See Appendix B for details.

The fundamental step in the proof of Theorem IV.2.1 is to establish that the set $\mathcal{H}$ of all bounded Borel functions which satisfy (67) contains every bounded Borel function on $\Phi$. This is proven by combining Theorems IV.2.3 and IV.2.4. Theorem IV.2.2 is used to verify property (iv) in Theorem IV.2.4.

Proof of Theorem IV.2.1: First recall from (p. 564, Shiryaev 1995) that a random process $\{\varphi(k), k \geq 0\}$ is a Markov chain if and only if for every bounded, realvalued Borel function, $h$, it follows that $\mathbf{E}\{h(\varphi(k+1)) \mid \varphi(k), \ldots, \varphi(0)\}=\mathbf{E}\{h(\varphi(k+$ 
1)) $\mid \boldsymbol{\varphi}(k)\}$. In particular, to show that $(\boldsymbol{x}(k), \boldsymbol{N}(k))$ is a Markov chain, it is sufficient to prove that for every bounded Borel function $h: \mathbb{R}^{n} \times \mathbb{R}^{m} \rightarrow \mathbb{R}$

$$
\begin{aligned}
\mathbf{E}\{h(\boldsymbol{x}(k+1), \boldsymbol{N}(k+1)) \mid \boldsymbol{x}(k), \boldsymbol{N}(k), \ldots, \boldsymbol{x}(0), \boldsymbol{N}(0)\} & \\
& =\mathbf{E}\{h(\boldsymbol{x}(k+1), \boldsymbol{N}(k+1)) \mid \boldsymbol{x}(k), \boldsymbol{N}(k)\}
\end{aligned}
$$

or, equivalently,

$$
\begin{aligned}
\mathbf{E}\left\{h\left(F_{k}(\boldsymbol{x}(k), \boldsymbol{N}(k)), \boldsymbol{N}(k+1)\right) \mid \boldsymbol{x}(k), \boldsymbol{N}(k), \ldots, \boldsymbol{x}(0), \boldsymbol{N}(0)\right\} \\
=\mathbf{E}\left\{h\left(F_{k}(\boldsymbol{x}(k), \boldsymbol{N}(k)), \boldsymbol{N}(k+1)\right) \mid \boldsymbol{x}(k), \boldsymbol{N}(k)\right\} .
\end{aligned}
$$

Let $\mathcal{H}$ be the set of all the bounded Borel functions $f: \Phi \rightarrow \mathbb{R}, \Phi=\mathbb{R}^{n} \times \mathbb{R}^{m} \times \mathbb{R}^{m}$, that satisfies the expression

$$
\begin{aligned}
\mathbf{E}\{f(\boldsymbol{x}(k), \boldsymbol{N}(k), \boldsymbol{N}(k+1)) \mid \boldsymbol{x}(k), & \boldsymbol{N}(k), \ldots, \boldsymbol{x}(0), \boldsymbol{N}(0)\} \\
& =\mathbf{E}\{f(\boldsymbol{x}(k), \boldsymbol{N}(k), \boldsymbol{N}(k+1)) \mid \boldsymbol{x}(k), \boldsymbol{N}(k)\} .
\end{aligned}
$$

It follows from Theorem IV.2.4 that $\mathcal{H}$ satisfies all the conditions of Theorem IV.2.3. Thus, $\mathcal{H}$ contains all the bounded functions $g: \Phi \rightarrow \mathbb{R}$ which are measurable with respect to $\mathscr{A}$, the $\sigma$-algebra generated by the sets $\{\phi \in \Phi: c(\phi) \in B\}, c \in \mathcal{C} \subseteq \mathcal{H}$, $B \in \mathscr{B}(\mathbb{R})$

As is shown in the proof of Theorem IV.2.4, the set $\mathcal{C}$ is composed of all bounded, separable functions $c: \Phi \rightarrow \mathbb{R}$ of the form $c(\phi)=\hat{c}_{1}(\gamma) \hat{c}_{2}(\lambda)$, where $\hat{c}_{1}: \mathbb{R}^{n} \times \mathbb{R}^{m} \rightarrow \mathbb{R}$ and $\hat{c}_{2}: \mathbb{R}^{m} \rightarrow \mathbb{R}$ are bounded Borel functions, and $\phi=(\gamma, \lambda)$.

Now, let $A \in \mathscr{A}$ and observe that $A$ must be of the form

$$
A=\bigcup_{i, j}\left\{\phi: c_{i}(\phi) \in B_{i_{j}}\right\}
$$


for some $c_{i} \in \mathcal{C}$ and $B_{i_{j}} \in \mathscr{B}(\mathbb{R})$. But the functions $c_{i}$ are Borel, thus $\left\{\phi: c_{i}(\phi) \in\right.$ $\left.B_{i_{j}}\right\} \in \mathscr{B}\left(\mathbb{R}^{n}\right) \otimes \mathscr{B}\left(\mathbb{R}^{m}\right) \otimes \mathscr{B}\left(\mathbb{R}^{m}\right)$, and consequently, $A \in \mathscr{B}\left(\mathbb{R}^{n}\right) \otimes \mathscr{B}\left(\mathbb{R}^{m}\right) \otimes \mathscr{B}\left(\mathbb{R}^{m}\right) .^{1}$ Moreover, since this is true for every $A \in \mathscr{A}$, it follows that $\mathscr{A} \subseteq \mathscr{B}\left(\mathbb{R}^{n}\right) \otimes \mathscr{B}\left(\mathbb{R}^{m}\right) \otimes$ $\mathscr{B}\left(\mathbb{R}^{m}\right)$. Conversely, note that for every $B \in \mathscr{B}\left(\mathbb{R}^{n}\right) \otimes \mathscr{B}\left(\mathbb{R}^{m}\right) \otimes \mathscr{B}\left(\mathbb{R}^{m}\right), B=\{\phi$ : $\left.\mathbf{1}_{\{B\}}(\phi) \in[0.5,1.5)\right\} \in \mathscr{A}$, since the indicator function of $B, \mathbf{1}_{\{B\}}(\cdot)$, belongs to $\mathcal{C}$. This implies that $\mathscr{A} \supseteq \mathscr{B}\left(\mathbb{R}^{n}\right) \otimes \mathscr{B}\left(\mathbb{R}^{m}\right) \otimes \mathscr{B}\left(\mathbb{R}^{m}\right)$, so $\mathscr{A}=\mathscr{B}\left(\mathbb{R}^{n}\right) \otimes \mathscr{B}\left(\mathbb{R}^{m}\right) \otimes \mathscr{B}\left(\mathbb{R}^{m}\right)$.

Hence, $\mathcal{H}$ contains every bounded Borel function of the form $g: \Phi \rightarrow \mathbb{R}$, and each of these functions satisfies (68). In particular, let $h: \mathbb{R}^{n} \times \mathbb{R}^{m} \rightarrow \mathbb{R}$ be any bounded Borel function and define $g(\phi)=h\left(F_{k}(\gamma), \lambda\right), \phi=(\gamma, \lambda)$. Clearly, $g \in \mathcal{H}$, so in (68)

$$
\begin{aligned}
\mathrm{E}\{g(\boldsymbol{x}(k), \boldsymbol{N}(k), \boldsymbol{N}(k+1)) \mid \boldsymbol{x}(k), \boldsymbol{N}(k), \ldots, \boldsymbol{x}(0), \boldsymbol{N}(0)\} & \\
& =\mathrm{E}\{g(\boldsymbol{x}(k), \boldsymbol{N}(k), \boldsymbol{N}(k+1)) \mid \boldsymbol{x}(k), \boldsymbol{N}(k)\},
\end{aligned}
$$

which implies that

$$
\begin{aligned}
\mathrm{E}\left\{h\left(F_{k}(\boldsymbol{x}(k), \boldsymbol{N}(k)), \boldsymbol{N}(k+1)\right) \mid \boldsymbol{x}(k), \boldsymbol{N}(k), \ldots, \boldsymbol{x}(0), \boldsymbol{N}(0)\right\} \\
=\mathrm{E}\left\{h\left(F_{k}(\boldsymbol{x}(k), \boldsymbol{N}(k)), \boldsymbol{N}(k+1)\right) \mid \boldsymbol{x}(k), \boldsymbol{N}(k)\right\},
\end{aligned}
$$

or

$$
\begin{aligned}
\mathbf{E}\{h(\boldsymbol{x}(k+1), \boldsymbol{N}(k+1)) \mid \boldsymbol{x}(k), \boldsymbol{N}(k), \ldots, \boldsymbol{x}(0), \boldsymbol{N}(0)\} & \\
& =\mathbf{E}\{h(\boldsymbol{x}(k+1), \boldsymbol{N}(k+1)) \mid \boldsymbol{x}(k), \boldsymbol{N}(k)\} .
\end{aligned}
$$

Since this is true for every bounded Borel function $h: \mathbb{R}^{n} \times \mathbb{R}^{m} \rightarrow \mathbb{R}$, we conclude that $\{(\boldsymbol{x}(k), \boldsymbol{N}(k)), k \geq 0\}$ is a Markov chain.

The next step in the analysis is to derive the Markov kernel associated with $\{(\boldsymbol{x}(k), \boldsymbol{N}(k)), k \geq 0\}$.

\footnotetext{
${ }^{1}$ In this context, $\otimes$ represents the direct product of $\sigma$-algebras.
} 


\section{IV.2.2 The Markov Kernel of Non-Linear Difference Equations with Markovian Inputs}

The following two assumptions are introduced to place the following discussion closer to the context of HJLS. First, although the proof of Theorem IV.2.1 does not require $\{\boldsymbol{N}(k), k \geq 0\}$ to be a finite-state Markov chain, it will be assumed that it takes values from $\mathcal{I}_{\ell_{N}}$ with transition probability matrix $\Pi_{N}=\left[p_{i, j}^{N}\right]$. Second, it will be assumed that $F_{k}=F$ for all $k \geq 0$. Under these conditions, the Markov kernel associated with $\{(\boldsymbol{x}(k), \boldsymbol{N}(k)), k \geq 0\}, \mathrm{P}_{(x, N)}$, can be derived using the approach introduced in subsection IV.1.1. That is, define

$$
\mathrm{P}_{(x, N)}((x, i), D) \triangleq \operatorname{Pr}\{(\boldsymbol{x}(k+1), \boldsymbol{N}(k+1)) \in D \mid(\boldsymbol{x}(k), \boldsymbol{N}(k))=(x, i)\}
$$

for every $(x, i) \in \mathbb{R}^{n} \times \mathcal{I}_{\ell_{N}}$ and $D \in \mathscr{B}\left(\mathbb{R}^{n} \times \mathcal{I}_{\ell_{N}}\right)$. Observe that $\operatorname{Pr}\{(\boldsymbol{x}(k+1), \boldsymbol{N}(k+$ 1)) $\in D \mid(\boldsymbol{x}(k), \boldsymbol{N}(k))=(x, i)\} \neq 0$ only if $(F(x, i), l) \in D$ for some $l \in \mathcal{I}_{\ell_{N}}$. Note that $\{\omega: \boldsymbol{x}(k, \omega)=x, \boldsymbol{N}(k, \omega)=i\}=\{\omega: \boldsymbol{x}(k+1, \omega)=F(x, i)\}$. Thus, it follows that

$$
\begin{aligned}
\operatorname{Pr}\{(\boldsymbol{x}(k+1), \boldsymbol{N}(k+1))=(F(x, i), l) \mid(\boldsymbol{x}(k), \boldsymbol{N}(k))=(x, i)\} & \\
= & \operatorname{Pr}\{\boldsymbol{N}(k+1)=l \mid(x(k), \boldsymbol{N}(k))=(x, i)\} \\
= & \operatorname{Pr}\{\boldsymbol{N}(k+1)=l \mid N(k)=i\},
\end{aligned}
$$

where the last simplification is explained with an argument similar to that in Theorem B.1.3. That is, since $\{\omega: \boldsymbol{x}(k, \omega)=x\} \in \sigma(\boldsymbol{N}(k-1), \ldots, \boldsymbol{N}(k), \boldsymbol{x}(0))$, the simplification follows from the independence of $x(0)$ and $\{N(k), k \geq 0\}$, and the 
Markov property of the latter. Combining these elements together yields

$$
\mathrm{P}_{(x, N)}((x, i), D)=\sum_{l=0}^{\ell_{N}-1} 1_{D}((F(x, i), l)) p_{i, l}^{N} .
$$

It is easy to show that $\mathrm{P}_{(x, N)}$ fulfills properties (MK-i) and (MK-ii). Furthermore, if $D=B \times C$, where $B \in \mathscr{B}\left(\mathbb{R}^{n}\right)$ and $C \in 2^{\mathcal{I}_{\ell_{N}}}, \mathrm{P}_{(x, N)}$ can be written as

$$
\mathrm{P}_{(x, N)}((x, i), B \times C)=\sum_{l=0}^{\ell_{N}-1} \mathbf{1}_{B}(F(x, i)) \mathbf{1}_{C}(l) p_{i, l}^{N} .
$$

Finally, consider the following lemma which provides an alternative proof for the results in (Morozan 1976). This lemma is also consistent with (p. 23, HernándezLerma \& Lasserre 2003).

Lemma IV.2.1 Consider system (65). If $F_{k}=F$ for all $k \geq 0$ and $\{\boldsymbol{N}(k), k \geq 0\}$ is an i.i.d. process taking values on $\mathcal{I}_{\ell_{N}}$, which is independent of $\boldsymbol{x}(0)$, then $\{\boldsymbol{x}(k), k \geq 0\}$ is a Markov chain in $\mathbb{R}^{n}$ with Markov kernel

$$
\mathrm{P}_{x}(x, B)=\sum_{i=0}^{\ell_{N}-1} \mathbf{1}_{B}(F(x, i)) p_{i}
$$

where $p_{i}=\operatorname{Pr}\{N(k)=i\}$.

Proof: First, observe that if $\{\boldsymbol{N}(k), k \geq 0\}$ is an i.i.d. process, then $p_{i, j}^{N}=p_{j}$. Furthermore, $\{(\boldsymbol{x}(k), \boldsymbol{N}(k)), k \geq 0\}$ is also a Markov chain. Hence, assuming that $\operatorname{Pr}\{\boldsymbol{x}(k+1) \in B \mid \boldsymbol{x}(k)=x\} \neq 0$, it follows that

$$
\begin{aligned}
\operatorname{Pr}\{\boldsymbol{x}(k+1) & \in B \mid \boldsymbol{x}(k)=x\} \\
& =\sum_{i=0}^{\ell_{N}-1} \sum_{j=0}^{\ell_{N}-1} \operatorname{Pr}\{\boldsymbol{x}(k+1) \in B, \boldsymbol{N}(k+1)=j, \boldsymbol{N}(k)=i \mid \boldsymbol{x}(k)=x\} \\
& =\sum_{i=0}^{\ell_{N}-1} \sum_{j=0}^{\ell_{N}-1} \frac{\operatorname{Pr}\{\boldsymbol{x}(k+1) \in B, \boldsymbol{N}(k+1)=j, \boldsymbol{N}(k)=i, \boldsymbol{x}(k)=x\}}{\operatorname{Pr}\{\boldsymbol{x}(k)=x\}}
\end{aligned}
$$




$$
\begin{aligned}
& \left.=\sum_{i=0}^{\ell_{N}-1} \sum_{j=0}^{\ell_{N}-1} \mathrm{P}_{(x, N)}((x, i), B \times\{j\})\right) \frac{\operatorname{Pr}\{\boldsymbol{x}(k)=x, \boldsymbol{N}(k)=i\}}{\operatorname{Pr}\{\boldsymbol{x}(k)=x\}} \\
& =\sum_{i=0}^{\ell_{N}-1} \sum_{j=0}^{\ell_{N}-1} \mathrm{P}_{(x, N)}((x, i), B \times\{j\}) \operatorname{Pr}\{\boldsymbol{N}(k)=i \mid \boldsymbol{x}(k)=x\} .
\end{aligned}
$$

Note from (65) that $\boldsymbol{x}(k)$ is a function of $\left\{\boldsymbol{x}_{0}, \boldsymbol{N}(0), \ldots, \boldsymbol{N}(k-1)\right\}$. This fact and the assumption that $\boldsymbol{x}_{0}$ and $\{\boldsymbol{N}(k), k \geq 0\}$ are independent imply that $\boldsymbol{x}(k)$ and $\boldsymbol{N}(k)$ are independent for every $k \geq 0$. Hence, using (69) in the expression above yields

$$
\begin{aligned}
\operatorname{Pr}\{\boldsymbol{x}(k+1) \in B \mid \boldsymbol{x}(k)=x\} & =\sum_{i=0}^{\ell_{N}-1} \sum_{j=0}^{\ell_{N}-1}\left(\sum_{l=0}^{\ell_{N}-1} \mathbf{1}_{B}(F(x, i)) \mathbf{1}_{\{j\}}(l) p_{i, l}^{N}\right) p_{i} \\
& =\sum_{i=0}^{\ell_{N}-1} \sum_{j=0}^{\ell_{N}-1} \mathbf{1}_{B}(F(x, i)) p_{i, j}^{N} p_{i} \\
& =\sum_{i=0}^{\ell_{N}-1} 1_{B}(F(x, i)) p_{i} \sum_{j=1}^{\ell_{N}-1} p_{i, j}^{N} \\
& =\sum_{i=0}^{\ell_{N}-1} 1_{B}(F(x, i)) p_{i}=\mathrm{P}_{x}(x, B) .
\end{aligned}
$$

It is easy to see that $\mathrm{P}_{x}$ defined above complies with properties (MK-i) and (MK-ii). Thus, it follows from Kolmogorov's Existence Theorem (Shiryaev 1995, Billingsley 1995) that $\{\boldsymbol{x}(k), k \geq 0\}$ is a Markov chain.

The Markov property of a HJLS can now be established using the tools developed in this section. This analysis is presented in the next section together with a discussion of the evolutions of the distributions, $\mu_{k}^{y}$, associated with a HJLS.

\section{IV.3 THE MARKOV PROPERTY OF HYBRID JUMP LINEAR SYSTEMS}

This section is divided into three parts. The first subsection presents a discussion of finite state machines in the framework of Theorem IV.2.1. The goal is to show the 
circumstances under which the state process of a FSM, $\{\boldsymbol{z}(k), k \geq 0\}$, constitutes a Markov chain, and to derive its associated Markov kernel. Although this is an interesting result in its own right, it is included mainly to motivate and clarify the notation in (75) and (77), which is used extensively at the end of this section. The second subsection shows that a canonical HJLS can be posed in the framework of Theorem IV.2.1. This clearly shows then that systems modeled as HJLS's are Markov chains. As a consequence, it possible to derive their associated Markov kernels both when $\{N(k), k \geq 0\}$ is a Markov chain and when it is an i.i.d. process. The last subsection derives closed-form formulas for the probability distribution, $\mu_{k}^{y}$, of a HJLS's state vector, $\boldsymbol{y}(k)$. The derivation is based on the aforementioned Markov kernels and on equation (64).

\section{IV.3.1 Markov Kernels Associated with FSM's}

Recall from Definition II.2.2 that the evolution of the states of a finite state machine with input process $\{\boldsymbol{N}(k), k \geq 0\}$ is given by

$$
\boldsymbol{z}(k+1)=S_{\boldsymbol{N}(k)} \boldsymbol{z}(k)=\sum_{i=0}^{\ell_{N}-1} \boldsymbol{1}_{\{\boldsymbol{N}(k)=i\}} S_{i} \boldsymbol{z}(k)=F_{z}(\boldsymbol{z}(k), N(k)) .
$$

It is clear that the equation above is a particular example of (65), where $n=\ell_{s}$ and $m=1$. Thus, if $\{\boldsymbol{N}(k), k \geq 0\}$ is a Markov chain, it follows that $\{(\boldsymbol{z}(k), \boldsymbol{N}(k)), k \geq$ $0\}$ is also a Markov chain over $\Sigma_{S} \times \mathcal{I}_{\ell_{N}}$, provided that $\boldsymbol{z}(0)$ and $\{\boldsymbol{N}(k), k \geq 0\}$ are independent. This derivation is important because it provides an alternative proof of the well-known fact that the cross product of the state and the input of a FSM constitutes a Markov chain (cf. Patilkulkarni et al. 2004). The focus here, however, is the Markov Kernel associated with $\{(\boldsymbol{z}(k), \boldsymbol{N}(k)), k \geq 0\}$. When $\{\boldsymbol{N}(k), k \geq 0\}$ is 
a Markov chain, it can be shown that the corresponding Markov kernel is given by

$$
\mathrm{P}_{(z, N)}\left(\left(e_{r}, i\right), C \times D\right)=\sum_{l=0}^{\ell_{N}-1} \mathbf{1}_{C}\left(S_{i} e_{r}\right) \mathbf{1}_{D}(l) p_{i, l}^{N}
$$

where $i \in \mathcal{I}_{\ell_{N}}, e_{r} \in \Sigma_{S}, C \in 2^{\Sigma_{S}}$, and $D \in 2^{\mathcal{I}_{\ell_{N}}}$. It follows from Lemma IV.2.1 that $\{\boldsymbol{z}(k), k \geq 0\}$ is a Markov chain over $\Sigma_{S}$ when $\{\boldsymbol{N}(k), k \geq 0\}$ is an i.i.d. process. In which case, it can be shown that its Markov kernel is simply given by

$$
\mathrm{P}_{z}\left(e_{r}, C\right)=\sum_{l=0}^{\ell_{N}-1} \mathbf{1}_{C}\left(S_{l} e_{r}\right) p_{l}
$$

where $p_{l}=\operatorname{Pr}\{N(k)=l\}, e_{r} \in \Sigma_{S}$, and $C \in 2^{\Sigma_{S}}$ (equation (73) can be derived from (72) by setting $D=\mathcal{I}_{\ell_{N}}$ and noting that $p_{l}=p_{i, l}^{N}$ when $\{N(k), k \geq 0\}$ is an i.i.d. process).

Remark IV.3.1 When $\{N(k), k \geq 0\}$ is an i.i.d. process, $\Pi_{z}=\left[p_{i, j}^{z}\right]$, the transition probability matrix associated with the Markov chain $\{\boldsymbol{z}(k), k \geq 0\}$, is given by

$$
\Pi_{z}=S^{\mathbf{T}} \triangleq \sum_{l=0}^{\ell_{N}-1} S_{l}^{\mathrm{T}} p_{l}
$$

To see this, observe from (73) that

$$
p_{i, j}^{z}=\mathrm{P}_{z}\left(e_{i},\left\{e_{j}\right\}\right)=\sum_{l=0}^{\ell_{N}-1} 1_{\left\{e_{j}\right\}}\left(S_{l} e_{i}\right) p_{l}=\sum_{l=0}^{\ell_{N}-1} e_{j}^{\mathrm{T}} S_{l} e_{i} p_{l}=e_{j}^{\mathrm{T}} S e_{i}=e_{i}^{\mathrm{T}} S^{\mathrm{T}} e_{j}
$$

Consequently,

$\Pi_{z}=\left[\begin{array}{ccc}e_{1}^{\mathrm{T}} S^{\mathrm{T}} e_{1} & \ldots & e_{1}^{\mathrm{T}} S^{\mathrm{T}} e_{\ell_{S}} \\ \vdots & \ddots & \vdots \\ e_{\ell_{S}}^{\mathrm{T}} S^{\mathrm{T}} e_{1} & \ldots & e_{\ell_{S}}^{\mathrm{T}} S^{\mathrm{T}} e_{\ell_{S}}\end{array}\right]=\left[\begin{array}{c}e_{1}^{\mathrm{T}} S^{\mathrm{T}} \\ \vdots \\ e_{\ell_{S}}^{\mathrm{T}} S^{\mathrm{T}}\end{array}\right]\left[\begin{array}{lll}e_{1} & \ldots & e_{\ell_{S}}\end{array}\right]=\left[\begin{array}{c}e_{1}^{\mathrm{T}} \\ \vdots \\ e_{\ell_{S}}^{\mathrm{T}}\end{array}\right] S^{T}\left[\begin{array}{lll}e_{1} & \ldots & e_{\ell_{S}}\end{array}\right]$

Finally, note that $I_{\ell_{S}}$, an $\ell_{S} \times \ell_{S}$ identity matrix, can be written as $I_{\ell_{S}}=\left[\begin{array}{lll}e_{1} & \ldots & e_{\ell_{S}}\end{array}\right]$.

Hence, $\Pi_{z}=S^{\mathrm{T}}$. 
Remark IV.3.2 When $\{N(k), k \geq 0\}$ is a Markov chain, a similar approach can be used to compute an expression for $\Pi_{(z, N)}$, the transition probability matrix associated with $\{(\boldsymbol{z}(k), \boldsymbol{N}(k)), k \geq 0\}$. However, a simpler expression can be obtained for the probability matrix of $\{(\boldsymbol{N}(k), \boldsymbol{z}(k)), k \geq 0\}$ as shown in (Patilkulkarni et al. 2004). ${ }^{2}$

\section{On FSM's Embedded in HJLS's}

Consider the state evolution of the FSM embedded in a HJLS. It is given by

$$
\boldsymbol{z}(k+1)=S_{\boldsymbol{N}(k), \psi(\boldsymbol{x}(k))} \boldsymbol{z}(k)
$$

Note that if $\boldsymbol{x}(k)=\bar{x} \in \mathbb{R}^{n}$ for all $k \geq 0$, then the equation above could be rewritten as

$$
\boldsymbol{z}(k+1)=\tilde{S}_{\boldsymbol{N}(k)} \boldsymbol{z}(k),
$$

where $\tilde{S}_{\boldsymbol{N}(k)}=S_{\boldsymbol{N}(k), \psi(\tilde{x})}$. Since this equation is similar to (71), one can define a Markov kernel associate with $\{(\boldsymbol{z}(k), \boldsymbol{N}(k)), k \geq 0\}$ as follows:

$$
\underset{(r, i),(C, D)}{\mathrm{P}}(\bar{x}) \triangleq \sum_{l=0}^{\ell_{N}-1} \mathbf{1}_{C}\left(S_{i, \psi(\bar{x})} e_{r}\right) \mathbf{1}_{D}(l) p_{i, l}^{N},
$$

where $e_{r} \in \Sigma_{S}, i \in \mathcal{I}_{\ell_{N}}, C \in 2^{\Sigma_{S}}, D \in 2^{\mathcal{I}_{\ell_{N}}}$, and $\bar{x} \in \mathbb{R}^{n}$.

Remark IV.3.3 Note that $\underset{(r, i),(C, D)}{\mathrm{P}}(\bar{x})$ can be seen as a family of Markov kernels indexed by $\bar{x}$. Also note that there are only $\ell_{\nu}$ possible Markov kernels, since $\bar{x}$ must belong to one the partitions induced by $\psi$. Finally, observe that the notation, $\underset{(r, i),(C, D)}{\mathrm{P}}(\bar{x})$, is simply a compactification of the notation, $\mathrm{P}\left(\left(e_{r}, i\right), C \times D\right)$.

\footnotetext{
${ }^{2}$ Note that Patilkulkarni et al. study the joint process $\{(N(k), z(k)), k \geq 0\}$ instead of the process $\{(\boldsymbol{z}(k), N(k)), k \geq 0\}$, which is the focus here.
} 
In general, of course, $\boldsymbol{x}(k)$ is not constant for every $k \geq 0$. Therefore, (75) cannot be used to show that $\{(\boldsymbol{z}(k), \boldsymbol{N}(k)), k \geq 0\}$ is a Markov chain for every HJLS. Instead, $\underset{(r, i),(C, D)}{\mathrm{P}}(\bar{x})$ can be interpreted as follows: if it is known that $\boldsymbol{x}(k)=\bar{x}, \boldsymbol{z}(k)=e_{\boldsymbol{r}}$, and $\boldsymbol{N}(k)=i$, observe that the probability that at time $k+1,(z(k+1), N(k+1)) \in C \times D$, for some $C \in 2^{\Sigma_{S}}$ and $D \in 2^{\mathcal{I}_{\ell_{N}}}$ is either equal to zero, if $S_{i, \psi(\bar{x})} e_{r} \notin C$, or equal to $\sum_{j \in D} p_{i, j}^{N} \geq 0$, when $S_{i, \psi(\bar{x})} e_{r} \in C$. That is,

$$
\begin{aligned}
\operatorname{Pr}\left\{\boldsymbol{z}(k+1) \in C, \boldsymbol{N}(k+1) \in D \mid \boldsymbol{z}(k)=e_{r}, \boldsymbol{N}(k)=\right. & i, \boldsymbol{x}(k)=\bar{x}\} \\
& =\sum_{l=0}^{\ell_{N}-1} \mathbf{1}_{C}\left(S_{i, \psi(\bar{x})} e_{r}\right) \mathbf{1}_{D}(l) p_{i, l}^{N} .
\end{aligned}
$$

This clearly suggests that

$$
\underset{(r, i),(C, D)}{\mathrm{P}}(\bar{x})=\operatorname{Pr}\left\{\boldsymbol{z}(k+1) \in C, \boldsymbol{N}(k+1) \in D \mid \boldsymbol{z}(k)=e_{r}, \boldsymbol{N}(k)=i, \boldsymbol{x}(k)=\bar{x}\right\}
$$

This assertion is proven in the next subsection. A similar development is possible when $\{\boldsymbol{N}(k), k \geq 0\}$ is an i.i.d. process. In this case, define $\underset{r, C}{\mathrm{P}}(\bar{x})$ as

$$
\underset{r, C}{\mathrm{P}}(\bar{x}) \triangleq \sum_{i=0}^{\ell_{N}-1} \mathbf{1}_{C}\left(S_{i, \psi(\bar{x})} e_{r}\right) p_{i}
$$

where $e_{r} \in \Sigma_{S}, C \in 2^{\Sigma_{S}}$, and $\bar{x} \in \mathbb{R}^{n}$. A reasoning similar to the one above suggests that $\underset{r, C}{\mathrm{P}}(\bar{x})$ can be interpreted as follows (see next subsection for a proof)

$$
\underset{r, C}{\mathrm{P}}(\bar{x})=\operatorname{Pr}\left\{\boldsymbol{z}(k+1) \in C \mid \boldsymbol{z}(k)=e_{r}, \boldsymbol{x}(k)=\bar{x}\right\}
$$

Note that a direct comparison with (72) and (73) reveals that $\underset{(r, i),(C, D)}{\mathrm{P}}(\bar{x})$ and $\underset{r, C}{\mathrm{P}}(\bar{x})$ in, respectively, (75) and (77) are both Markov kernels for every fixed $\bar{x} \in \mathbb{R}^{n}$. On the other hand, the equalities in (76) and (78) need to be formally established. This 
will be accomplished in the next subsection, after introducing the Markov kernels associated with HJLS's. As a final remark, consider the following simplifying notation. If $C=\left\{e_{s}\right\}$ and $D=\{j\}$ then the following abbreviated notation will be used

$$
\begin{gathered}
\underset{(r, i),(s, j)}{\mathrm{P}}(\bar{x}) \triangleq \underset{(r, i),\left(\left\{e_{s}\right\},\{j\}\right)}{\mathrm{P}}(\bar{x})=\mathbf{1}_{\left\{e_{s}\right\}}\left(S_{i, \psi(\bar{x})} e_{r}\right) p_{i, j}^{N} \\
\underset{r, s}{\mathrm{P}}(\bar{x}) \triangleq \underset{r,\left\{e_{s}\right\}}{\mathrm{P}}(\bar{x})=\sum_{i=0}^{\ell_{N}-1} \mathbf{1}_{\left\{e_{s}\right\}}\left(S_{i, \psi(\bar{x})} e_{r}\right) p_{i} .
\end{gathered}
$$

\section{IV.3.2 On the Markov Property and Markov Kernel of Canonical HJLS's}

Recall from the discussion in Chapter II that every HJLS with an embedded Moore-type FSM can be transformed into an equivalent canonical HJLS. Consequently, the analysis that follows focuses on determining the Markov property and Markov kernel of the latter. Recall that the evolution equation of a canonical HJLS given in Definition II.2.4 can be written as

$$
\left[\begin{array}{c}
\boldsymbol{x}(k+1) \\
\boldsymbol{z}(k+1)
\end{array}\right]=\left[\begin{array}{c}
A_{\boldsymbol{z}(k)} \boldsymbol{x}(k) \\
S_{\boldsymbol{N}(k), \psi(\boldsymbol{x}(k))} \boldsymbol{z}(k)
\end{array}\right],
$$

where

$$
\psi(x)=\sum_{i=0}^{\ell_{\nu}-1} i 1_{\left\{x \in R_{i}\right\}}, \quad \bigcup_{i \in \mathcal{I}_{\ell_{\nu}}} R_{i}=\mathbb{R}^{n}
$$

As before, let $Y=\mathbb{R}^{n} \times \Sigma_{S}, \boldsymbol{y}(k)=\left[\boldsymbol{x}(k)^{\mathrm{T}} \boldsymbol{z}(k)^{\mathrm{T}}\right]^{\mathrm{T}}$ represent the state of the HJLS, and define $F: Y \times \mathcal{I}_{\ell_{N}} \mapsto Y$ as

$$
F(x, z, l) \triangleq\left[\begin{array}{c}
F_{1}(x, z) \\
F_{2}(x, z, l)
\end{array}\right]=\left[\begin{array}{c}
A_{z} x \\
S_{l, \psi(x)} z
\end{array}\right]=\left[\begin{array}{c}
\sum_{i=1}^{\ell_{S}} A_{i} x \mathbf{1}_{\left\{z=e_{i}\right\}} \\
\sum_{i=0}^{\ell_{N}-1} \sum_{j=0}^{\ell_{\nu}-1} S_{i, j} z \mathbf{1}_{\{l \in\{i\}\}} \mathbf{1}_{\left\{x \in R_{j}\right\}}
\end{array}\right]
$$


Note that (80) can be expressed as

$$
\boldsymbol{y}(k+1)=F(\boldsymbol{y}(k), \boldsymbol{N}(k))
$$

where $F$ in (82) is a Borel function. Moreover, observe that $\boldsymbol{y}(k) \in Y \subset \mathbb{R}^{n+\ell_{S}}$. Thus, (83) is a particular case of (65) in which $n$ has been replaced by $n+\ell_{S}$ and $m=1$ $\left(\boldsymbol{N}(k) \in \mathcal{I}_{\ell_{N}} \subset \mathbb{R}^{1}\right)$. This establishes the Markov property of $\{(\boldsymbol{y}(k), \boldsymbol{N}(k)), k \geq 0\}$ provided that $\boldsymbol{y}(0)$ is integrable and independent of the Markov chain $\{\boldsymbol{N}(k), k \geq 0\}$. Consequently, this and the following additional assumptions will be taken to hold in the sequel.

Assumption IV.3.1 Assume that $\boldsymbol{y}(0)$ and $\{\boldsymbol{N}(k), k \geq 0\}$ are independent. It is also assumed that $\boldsymbol{z}(0)$ and $\boldsymbol{x}(0)$ are independent. Let the distribution of $\boldsymbol{x}(0)$ be $\mu_{0}^{x}=\delta_{\bar{x}}(B)$, for some $\bar{x} \in \mathbb{R}^{n}$ and every $B \in \mathscr{B}\left(\mathbb{R}^{n}\right)\left(\delta_{\bar{x}}(\cdot)\right.$ is the Dirac measure $)$

Under Assumption IV.3.1, $\{(\boldsymbol{y}(k), \boldsymbol{N}(k)), k \geq 0\}$ is a Markov chain on $Y \times \mathcal{I}_{\ell_{N}}$. Consequently, it follows from (69) that its kernel, $\mathrm{P}_{(y, N)}$, is given by

$$
\mathrm{P}_{(y, N)}\left(\left(\left[\begin{array}{c}
x \\
e_{r}
\end{array}\right], i\right), B \times C \times D\right)=\sum_{l=0}^{\ell_{N}-1} \mathbf{1}_{B}\left(A_{e_{r}} x\right) \mathbf{1}_{C}\left(S_{i, \psi(x)} e_{r}\right) \mathbf{1}_{D}(l) p_{i, l}^{N},
$$

where $x \in \mathbb{R}^{n}, e_{r} \in \Sigma_{s}, i \in \mathcal{I}_{\ell_{N}}, B \in \mathscr{B}\left(\mathbb{R}^{n}\right), C \in 2^{\Sigma_{S}}$, and $D \in 2^{\mathcal{I}_{\ell_{N}}}$. Observe from (75) that (84) can be written as

$$
\mathrm{P}_{(y, N)}\left(\left(\left[\begin{array}{c}
x \\
e_{r}
\end{array}\right], i\right), B \times C \times D\right)=\mathbf{1}_{B}\left(A_{e_{r}} x\right) \underset{\left(e_{r}, i\right),(C, D)}{\mathrm{P}}(x) .
$$

Moreover, if follows from the discussion on Markov kernels associated with a Markov chain provided in Subsection IV.1.1 (see equation (61)) that

$$
\mathrm{P}_{(y, N)}\left(\left(\left[\begin{array}{c}
x \\
e_{r}
\end{array}\right], i\right), \mathbb{R}^{n} \times C \times D\right)
$$




$$
\begin{aligned}
& =\operatorname{Pr}\left\{\boldsymbol{y}(k+1) \in \mathbb{R}^{n} \times C, \boldsymbol{N}(k+1) \in D \mid \boldsymbol{y}(k)=\left[\begin{array}{c}
x \\
e_{r}
\end{array}\right], \boldsymbol{N}(k)=i\right\} \\
& =\operatorname{Pr}\left\{\boldsymbol{z}(k+1) \in C, \boldsymbol{N}(k+1) \in D \mid \boldsymbol{z}(k)=e_{r}, \boldsymbol{N}(k)=i, \boldsymbol{x}(k)=x\right\} \\
& \left.=\operatorname{(en}_{r}, i\right),(C, D) \\
& \mathrm{P}
\end{aligned}
$$

Clearly, the last two equalities establish (76). A similar derivation is possible when $\{\boldsymbol{N}(k), k \geq 0\}$ is an i.i.d. process. In such cases, Lemma IV.2.1 implies that $\{\boldsymbol{y}(k), k \geq 0\}$ is a Markov chain in $Y$ with Markov kernel given by

$$
\mathrm{P}_{y}\left(\left[\begin{array}{l}
x \\
e_{r}
\end{array}\right], B \times C\right)=\sum_{i=0}^{\ell_{N}-1} \mathbf{1}_{B}\left(A_{e_{r}} x\right) \mathbf{1}_{C}\left(S_{i, \psi(x)} z\right) p_{i} .
$$

As before, observe that

$$
\mathrm{P}_{y}\left(\left[\begin{array}{l}
x \\
e_{r}
\end{array}\right], B \times C\right)=\mathbf{1}_{B}\left(A_{e_{r}} x\right) \underset{r, C}{\mathrm{P}}(x)
$$

which, after taking $B=\mathbb{R}^{n}$ and using (61), proves (78). Equations (84) and (85) will be instrumental in studying the probability distributions of $\{\boldsymbol{y}(k), k \geq 0\}$ as done in the next section.

\section{IV.3.3 Probability Distributions Associated with HJLS's}

The goal of this last subsection is to derive closed-form expressions for the probability distributions $\mu_{k}^{y}$ associated with the state vector of a HJLS when $\{\boldsymbol{N}(k), k \geq 0\}$ is either an i.i.d. process or a Markov chain. In the latter case, the probability distribution, $\mu_{k}^{(y, N)}$, of $\{(\boldsymbol{y}(k), N(k)), k \geq 0\}$ is computed first and then used to derive $\mu_{k}^{y}$. These probability distributions are defined as in subsection IV.1.1, that is, $\mu_{k}^{y}(B)=\operatorname{Pr}\{\boldsymbol{y}(k) \in B\}$ and $\mu_{k}^{(y, N)}(C)=\operatorname{Pr}\{(\boldsymbol{y}(k), \boldsymbol{N}(k)) \in C\}$, for every $k \geq 0$, $B \in \mathscr{B}(Y)$, and $C \in \mathscr{B}(Y) \otimes 2^{\mathcal{I}_{\ell_{N}}}$. As done earlier, the discussion will be restricted to canonical HJLS. 


\section{Probability Distribution of a Canonical HJLS's with i.i.d. Inputs}

Under Assumption IV.3.1, the probability distribution of $\boldsymbol{y}(0), \mu_{0}^{y}$, has the form $\mu_{0}^{y}(B \times C)=\delta_{\bar{x}}(B) \mu_{0}^{z}(C)$, for any $B \in \mathscr{B}\left(\mathbb{R}^{n}\right)$ and $C \in 2^{\Sigma_{S}}$. It follows from the Kolmogorov equation (62) and the definition of $F$ in (82) that

$$
\begin{aligned}
\mu_{1}^{y}(B \times C) & =\mathrm{P}_{y} \mu_{0}^{y}(B \times C) \\
& =\int_{Y} \mathrm{P}_{y}(y, B \times C) \mu_{0}^{y}(d y) \\
& =\int_{Y}^{\ell_{N}-1} \sum_{i=0}^{\mathbf{1}_{B}\left(F_{1}(y)\right) \mathbf{1}_{C}\left(F_{2}(y, i)\right) p_{i} \mu_{0}^{y}(d y) .}
\end{aligned}
$$

Using Fubini's Theorem (Billingsley 1995), the expression above can be simplified as follows:

$$
\begin{aligned}
\mu_{1}^{y}(B \times C) & =\sum_{i=0}^{\ell_{N}-1} \int_{\Sigma_{S}} \int_{\mathbb{R}^{n}} \mathbf{1}_{B}\left(A_{z} x\right) \mathbf{1}_{C}\left(S_{i, \psi(x)} z\right) p_{i} \delta_{\bar{x}}(d x) \mu_{0}^{z}(d z) \\
& =\sum_{i=0}^{\ell_{N}-1} \int_{\Sigma_{S}} \mathbf{1}_{B}\left(A_{z} \bar{x}\right) \mathbf{1}_{C}\left(S_{i, \psi(\bar{x})} z\right) p_{i} \mu_{0}^{z}(d z) \\
& =\sum_{r_{0}=1}^{\ell_{S}} \mu_{0}^{z}\left(\left\{e_{r_{0}}\right\}\right) \mathbf{1}_{B}\left(A_{e_{r_{0}}} \bar{x}\right) \sum_{i=0}^{\ell_{N}-1} \mathbf{1}_{C}\left(S_{i, \psi(\bar{x})} e_{r_{0}}\right) p_{i}
\end{aligned}
$$

Observe that the right-most summation in the expression above is $\underset{r_{0}, C}{\mathrm{P}}(\bar{x})$. Also note that $\mathbf{1}_{B}\left(A_{e_{r_{0}}} \bar{x}\right)=\delta_{A_{e_{0}} \bar{x}}(B)$. Thus, it follows that

$$
\mu_{1}^{y}(B \times C)=\sum_{r_{0}=1}^{\ell_{S}} \mu_{0}^{z}\left(\left\{e_{r_{0}}\right\}\right) \delta_{A_{e_{0}} \bar{x}}(B) \underset{r_{0}, C}{\mathrm{P}}(\bar{x}) .
$$

Recall that for every fixed $\bar{x}, \underset{r_{0}, C}{\mathrm{P}}(\bar{x})$ is a Markov kernel. Thus, for every $e_{r_{0}} \in \Sigma_{S}$, $\underset{r_{0},(\cdot)}{\mathrm{P}}(\bar{x})$ is a probability measure over $\Sigma_{S}$. Let $\mu_{\bar{x}, r_{0}}(B \times C) \triangleq \delta_{A_{e_{0}} \bar{x}}(B) \underset{r_{0}, C}{\mathrm{P}}(\bar{x})$ for every $B \in \mathscr{B}\left(\mathbb{R}^{n}\right), C \in 2^{\Sigma_{S}}$, and observe that this is a product measure over $Y$, which has the same form as $\mu_{0}^{y}$. Thus, $\mathrm{P}_{y} \mu_{\bar{x}, r_{0}}(B \times C)$ can be computed using the 
same procedure as in (86). That is,

$$
\mathrm{P}_{y} \mu_{\bar{x}, r_{0}}(B \times C)=\sum_{r_{1}=1}^{\ell_{S}} \underset{r_{0}, r_{1}}{\mathrm{P}}(\bar{x}) \delta_{A_{e_{r_{1}}} A_{e_{r_{0}}} \bar{x}}(B) \underset{r_{1}, C}{\mathrm{P}}\left(A_{e_{r_{0}}} \bar{x}\right) .
$$

Furthermore, since $\mu_{2}^{y}(B \times C)=\mathrm{P}_{y} \mu_{1}^{y}(B \times C)$, it follows from the expression above and from (86) that

$$
\begin{aligned}
\mu_{2}^{y}(B \times C) & =\mathrm{P} \mu_{1}^{y}(B \times C) \\
& =\sum_{r_{0}=1}^{\ell_{S}} \mu_{0}^{z}\left(\left\{e_{r_{0}}\right\}\right) \mathrm{P}_{y} \mu_{r_{0}}^{\bar{x}}(B \times C) \\
& =\sum_{r_{0}=1}^{\ell_{S}} \sum_{r_{1}=1}^{\ell_{S}} \mu_{0}^{z}\left(\left\{e_{r_{0}}\right\}\right) \underset{r_{0}, r_{1}}{\mathrm{P}}(\bar{x}) \delta_{A_{e_{r_{1}}} A_{e_{r_{0}}} \bar{x}}(B) \underset{r_{1}, C}{\mathrm{P}}\left(A_{e_{r_{0}}} \bar{x}\right) .
\end{aligned}
$$

Again, letting $\mu_{\bar{x}, r_{0} r_{1}}(B \times C) \triangleq \delta_{A_{e_{r_{1}}} A_{e_{r_{0}}}}(B) \underset{r_{1}, C}{\mathrm{P}}\left(A_{e_{r_{0}}} \bar{x}\right)$, yields, after computing $P_{y} \mu_{\bar{x}, r_{0} r_{1}}(B \times C)$ and repeating the procedure above,

$$
\begin{aligned}
\mu_{3}^{y}(B \times C) & \\
& =\sum_{r_{0}=1}^{\ell_{S}} \sum_{r_{1}=1}^{\ell_{S}} \sum_{r_{2}=1}^{\ell_{S}} \mu_{0}^{z}\left(\left\{e_{r_{0}}\right\}\right) \underset{r_{0}, r_{1}}{\mathrm{P}}(\bar{x}) \underset{r_{1}, r_{2}}{\mathrm{P}}\left(A_{e_{r_{0}}} \bar{x}\right) \delta_{A_{e_{r_{2}}} A_{e_{r_{1}}} A_{e_{r_{0}}} \bar{x}}(B) \underset{r_{2}, C}{\mathrm{P}}\left(A_{e_{r_{1}}} A_{e_{r_{0}}} \bar{x}\right) .
\end{aligned}
$$

Hence, a simple induction argument proves the following theorem.

Theorem IV.3.1 Consider a canonical HJLS with state evolution equation (80). Let the input process $\{N(k), k \geq 0\}$ be an i.i.d. process, and let Assumption IV.3.1 hold. The distribution of $\boldsymbol{y}(k)=\left[\boldsymbol{x}(k)^{\mathrm{T}} \boldsymbol{z}(k)^{\mathrm{T}}\right]^{\mathrm{T}}, \mu_{k}^{y}$, is given by

$$
\begin{aligned}
& \mu_{k}^{y}(B \times C)= \\
& \sum_{r_{0}=1}^{\ell_{S}} \cdots \sum_{r_{k-1}=1}^{\ell_{S}} \mu_{0}^{z}\left(\left\{e_{r_{0}}\right\}\right) \underset{r_{0}, r_{1}}{\mathrm{P}}(\bar{x}) \underset{r_{1}, r_{2}}{\mathrm{P}}\left(A_{e_{r_{0}}} \bar{x}\right) \cdots{ }_{r_{k-2}, r_{k-1}}^{\mathrm{P}}\left(A_{e_{r_{k-3}}} \cdots A_{e_{r_{0}}} \bar{x}\right) . \\
& \underset{r_{k-1}, C}{\mathrm{P}}\left(A_{e_{r_{k-2}}} \cdots A_{e_{r_{0}}} \bar{x}\right) \delta_{A_{e_{r_{k-1}}} \cdots A_{e_{r_{0}}} \bar{x}}(B),
\end{aligned}
$$

for any $B \in \mathscr{B}\left(\mathbb{R}^{n}\right), C \in 2^{\Sigma_{s}}$, and $k \geq 1$. 
Proof: A proof by induction is immediate using the procedure followed above to derive (87) from (86).

\section{Probability Distribution of a Canonical HJLS with Markovian Input}

A procedure similar to the one that lead to Theorem IV.3.1 can be followed when $\{\boldsymbol{N}(k), k \geq 0\}$ is a Markov chain. In this case, however, the procedure yields a closedform expression for $\mu_{k}^{(y, N)}$. Thus, extra steps are needed to compute $\mu_{k}^{y}$ from $\mu_{k}^{(y, N)}$. As before, Assumption IV.3.1 implies that $\mu_{0}^{(y, N)}(B \times C \times D)=\delta_{\bar{x}}(B) \mu_{0}^{(z, N)}(C \times D)$, for any $B \in \mathscr{B}\left(\mathbb{R}^{n}\right), C \in 2^{\Sigma_{S}}$, and $D \in 2^{\mathcal{I}_{\ell_{N}}}$, where $\mu_{0}^{(z, N)}$ is any distribution over $\Sigma_{S} \times \mathcal{I}_{\ell_{N}}$. (Assumption IV.3.1 also requires that $\mu_{0}^{(z, N)}=\mu_{0}^{z} \mu_{0}^{N}$, but this is not relevant for the subsequent analysis). Using the Kolmogorov equation (62) and the definition of $F$ in (82) yields

$$
\begin{aligned}
\mu_{1}^{(y, N)}(B \times C \times D) & =\mathrm{P}_{(y, N)} \mu_{0}^{(y, N)}(B \times C \times D) \\
& =\int_{Y \times \mathcal{I}_{\ell_{N}}} \mathrm{P}_{(y, N)}(y, i, B \times C) \mu_{0}^{(y, N)}(d y \times d i) \\
& =\int_{Y \times \mathcal{I}_{\ell_{N}}} \sum_{j=0}^{\ell_{N}-1} \mathbf{1}_{B}\left(F_{1}(y)\right) \mathbf{1}_{C}\left(F_{2}(y, i)\right) \mathbf{1}_{D}(j) p_{i, j}^{N} \mu_{0}^{(y, N)}(d y \times d i),
\end{aligned}
$$

where the last expression follows from (84). Again, applying Fubini's Theorem yields

$$
\begin{aligned}
\mu_{1}^{(y, N)}(B \times C \times D) & =\int_{\Sigma_{S} \times \mathcal{I}_{\ell_{N}}} \int_{\mathbb{R}^{n}} \sum_{j=0}^{\ell_{N}-1} \mathbf{1}_{B}\left(A_{z} x\right) \mathbf{1}_{C}\left(S_{i, \psi(x)} z\right) \mathbf{1}_{D}(j) p_{i, j}^{N} \delta_{\bar{x}}(d x) \mu_{0}^{(z, N)}(d z \times d i) \\
& =\int_{\Sigma_{S} \times \mathcal{I}_{\ell_{N}}} \sum_{j=0}^{\ell_{N}-1} \mathbf{1}_{B}\left(A_{z} \bar{x}\right) \mathbf{1}_{C}\left(S_{i, \psi(\bar{x})} z\right) \mathbf{1}_{D}(j) p_{i, j}^{N} \mu_{0}^{(z, N)}(d z \times d i) \\
& =\sum_{r_{0}=1}^{\ell_{S}} \sum_{i_{0}=0}^{\ell_{N}-1} \mu_{0}^{(z, N)}\left(\left\{\left(e_{r_{0}}, i_{0}\right)\right\}\right) \mathbf{1}_{B}\left(A_{e_{r_{0}}} \bar{x}\right) \sum_{j=0}^{\ell_{N}-1} \mathbf{1}_{C}\left(S_{i_{0}, \psi(\bar{x})} e_{r_{0}}\right) \mathbf{1}_{D}(j) p_{i_{0} j}^{N}
\end{aligned}
$$




$$
=\sum_{r_{0}=1}^{\ell_{S}} \sum_{i_{0}=0}^{\ell_{N}-1} \mu_{0}^{(z, N)}\left(\left\{\left(e_{r_{0}}, i_{0}\right)\right\}\right) \delta_{A_{e_{0}} \bar{x}}(B) \underset{\left(r_{0}, i_{0}\right),(C, D)}{\mathrm{P}}(\bar{x}) .
$$

Note that $\underset{\left(r_{0}, i_{0}\right),(C, D)}{\mathrm{P}}(\bar{x})$ is a Markov kernel. Thus, $\underset{\left(r_{0}, i_{0}\right),(\cdot, \cdot)}{\mathrm{P}}(\bar{x})$ is a probability measure on $\Sigma_{S} \times \mathcal{I}_{\ell_{N}}$, which implies that $\mu_{r_{0}, i_{0}}^{\bar{x}}(B \times C \times D) \triangleq \delta_{A_{e_{r_{0}}} \bar{x}}(B) \underset{\left(r_{0}, i_{0}\right),(C, D)}{\mathrm{P}}(\bar{x})$ is a product measure on $Y \times \mathcal{I}_{\ell_{N}}$ with the same characteristics as $\mu_{0}^{(y, N)}$. Hence, $\mathrm{P}_{(y, N)} \mu_{r_{0}, i_{0}}^{\bar{x}}(B \times$ $C \times D$ ) can be computed using the same procedure used to obtain $\mu_{1}^{(y, N)}$. This, in turn, implies that $\mu_{2}^{(y, N)}=\mathrm{P}_{(y, N)} \mu_{1}^{(y, N)}$ can be computed as follows:

$$
\begin{aligned}
& \mu_{2}^{(y, N)}(B \times C \times D) \\
& =\sum_{r_{0}=1}^{\ell_{S}} \sum_{i_{0}=0}^{\ell_{N}-1} \sum_{r_{1}=1}^{\ell_{S}} \sum_{i_{1}=0}^{\ell_{N}-1} \mu_{0}^{(z, N)}\left(\left\{\left(e_{r_{0}}, i_{0}\right)\right\}\right) \underset{\left(r_{0}, i_{0}\right),\left(r_{1}, i_{1}\right)}{\mathrm{P}}(\bar{x}) \delta_{A_{e_{r_{1}}} A_{e_{r_{0}} \bar{x}} \bar{x}}(B) \underset{\left(r_{1}, i_{1}\right),(C, D)}{\mathrm{P}}\left(A_{e_{r_{0}}} \bar{x}\right) .
\end{aligned}
$$

It is clear that, by repeating the same procedure, it is possible to compute $\mu_{3}^{(y, N)}$, $\mu_{4}^{(y, N)}, \ldots$, etc. This is formalized in the next theorem.

Theorem IV.3.2 Consider a canonical HJLS with state evolution equation (80). Let the input process $\{N(k), k \geq 0\}$ be a Markov chain and let Assumption IV.3.1 hold. The distribution of $(\boldsymbol{y}(k), \boldsymbol{N}(k)), \mu_{k}^{(y, N)}$, is given by

$$
\begin{aligned}
& \mu_{k}^{(y, N)}(B \times C \times D)= \\
& \sum_{r_{0}=1}^{\ell_{S}} \sum_{i_{0}=0}^{\ell_{N}-1} \cdots \sum_{r_{k-1}=1}^{\ell_{S}} \sum_{i_{k-1}=0}^{\ell_{N}-1} \mu_{0}^{(z, N)}\left(\left\{\left(e_{r_{0}}, i_{0}\right)\right\}\right) \underset{\left(r_{0}, i_{0}\right),\left(r_{1}, i_{1}\right)}{\mathrm{P}}(\bar{x}) \underset{\left(r_{1}, i_{1}\right),\left(r_{2}, i_{2}\right)}{\mathrm{P}}\left(A_{e_{r_{0}}} \bar{x}\right) \cdots \\
& \underset{\left(r_{k-2}, i_{k-2}\right),\left(r_{k-1}, i_{k-1}\right)}{\mathrm{P}}\left(A_{e_{r_{k-3}}} \cdots A_{e_{r_{0}}} \bar{x}\right) \underset{\left(r_{k-1}, i_{k-1}\right),(C, D)}{\mathrm{P}}\left(A_{e_{r_{k-2}}} \cdots A_{e_{r_{0}}} \bar{x}\right) \delta_{A_{e_{r_{k-1}}} \cdots A_{e_{r_{0}}} \bar{x}}(B)
\end{aligned}
$$

and the distribution of $\boldsymbol{y}(k)$ is given by

$$
\mu_{k}^{y}(B \times C)=\mu_{k}^{(y, N)}\left(B \times C \times \mathcal{I}_{\ell_{N}}\right)
$$

for any $B \in \mathscr{B}\left(\mathbb{R}^{n}\right), C \in 2^{\Sigma_{S}}, D \in 2^{\mathcal{I}_{\ell_{N}}}$ and $k \geq 1$. 
Proof: The proof of (89) follows directly from an induction argument. The proof of (90) follows from the definition of a marginal distribution.

The next section takes advantage of Theorems IV.3.1 and IV.3.2 to derive two new sets of testable sufficient mean square stability tests for HJLS's.

\section{IV.4 SUFFICIENT MS STABILITY TESTS FOR CANONICAL HJLS's}

This section introduces two new sets of sufficient mean square stability tests for canonical HJLS's. The first set of tests is based on imposing a structural restriction on the $A / S$ map. The second set adapts Theorem III.5.2 to HJLS's. This involves computing appropriate upper bound matrices, $M_{\theta}$, and using the concept of A-equivalence explained in the sequel.

\section{IV.4.1 MS Stability Tests Based on Common Invariant Subspaces}

Consider a canonical HJLS with state evolution equation (80). For the JLS embedded in the HJLS define a common invariant subspace as follows.

Definition IV.4.1 $A$ set $\mathfrak{I} \subseteq \mathbb{R}^{n}$ is said to be a common invariant subspace of the jump linear system in (80), or simply a common invariant subspace, if for every $x \in \mathfrak{I}$ it follows that $A_{e_{i}} x \in \mathfrak{I}$ for all $e_{i} \in \Sigma_{S}$.

Examples of common invariant subspaces are $\mathfrak{I}=\mathbb{R}^{n}$ and $\mathfrak{I}=\{0\}$. A less trivial example is the subspace $\mathfrak{I}=\{\alpha p, \forall \alpha \in \mathbb{R}\}$, where $p$ is an eigenvector shared by all $A_{e_{i}}, e_{i} \in \Sigma_{S}$. Clearly, if for a common invariant subspace $\mathfrak{I}, \boldsymbol{x}\left(k^{\prime}\right) \in \mathfrak{I}$ for some 
$k^{\prime} \geq 0$, then $\boldsymbol{x}(k) \in \mathfrak{I}$ for every $k \geq k^{\prime}$. Recall that

$$
\psi(x)=\sum_{i=0}^{\ell_{\nu}-1} i \mathbf{1}_{\left\{x \in R_{i}\right\}}, \quad \bigcup_{i \in \mathcal{I}_{\ell_{\nu}}} R_{i}=\mathbb{R}^{n},
$$

and assume that for some $l \in \mathcal{I}_{\ell_{\nu}}, R_{l}$ is a common invariant subspace. Further assume that $\boldsymbol{x}(0)=\bar{x} \in R_{l}$. Under these conditions, $\psi(\boldsymbol{x}(k))=l$ for all $k \geq 0$, that is, the output of the $A / S$ map is a constant for all $k \geq 0$. This implies that the FSM embedded in the HJLS is "only" driven by the Markov input $\{\boldsymbol{N}(k), k \geq 0\}$. Consequently, the HJLS behaves as a jump linear system driven by a FSM without state feedback, and its stability can be determined with a simple spectral radius test. These ideas are formalized in Theorem IV.4.1, which is based on the following two lemmas. To simplify the discussion, $\mathbb{R}^{n} \backslash \psi$ will denote the partition of $\mathbb{R}^{n}$ induced by $\psi$

Lemma IV.4.1 Consider a canonical HJLS with state evolution equation (80). Let Assumption IV.3.1 hold, and assume that for some $l \in \mathcal{I}_{\ell_{\nu}}, R_{l} \in \mathbb{R}^{n} \backslash \psi$ is a common invariant subspace. Let $\boldsymbol{x}(0)=\bar{x}_{l} \in R_{l}$. If $\{\boldsymbol{N}(k), k \geq 0\}$ is an i.i.d. process, then $\{z(k), k \geq 0\}$ is a Markov chain with initial distribution $\mu_{0}^{z}$ and transition probability $\operatorname{matrix} \Pi_{z}=\left[p_{r s}^{z}\right]$ with

$$
p_{r s}^{z} \triangleq \mathrm{P}_{r, s}\left(\bar{x}_{l}\right)=\sum_{i=0}^{\ell_{N}-1} \mathbf{1}_{\left\{e_{s}\right\}}\left(S_{i, l} e_{r}\right) p_{i} .
$$

Proof: First, observe that $\mu_{k}^{y}$ is given by (88) as a consequence of Theorem IV.3.1. Moreover, since $\bar{x} \in R_{l}$, each term of the form $\underset{k-1, k}{\mathrm{P}}\left(A_{e_{r_{k-2}}} \cdots A_{e_{r_{0}}} \bar{x}\right)$ in (88) can be 
simplified as follows:

$$
\begin{aligned}
\underset{k-1, k}{\mathrm{P}}\left(A_{e_{r_{k-2}}} \cdots A_{e_{r_{0}}} \bar{x}\right) & =\sum_{i=0}^{\ell_{N}-1} \mathbf{1}_{\left\{e_{r_{k}}\right\}}\left(S_{i, \psi\left(A_{e_{r_{k-2}}} \cdots A_{e_{r_{0}} \bar{x}} \bar{x}\right.} e_{r_{k-1}}\right) p_{i} \\
& =\sum_{i=0}^{\ell_{N}-1} \mathbf{1}_{\left\{e_{r_{k}}\right\}}\left(S_{i, l} e_{r_{k-1}}\right) p_{i} \\
& ={ }_{k-1, k}^{\mathrm{P}}(0)=p_{k-1 k}^{z} .
\end{aligned}
$$

Therefore, (88) can be rewritten as

$$
\begin{aligned}
& \mu_{k}^{y}(B \times C)= \\
& \sum_{r_{0}=1}^{\ell_{S}} \cdots \sum_{r_{k-1}=1}^{\ell_{S}} \mu_{0}^{z}\left(\left\{e_{r_{0}}\right\}\right) p_{r_{0} r_{1}}^{z} p_{r_{1} r_{2}}^{z} \cdots p_{r_{k-2} r_{k-1}}^{z} \underset{r_{k-1}, C}{\mathrm{P}}\left(A_{e_{r_{k-2}}} \cdots A_{e_{r_{1}}} \bar{x}\right) \delta_{A_{e_{r_{k-1}}} \cdots A_{e_{0}} \bar{x}}(B) .
\end{aligned}
$$

Note that $\mu_{k}^{z}\left(\left\{e_{r_{k}}\right\}\right)=\mu_{k}^{y}\left(\mathbb{R}^{n} \times\left\{e_{r_{k}}\right\}\right)$, so it follows that

$$
\begin{aligned}
& \mu_{k}^{z}\left(\left\{e_{r_{k}}\right\}\right) \\
& =\sum_{r_{0}=1}^{\ell_{S}} \cdots \sum_{r_{k-1}=1}^{\ell_{S}} \mu_{0}^{z}\left(\left\{e_{r_{0}}\right\}\right) p_{r_{0} r_{1}}^{z} p_{r_{1} r_{2}}^{z} \cdots p_{r_{k-2} r_{k-1}}^{z} \underset{r_{k-1},\left\{e_{r_{k}}\right\}}{\mathrm{P}}\left(A_{e_{r_{k-2}}} \cdots A_{e_{r_{1}}} \bar{x}\right) \delta_{A_{e_{r_{k-1}}} \cdots A_{e_{0}} \bar{x}}\left(\mathbb{R}^{n}\right) \\
& =\sum_{r_{0}=1}^{\ell_{S}} \cdots \sum_{r_{k-1}=1}^{\ell_{S}} \mu_{0}^{z}\left(\left\{e_{r_{0}}\right\}\right) p_{r_{0} r_{1}}^{z} p_{r_{1} r_{2}}^{z} \cdots p_{r_{k-2} r_{k-1}}^{z} p_{r_{k-1} r_{k}}^{z} .
\end{aligned}
$$

Next, observe that $\mu_{k}^{z}\left(\left\{e_{r_{k}}\right\}\right)=\left[\mu_{k}^{z}\left(\left\{e_{1}\right\}\right), \ldots, \mu_{k}^{z}\left(\left\{e_{\ell_{s}}\right\}\right)\right] e_{r_{k}}$. Hence, after a few simplifying steps, the expression above can be rewritten as

$$
\mu_{k}^{z}\left(\left\{e_{r_{k}}\right\}\right)=\left[\mu_{0}^{z}\left(\left\{e_{1}\right\}\right), \ldots, \mu_{0}^{z}\left(\left\{e_{\ell_{s}}\right\}\right)\right]\left(\Pi_{z}\right)^{k} e_{r_{k}}
$$

or, equivalently,

$$
\left[\mu_{k}^{z}\left(\left\{e_{1}\right\}\right), \ldots, \mu_{k}^{z}\left(\left\{e_{\ell_{s}}\right\}\right)\right]=\left[\mu_{0}^{z}\left(\left\{e_{1}\right\}\right), \ldots, \mu_{0}^{z}\left(\left\{e_{\ell_{o}}\right\}\right)\right]\left(\Pi_{z}\right)^{k}
$$

This last expression is the Kolmogorov equation of a Markov chain in $\Sigma_{s}$ with initial distribution $\left[\mu_{0}^{z}\left(\left\{e_{1}\right\}\right), \ldots, \mu_{0}^{z}\left(\left\{e_{\ell_{s}}\right\}\right)\right]$ (that is, $\left.\mu_{0}^{z}\right)$ and transition probability ma$\operatorname{trix} \Pi_{z}=\left[p_{r s}^{z}\right]$. Therefore, it follows from Kolmogorov's Existence Theorem that 
$\{z(k), k \geq 0\}$ is a Markov chain.

Note that in the context of this lemma, Remark IV.3.1 implies that $\Pi_{z}=$ $\sum_{i=0}^{\ell_{N}-1} S_{i, l}^{\mathrm{T}} p_{i}$. The lemma that follows shows that a similar derivation is possible when $\{N(k), k \geq 0\}$ is a Markov chain.

Lemma IV.4.2 Consider a canonical HJLS with state evolution equation (80). Let Assumption IV.3.1 hold and assume that for some $l \in \mathcal{I}_{\ell_{\nu}}, R_{l} \in \mathbb{R}^{n} \backslash \psi$ is a common invariant subspace. Let $\boldsymbol{x}(0)=\bar{x}_{l} \in R_{l}$. If $\{\boldsymbol{N}(k), k \geq 0\}$ is a Markov chain, then $\{(z(k), N(k)), k \geq 0\}$ is a Markov chain with initial distribution $\mu_{0}^{(z, N)}$ and transition probability matrix $\Pi_{(z, N)}=\left[p_{(r, i),(s, j)}^{(z, N)}\right]$ with

$$
p_{(r, i),(s, j)}^{(z, N)} \triangleq \operatorname{P}_{(r, i),(s, j)}\left(\bar{x}_{l}\right)=\mathbf{1}_{\left\{e_{s}\right\}}\left(S_{i, l} e_{r}\right) p_{i, j}^{N}
$$

Proof: Using a reasoning similar to the one in the proof of Lemma IV.4.1, it follows that every term of the form $\underset{\left(r_{k-1}, i_{k-1}\right),\left(r_{k}, i_{k}\right)}{\mathrm{P}}\left(A_{e_{r_{k-2}}} \cdots A_{e_{r_{0}}} \bar{x}\right)$ in (89) can be simplified as follows:

$$
\underset{\left(r_{k-1}, i_{k-1}\right),\left(r_{k}, i_{k}\right)}{\mathrm{P}}\left(A_{e_{r_{k-2}}} \cdots A_{e_{r_{0}}} \bar{x}\right)=p_{\left(r_{k-1}, i_{k-1}\right),\left(r_{k}, i_{k}\right)}^{(z, N)}
$$

Observe that $\mu_{k}^{(z, N)}\left(\left\{\left(e_{r_{k}}, i_{k}\right)\right\}\right)=\mu_{k}^{(y, N)}\left(\mathbb{R}^{n} \times\left\{\left(e_{r_{k}}, i_{k}\right)\right\}\right)$. Thus, after replacing the expression above in (89) and simplifying, it follows that

$$
\begin{gathered}
\mu_{k}^{(z, N)}\left(\left\{e_{r_{k}}, i_{k}\right\}\right)= \\
\sum_{r_{0}=1}^{\ell_{S}} \sum_{i_{0}=0}^{\ell_{N}-1} \cdots \sum_{r_{k-1}=1}^{\ell_{S}} \sum_{i_{k-1}=0}^{\ell_{N}-1} \mu_{0}^{(z, N)}\left(\left\{\left(e_{r_{0}}, i_{0}\right)\right\}\right) p_{\left(r_{0}, i_{0}\right),\left(r_{1}, i_{1}\right)}^{(z, N)} p_{\left(r_{1}, i_{1}\right),\left(r_{2}, i_{2}\right)}^{(z, N)} \cdots \\
p_{\left(r_{k-2}, i_{k-2}\right),\left(r_{k-1}, i_{k-1}\right)}^{(z, N)} p_{\left(r_{k-1}, i_{k-1}\right),\left(r_{k}, i_{k}\right)}^{(z, N)} .
\end{gathered}
$$


Finally, as in Lemma IV.4.1, it is possible to conclude that

$$
\begin{aligned}
& {\left[\mu_{k}^{(z, N)}\left(\left\{\left(e_{1}, 0\right)\right\}\right), \mu_{k}^{(z, N)}\left(\left\{\left(e_{1}, 1\right)\right\}\right), \ldots, \mu_{k}^{(z, N)}\left(\left\{\left(e_{\ell_{s}}, \ell_{N}-1\right)\right\}\right)\right]} \\
& \quad=\left[\mu_{0}^{(z, N)}\left(\left\{\left(e_{1}, 0\right)\right\}\right), \mu_{0}^{(z, N)}\left(\left\{\left(e_{1}, 1\right)\right\}\right), \ldots, \mu_{0}^{(z, N)}\left(\left\{\left(e_{\ell_{s}}, \ell_{N}-1\right)\right\}\right)\right]\left(\Pi_{(z, N)}\right)^{k}
\end{aligned}
$$

which in turn proves that $\{(z(k), N(k)), k \geq 0\}$ is a Markov chain with initial distribution $\mu_{0}^{(z, N)}$ and transition probability matrix $\Pi_{(z, N)}=\left[p_{(r, i),(s, j)}^{(z, N)}\right]$.

Remark IV.4.1 Observe that the effect of requiring that $\boldsymbol{x}(0)=\bar{x}_{l} \in R_{l}$, is to induce a restriction on the supervisor. That is, the supervisor's FSM is now given by $\mathrm{M}_{S p_{l}} \triangleq\left(\mathcal{I}_{\ell_{N}}, \Sigma_{S}, \mathcal{I}_{\ell_{O}}, \mathcal{S}_{l}, \varpi, \mu_{0}^{z}\right)$, where $\mathcal{S}_{l} \triangleq\left\{S_{\eta, \nu} \in \mathcal{S} \mid \eta \in \mathcal{I}_{\ell_{N}}, \nu=l\right\}$. Hence, under the conditions of Lemmas IV.4.1 and IV.4.2, the HJLS becomes a jump linear system driven by a FSM with a Markovian (or i.i.d.) input.

In light of Remark IV.4.1, it is now possible to state the main result of this subsection.

Theorem IV.4.1 Consider a canonical HJLS with state evolution equation (80). Let $\mathfrak{s}$ be the set of all its stochastic motions with initial condition $\boldsymbol{x}(0)=\bar{x}_{l} \in R_{l}, l \in \mathcal{I}_{\ell_{\nu}}$, $R_{l} \in \mathbb{R}^{n} \backslash \psi$. If $R_{l}$ is a common invariant subspace and Assumption IV.3.1 holds, the following statements are true.

(i) If $\{N(k), k \geq 0\}$ is an i.i.d. process, the dynamical system $\left\{\mathbb{Z}^{+}, Y, d, \mathfrak{s}\right\}$ is mean square stable whenever $\rho\left(\mathcal{A}_{\text {iid }}\right)<1$, where

$$
\mathcal{A}_{i i d}=\left(\Pi_{z}^{\mathrm{T}} \otimes I_{n^{2}}\right) \operatorname{diag}\left(A_{1} \otimes A_{1}, \ldots, A_{\ell_{S}} \otimes A_{\ell_{S}}\right)
$$

and $\Pi_{z}$ is defined as in Lemma IV.4.1. 
(ii) If $\{\boldsymbol{N}(k), k \geq 0\}$ is a Markov chain, the dynamical system $\left\{\mathbb{Z}^{+}, Y, d, \mathfrak{s}\right\}$ is mean square stable whenever $\rho\left(\mathcal{A}_{M C}\right)<1$, where

$$
\begin{aligned}
& \mathcal{A}_{M C}= \\
& \quad\left(\Pi_{(z, N)}^{\mathrm{T}} \otimes I_{n^{2}}\right) \operatorname{diag}\left(A_{(1,0)} \otimes A_{(1,0)}, A_{(1,1)} \otimes A_{(1,1)} \ldots, A_{\left(\ell_{s}, \ell_{N}-1\right)} \otimes A_{\left(\ell_{s}, \ell_{N}-1\right)}\right), \\
& \Pi_{(z, N)} \text { is defined as in Lemma IV.4.2, and } A_{(i, j)}=A_{i} \text { for every } i \in\left\{1, \ldots, \ell_{S}\right\} \\
& \text { and } j \in \mathcal{I}_{\ell_{N}} .
\end{aligned}
$$

Proof: To show (i), recall from Lemma IV.4.1 that $\{z(k), k \geq 0\}$ is a Markov chain. Therefore, the jump linear system in (80) is a Markov jump linear system and the spectral radius condition in (i) follows from (Costa \& Fragoso 1993). To show (ii), recall from Lemma IV.4.2 that $\{(z(k), N(k)), k \geq 0\}$ is a Markov chain. Thus, (ii) follows directly from Theorems 2.2 and 3.5 in (Patilkulkarni et al. 2004).

\section{IV.4.2 MS Stability Tests Based on Theorem III.5.2}

The stability tests developed in this subsection rely on Theorem III.5.2, which provides a sufficient MS stability test for a jump linear system of the form

$$
\boldsymbol{x}(k+1)=A_{\boldsymbol{\theta}(k)} \boldsymbol{x}(k),
$$

where $\{\boldsymbol{\theta}(k), k \geq 0\}$ represents any discrete-time stochastic process taking values from the set $\left\{1, \ldots, \ell_{O}\right\}$. The test consists on determining if $\rho\left(\mathcal{A}_{M_{\theta}}\right)<1$, where

$$
\mathcal{A}_{M_{\theta}} \triangleq\left(M_{\theta}^{\mathrm{T}} \otimes I_{n^{2}}\right) \operatorname{diag}\left(A_{1} \otimes A_{1}, \ldots, A_{\ell_{O}} \otimes A_{\ell_{O}}\right)
$$


and $M_{\theta}=\left[M_{i, j}\right]$ is an upper bound matrix. That is, the entries $M_{i, j}$ satisfy the equation

$$
\mathbf{E}\left\{\mathbf{1}_{\{\boldsymbol{\theta}(k+1)=j\}} \mid \mathscr{F}_{k}\right\} \mathbf{1}_{\{\boldsymbol{\theta}(k)=i\}} \leq M_{i, j} \mathbf{1}_{\{\boldsymbol{\theta}(k)=i\}},
$$

where

$$
\begin{aligned}
& \mathbf{E}\left\{\mathbf{1}_{\{\boldsymbol{\theta}(k+1)=j\}} \mid \mathscr{F}_{k}\right\} \mathbf{1}_{\{\boldsymbol{\theta}(k)=i\}}= \\
& \sum_{l_{0}=0}^{\ell_{O}-1} \cdots \sum_{l_{k-1}=0}^{\ell_{O}-1} \operatorname{Pr}\left\{\boldsymbol{\theta}(k+1)=j \mid \boldsymbol{\theta}(k)=i, \boldsymbol{\theta}(k-1)=l_{k-1}, \ldots, \boldsymbol{\theta}(0)=l_{0}\right\} \\
& \mathbf{1}_{\left\{\boldsymbol{\theta}(k)=i, \boldsymbol{\theta}(k-1)=l_{k-1}, \ldots, \boldsymbol{\theta}(0)=l_{0}\right\}}
\end{aligned}
$$

In Chapter III, it was shown that this theorem could be used to study HJLS's with performance map. Appropriate bounds, $M_{i, j}$, were computed assuming that $\{\boldsymbol{\theta}(k), k \geq 0\}$ represented the output sequences of the embedded FSM's. The goal in this subsection is to extend this analysis to included canonical HJLS's, and then to all HJLS's with embedded Moore-type FSM's. Recall that for a canonical HJLS, $\boldsymbol{\theta}(k)=\boldsymbol{z}(k)$. Thus, the expression above can be rewritten as follows:

$$
\begin{aligned}
& \mathbf{E}\left\{\mathbf{1}_{\left\{\boldsymbol{z}(k+1)=e_{s}\right\}} \mid \mathscr{F}_{k}\right\} \mathbf{1}_{\left\{\boldsymbol{z}(k)=e_{r}\right\}} \\
& \sum_{r_{0}=1}^{\ell_{S}} \cdots \sum_{r_{k-1}=1}^{\ell_{S}} \operatorname{Pr}\left\{\boldsymbol{z}(k+1)=e_{s} \mid \boldsymbol{z}(k)=e_{r}, \boldsymbol{z}(k-1)=e_{r_{k-1}}, \ldots, \boldsymbol{z}(0)=e_{r_{0}}\right\} \\
& \mathbf{1}_{\left\{\boldsymbol{z}(k)=e_{r}, \boldsymbol{z}(k-1)=e_{r_{k-1}}, \ldots, \boldsymbol{z}(0)=e_{r_{0}}\right\}} .
\end{aligned}
$$

Now, suppose that $\mu_{0}^{x}=\delta_{\bar{x}}$, that is, $\boldsymbol{x}(0)=\bar{x}$ for some fixed $\bar{x} \in \mathbb{R}^{n}$. Observe that if $\boldsymbol{z}(k-1)=e_{r_{k-1}}, \ldots, \boldsymbol{z}(0)=e_{r_{0}}$ for any fixed sequence of indexes $r_{0}, \ldots, r_{k-1}$, then $\boldsymbol{x}(k)=A_{r_{k-1}} \cdots A_{r_{0}} \bar{x}$. If, in addition, Assumption IV.3.1 holds then $\operatorname{Pr}\{\boldsymbol{z}(k+1)=$ 
$\left.e_{s} \mid z(k)=e_{r}, z(k-1)=e_{r_{k-1}}, \ldots, z(0)=e_{r_{0}}\right\}$ in (91) can be expressed as follows

$$
\begin{aligned}
\operatorname{Pr}\{\boldsymbol{z}(k+1) & \left.=e_{s} \mid \boldsymbol{z}(k)=e_{r}, \boldsymbol{z}(k-1)=e_{r_{k-1}}, \ldots, \boldsymbol{z}(0)=e_{r_{0}}\right\} \\
= & \frac{\operatorname{Pr}\left\{\boldsymbol{z}(k+1)=e_{s}, \boldsymbol{z}(k)=e_{r}, \boldsymbol{z}(k-1)=e_{r_{k-1}}, \ldots, \boldsymbol{z}(0)=e_{r_{0}}\right\}}{\operatorname{Pr}\left\{\boldsymbol{z}(k)=e_{r}, \boldsymbol{z}(k-1)=e_{r_{k-1}}, \ldots, \boldsymbol{z}(0)=e_{r_{0}}\right\}} \\
= & \frac{\operatorname{Pr}\left\{\boldsymbol{z}(k+1)=e_{s}, \boldsymbol{x}(k+1)=A_{r} A_{r_{k-1}} \cdots A_{r_{0}} \bar{x}, \ldots, \boldsymbol{z}(0)=e_{r_{0}}, \boldsymbol{x}(0)=\bar{x}\right\}}{\operatorname{Pr}\left\{\boldsymbol{z}(k)=e_{\boldsymbol{r}}, \boldsymbol{x}(k)=A_{r_{k-1}} \cdots A_{r_{0}} \bar{x}, \ldots, \boldsymbol{z}(0)=e_{r_{0}}, \boldsymbol{x}(0)=\bar{x}\right\}}
\end{aligned}
$$

or, equivalently,

$$
\begin{array}{r}
\operatorname{Pr}\left\{\boldsymbol{z}(k+1)=e_{s} \mid \boldsymbol{z}(k)=e_{r}, \boldsymbol{z}(k-1)=e_{r_{k-1}}, \ldots, \boldsymbol{z}(0)=e_{r_{0}}\right\}= \\
\operatorname{Pr}\left\{\boldsymbol{z}(k+1)=e_{s}, \boldsymbol{x}(k+1)=A_{r} A_{r_{k-1}} \cdots A_{r_{0}} \bar{x} \mid \boldsymbol{z}(k)=e_{r}, \boldsymbol{x}(k)=A_{r_{k-1}} \ldots\right. \\
\left.A_{r_{0}} \bar{x}, \ldots, \boldsymbol{z}(0)=e_{r_{0}}, \boldsymbol{x}(0)=\bar{x}\right\}
\end{array}
$$

Equation (92) is the basis of the proof of the following result.

Theorem IV.4.2 Consider the stochastic dynamical system $\left\{\mathbb{Z}^{+}, Y, d, \mathfrak{S}\right\}$ associated with a canonical HJLS with state evolution equation (80). Let Assumption IV.3.1 hold and assume that $\{\boldsymbol{N}(k), k \geq 0\}$ is an i.i.d. process. For every element $R_{l} \in \mathbb{R}^{n} \backslash \psi$, choose an arbitrary element $\alpha_{l} \in R_{l}$ and define $M_{z} \triangleq\left[M_{r, s}\right]$, where

$$
M_{r, s}=\max _{l \in \mathcal{I}_{\ell_{\nu}}}\left\{\underset{r, s}{\mathrm{P}}\left(\alpha_{l}\right)\right\}
$$

and $\underset{r, s}{\mathrm{P}}\left(\alpha_{l}\right)$ is defined as in $(77)$. Then, $\left\{\mathbb{Z}^{+}, Y, d, \mathfrak{S}\right\}$ is mean square stable if $\rho\left(\mathcal{A}_{M_{z}}\right)<$ 1 , where

$$
\mathcal{A}_{M_{z}} \triangleq\left(M_{z}^{\mathrm{T}} \otimes I_{n^{2}}\right) \operatorname{diag}\left(A_{1} \otimes A_{1}, \ldots, A_{\ell_{S}} \otimes A_{\ell_{S}}\right)
$$

Proof: Note that the HJLS defined above satisfies the conditions of Theorem III.5.2. It is only needed to show that $M_{z}$ is an upper bound matrix in the sense of Definition III.5.1. To this end, recall from Lemma IV.2.1 that when $\{N(k), k \geq 0\}$ is an 
i.i.d. process, $\{\boldsymbol{y}(k), k \geq 0\}$ is a Markov chain in $Y$ with transition kernel given by $\mathrm{P}_{y}\left(\left[\begin{array}{c}x \\ e_{r}\end{array}\right], B \times C\right)=\sum_{l=0}^{\ell_{N}-1} \mathbf{1}_{B}\left(A_{e_{r}} x\right) \mathbf{1}_{C}\left(S_{l, \psi(x)} e_{r}\right) p_{l}$. Hence, it follows directly from (92) that

$$
\begin{aligned}
\operatorname{Pr} & \left\{\boldsymbol{z}(k+1)=e_{s} \mid \boldsymbol{z}(k)=e_{r}, \boldsymbol{z}(k-1)=e_{r_{k-1}}, \ldots, \boldsymbol{z}(0)=e_{r_{0}}\right\} \\
& =\operatorname{Pr}\left\{\boldsymbol{z}(k+1)=e_{s}, \boldsymbol{x}(k+1)=A_{r} A_{r_{k-1}} \cdots A_{r_{0}} \bar{x} \mid \boldsymbol{z}(k)=e_{r}, \boldsymbol{x}(k)=A_{r_{k-1}} \cdots A_{r_{0}} \bar{x}\right\} \\
& =\mathrm{P}_{y}\left(\left[{ }_{r_{r_{k-1}} \cdots A_{r_{0}} \bar{x}}^{e_{r}}\right],\left\{A_{r} A_{r_{k-1}} \cdots A_{r_{0}} \bar{x}\right\} \times\left\{e_{s}\right\}\right) \\
& =\sum_{l=0}^{\ell_{N}-1} \mathbf{1}_{\left\{A_{r} A_{r_{k-1}} \cdots A_{r_{0}} \bar{x}\right\}}\left(A_{r} A_{r_{k-1}} \cdots A_{r_{0}} \bar{x}\right) \mathbf{1}_{\left\{e_{s}\right\}}\left(S_{l, \psi\left(A_{r_{k-1}} \cdots A_{r_{0}} \bar{x}\right)} e_{r}\right) p_{l} \\
& =\sum_{l=0}^{\ell_{N}-1} \mathbf{1}_{\left\{e_{s}\right\}}\left(S_{l, \psi\left(A_{r_{k-1}} \cdots A_{r_{0}} \bar{x}\right)} e_{r}\right) p_{l} \\
& =\mathrm{P}_{r, s}\left(A_{r_{k-1}} \cdots A_{r_{0}} \bar{x}\right) .
\end{aligned}
$$

The last step above follows from (77). Note that $A_{r_{k-1}} \cdots A_{r_{0}} \bar{x} \in R_{l}$ for some $l \in \mathcal{I}_{\ell_{\nu}}$. Thus,

$$
\underset{r, s}{\mathrm{P}}\left(A_{r_{k-1}} \cdots A_{r_{0}} \bar{x}\right)=\underset{r, s}{\mathrm{P}}\left(\alpha_{l}\right) \leq \max _{l \in \mathcal{I}_{\ell_{\nu}}}\left\{\underset{r, s}{\mathrm{P}}\left(\alpha_{l}\right)\right\}=M_{r, s}
$$

Using this inequality in (91) yields

$$
\begin{aligned}
\mathbf{E}\left\{\mathbf{1}_{\left\{\boldsymbol{z}(k+1)=e_{s}\right\}} \mid \mathscr{F}_{k}\right\} \mathbf{1}_{\left\{z(k)=e_{r}\right\}} & \leq \sum_{r_{0}=1}^{\ell_{S}} \cdots \sum_{r_{k-1}=1}^{\ell_{S}} M_{r, s} \mathbf{1}_{\left\{z(k)=e_{r}, \boldsymbol{z}(k-1)=e_{r_{k-1}}, \ldots, \boldsymbol{z}(0)=e_{r_{0}}\right\}} \\
& \leq M_{r, s} \mathbf{1}_{\left\{z(k)=e_{r}\right\}} \sum_{r_{0}=1}^{\ell_{S}} \cdots \sum_{r_{k-1}=1}^{\ell_{S}} \mathbf{1}_{\left\{z(k-1)=e_{r_{k-1}}, \ldots, \boldsymbol{z}(0)=e_{r_{0}}\right\}} \\
& \leq M_{r, s} \mathbf{1}_{\left\{z(k)=e_{r}\right\}}
\end{aligned}
$$

This last expression shows that $M_{z}=\left[M_{r, s}\right]$ is an upper bound matrix in the sense of Definition III.5.1. Thus, Theorem III.5.2 implies that if $\rho\left(\mathcal{A}_{M_{z}}\right)<1$ then 
$\left\{\mathbb{Z}^{+}, Y, d, \mathfrak{S}\right\}$ is mean square stable, completing the proof.

A similar result can be derived when $\{N(k), k \geq 0\}$ is a Markov chain. This result, however, cannot be derived using the same technique that led to (92) directly. To understand this, recall from the discussion leading to (92) that

$$
\begin{aligned}
& \operatorname{Pr}\left\{\boldsymbol{z}(k+1)=e_{s}, \boldsymbol{z}(k)=e_{r}, \boldsymbol{z}(k-1)=e_{r_{k-1}}, \ldots, \boldsymbol{z}(0)=e_{r_{0}}\right\} \\
& \quad=\operatorname{Pr}\left\{\boldsymbol{z}(k+1)=e_{s}, \boldsymbol{x}(k+1)=A_{r} A_{r_{k-1}} \cdots A_{r_{0}} \bar{x}, \ldots, \boldsymbol{z}(0)=e_{r_{0}}, \boldsymbol{x}(0)=\bar{x}\right\}
\end{aligned}
$$

This expression was derived under Assumption IV.3.1 holds with $\mu_{0}^{x}=\delta_{\bar{x}}$ and for a fixed sequence of integers $r_{0}, \ldots, r_{k-1}$. The right hand side of this expression can be rewritten as

$$
\begin{gathered}
\operatorname{Pr}\left\{\boldsymbol{z}(k+1)=e_{s}, \boldsymbol{x}(k+1)=A_{r} A_{r_{k-1}} \cdots A_{r_{0}} \bar{x}, \ldots, \boldsymbol{z}(0)=e_{r_{0}}, \boldsymbol{x}(0)=\bar{x}\right\}= \\
\operatorname{Pr}\left\{\boldsymbol{z}(k+1)=e_{s}, \boldsymbol{x}(k+1)=A_{r} A_{r_{k-1}} \cdots A_{r_{0}} \bar{x}, N(k+1) \in \mathcal{I}_{\ell_{N}}, \ldots,\right. \\
\left.\boldsymbol{z}(0)=e_{r_{0}}, \boldsymbol{x}(0)=\bar{x}, N(0) \in \mathcal{I}_{\ell_{N}}\right\}
\end{gathered}
$$

or, equivalently,

$$
\begin{array}{r}
\operatorname{Pr}\left\{\boldsymbol{z}(k+1)=e_{s}, \boldsymbol{x}(k+1)=A_{r} A_{r_{k-1}} \cdots A_{r_{0}} \bar{x}, \ldots, \boldsymbol{z}(0)=e_{r_{0}}, \boldsymbol{x}(0)=\bar{x}\right\}= \\
\sum_{l_{0}=0}^{\ell_{N}-1} \cdots \sum_{l_{k+1}=0}^{\ell_{N}-1} \operatorname{Pr}\left\{\boldsymbol{z}(k+1)=e_{s}, \boldsymbol{x}(k+1)=A_{r} A_{r_{k-1}} \cdots A_{r_{0}} \bar{x}, \boldsymbol{N}(k+1)=l_{k+1}\right. \\
\left.\ldots, \boldsymbol{z}(0)=e_{r_{0}}, \boldsymbol{x}(0)=\bar{x}, \boldsymbol{N}(0)=l_{0}\right\}
\end{array}
$$

Now recall that the process $\{(\boldsymbol{y}(k), \boldsymbol{N}(k)), k \geq 0\}$ is a Markov chain. So after multiplying and dividing the equality above by $\operatorname{Pr}\left\{\boldsymbol{z}(k)=e_{r}, \boldsymbol{x}(k)=\right.$ $\left.A_{r_{k-1}} \cdots A_{r_{0}} \bar{x}, \boldsymbol{N}(k)=l_{k}, \ldots, \boldsymbol{z}(0)=e_{r_{0}}, \boldsymbol{x}(0)=\bar{x}, \boldsymbol{N}(0)=l_{0}\right\}$, using the definition of conditional probability, and using the Markov property of $\{(\boldsymbol{y}(k), \boldsymbol{N}(k)), k \geq 0\}$, 
it follows that

$$
\begin{gathered}
\operatorname{Pr}\left\{\boldsymbol{z}(k+1)=e_{s}, \boldsymbol{x}(k+1)=A_{r} A_{r_{k-1}} \cdots A_{r_{0}} \bar{x}, \ldots, \boldsymbol{z}(0)=e_{r_{0}}, \boldsymbol{x}(0)=\bar{x}\right\}= \\
\sum_{l_{0}=0}^{\ell_{N}-1} \cdots \sum_{l_{k+1}=0}^{\ell_{N}-1} \mathrm{P}_{(y, N)}\left(\left(\left[\begin{array}{c}
A_{r_{k-1}} \cdots A_{r_{r}} \bar{x} \\
\boldsymbol{e}_{r}
\end{array}\right], l_{k}\right),\left\{A_{r} A_{r_{k-1}} \cdots A_{r_{0}} \bar{x}\right\} \times\left\{e_{s}\right\} \times\left\{l_{k+1}\right\}\right) \\
\operatorname{Pr}\left\{\boldsymbol{z}(k)=e_{r}, \boldsymbol{x}(k)=A_{r_{k-1}} \cdots A_{r_{0}} \bar{x}, \boldsymbol{N}(k)=l_{k}, \ldots, \boldsymbol{z}(0)=e_{r_{0}}, \boldsymbol{x}(0)=\bar{x}, \boldsymbol{N}(0)=l_{0}\right\}
\end{gathered}
$$

where

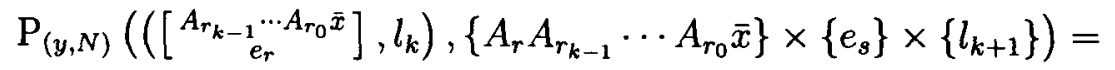

$$
\begin{aligned}
& \operatorname{Pr}\left\{\boldsymbol{z}(k+1)=e_{s}, \boldsymbol{x}(k+1)=A_{r} A_{r_{k-1}} \cdots A_{r_{0}} \bar{x}, \boldsymbol{N}(k+1)=l_{k+1} \mid\right. \\
& \left.\boldsymbol{z}(k)=e_{r}, \boldsymbol{x}(k)=A_{r_{k-1}} \cdots A_{r_{0}} \bar{x}, \boldsymbol{N}(k)=l_{k}\right\}
\end{aligned}
$$

and $\mathrm{P}_{(y, N)}$ is the Markov kernel associated with $\{(\boldsymbol{y}(k), \boldsymbol{N}(k)), k \geq 0\}$ defined in (84), with $B=\left\{A_{r} A_{r_{k-1}} \cdots A_{r_{0}} \bar{x}\right\}, C=\left\{e_{s}\right\}$, and $D=\left\{l_{k+1}\right\}$. Note from (75) and (84) that

$$
\begin{aligned}
& \mathrm{P}_{(y, N)}\left(\left(\left[\begin{array}{c}
A_{r_{k-1} \cdots A_{r_{0}} \bar{x}} \\
e_{r}
\end{array}\right], l_{k}\right),\left\{A_{r} A_{r_{k-1}} \cdots A_{r_{0}} \bar{x}\right\} \times\left\{e_{s}\right\} \times\left\{l_{k+1}\right\}\right) \\
& =\underset{\left(r, l_{k}\right),\left(s, l_{k+1}\right)}{\mathrm{P}}\left(A_{r_{k-1}} \cdots A_{r_{0}} \bar{x}\right)
\end{aligned}
$$

which in turn implies that

$$
\begin{array}{r}
\operatorname{Pr}\left\{\boldsymbol{z}(k+1)=e_{s}, \boldsymbol{x}(k+1)=A_{r} A_{r_{k-1}} \cdots A_{r_{0}} \bar{x}, \ldots, \boldsymbol{z}(0)=e_{r_{0}}, \boldsymbol{x}(0)=\bar{x}\right\}= \\
\sum_{l_{0}=0}^{\ell_{N}-1} \cdots \sum_{l_{k+1}=0}^{\ell_{N}-1} \underset{\left(r, l_{k}\right),\left(s, l_{k+1}\right)}{\mathrm{P}}\left(A_{r_{k-1}} \cdots A_{r_{0}} \bar{x}\right) \operatorname{Pr}\left\{\boldsymbol{z}(k)=e_{r}, \boldsymbol{x}(k)=A_{r_{k-1}} \cdots A_{r_{0}} \bar{x}\right. \\
\left.\boldsymbol{N}(k)=l_{k}, \ldots, \boldsymbol{z}(0)=e_{r_{0}}, \boldsymbol{x}(0)=\bar{x}, \boldsymbol{N}(0)=l_{0}\right\} .
\end{array}
$$

Note that

$$
\sum_{l_{k+1}=0}^{\ell_{N}-1} \underset{\left(r, l_{k}\right),\left(s, l_{k+1}\right)}{\mathrm{P}}\left(A_{r_{k-1}} \cdots A_{r_{0}} \bar{x}\right)=\underset{\left(r, l_{k}\right),\left(s, I_{\ell_{N}}\right)}{\mathrm{P}}\left(A_{r_{k-1}} \cdots A_{r_{0}} \bar{x}\right)
$$


This latter expression is either equal to 1 , when $e_{s}=S_{l_{k}, \psi\left(A_{r_{k-1}} \cdots A_{r_{0}} \bar{x}\right)} e_{r}$, or equal to 0 otherwise. Thus,

$$
\begin{array}{r}
\operatorname{Pr}\left\{\boldsymbol{z}(k+1)=e_{\boldsymbol{s}}, \boldsymbol{x}(k+1)=A_{r} A_{r_{k-1}} \cdots A_{r_{0}} \bar{x}, \ldots, \boldsymbol{z}(0)=e_{r_{0}}, \boldsymbol{x}(0)=\bar{x}\right\} \leq \\
\sum_{l_{0}=0}^{\ell_{N}-1} \cdots \sum_{l_{k}=0}^{\ell_{N}-1} \operatorname{Pr}\left\{\boldsymbol{z}(k)=e_{r}, \boldsymbol{x}(k)=A_{r_{k-1}} \cdots A_{r_{0}} \bar{x}, \boldsymbol{N}(k)=l_{k}, \ldots, \boldsymbol{z}(0)=e_{r_{0}},\right. \\
\left.\boldsymbol{x}(0)=\bar{x}, \boldsymbol{N}(0)=l_{0}\right\},
\end{array}
$$

or, equivalently,

$$
\begin{aligned}
\operatorname{Pr}\left\{\boldsymbol{z}(k+1)=e_{s}, \boldsymbol{x}(k+1)\right. & \left.=A_{r} A_{r_{k-1}} \cdots A_{r_{0}} \bar{x}, \ldots, \boldsymbol{z}(0)=e_{r_{0}}, \boldsymbol{x}(0)=\bar{x}\right\} \leq \\
\operatorname{Pr}\{\boldsymbol{z}(k) & \left.=e_{r}, \boldsymbol{x}(k)=A_{r_{k-1}} \cdots A_{r_{0}} \bar{x}, \ldots, \boldsymbol{z}(0)=e_{r_{0}}, \boldsymbol{x}(0)=\bar{x}\right\}
\end{aligned}
$$

This, in turn, implies that the procedure described above can, at best, only upperbound the conditional probabilities in (92) with 1 . That is, the best bounds produced by this procedure are $M_{r, s}=1$. This is equivalent to the condition for stability under arbitrary switching of Theorem III.3.2, which obviously conservative.

This analysis can be significantly improved using the concept of A-equivalency. This concept was introduced by Patilkulkarni et al. (2004) in the context of Markov jump linear systems and can be extended to HJLS's. Briefly (see Appendix C for a full discussion of this concept), two jump linear systems

$$
\boldsymbol{x}(k+1)=A_{\boldsymbol{\theta}(k)} \boldsymbol{x}(k) \quad \text { and } \quad \tilde{\boldsymbol{x}}(k+1)=\tilde{A}_{\boldsymbol{\varphi}(k)} \tilde{\boldsymbol{x}}(k),
$$

are said to be A-equivalent if, for every $k \geq 0, A_{\boldsymbol{\theta}(k)}=\tilde{A}_{\boldsymbol{\varphi}(k)}$. Hence, two HJLS's are said to be A-equivalent if their jump linear closed-loop systems are A-equivalent. 
Consider the particular HJLS's

$$
\begin{aligned}
\boldsymbol{x}(k+1) & =A_{\boldsymbol{z}(k)} \boldsymbol{x}(k) \\
\boldsymbol{z}(k+1) & =S_{\boldsymbol{N}(k), \psi(\boldsymbol{x}(k))} \boldsymbol{z}(k) \\
\psi(x) & =\sum_{i=0}^{\ell_{\nu}-1} i \mathbf{1}_{\left\{x \in R_{i}\right\}}, \quad \bigcup_{i \in \mathcal{I}_{\ell_{\nu}}} R_{i}=\mathbb{R}^{n}
\end{aligned}
$$

and

$$
\begin{aligned}
\tilde{\boldsymbol{x}}(k+1) & =\tilde{A}_{\tilde{\boldsymbol{z}}(k), \boldsymbol{N}(k)} \tilde{\boldsymbol{x}}(k) \\
\tilde{\boldsymbol{z}}(k+1) & =S_{\boldsymbol{N}(k), \psi(\tilde{\boldsymbol{x}}(k))} \tilde{\boldsymbol{z}}(k) \\
\psi(x) & =\sum_{i=0}^{\ell_{\nu}-1} i \mathbf{1}_{\left\{x \in R_{i}\right\}} . \quad \bigcup_{i \in \mathcal{I}_{\ell_{\nu}}} R_{i}=\mathbb{R}^{n}
\end{aligned}
$$

As explained in Appendix C, these HJLS's are A-equivalent when they have the same $A / S$ maps, are initialized with the same initial state $\left[\begin{array}{ll}x_{0} & z_{0}\end{array}\right]$, and $A_{\boldsymbol{z}(k)}=$ $A_{\tilde{\boldsymbol{z}}(k), N(k)}=A_{r}$, whenever $\tilde{\boldsymbol{z}}(k)=\boldsymbol{z}(k)=e_{r}$. Furthermore, it is established in Corollary C.2.1 that if Assumption IV.3.1 also holds, then the MS stability of (94) is equivalent to the MS stability of (93). These ideas are the basis for the next two theorems. They constitute the main contribution of this dissertation.

Theorem IV.4.3 Consider the stochastic dynamical system $\left\{\mathbb{Z}^{+}, Y, d, \mathfrak{S}\right\}$ associated with the HJLS in (94). Assume that $\{N(k), k \geq 0\}$ is a Markov chain. Let Assumption IV.3.1 hold, ${ }^{3}$ and, for every set $R_{l} \in \mathbb{R}^{n} \backslash \psi$ choose an arbitrary element $\alpha_{l} \in R_{l}$. Finally, let $M_{(z, N)} \triangleq\left[M_{(r, i),(s, j)}\right]$, where

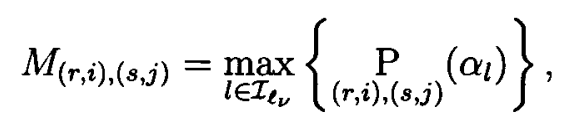

\footnotetext{
${ }^{3}$ In this context, Assumption IV.3.1 requires $\tilde{y}(0)$ to be independent of $\{N(k), k \geq 0\}$.
} 
and $\underset{(r, i),(s, j)}{\mathrm{P}}\left(\alpha_{l}\right)$ is defined as in $(75)$. If $\rho\left(\mathcal{A}_{M_{(z, N)}}\right)<1$, where

$$
\mathcal{A}_{M_{(z, N)}} \triangleq\left(M_{(z, N)}^{\mathrm{T}} \otimes I_{n^{2}}\right) \operatorname{diag}\left(A_{1,0} \otimes A_{1,0}, A_{1,1} \otimes A_{1,1}, \ldots, A_{\ell_{S}, \ell_{N}-1} \otimes A_{\ell_{S}, \ell_{N}-1}\right),
$$

then the HJLS in (94) is MS stable.

Proof: Let $\boldsymbol{\theta}(k)=(\tilde{\boldsymbol{z}}(k), \boldsymbol{N}(k))$ and rewrite the jump linear system in (94) as

$$
\tilde{\boldsymbol{x}}(k+1)=A_{\boldsymbol{\theta}(k)} \tilde{\boldsymbol{x}}(k),
$$

where $\boldsymbol{\theta}(k)$ takes values from $\Sigma_{S} \times \mathcal{I}_{\ell_{N}}$. As in Theorem IV.4.2, consider the conditional expectation

$$
\begin{aligned}
& \mathbf{E}\left\{\mathbf{1}_{\left\{\boldsymbol{\theta}(k+1)=\left(e_{s}, j\right)\right\}} \mid \mathscr{F}_{k}\right\} \mathbf{1}_{\left\{\boldsymbol{\theta}(k)=\left(e_{r}, i\right)\right\}}= \\
& \sum_{r_{0}=1}^{\ell_{S}} \sum_{i_{0}=0}^{\ell_{N}-1} \cdots \sum_{r_{k-1}=1}^{\ell_{S}} \sum_{i_{k-1}=0}^{\ell_{N}-1} \operatorname{Pr}\left\{\boldsymbol{\theta}(k+1)=\left(e_{s}, j\right) \mid \boldsymbol{\theta}(k)=\left(e_{r}, i\right), \boldsymbol{\theta}(k-1)=\left(e_{r_{k-1}}, i_{k-1}\right),\right. \\
& \left.\ldots, \boldsymbol{\theta}(0)=\left(e_{r_{0}}, i_{0}\right)\right\} \mathbf{1}_{\left\{\boldsymbol{\theta}(k)=(r, i), \boldsymbol{\theta}(k-1)=\left(e_{r_{k-1}}, i_{k-1}\right), \ldots, \boldsymbol{\theta}(0)=\left(e_{r_{0}}, i_{0}\right)\right\}} .
\end{aligned}
$$

Fix any initial condition $\left[\begin{array}{ll}\tilde{x}_{0}^{\mathrm{T}} & \tilde{z}_{0}^{\mathrm{T}}\end{array}\right]^{\mathrm{T}}$. A procedure similar to the one leading to (92) yields

$$
\begin{aligned}
& \operatorname{Pr}\left\{\boldsymbol{\theta}(k+1)=\left(e_{s}, j\right) \mid \boldsymbol{\theta}(k)=\left(e_{r}, i\right), \boldsymbol{\theta}(k-1)=\left(e_{r_{k-1}}, i_{k-1}\right), \ldots, \boldsymbol{\theta}(0)=\left(e_{r_{0}}, i_{0}\right)\right\} \\
& =\operatorname{Pr}\left\{\boldsymbol{\theta}(k+1)=\left(e_{s}, j\right), \tilde{\boldsymbol{x}}(k+1)=A_{e_{r}, i} \cdots A_{e_{r_{0}}, i_{0}} \bar{x} \mid \boldsymbol{\theta}(k)=\left(e_{r}, i\right),\right. \\
& \left.\tilde{\boldsymbol{x}}(k)=A_{e_{r_{k-1}}, i_{k-1}} \cdots A_{e_{r_{0}}, i_{0}} \bar{x}, \ldots, \boldsymbol{\theta}(0)=\left(e_{r_{0}}, i_{0}\right), \tilde{\boldsymbol{x}}(0)=\bar{x}\right\} .
\end{aligned}
$$

Note that this conclusion is valid under Assumption IV.3.1, since both $\tilde{\boldsymbol{x}}(k)$ and $\tilde{\boldsymbol{z}}(k)$ are functions for $N(k-1), \ldots, N(0)$, which in turn are independent of $\left[\tilde{x}_{0}^{\mathrm{T}} \tilde{z}_{0}^{\mathrm{T}}\right]^{\mathrm{T}}$. Now, let $\tilde{\boldsymbol{y}}(k)=\left[\tilde{\boldsymbol{x}}(k)^{\mathrm{T}} \tilde{\boldsymbol{z}}(k)^{\mathrm{T}}\right]^{\mathrm{T}}$, and observe that $(\tilde{\boldsymbol{y}}(k), \boldsymbol{N}(k))=(\tilde{\boldsymbol{x}}(k), \boldsymbol{\theta}(k))$, for every $k \geq 0$. Next, observe that

$$
\tilde{\boldsymbol{y}}(k+1)=\tilde{F}(\tilde{\boldsymbol{y}}(k), \boldsymbol{N}(k)),
$$


where $\tilde{F}$ is a measurable function given by

$$
\tilde{F}(x, z, l) \triangleq\left[\begin{array}{l}
\sum_{r=1}^{\ell_{S}} \sum_{i=0}^{\ell_{N}-1} A_{r, i} x \mathbf{1}_{\left\{z=e_{r}\right\}} \mathbf{1}_{\{l=i\}} \\
\sum_{i=0}^{\ell_{N-1}} \sum_{j=0}^{\ell_{\nu}-1} S_{i, j} z \mathbf{1}_{\left\{x \in R_{j}\right\}} \mathbf{1}_{\{l=i\}}
\end{array}\right]
$$

for every $x \in \mathbb{R}^{n}, z \in \Sigma_{S}$, and $l \in \mathcal{I}_{\ell_{N}}$. Hence, Theorem IV.2.1 implies that $\{(\tilde{\boldsymbol{y}}(k), \boldsymbol{N}(k)), k \geq 0\}$ and $\{(\tilde{\boldsymbol{x}}(k), \boldsymbol{\theta}(k)), k \geq 0\}$ are Markov chains in $Y \times \mathcal{I}_{\ell_{N}}$.

Consequently, (96) can be reduced as follows:

$$
\begin{array}{r}
\operatorname{Pr}\left\{\boldsymbol{\theta}(k+1)=\left(e_{s}, j\right) \mid \boldsymbol{\theta}(k)=\left(e_{r}, i\right), \boldsymbol{\theta}(k-1)=\left(e_{r_{k-1}}, i_{k-1}\right), \ldots, \boldsymbol{\theta}(0)=\left(e_{r_{0}}, i_{0}\right)\right\} \\
=\operatorname{Pr}\left\{\boldsymbol{\theta}(k+1)=\left(e_{s}, j\right), \tilde{\boldsymbol{x}}(k+1)=A_{e_{r}, i} \cdots A_{e_{r_{0}}, i_{0}} \bar{x} \mid \boldsymbol{\theta}(k)=\left(e_{r}, i\right)\right. \\
\left.\tilde{\boldsymbol{x}}(k)=A_{e_{r_{k-1}}, i_{k-1}} \cdots A_{e_{r_{0}}, i_{0}} \bar{x}\right\}
\end{array}
$$

Next, note that the Markov kernel of $\{(\tilde{\boldsymbol{y}}(k), \boldsymbol{N}(k)), k \geq 0\}$ is given by

$$
\mathrm{P}_{(\tilde{y}, N)}\left(\left(\left[\begin{array}{l}
x \\
e_{r}
\end{array}\right], i\right), B \times C \times D\right)=\sum_{j=0}^{\ell_{N}-1} \mathbf{1}_{B}\left(A_{e_{r}, i} e_{r}\right) \mathbf{1}_{C}\left(S_{i, \psi(x)} e_{r}\right) \mathbf{1}_{D}(j) p_{i, j}^{N},
$$

so the conditional probability above can be reduced to

$$
\begin{aligned}
& \operatorname{Pr}\left\{\boldsymbol{\theta}(k+1)=\left(e_{s}, j\right) \mid \boldsymbol{\theta}(k)=\left(e_{r}, i\right), \boldsymbol{\theta}(k-1)=\left(e_{r_{k-1}}, i_{k-1}\right), \ldots, \boldsymbol{\theta}(0)=\left(e_{r_{0}}, i_{0}\right)\right\} \\
& =\mathrm{P}_{(\bar{y}, N)}\left(\left(\left[\begin{array}{c}
A_{e_{r_{k-1}}, i_{k-1}} \cdots A_{e_{r_{0}}, i_{0}} \bar{x} \\
e_{r}
\end{array}\right], i\right),\left\{A_{e_{r}, i} A_{e_{r_{k-1}}, i_{k-1}} \cdots A_{e_{r_{0}}, i_{0}} \bar{x}\right\} \times\left\{e_{r_{s}}\right\} \times\{j\}\right) \\
& =\mathbf{1}_{\left\{e_{\left.r_{s}\right\}}\right\}}\left(S_{i, \psi\left(A_{e_{r_{k-1}}, i_{k-1}} \cdots A_{e_{r_{0}}, i_{0}} \bar{x}\right)} e_{r}\right) p_{i, j}^{N} \\
& =\underset{(r, i),(s, j)}{\mathrm{P}}\left(A_{e_{r_{k-1}}, i_{k-1}} \cdots A_{e_{r_{0}, i_{0}}} \bar{x}\right) .
\end{aligned}
$$

Now, $A_{e_{r_{k-1}}, i_{k-1}} \cdots A_{e_{r_{0}}, i_{0}} \bar{x} \in R_{l}$ for some set $R_{l}$. Thus,

$$
\underset{(r, i),(s, j)}{\mathrm{P}}\left(A_{e_{r_{k-1}}, i_{k-1}} \cdots A_{e_{r_{0}}, i_{0}} \bar{x}\right)=\operatorname{P}_{(r, i),(s, j)}^{\mathrm{P}}\left(\alpha_{l}\right) \leq \max _{l \in \mathcal{I}_{\ell_{\nu}}}\left\{\underset{(r, i),(s, j)}{\mathrm{P}}\left(\alpha_{l}\right)\right\}=M_{(r, i),(s, j)},
$$

and therefore

$$
\begin{array}{r}
\operatorname{Pr}\left\{\boldsymbol{\theta}(k+1)=\left(e_{s}, j\right) \mid \boldsymbol{\theta}(k)=\left(e_{r}, i\right), \boldsymbol{\theta}(k-1)=\left(e_{r_{k-1}}, i_{k-1}\right), \ldots, \boldsymbol{\theta}(0)\right. \\
\left.=\left(e_{r_{0}}, i_{0}\right)\right\} \\
\leq M_{(r, i),(s, j)} .
\end{array}
$$


This in turn implies that

$$
\mathbf{E}\left\{\mathbf{1}_{\left\{\boldsymbol{\theta}(k+1)=\left(e_{s}, j\right)\right\}} \mid \mathscr{F}_{k}\right\} \mathbf{1}_{\left\{\boldsymbol{\theta}(k)=\left(e_{r}, i\right)\right\}} \leq M_{(r, i),(s, j)} \mathbf{1}_{\left\{\boldsymbol{\theta}(k)=\left(e_{r}, i\right)\right\}}
$$

Note that this conclusion is independent of $\bar{x}$. Thus, $M_{(z, N)}$ is an upper bound matrix in the sense of Definition III.5.1, and Theorem III.5.2 implies that if $\rho\left(\mathcal{A}_{M_{(z, N)}}\right)<1$ then the HJLS is MS stable, completing the proof.

The last result of this chapter follows.

Theorem IV.4.4 Consider the stochastic dynamical system $\left\{\mathbb{Z}^{+}, Y, d, \mathfrak{S}\right\}$ associated with the canonical HJLS in (93). Assume that $\{N(k), k \geq 0\}$ is a Markov chain and that Assumption IV.3.1 holds. Let $M_{(z, N)}$ and $\mathcal{A}_{M_{(z, N)}}$ be defined as in Theorem IV.4.3. If $\rho\left(\mathcal{A}_{M_{(z, N)}}\right)<1$ then the canonical HJLS in (93) is $M S$ stable.

Proof: Consider any stochastic motion in $\mathfrak{s}$ with initial condition $\left[\begin{array}{ll}x_{0}^{\mathrm{T}} & z_{0}^{\mathrm{T}}\end{array}\right]^{\mathrm{T}}$. For this initial condition, Theorem IV.4.3 shows that if $\rho\left(\mathcal{A}_{M_{(z, N)}}\right)<1$ then $\mathrm{E}\left\{\|\tilde{\boldsymbol{x}}(k)\|^{2}\right\} \rightarrow 0$ as $k \rightarrow \infty$. But (93) and (94) are A-equivalent. Thus, it follows from Theorem C.2.1 that $\mathbf{E}\left\{\|\boldsymbol{x}(k)\|^{2}\right\} \rightarrow 0$ as $k \rightarrow \infty$. Since this is true for every stochastic motion in $\mathbf{5}$, then the HJLS in (93) is mean square stable.

Remark IV.4.2 The constants $M_{r, s}$ and $M_{(r, i),(s, j)}$ in Theorems IV.4.2 and IV.4.3 must be computed in order to apply the stability criteria. To do so, select constants $\alpha_{l} \in R_{l}$ with $l \in \ell_{\nu}$ and $R_{l} \in \mathbb{R}^{n} \backslash \psi$. Next, compute the sets $\left\{\underset{r, s}{\mathrm{P}}\left(\alpha_{0}\right), \ldots, \underset{r, s}{\mathrm{P}}\left(\alpha_{\ell_{\nu}-1}\right)\right\}$ and $\left\{\underset{(r, i),(s, j)}{\mathrm{P}}\left(\alpha_{0}\right), \ldots, \underset{(r, i),(s, j)}{\mathrm{P}}\left(\alpha_{\ell_{\nu}-1}\right)\right\}$. Finally, set $M_{r, s}=\max \left\{\underset{r, s}{\mathrm{P}}\left(\alpha_{0}\right), \ldots, \underset{r, s}{\mathrm{P}}\left(\alpha_{\ell_{\nu}-1}\right)\right\}$ and $M_{(r, i),(s, j)}=\max \left\{\underset{(r, i),(s, j)}{\mathrm{P}}\left(\alpha_{0}\right), \ldots, \underset{(r, i),(s, j)}{\mathrm{P}}\left(\alpha_{\ell_{\nu}-1}\right)\right\}$. 
TABLE IV: Summary of Main Results in Chapter IV.

\begin{tabular}{|l|c|l|}
\hline Technique & Theorem & \multicolumn{1}{|c|}{ Summary } \\
\hline $\begin{array}{l}\sigma \text {-algebra recombi- } \\
\text { nation }\end{array}$ & IV.2.1 & $\begin{array}{l}\text { Shows the Markov property for the solution of } \\
\text { non-linear difference equations with Markov in- } \\
\text { puts }\end{array}$ \\
\hline Invariant Subspaces & IV.4.1 & $\begin{array}{l}\text { Testable sufficient MSS condition for canonical } \\
\text { HJLS's }\end{array}$ \\
\hline $\begin{array}{l}\text { Apply Theorem } \\
\text { III.5.2 }\end{array}$ & IV.4.2 & $\begin{array}{l}\text { Testable sufficient MSS condition for canonical } \\
\text { HJLS's with i.i.d. inputs }\end{array}$ \\
\cline { 2 - 3 } & IV.4.4 & $\begin{array}{l}\text { Testable sufficient MSS condition for canonical } \\
\text { HJLS's with Markov inputs }\end{array}$ \\
\hline
\end{tabular}

The next chapter illustrates the modeling capabilities of the HJLS formalism and the usefulness of Theorems IV.4.1, IV.4.2 and IV.4.3 in accessing stability properties. The application is an AFTI-F16 aircraft control system deployed on a fault tolerant computer.

\section{IV.5 CHAPTER SUMMARY AND CONCLUSIONS}

This chapter has established that the state process of a HJLS constitutes a Markov chain in a metric space. Specific Markov kernels for the processes $\{(\boldsymbol{y}(k), \boldsymbol{N}(k)), k \geq$ $0\}$ and $\{\boldsymbol{y}(k), k \geq 0\}$ were derived assuming that $\{\boldsymbol{N}(k), k \geq 0\}$ is, respectively, a Markov chain or an i.i.d. process. The analysis of the evolution of the probability measures of these processes, $\mu_{k}^{(y, N)}$ and $\mu_{k}^{y}$, led to two testable sufficient MS stability conditions, Theorem IV.4.1. The analysis of the kernels associated with these processes, $P_{(y, N)}$ and $P_{y}$, led to two testable sufficient MS stability conditions for canonical 
HJLS's, Theorems IV.4.2 and IV.4.4, which are two of the main contributions of this dissertation. The latter was developed using the concept of A-equivalency, which is further developed in Appendix C. The main results of this chapter are summarized in Table IV. 


\section{CHAPTER V}

\section{SIMULATION EXAMPLE}

\section{V.1 INTRODUCTION}

This chapter aims to accomplish two goals. The first goal is to demonstrate that HJLS's constitute a valuable modeling tool for digital control systems deployed on computers equipped with advanced fault-tolerant mechanisms. The second goal is to illustrate the usefulness and limitations of Theorems IV.4.1, IV.4.2 and IV.4.3 in accessing stability characteristics. The first goal is attained by introducing a HJLS model for a specific Advanced Recovery Mechanism (ARM). This ARM combines the best features of two well-known fault recovery mechanisms, rollback recovery and coldrestart, and makes use of the data, models, and concepts described in (Tejada et al. 2007). The second goal is attained by analyzing the MS stability of the aforementioned HJLS using the tools introduced in Chapter IV (Tejada et al. 2007). These conclusions of this analysis are then verified via Monte Carlo simulations.

This chapter is organized as follows. Section V.2 motivates the need for fault recovery mechanisms and summarizes the basic features of rollback and cold-restart recovery. It also provides a brief summary of the dynamical models proposed in (Tejada et al. 2007) to study the effect of these recovery mechanisms on digital control systems. Section V.3 motivates and introduces the particular ARM under consideration. This section also provides the HJLS model for the ARM. Finally, the stability analysis of this HJLS is performed in Section V.4 using the stability tests of Chapter IV. Monte Carlo simulations are used to verify the theoretical predictions. 


\section{V.2 ON FAULT RECOVERY MECHANISMS AND THEIR DYNAMICAL MODELS}

\section{V.2.1 Motivation and Background}

The fault-tolerant community usually classifies the faults in a computer platform by either their impact (local or global), their duration (transient or permanent) and their origin (internal or externally-induced) (see Johnson 1989). Transient localized faults, regardless of their origin, are handled with standard fault-tolerant provisions, such as triple modular redundancy or error correcting codes, which are very effective in removing the effects induced by the faults, while operating unobtrusively. These provisions, however, are not effective against global (or pervasive) faults, which are able to affect several locations of the computer platform at once. These faults, called Common Mode Faults (CMF's), are usually handled with special fault recovery mechanisms that remove the faults by executing systematic recovery procedures. These procedures require the host computer to temporarily stop while the corrections are made. Consequently, the activity of an fault recovery mechanism impacts the overall behavior of the computer platform and its users. In particular, if the computer is executing a digital control law, it is possible for the closed-loop system to become unstable if the recovery mechanism is activated too frequently. Understanding this phenomena is of particular importance for life-critical applications such as commercial aircraft control systems or nuclear power plant controllers, which are subject to CMF's induced by, for example, lightning, radar beams, or nuclear electromagnetic pulses (cf. Tejada et al. 2007, Gray et al. 2000).

Section V.3 presents a novel ARM that combines features of both rollback and 
cold-restart recovery. These classical recovery mechanisms are described next based on the work of (Tejada et al. 2007).

\section{Rollback Recovery}

This is an information redundancy technique consisting of three steps: checkpointing, fault detection, and reload-and-retry. Checkpointing is a process that periodically stores a copy of the most relevant information from the computer platform's memory and registers into special protected memory. From a digital control system perspective, the relevant information corresponds to the value of the controller's state vector.

Fault detection is a continuous process that identifies the presence of a computer faults and triggers the reload-and-retry mechanism when necessary. The specific method used to detect the faults and the speed and coverage of the detection process varies depending on the particular hardware implementation. However, from a digital control system perspective, it can be assumed that the reload-and-retry mechanism is triggered immediately after the faults appear in the computer platform. That is, there is no delay between the appearance of a fault and its detection.

Reload-and-retry is the process that eliminates the effect of the faults in the computer platform. After being triggered, this process stops the checkpointing process, if active, freezes the computer platform's output ports and ceases all computing activities. Next, the information in the memory and registers of the computer platform is replaced by the information stored in the last completed checkpointing process. Finally, the output ports are freed and the computing activity is restarted. From a digital control system perspective, this process amounts to freezing the controller's output signals, reloading the controller's state vector with the latest error-free value, 
and then resuming normal operation.

\section{Cold-Restart Recovery}

This recovery technique is similar to rollback but simpler. Unlike rollback, cold-restart does not make use of the checkpointing operation, only the fault detection and reloadand-retry processes described above. From a digital control system perspective, the cold-restart process consists of freezing the controller's output signals, setting the controller's state vector to zero, and then resuming normal operation.

The net effect of rollback or cold-restart on a digital control system is an abrupt change of dynamics. When the control system is linear, the overall system behavior can be modeled as a jump linear system (Tejada et al. 2007), which is described next.

\section{V.2.2 Dynamical Models of Digital Control Systems Deployed in Fault Recoverable Computers}

It was shown in (Tejada et al. 2007, Gray et al. 2000) that the detection and removal of CMF's can be modeled by a two-state Markov chain, $\{\boldsymbol{N}(k), k \geq 0\}$, $\boldsymbol{N}(k) \in\{0,1\}$. This process interacts with the closed-loop system by changing its parameters. Specifically, when no faults are present in the computer, $\boldsymbol{N}(k)=0$, the joint dynamics of the closed-loop system and the recovery mechanism are represented by a nominal state matrix, $A_{0}$. When faults are present, $N(k)=1$, the recovery mechanism is active and the joint dynamics are given by a perturbed state matrix, $A_{1}$. Finally, after the faults are removed, the system returns to its nominal dynamics. Clearly, the overall system behavior can be represented by the following Markov jump 


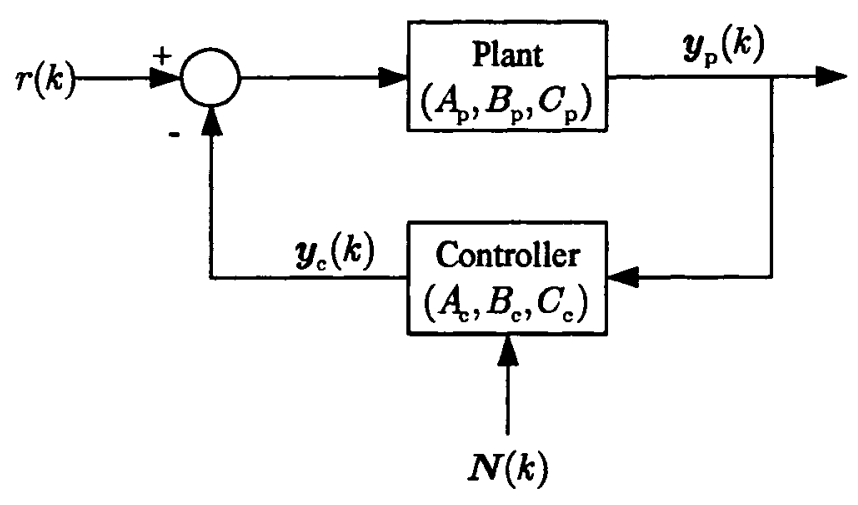

FIG. 15: Digital closed-loop system under consideration.

linear system.

$$
\boldsymbol{x}(k+1)=A_{\boldsymbol{N}(k)} \boldsymbol{x}(k), \quad \boldsymbol{x}(0)=\boldsymbol{x}_{0}
$$

The specific structure of the matrices $A_{0}$ and $A_{1}$ depends on both the dynamics of the closed-loop system and the specific recovery mechanism under consideration. The digital closed-loop system under consideration is shown in Figure 15. The plant and the controller are represented, respectively, by the state space models $\left(A_{\mathrm{p}}, B_{\mathrm{p}}, C_{\mathrm{p}}\right)$ and $\left(A_{\mathrm{c}}, B_{\mathrm{c}}, C_{\mathrm{c}}\right)$. When the reference signal, $r(k)$, is set to zero, the closed-loop dynamics are given by

$$
\boldsymbol{x}_{\mathrm{CL}}(k+1)=A_{\mathrm{CL}} \boldsymbol{x}_{\mathrm{CL}}(k)
$$

where $A_{\mathrm{CL}}=\left[\begin{array}{cc}A_{\mathrm{p}} & -B_{\mathrm{p}} C_{\mathrm{c}} \\ B_{\mathbf{c}} C_{\mathrm{p}} & A_{\mathrm{c}}\end{array}\right]$ and $\boldsymbol{x}_{\mathrm{CL}}(k)=\left[\boldsymbol{x}_{\mathbf{p}}^{\mathrm{T}}(k) \boldsymbol{x}_{\mathbf{c}}^{\mathrm{T}}(k)\right]^{\mathrm{T}} \in \mathbb{R}^{n}$. Here $\boldsymbol{x}_{\mathrm{p}}(k) \in \mathbb{R}^{n_{\mathrm{p}}}$ and $\boldsymbol{x}_{\mathrm{c}}(k) \in \mathbb{R}^{n_{\mathrm{c}}}$ denote, respectively, the plant and the controller state vectors with $n=n_{\mathrm{p}}+n_{\mathrm{c}}$. To capture the effect of the recovery mechanism on the closed-loop system, this state space model must be enlarged to include an extra state vector, $\bar{x}_{\mathbf{r}}(k)$. As explained in (Tejada et al. 2007), the extra state vector is used both to 
store checkpoint information and to enable the reload-and-retry process. The state matrices $A_{0}$ and $A_{1}$ for both rollback and cold-restart recovery, are given below:

$$
\begin{aligned}
& \text { Rollback: } \quad A_{0_{\mathrm{RB}}}=\left[\begin{array}{ccc}
A_{\mathrm{p}} & -B_{\mathrm{p}} C_{\mathrm{c}} & 0 \\
B_{\mathrm{c}} C_{\mathrm{p}} & A_{\mathrm{c}} & 0 \\
0 & I_{n_{\mathrm{c}}} & 0
\end{array}\right], \quad A_{\mathrm{l}_{\mathrm{RB}}}=\left[\begin{array}{ccc}
A_{\mathrm{p}} & 0 & -B_{\mathrm{p}} C_{\mathrm{c}} \\
0 & 0 & I_{n_{\mathrm{c}}} \\
0 & 0 & I_{n_{\mathrm{c}}}
\end{array}\right] \\
& \text { Cold-Restart: } \quad A_{0_{\mathrm{CR}}}=\left[\begin{array}{ccc}
A_{\mathrm{p}} & -B_{\mathrm{p}} C_{\mathrm{c}} & 0 \\
B_{\mathrm{c}} C_{\mathrm{p}} & A_{\mathrm{c}} & 0 \\
0 & I_{n_{\mathrm{c}}} & 0
\end{array}\right], \quad A_{1_{\mathrm{CR}}}=\left[\begin{array}{ccc}
A_{\mathrm{p}} & 0 & -B_{\mathrm{p}} C_{\mathrm{c}} \\
0 & 0 & 0 \\
0 & 0 & I_{n_{\mathrm{c}}}
\end{array}\right] \text {. }
\end{aligned}
$$

Note from (98) that the state vector in (97), $\boldsymbol{x}(k)$, is given by $\boldsymbol{x}(k)=$ $\left[\boldsymbol{x}_{\mathrm{p}}^{\mathrm{T}}(k) \boldsymbol{x}_{\mathrm{c}}^{\mathrm{T}}(k) \overline{\boldsymbol{x}}_{\mathrm{r}}^{\mathrm{T}}(k)\right]^{\mathrm{T}} \in \mathbb{R}^{n_{\mathrm{p}}+2 n_{\mathrm{c}}}$. Also, note that $A_{0_{\mathrm{RB}}}$ and $A_{0_{\mathrm{CR}}}$ are identical.

The MS stability of (97) can be determined through the MS stability test in (Costa et al. 2005). Using this test, it was shown in (Tejada et al. 2007) that rollback usually has better stability properties than cold-restart. It was found that rollback usually remains stable for higher probabilities of faults, that is, for larger values of $\operatorname{Pr}\{\boldsymbol{N}(k)=1 \mid \boldsymbol{N}(k-1)=0\}$ and $\operatorname{Pr}\{\boldsymbol{N}(k)=1 \mid \boldsymbol{N}(k-1)=1\}$. On the other hand, it also requires a more complex hardware implementation and higher power requirements. Thus, in some situations it is better to combine the benefits of both approaches. An example of such a situation is introduced in the next section.

\section{V.3 AN ADVANCED FAULT RECOVERY MECHANISM}

Consider a low-power application such as a satellite or an unmanned aerial vehicle. The lifetime of such high-performance devices is proportional to the availability of electrical power. Thus, their computer platforms should be designed to balance good 
performance and minimal energy consumption. In terms of resilience to CFM's, this tradeoff is addressed by following the strategy:

- To save power, the cold-restart circuits should be active (and the rollback circuits should be powered down) in situations of low CMF's incidence. The converse should be done in high CMF's incidence conditions.

- The rollback circuits should be active (and cold-restart's inactive) when the performance of the closed-loop system is below a prescribed threshold.

The focus of this example is on the second part of this strategy. It is assumed that the powering up and down of the rollback or cold-restart circuits is instantaneous, and that a recovery mechanism cannot be powered down while it is active. The performance metric of interest is the size of the state vector's norm. Thus, the closed-loop system has good performance whenever $\|\boldsymbol{x}(k)\|<\alpha$, where $\alpha$ is a fixed performance boundary. In the absence of faults, the nominal dynamics are given by $A_{0_{\mathrm{RB}}}=A_{0_{\mathrm{CR}}}$. In the presence of faults, the system's dynamics are given by $A_{\mathbf{l}_{\mathrm{CR}}}$ when the performance is good and by $A_{\mathrm{l}_{\mathrm{RB}}}$ when it is bad. Clearly, the supervisor takes decisions based on information from the closed-loop system. Thus, the overall system behavior can be modeled with the following canonical HJLS:

$$
\begin{aligned}
& \boldsymbol{x}(k+1)=A_{\boldsymbol{z}(k)} \boldsymbol{x}(k), \quad \boldsymbol{x}(0)=\boldsymbol{x}_{0} \\
& \boldsymbol{z}(k+1)=S_{\boldsymbol{N}(k), \boldsymbol{\nu}(k)} \boldsymbol{z}(k),
\end{aligned}
$$


where $\boldsymbol{x}(k)=\left[\boldsymbol{x}_{\mathrm{p}}^{\mathrm{T}}(k) \boldsymbol{x}_{\mathrm{c}}^{\mathrm{T}}(k) \overline{\boldsymbol{x}}_{\mathrm{r}}^{\mathrm{T}}(k)\right]^{\mathrm{T}}, A_{\boldsymbol{z}(k)}$ is given by

$$
A_{\boldsymbol{z}(k)}= \begin{cases}A_{0_{\mathrm{RB}}}=A_{0_{\mathrm{CR}}}, & \text { when } \boldsymbol{z}(k)=e_{1} \\ A_{1_{\mathrm{CR}}}, & \text { when } \boldsymbol{z}(k)=e_{2} \\ A_{1_{\mathrm{RB}}}, & \text { when } \boldsymbol{z}(k)=e_{3}\end{cases}
$$

$\boldsymbol{\nu}(k)$ is the output of the $A / S$ map given by

$$
\boldsymbol{\nu}(k)=\psi(\boldsymbol{x}(k))=\mathbf{1}_{\{\|\boldsymbol{x}(k)\| \geq \alpha\}},
$$

and the structure of the supervisor's FSM is given by the state transition diagram in Figure 16. In this Figure, the circles represent the current state of the FSM, $z(k)$. They also represent the current output symbol since $\boldsymbol{\theta}(k)=\boldsymbol{z}(k)$. The arcs between the circles represent the valid state transitions. The labels on the arcs indicate the value of the FSM's input pair, $(N(k), \nu(k))$, that enable such transition (the $x$ represents a don't care condition). Note that $\nu(k)=0$ whenever the performance is 'good' and $\boldsymbol{\nu}(k)=1$ whenever it is 'bad'. Hence, the FSM remains in state $e_{1}$ whenever no CMF's are detected. Upon their detection, $N(k)=1$, the FSM transitions to state $e_{2}$ or $e_{3}$ depending on, respectively, the $\operatorname{good}(\boldsymbol{\nu}(k)=0)$ or bad $(\boldsymbol{\nu}(k)=1)$ closed-loop system performance. The FSM remains in state $e_{2}$ (or $e_{3}$ ) while the effect of the faults is removed and returns to state $e_{1}$ after the recovery is finished.

Note that $\mathcal{I}_{\ell_{N}}=\mathcal{I}_{\ell_{\nu}}=\{0,1\}$ and $\Sigma_{S}=\Sigma_{O}=\left\{e_{1}, e_{2}, e_{3}\right\}$, where $e_{i} \in \mathbb{R}^{3}$ for $i=1,2,3$. It is easy to show that the FSM's state transition diagram in Figure 16 is equivalent to the state evolution equation in (99) provided that the state transition 


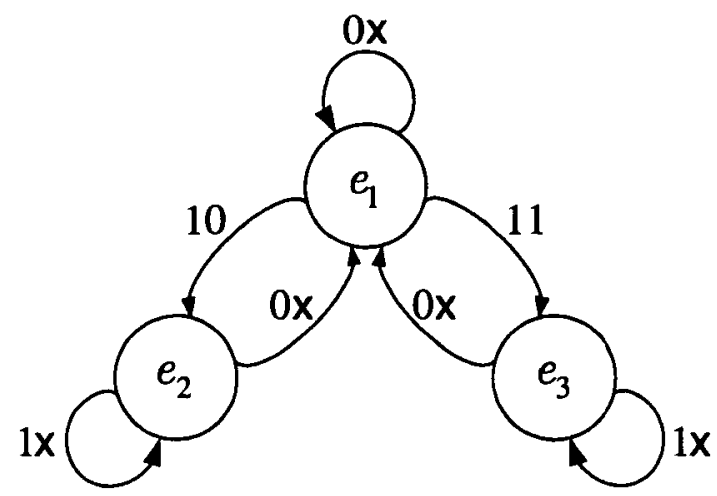

FIG. 16: The example's FSM structure. Inside each circle, $e_{i}$ represents the current state and output symbol. The labels over the arcs represent the value of the FSM's inputs, $(\boldsymbol{N}(k), \boldsymbol{\nu}(k))$, that triggers the state transition (the $\mathrm{x}$ represents a don't care condition).

matrices are given by

$$
S_{0,0}=S_{0,1}=\left[\begin{array}{lll}
1 & 1 & 1 \\
0 & 0 & 0 \\
0 & 0 & 0
\end{array}\right], \quad S_{1,0}=\left[\begin{array}{lll}
0 & 0 & 0 \\
1 & 1 & 0 \\
0 & 0 & 1
\end{array}\right], \quad S_{1,1}=\left[\begin{array}{lll}
0 & 0 & 0 \\
0 & 1 & 0 \\
1 & 0 & 1
\end{array}\right]
$$

Equations (99)-(101) completely determine the HJLS's parameters for this example.

The next section studies the stability of this example.

\section{V.4 STABILITY ANALYSIS OF THE ADVANCED RECOVERY MECHANISM}

\section{V.4.1 Theoretical Stability Analysis}

To simplify the analysis, $\{N(k), k \geq 0\}$ is assumed to be an i.i.d. process with initial distribution $\left[p_{0}^{N} p_{1}^{N}\right]$, where $p_{0}^{N}=\operatorname{Pr}\{N(k)=0\}, p_{1}^{N}=\operatorname{Pr}\{N(k)=1\}$, and transition probability matrix $\Pi_{N}=\left[\begin{array}{cc}p_{0}^{N} & p_{1}^{N} \\ p_{0}^{N} & p_{1}^{N}\end{array}\right]$. Theorem IV.4.2 provides a sufficient mean square stability test for the HJLS in (99)-(101). The test requires computing 
$\rho\left(\mathcal{A}_{M_{z}}\right)$, where

$$
\mathcal{A}_{M_{z}}=\left(M_{z}^{\mathrm{T}} \otimes I_{n^{2}}\right) \operatorname{diag}\left(A_{1} \otimes A_{1}, \ldots, A_{3} \otimes A_{3}\right)
$$

In this example, $A_{1}=A_{0_{\mathrm{CR}}}=A_{0_{\mathrm{RB}}}, A_{2}=A_{1_{\mathrm{CR}}}, A_{3}=A_{1_{\mathrm{RB}}}$, and $M_{z}=\left[M_{r, s}\right]$ is given by (see (77))

$$
M_{z}=\left[\begin{array}{ccc}
p_{0}^{N} & p_{1}^{N} & p_{1}^{N} \\
p_{0}^{N} & p_{1}^{N} & 0 \\
p_{0}^{N} & 0 & p_{1}^{N}
\end{array}\right]
$$

Note that computing $\rho\left(\mathcal{A}_{M_{z}}\right)$ takes into consideration the structure of the FSM and the probabilities associated with of $\{N(k), k \geq 0\}$. It also contains information pertaining to the jump linear closed-loop system. It does not, however, take into account the $A / S$ map. More specifically, it does not take into account the parameter $\alpha$, which plays a significant role in the behavior of the HJLS. To see this, observe that $\alpha$ divides the closed-loop system's phase space into two regions: $R_{0}=\left\{x \in \mathbb{R}^{n}\right.$ : $\|x\|<\alpha\}$ and $R_{1}=\left\{x \in \mathbb{R}^{n}:\|x\| \geq \alpha\right\}$. When $\alpha=\infty, R_{1}$ is empty and $x(k) \in R_{0}$ for all $k \geq 0$. This in turn implies that $\nu(k)=0$ for all $k \geq 0$, which restricts the supervisor's operation to only states $e_{1}$ and $e_{2}$. That is, the ARM behaves as a simple cold-restart recovery mechanism. On the other hand, when $\alpha=0, R_{0}$ is empty and $\boldsymbol{\nu}(k)=1$ for all $k \geq 0$. Thus, the supervisor only commutes between states $e_{1}$ and $e_{3}$, and the ARM behaves as a simple rollback recovery mechanism. These observations can be formalized using Theorem IV.4.1. For instance, when $\alpha=\infty, R_{0}$ becomes a common invariant subspace (clearly, $\boldsymbol{x}_{0} \in R_{0}=\mathbb{R}^{n}$ ). This implies that the HJLS behaves as a JLS driven by a FSM (see Figure 3) whose behavior is given by the state transition diagram in Figure 17. In this case, the HJLS's mean square stability can 


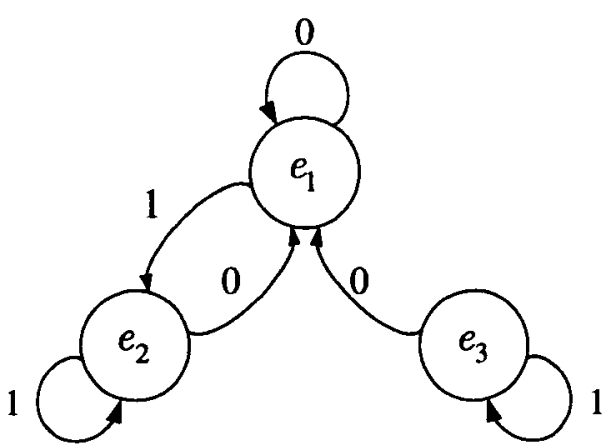

FIG. 17: The restricted operation of the supervisor when $\alpha=\infty$. The labels over the arcs show the value of $\boldsymbol{N}(k)(\boldsymbol{\nu}(k)=0$ for all $k \geq 0$ ).

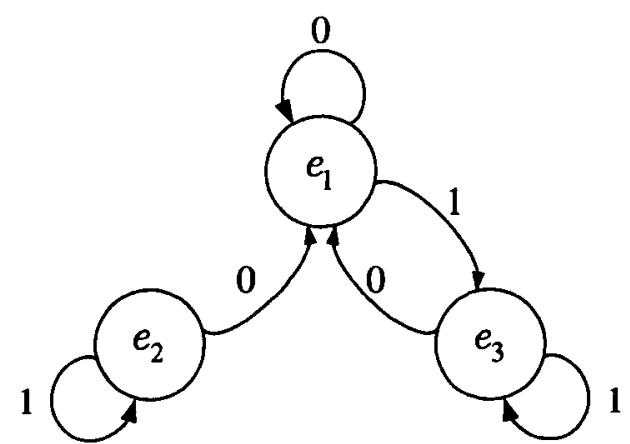

FIG. 18: The restricted operation of the supervisor when $\alpha=0$. The labels over the arcs show the value of $N(k)(\nu(k)=1$ for all $k \geq 0$ ).

be determined by computing $\rho\left(A_{i i d_{\mathrm{CR}}}\right)$, where

$$
\mathcal{A}_{i i d_{\mathrm{CR}}}=\left(\Pi_{z_{\mathrm{CR}}}^{\mathrm{T}} \otimes I_{n^{2}}\right) \operatorname{diag}\left(A_{0_{\mathrm{CR}}} \otimes A_{0_{\mathrm{CR}}}, A_{1_{\mathrm{CR}}} \otimes A_{1_{\mathrm{CR}}}, A_{1_{\mathrm{RB}}} \otimes A_{1_{\mathrm{RB}}}\right)
$$

with $\Pi_{z_{\mathrm{CR}}}=\left[\begin{array}{ccc}p_{0}^{N} & p_{1}^{N} & 0 \\ p_{0}^{N} & p_{1}^{N} & 0 \\ p_{0}^{N} & 0 & p_{1}^{N}\end{array}\right]$. That is,

$$
\mathcal{A}_{i i d_{\mathrm{CR}}}=\left[\begin{array}{ccc}
p_{0}^{N} A_{0_{\mathrm{CR}}} \otimes A_{0_{\mathrm{CR}}} & p_{0}^{N} A_{1_{\mathrm{CR}}} \otimes A_{1_{\mathrm{CR}}} & p_{0}^{N} A_{1_{\mathrm{RB}}} \otimes A_{1_{\mathrm{RB}}} \\
p_{1}^{N} A_{0_{\mathrm{CR}}} \otimes A_{0_{\mathrm{CR}}} & p_{1}^{N} A_{1_{\mathrm{CR}}} \otimes A_{1_{\mathrm{CR}}} & 0 \\
0 & 0 & p_{1}^{N} A_{1_{\mathrm{RB}}} \otimes A_{1_{\mathrm{RB}}}
\end{array}\right]
$$

which in turn implies that

$$
\rho\left(\mathcal{A}_{i i d_{\mathrm{CR}}}\right)=\max \left\{\rho\left(\begin{array}{ll}
p_{0}^{N} A_{0_{\mathrm{CR}}} \otimes A_{0_{\mathrm{CR}}} & p_{0}^{N} A_{1_{\mathrm{CR}}} \otimes A_{1_{\mathrm{CR}}} \\
p_{1}^{N} A_{0_{\mathrm{CR}}} \otimes A_{0_{\mathrm{CR}}} & p_{1}^{N} A_{1_{\mathrm{CR}}} \otimes A_{1_{\mathrm{CR}}}
\end{array}\right), \rho\left(p_{1}^{N} A_{1_{\mathrm{RB}}} \otimes A_{1_{\mathrm{RB}}}\right)\right\} .
$$

Let $\mathcal{A}_{\mathrm{CR}}=\left[\begin{array}{ll}p_{0}^{N} A_{0_{\mathrm{CR}}} \otimes A_{0_{\mathrm{CR}}} & p_{0}^{N} A_{\mathrm{l}_{\mathrm{CR}}} \otimes A_{1_{\mathrm{CR}}} \\ p_{1}^{N} A_{0_{\mathrm{CR}}} \otimes A_{0_{\mathrm{CR}}} & p_{1}^{N} A_{\mathbf{l}_{\mathrm{CR}}} \otimes A_{\mathrm{I}_{\mathrm{CR}}}\end{array}\right]$. By Corollary 3.11 in (Tejada et al. 2007), $\rho\left(\mathcal{A}_{\mathrm{CR}}\right)$ characterizes the mean square stability of a digital closed-loop system run by a computer platform equipped only with cold-restart. Hence, it follows that when $\alpha=\infty$ and the probability of faults is small, the mean square stability of the HJLS is similar to that of a system equipped with cold restart only. 
When $\alpha=0$. the HJLS's mean square stability can be determined by computing $\rho\left(A_{i i d_{\mathrm{RB}}}\right)$, where

$$
\mathcal{A}_{i i d_{\mathrm{RB}}}=\left[\begin{array}{ccc}
p_{0}^{N} A_{0_{\mathrm{RB}}} \otimes A_{0_{\mathrm{RB}}} & p_{0}^{N} A_{1_{\mathrm{CR}}} \otimes A_{1_{\mathrm{CR}}} & p_{0}^{N} A_{\mathbf{1}_{\mathrm{RB}}} \otimes A_{1_{\mathrm{RB}}} \\
0 & p_{1}^{N} A_{\mathbf{1}_{\mathrm{CR}}} \otimes A_{1_{\mathrm{CR}}} & p_{1}^{N} A_{\mathbf{1}_{\mathrm{RB}}} \otimes A_{1_{\mathrm{RB}}} \\
p_{1}^{N} A_{0_{\mathrm{RB}}} \otimes A_{0_{\mathrm{RB}}} & 0 & p_{1}^{N} A_{\mathbf{1}_{\mathrm{RB}}} \otimes A_{1_{\mathrm{RB}}}
\end{array}\right] .
$$

It is easy to show that in this case

$$
\rho\left(\mathcal{A}_{i i d_{\mathrm{RB}}}\right)=\max \left\{\rho\left(\begin{array}{ll}
p_{0}^{N} A_{0_{\mathrm{RB}}} \otimes A_{0_{\mathrm{RB}}} & p_{0}^{N} A_{1_{\mathrm{RB}}} \otimes A_{1_{\mathrm{RB}}} \\
p_{1}^{N} A_{0_{\mathrm{RB}}} \otimes A_{0_{\mathrm{RB}}} & p_{1}^{N} A_{1_{\mathrm{RB}}} \otimes A_{1_{\mathrm{CR}}}
\end{array}\right), \rho\left(p_{1}^{N} A_{1_{\mathrm{CR}}} \otimes A_{1_{\mathrm{CR}}}\right)\right\} .
$$

Again, let $\mathcal{A}_{\mathrm{RB}}=\left[\begin{array}{ll}p_{0}^{N} A_{0_{\mathrm{RB}}} \otimes A_{0_{\mathrm{RB}}} & p_{0}^{N} A_{1_{\mathrm{RB}}} \otimes A_{1_{\mathrm{RB}}} \\ p_{1}^{N} A_{0_{\mathrm{RB}}} \otimes A_{0_{\mathrm{RB}}} & p_{1}^{N} A_{1_{\mathrm{RB}}} \otimes A_{1_{\mathrm{RB}}}\end{array}\right]$, and observe that if the probability of faults is small then $\rho\left(\mathcal{A}_{i i i_{\mathrm{RB}}}\right) \approx \rho\left(\mathcal{A}_{\mathrm{RB}}\right)$. In this case, the HJLS's mean square stability is equivalent to that of a system equipped with rollback only.

Finally, observe that whenever $\alpha=0$ or $\alpha=\infty$, the process $\{z(k), k \geq 0\}$ becomes a Markov chain because $\{N(k), k \geq 0\}$ is an i.i.d. process (see Lemma IV.4.1). Thus, the HJLS behaves as a MJLS, and the logarithm of its second moment approaches a linear asymptote (Tejada et al. 2007). That is,

$$
\begin{array}{ll}
\lim _{k \rightarrow \infty} \log _{10}\left(\mathbf{E}\left\{\|x(k)\|^{2}\right\}\right) \approx k \log _{10}\left(\rho\left(\mathcal{A}_{i i d_{\mathrm{CR}}}\right)\right) & (\alpha=\infty) \\
\lim _{k \rightarrow \infty} \log _{10}\left(\mathbf{E}\left\{\|\boldsymbol{x}(k)\|^{2}\right\}\right) \approx k \log _{10}\left(\rho\left(\mathcal{A}_{i i d_{\mathrm{RB}}}\right)\right) & (\alpha=0)
\end{array}
$$

As explained in (Tejada et al. 2007), the intercepts for these asymptotes can be fixed arbitrarily. In light of these comments, it is expected that as $\alpha$ varies from 0 to $\infty$, the limit behavior of $\log _{10}\left(E\left\{\|x(k)\|^{2}\right\}\right)$ should vary from $k \log _{10}\left(\rho\left(\mathcal{A}_{i i d_{\mathbf{R B}}}\right)\right)$ to $k \log _{10}\left(\rho\left(\mathcal{A}_{i d_{\mathrm{CR}}}\right)\right)$. Also, $\rho\left(\mathcal{A}_{\mathrm{RB}}\right)$ usually remains below 1 for larger values of $p_{0}^{N}$ than $\rho\left(\mathcal{A}_{\mathrm{CR}}\right)$. Thus, for a large enough $p_{0}^{N}$ the HJLS should be MS stable for $\alpha=0$ and unstable for $\alpha=\infty$. This observation suggests that $\rho\left(\mathcal{A}_{M_{z}}\right)<1$ only when both $\rho\left(\mathcal{A}_{i i d_{\mathrm{CR}}}\right)<1$ and $\rho\left(\mathcal{A}_{i i d_{\mathrm{RB}}}\right)<1$. These conclusions, however, cannot be drawn from 


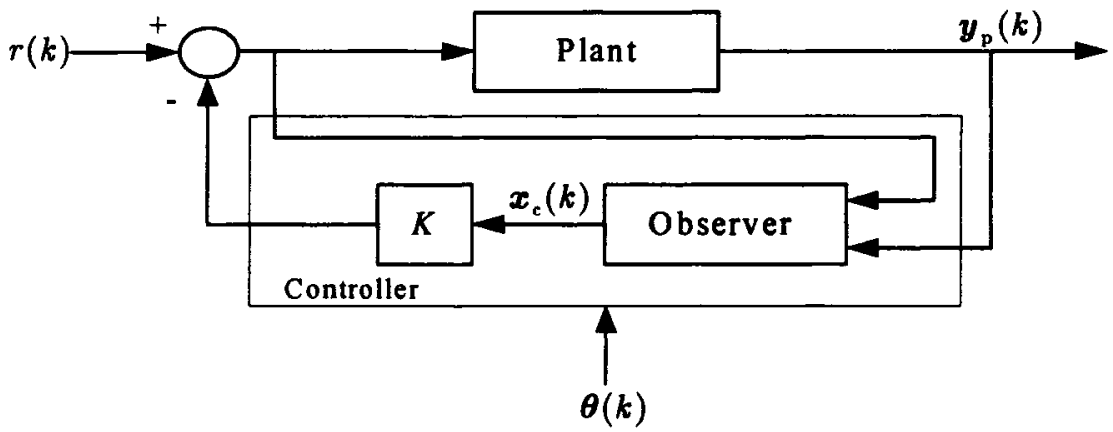

FIG. 19: Digital jump linear closed-loop system considered for Monte Carlo simulations.

the theory introduced in Chapter IV. Nevertheless, these conclusions were confirmed through Monte Carlo simulations as explained next.

\section{V.4.2 Monte Carlo Simulations}

Figure 19 shows the specific system considered for numerical analysis. The plant corresponds to the sampled data equivalent of the longitudinal dynamics of an AFTIF16 aircraft (Friedland 1986). The controller is an observer-based regulator deployed in a computer equipped with the ARM. The data for this example was taken from (Tejada et al. 2007). The observer-based digital regulator dynamics are given by

$$
\begin{aligned}
\boldsymbol{x}_{\mathrm{c}}(k+1) & =A_{\mathrm{p}} \boldsymbol{x}_{\mathrm{c}}(k)+B_{\mathrm{p}} \boldsymbol{u}(k)+L\left(\boldsymbol{y}_{\mathrm{p}}-C_{\mathrm{p}} \boldsymbol{x}_{\mathrm{c}}\right) \\
\boldsymbol{y}_{\mathrm{c}}(k) & =\boldsymbol{x}_{\mathrm{c}}(k),
\end{aligned}
$$

where $\boldsymbol{u}(k)=r(k)-K \boldsymbol{x}_{\mathrm{c}}(k)$, and $L$ is the output injection matrix. The nominal closed-loop discrete-time dynamics are given by

$$
A_{\mathrm{CL}}=\left[\begin{array}{cc}
A_{\mathrm{p}} & -B_{\mathrm{p}} K \\
-L C_{\mathrm{p}} & A_{\mathrm{p}}-B_{\mathrm{p}} K-L C_{\mathrm{p}}
\end{array}\right],
$$


where $K$ is the state feedback (pole-placement) matrix. The sampling period was set to $T=0.004$ sec., the nominal continuous-time closed-loop poles were placed at $\{-0.2 \pm j 0.9798,-0.01 \pm j 0.0995\}$, and the observer's discrete-time poles were chosen to be five times faster than the plant's closed-loop poles.

The spectral radii $\rho\left(\mathcal{A}_{M_{z}}\right), \rho\left(\mathcal{A}_{i d_{\mathrm{CR}}}\right)$, and $\rho\left(\mathcal{A}_{i i d_{\mathrm{RB}}}\right)$ were computed for several values of $\mathrm{p}_{0}^{N}=\operatorname{Pr}\{\boldsymbol{N}(k)=0\}$. The radii are shown in Figure 20. As expected, the HJLS equipped with 'only' rollback $(\alpha=0)$ behaves better than when it is equipped with 'only' cold-restart $(\alpha=\infty)$. That is, $\rho\left(\mathcal{A}_{i i d_{\mathrm{RB}}}\right)$ remains below 1 for a wider range of values of $p_{0}^{N}$ than $\rho\left(\mathcal{A}_{i i d_{\mathrm{CR}}}\right)$. When $\alpha \in(0, \infty)$, the stability of the system can be assessed by testing if $\rho\left(\mathcal{A}_{M_{z}}\right)<1$. In this case, the bottom plot in Figure 20 shows that the HJLS is stable when $\operatorname{Pr}\{\boldsymbol{N}(k)=0\}>0.9999$.

Two Monte Carlo simulations of the HJLS were performed. Each simulation comprised 500 runs of 1000 seconds (250,000 samples). In both cases, $p_{0}^{N}=0.99994$, $z_{0}=e_{1}$, and $x_{0}=\left[\begin{array}{llllllllllll}0 & 1 & 0 & 0 & 0 & 0 & 0 & 0 & 0 & 0 & 0 & 0\end{array}\right]^{\mathrm{T}}$ (angle of attack perturbed by 1 radian (Tejada et al. 2007)). The top plot presents the simulation results for $\alpha=0$, while the bottom plot shows the simulation results for $\alpha=1000$ (both in blue). The linear fit of the simulation data was also plotted (black). As expected, the linear fit of the simulation data closely approximates the asymptotes (red) given by $3.7172+t \log _{10}\left(\rho\left(\mathcal{A}_{i i d_{\mathrm{RB}}}\right)\right)$ (top) and $4.4841+t \log _{10}\left(\rho\left(\mathcal{A}_{i i d_{\mathrm{CR}}}\right)\right)$ (bottom). Figure 21 shows that the linear fit and the asymptote nearly overlap. Hence, in both cases the simulation of the HJLS behaved as expected. 

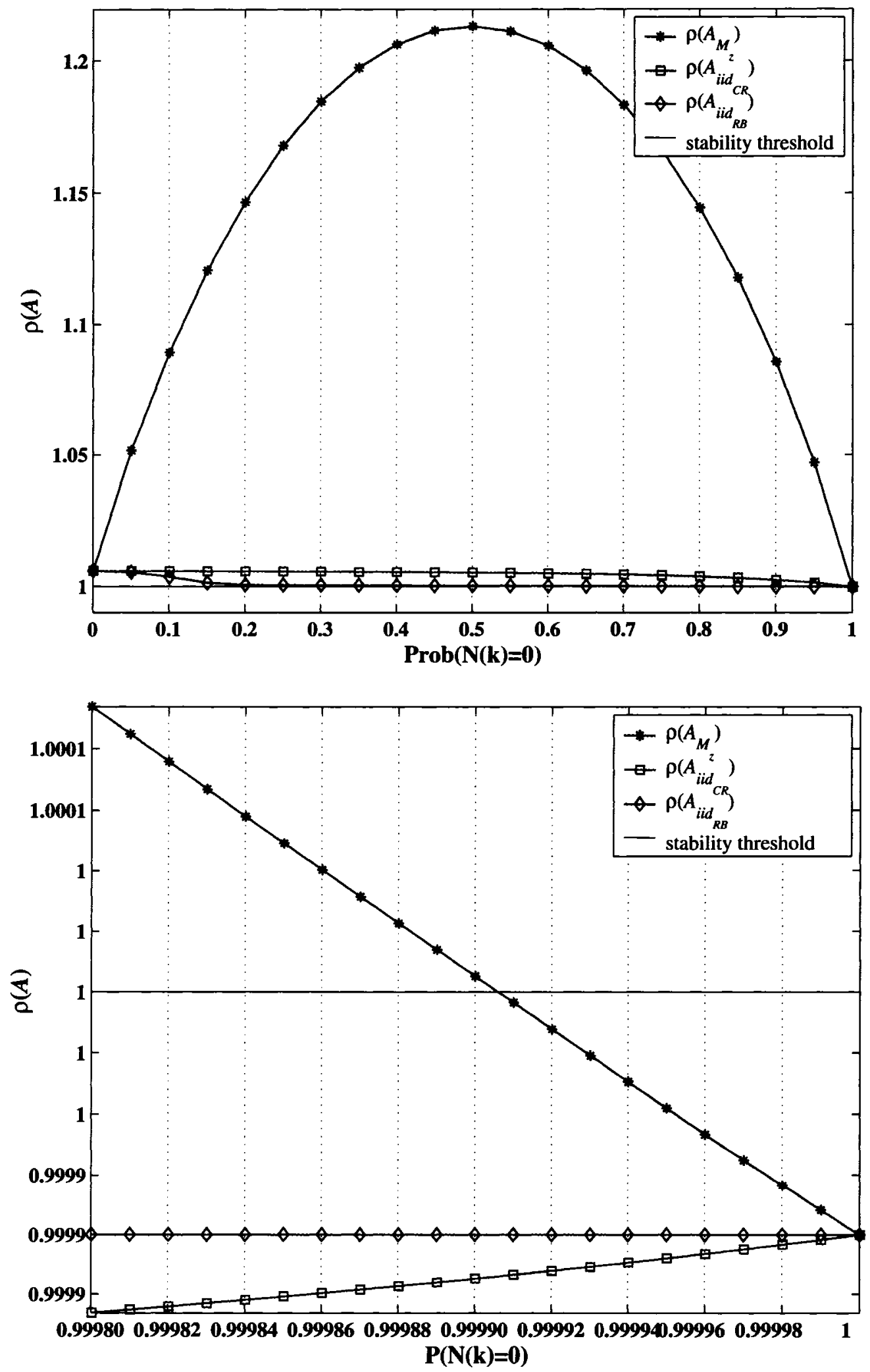

FIG. 20: Top: Spectral radius of $\mathcal{A}_{M_{z}}, \mathcal{A}_{\text {iid }}$, and $\mathcal{A}_{\text {iid }}$ RB as a function of $p_{0}^{N}=$ $\operatorname{Pr}\{N(k)=0\}$. Bottom: A magnification of the region $0.9998 \leq p_{0}^{N} \leq 1$. 

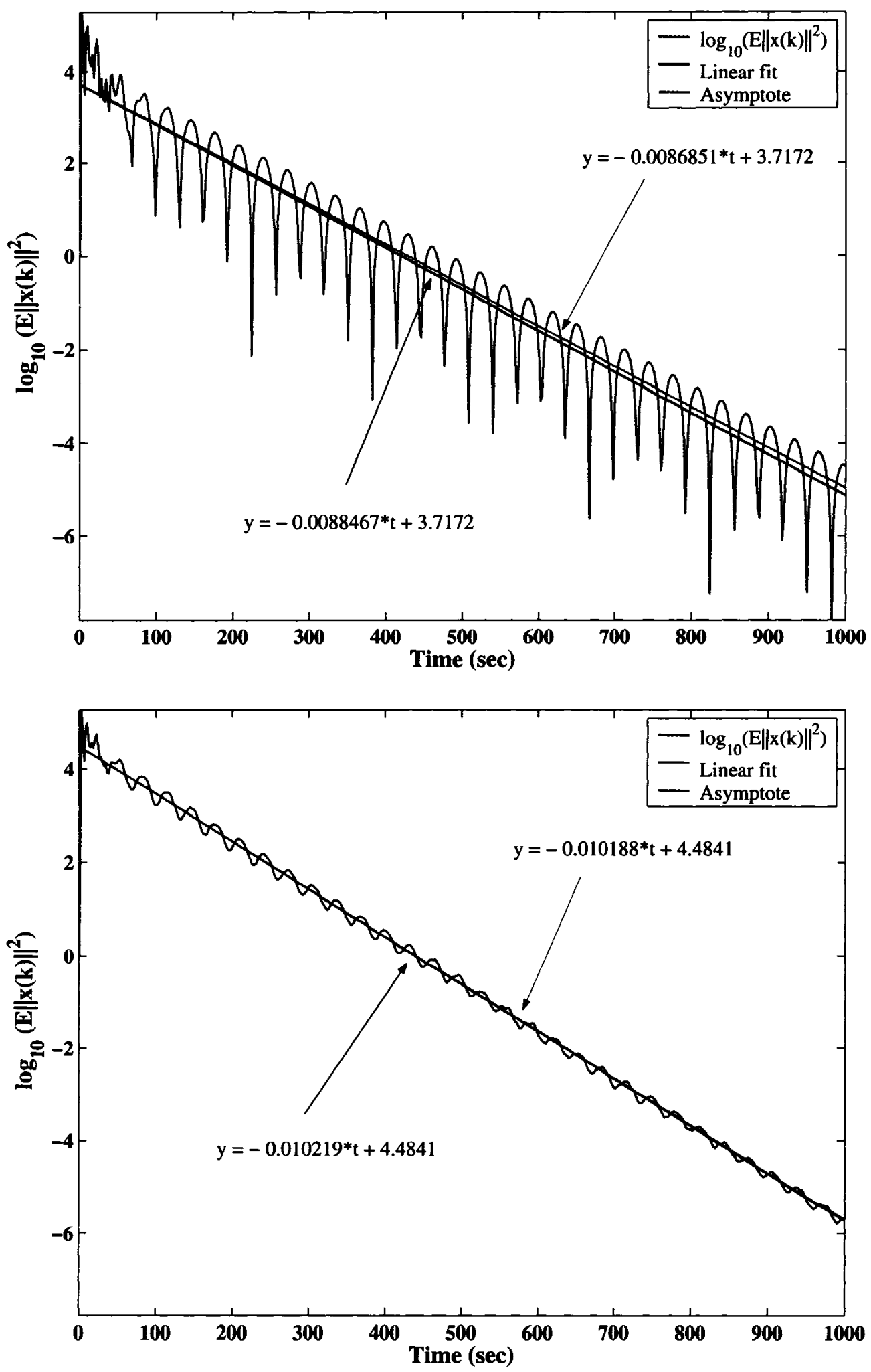

FIG. 21: Monte Carlo simulation results. Each simulation comprised 500 runs of 1000

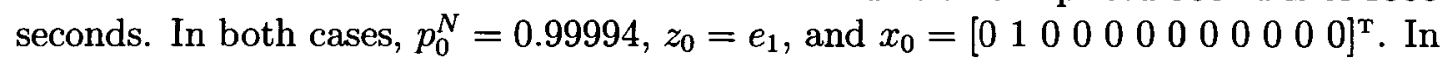
the top plot $\alpha=0$, while in the bottom plot $\alpha=1000$. 


\section{CHAPTER VI}

\section{CONCLUSIONS AND FUTURE RESEARCH}

\section{VI.1 CONCLUSIONS}

The main contributions of this dissertation are the introduction of hybrid jump linear systems as a relevant class of discrete-time, stochastic hybrid systems; a variety of stability analysis methods for this class; and the application of HJLS's to study a real-world example: an AFTI-F16 digital flight controller deployed on a fault tolerant controller.

It was shown in Chapters II and V that hybrid jump linear systems constitute a suitable framework to study systems which combine both continuous and symbolic dynamics. Through this framework, it is possible to model and analyze the behavior of complex supervisory control systems and other hybrid systems of practical interest.

Chapter III introduced a suitable stability analysis framework for HJLS's. Although this framework is not entirely new (see Hou \& Michel 2001b), it was the first time this framework was employed to develop testable sufficient mean square stability conditions for discrete-time stochastic hybrid systems. A number of sufficient stability tests were introduced. The most important results are listed at the end of Chapter III. It was also shown that some of these tests can be applied to general discrete-time switched systems.

The use of Markov kernel techniques to study discrete-time stochastic hybrid systems constitutes an entirely new approach. It was shown in Chapter IV, that 
the state processes of HJLS's constitute Markov chains. Consequently, the Markov kernels of these state processes can be used to deduce the stability characteristics of the associated HJLS's. The techniques and concepts used to derive the results in Theorems IV.4.2 and IV.4.4 can also be used to establish the ergodicity of HJLS's. This in turn could lead to less conservative stability conditions or the study of the stability of HJLS's through Monte Carlo simulations.

The stability conditions in Theorem IV.4.4 can be extended to a larger class of HJLS's. Such an extension, however, would require significant algebraic manipulations and would not contribute any new concepts or techniques. Therefore, it has not been included in this dissertation.

The ability of HJLS's to model and study real-world applications was demonstrated through the analysis of the effects of an advanced recovery mechanisms on the stability of digital closed-loop systems. An AFTI-F16 example was presented. In this example, the aircraft's digital flight controller was assumed to be deployed in a fault tolerant computer subject to a harsh environment. The computer was equipped with a novel advanced recovery system, which selects the best fault recovery procedure based on the performance of the closed-loop system. The analysis of such a recovery scheme was not possible with the tools currently available in the literature.

\section{VI.2 FUTURE RESEARCH}

The body of work presented in this documents can be extended in many directions.

- Although the stability conditions in Theorems IV.4.2 and IV.4.4 include information about the number of quantization regions created by $\psi$ (see Chapter 
II), the relative size of these regions is not taken into consideration. More simulation experiments are necessary to assess, for example, if the probability that the closed-loop system's state vector visits certain quantization region is proportional to the relative size of this region. A theoretical characterization may be also possible using more advanced tools for analyzing Markov chains.

- The relation between HJLS (or DSHA) and discrete-time PDP's suggested by A. Bemporad and coauthors (Bemporad \& Di Cairano 2005) needs to be formalized. This would enable one to study discrete-time PDPs with the tools introduced here and to study HJLS with the tools available for PDPs.

- The ergodicity of HJLS needs to be explored. It is known that when a Markov chain in a metric space is mean square stable, it is also weakly convergent and ergodic (Hernández-Lerma \& Lasserre 2003). Since the stability of any ergodic system can be studied through Monte Carlo simulations, it is important to characterize the conditions under which a HJLS is ergodic (mean square stability is a sufficient but not a necessary condition).

- The connection between DSHA and HJLS's needs to be further studied. A DSHA can be translated into the MLD formalism and formally verified. In principle, this implies that HJLS's can also be formally verified. This, however, needs to be studied further since HJLS's are not deterministic but stochastic models. 


\section{BIBLIOGRAPHY}

Antsaklis, P., Koutsoukos, X. \& Zaytoon, J. (1998), 'On hybrid control of complex systems: A survey', Eur. J. Automat 32(9-10), 1023-1045.

Arnold, L. (1974), Stochastic Differential Equations: Theory and Applications, John Wiley \& Sons, New York.

Arnold, L. \& Kleinmann, W. (1982), Qualitative theory of stochastic systems, in A. T. Bharucha-Reid, ed., 'Probabilistic Analysis and Related Topics, Vol 3', Academic Press, New York, pp. 1-79.

Belcastro, C. M. (1997), Closed-loop HIRF experiments performed on a fault tolerant flight control computer, in 'Proc. of the 16th DASC Digital Avionics Systems Conference', Philadelphia, PA, pp. 4.1-40-54.

Bemporad, A. \& Di Cairano, S. (2005), Optimal control of discrete hybrid stochastic automata, in M. Morari \& L. Thiele, eds, 'Hybrid Systems: Computation and Control HSCC 2005', Lecture Notes in Computer Science 3414, Springer Verlag, pp. 151-167.

Bemporad, A. \& Morari, M. (1999), 'Control of systems integrating logic, dynamics, and constraints', Automatica 35(3), 407-427.

Billingsley, P. (1995), Probability and Measure, Third Edition, John Wiley \& Sons, New York. 
Costa, O. L. V. \& Fragoso, M. D. (1993), 'Stability results for discrete-time linear systems with Markovian jumping parameters', Mathematical Analysis and Applications 179, 154-178.

Costa, O. L. V., Fragoso, M. D. \& Marques, R. P. (2005), Discrete-Time Markov Jump Linear Systems, Springer, London.

Daafouz, J., Riedinger, P. \& Iung, C. (2002), 'Stability analysis and control synthesis for switched systems: A switched Lyapunov function approach', IEEE Trans. Automat. Contr. 47(11), 1883-1887.

Davis, M. H. A. (1993), Markov Models and Optimization, Chapman \& Hall/CRC, New York.

DeCarlo, R. A., Branicky, M. S., Petterson, S. \& Lennartson, B. (2000), 'Perspectives and results on the stability and stabilizability of hybrid systems', Proceedings of the IEEE 88(7), 1069-1082.

Ehrhardt, M. \& Kleinman, W. (1982), 'Controllability of linear stochastic systems', Systems $\&$ Control Letters 2(3), 145-153.

Fang, Y. \& Loparo, K. A. (2002), 'Stochastic stability of jump linear systems', IEEE Trans. Automat. Contr. 47(7), 1204-1208.

Fang, Y., Loparo, K. A. \& Feng, X. (1995), 'Stability of discrete time jump linear systems', Journal of Mathematical Systems, Estimation, and Control 5(3), 275321. 
Ferrari-Trecate, G., Cuzzola, F. A. \& Morari, M. (2003), 'Lagrange stability and performance analysis of discrete-time piecewise affine systems with logic states', Int. J. Control 76(16), 1585-1598.

Friedland, B. (1986), Control System Design, An Introduction to State-Space Methods, McGraw-Hill, New York, NY.

Friedman, A. (1982), Foundations of Modern Analysis, Dover Publications Inc., New York, NY.

Gray, W. S., González, O. R. \& Dǒgan, M. (2000), 'Stability analysis of digital linear flight controllers subject to electromagnetic disturbances', IEEE Trans. Aerosp. Electron. Syst. 36(4), 1204-1218.

Gurvits, L. (1995), 'Stability of discrete linear inclusion', Linear Algebra and its Applications 231(1), 47-85.

Hassibi, A., Boyd, S. P. \& How, J. P. (1999), A class of Lyapunov functionals for analyzing hybrid dynamical systems, in 'Proc. of the 1999 American Control Conference', Arlington, VA, pp. 2455-2460.

Heemels, W. P. M. H., Schutter, B. D. \& Bemporad, A. (2001), 'Equivalence of hybrid dynamical models', Automatica 37(7), 1085-1091.

Henzinger, T. A. (1996), The theory of hybrid automata, in 'Proc. of the 11th Annual Symposium on Logic in Computer Science', New Brunswick, NJ, pp. 278-292.

Hernández-Lerma, O. \& Lasserre, J. B. (2003), Markov Chains and Invariant Probabilities, Birkhäuser Verlag, Boston, MA. 
Hess, R. (1997), Computing platform architectures for robust operation in the presence of lightning and other electromagnetic threats, in '16th DASC Digital Avionics Systems Conference', Philadelphia PA, pp. 4.3-9.16.

Hess, R. \& Belcastro, C. M. (2001), Design and verification of robust architectures for electronic systems, in 'Proc. of the 2001 International Conference on Lightning and Static Electricity', Seattle, WA.

Hou, L. \& Michel, A. N. (2001a), 'Moment stability of discontinuous stochastic dynamical systems', IEEE Trans. Automat. Contr. 46(6), 938-943.

Hou, L. \& Michel, A. N. (2001b), 'Stability preserving mappings for stochastic dynamical systems', IEEE Trans. Automat. Contr. 46(6), 933-938.

Ji, Y. \& Chizeck, H. J. (1990a), 'Controllability, stabilizability, and contininuoustime Markovian jump linear quadratic control', IEEE Trans. Automat. Contr. $\mathbf{A C - 3 5}(7), 777-788$.

Ji, Y. \& Chizeck, H. J. (1990b), 'Jump linear quadratic Gaussian control: steady state solution and testable conditions', Control Theory Adv. Tech. 6(3), 289-319.

Ji, Y., Chizeck, J., Feng, X. \& Loparo, K. A. (1991), 'Stability and control of discretetime jump linear systems', Control-Theory and Advanced Technology 7(2), 247270.

Johnson, B. (1989), Design and Analysis of Fault-Tolerant Digital Systems, Addison Wesley Publishing Co, Reading, MA. 
Koutsoukos, X., Antsaklis, P. J., Stiver, J. A. \& Lemmon, M. D. (2000), 'Supervisory control of hybrid systems', Proceedings of the IEEE 88(7), 1026-1049.

Kubrusly, C. S. \& Costa, O. L. V. (1985), 'Mean square stability conditions for discrete stochastic bilinear systems', IEEE Trans. Automat. Contr. 30(11), 1082-1087.

LaSalle, J. P. \& Lefschetz, S. (1961), Stability by Lyapunov's Direct Method with Applications, Academic Press, New York, NY.

Liberzon, D., Hespanha, J. \& Morse, S. (1995), 'Stability of switched systems: a Lie-algebraic condition', Systems \& Control Letters 37(3), 117-122.

Malekpour, M. \& Torres, W. (2000), Characterization of a flight control computer with rollback recovery, in 'Proc. of the 19th DASC Digital Avionics Systems Conference', Philadelphia, PA, pp. 3.C.4-1-8.

Meyn, S. \& Tweedie, R. L. (1993), Markov Chains and Stochastic Stability, SpringerVerlag, New York, NY.

Michel, A. N. \& Porter, D. W. (1972), 'On practical stability and finite time stability of discontinuous systems', IEEE Transactions on Circuit Theory 19(2), 123-129.

Mitra, R., Tarn, T. \& Dai, L. (2001), Stability results for switched linear systems, in 'Proc. of the 2001 American Control Conference', Arlington, VA, pp. 1884-1889.

Molchanov, A. P. \& Pyatnitskiy, Y. S. (1989), 'Criteria of asymptotic stability of differential and difference inclusions encoutered in control theory', Systems $\&$ Control Letters 13(1), 59-64. 
Morozan, T. (1976), On the stability of stochastic discrete systems, in 'Control theory and Topics in Functional Analysis: Lectures Presented at an International Seminar Course at Trieste from 11 Sept. to 29 Nov. 1974', International Atomic Energy Agency, Vienna, pp. 225-254.

Nummelin, E. (1984), General Irreducible Markov Chains and Non-Negative Operators, Cambridge University Press, New York, NY.

Papoulis, A. (1991), Probability, Random Variables, and Stochastic Processes, Third Edition, McGraw-Hill, New York, NY.

Patilkulkarni, S., Herencia-Zapana, H., Gray, W. S. \& González, O. R. (2004), On the stability of jump-linear systems driven by finite-state machines with Markovian inputs, in 'Proc. of the 2004 American Control Conference', Boston, MA, pp. 2534-2539.

Rugh, W. J. (1996), Linear System Theory, Second Edition, Prentice Hall, Upper Saddle River, NJ.

Shiryaev, A. N. (1995), Probability, Second Edition, Springer, New York, NY.

Sontag, E. (1981), 'Nonlinear regulation: the piecewise linear approach', IEEE Trans. Automat. Contr. AC-26(4), 346-358.

Srichander, R. \& Walker, B. K. (1993), 'Stochastic stability analysis for continuoustime fault tolerant control systems', Inter. J. of Control 57(2), 433-452.

Tejada, A. (2002), Analysis of error recovery effects on digital flight control systems, Master's thesis, Old Dominion University. 
Tejada, A., González, O. R. \& Gray, W. S. (2007), 'Stability of digital control systems implemented in error-recoverable computers', International Journal of Control . Under review.

Torrisi, F. D. \& Bemporad, A. (2004), 'HYSDEL-a tool for generating computational hybrid models for analysis and synthesis problems', IEEE Trans. Contr. Syst. Technol. 12(2), 235-249.

Xu, X. \& Antsaklis, P. J. (2003), Practical stabilization of integrator switched systems, in 'Proc. of the 2003 American Control Conference', Denver, CO, pp. 27672772 .

Ye, H. \& Michel, A. N. (1998), 'Stability theory for hybrid dynamical systems', IEEE Trans. Automat. Contr. 43(4), 461-474.

Zhai, G. \& Michel, A. N. (2002), 'On practical stability of switched systems', International Journal of Hybrid Systems 2(1), 141-153.

Zhang, H., Gray, W. S. \& González, O. R. (2005), Performance analysis and validation of a recoverable flight control system in a simulated neutron environment, in 'Proc. of the 2005 Guidance, Navigation and Control Conference', San Francisco, CA. Paper 2005-6430. 


\section{APPENDIX A}

\section{SOME ELEMENTS FROM MEASURE THEORY}

\section{A.1 BASIC CONVENTIONS AND DEFINITIONS}

Let $\Omega$ and $\Phi$ denote arbitrary sets with elements $\omega$ and $\phi$, respectively, and let their various subsets be denoted by $\{\omega: \cdots\}$ and $\{\phi: \cdots\}$ (or, if needed to add clarity, by $\{\omega \in \Omega: \cdots\}$ and $\{\phi \in \Phi: \cdots\})$.

Definition A.1.1 A measurable space is a pair $(\Omega, \mathscr{F})$, where $\Omega$ is an arbitrary set, and $\mathscr{F}$ is a $\sigma$-algebra of subsets of $\Omega$.

Definition A.1.2 A function $f: \Omega \rightarrow \Omega^{\prime}$ defined between two measurable spaces, $(\Omega, \mathscr{F})$ and $\left(\Omega^{\prime}, \mathscr{F}^{\prime}\right)$, is called an $\mathscr{F} / \mathscr{F}^{\prime}$-measurable function if for every $B \in \mathscr{F}^{\prime}$, the set $\{\omega \in \Omega: f(\omega) \in B\} \in \mathscr{F}$.

Remark A.1.1 Observe the following.

1. Let $\mathscr{B}\left(\mathbb{R}^{n}\right)$ denote the Borel algebra over $\mathbb{R}^{n}$, where $\mathscr{B}\left(\mathbb{R}^{n}\right)=\mathscr{B}(\mathbb{R}) \otimes$ $\mathscr{B}\left(\mathbb{R}^{n-1}\right)$ for any $n>1$, and $\otimes$ represents the direct product. In the sequel, $\mathscr{B}\left(\mathbb{R}^{n}\right) / \mathscr{B}\left(\mathbb{R}^{k}\right)$-measurable functions will be called Borel functions. Measurable (or Borel) functions will be denoted by lowercase letters $f, g$, and $h$.

2. In probabilistic settings, an $\mathscr{F} / \mathscr{B}(\mathbb{R})$-measurable function is also called a random variable. Similarly, an $\mathscr{F} / \mathscr{B}\left(\mathbb{R}^{n}\right)$-measurable function is called a random vector, and an $\mathscr{F} / \mathscr{F}^{\prime}$-measurable function is called an $\mathscr{F} / \mathscr{F}^{\prime}$-measurable random element. 
3. Throughout the dissertation, it is assumed that every random variable, vector, or element and stochastic process is defined over the same underlying probability space $(\Omega, \mathscr{F}, \mathrm{Pr})$.

4. To simplify the notation, the reference to the fields will be dropped if they are evident from the context. Thus, instead of referring to $f$ as an $\mathscr{F} / \mathscr{F}$ 'measurable function' we will call it a 'measurable (or Borel) function'. Like-

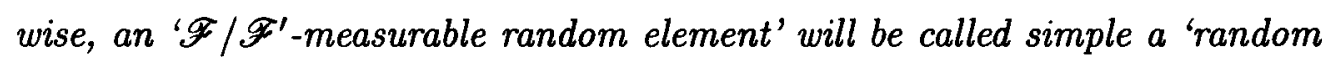
element'.

\section{A.2 SOME PROPERTIES OF $\sigma$-ALGEBRAS}

Let $\mathscr{G}$ be any sub $\sigma$-algebra of $\mathscr{F}$. A random element $x: \Omega \rightarrow \Omega^{\prime}$ is called $\mathscr{G}$ measurable if for every $B \in \mathscr{F}^{\prime}$, the set $\{\omega: \boldsymbol{x}(\omega) \in B\} \in \mathscr{G}$. The smallest $\sigma$-algebra respect to which $\boldsymbol{x}$ is measurable is denoted by $\sigma(\boldsymbol{x})$. The following subsections introduce the properties of $\sigma(\boldsymbol{x})$.

\section{A.2.1 Structure}

Theorem A.2.1 (Theorem 20.1, Billingsley 1995) Let $\boldsymbol{x}=\left(\boldsymbol{x}_{1}, \ldots, \boldsymbol{x}_{n}\right)$ be a vector of random variables, $\boldsymbol{x}_{\boldsymbol{i}}$.

(i) The $\sigma$-algebra $\sigma(\boldsymbol{x})=\sigma\left(\boldsymbol{x}_{1}, \ldots, \boldsymbol{x}_{n}\right)$ consists exactly of the sets $\{\omega \in \Omega$ : $\boldsymbol{x}(\omega) \in B\}$ for $B \in \mathscr{B}\left(\mathbb{R}^{n}\right)$.

(ii) The random variable $\boldsymbol{y}$ is $\sigma(\boldsymbol{x})$-measurable if and only if there exists a measurable function $f: \mathbb{R}^{n} \rightarrow \mathbb{R}$ such that $\boldsymbol{y}(\omega)=f\left(\boldsymbol{x}_{1}(\omega), \ldots, \boldsymbol{x}_{n}(\omega)\right)$ for all $\omega \in \Omega$. 
The previous theorem can be generalized as follows.

Theorem A.2.2 Let $\boldsymbol{x}: \Omega \rightarrow \Omega^{\prime}$ be a random element. The $\sigma$-algebra, $\sigma(\boldsymbol{x})$, consists exactly of the sets $\omega: \boldsymbol{x}(\omega \in H)$ for $H \in \mathscr{F}^{\prime}$.

\section{A.2.2 Combination}

Definition A.2.1 Let $\mathcal{C}$ and $\mathcal{D}$ be two $\sigma$-algebras of subsets of $\Omega$. The binary operation $\AA$ between $\mathcal{C}$ and $\mathcal{D}$ is defined as

$$
\mathcal{C} \oplus \mathcal{D} \triangleq \sigma(\mathcal{C} \cap \mathcal{D})
$$

where $\mathcal{C} \cap \mathcal{D}=\{C \cap D: C \in \mathcal{C}, D \in \mathcal{D}\}$ and $\sigma(\mathcal{C} \cap \mathcal{D})$ denotes the smallest $\sigma$-algebra generated by the sets in $\mathcal{C} \cap \mathcal{D}$.

It follows readily from the definition that $\AA$ is commutative and associative. Moreover, $\mathcal{C} \oplus \mathcal{D}$ can also be defined in terms of unions of sets from $\mathcal{C}$ and $\mathcal{D}$ as shown in the next lemma.

Lemma A.2.1 Let $\mathcal{C}$ and $\mathcal{D}$ be two $\sigma$-algebras of subsets of $\Omega$. Then $\mathcal{C} \oplus \mathcal{D}=\sigma(\mathcal{C} \cup \mathcal{D})$, where $\mathcal{C} \cup \mathcal{D}=\{C \cup D: C \in \mathcal{C}, D \in \mathcal{D}\}$

Proof: Observe that for every $C \in \mathcal{C}$ and $D \in \mathcal{D}$ it follows that $\bar{C} \cap \bar{D} \in \mathcal{C} \oplus \mathcal{D}$. Since $\mathcal{C} \oplus \mathcal{D}$ is a $\sigma$-algebra, then $\overline{\bar{C} \cap \bar{D}}=C \cup D \in \mathcal{C} \oplus \mathcal{D}$. This shows that $\sigma(\mathcal{C} \cup \mathcal{D}) \subset$ $\mathcal{C} \oplus \mathcal{D}$. Conversely, observe from their definitions that $\mathcal{C} \cap \mathcal{D} \subset \mathcal{C} \cup \mathcal{D}$. Consequently, $\mathcal{C} \oplus \mathcal{D}=\sigma(\mathcal{C} \cap \mathcal{D}) \subset \sigma(\mathcal{C} \cup \mathcal{D})$. Thus, $\mathcal{C} \oplus \mathcal{D}=\sigma(\mathcal{C} \cup \mathcal{D})$

The $\oplus$ operation can be used to combine $\sigma$-algebras induced by random variables as described below. 
Lemma A.2.2 For any two random variables $\boldsymbol{x}_{1}, \boldsymbol{x}_{2}, \sigma\left(\boldsymbol{x}_{1}\right) \oplus \sigma\left(\boldsymbol{x}_{2}\right)=\sigma\left(\boldsymbol{x}_{1}, \boldsymbol{x}_{2}\right)$.

Proof: Let $H \in \sigma\left(\boldsymbol{x}_{1}\right) \circledast \sigma\left(\boldsymbol{x}_{2}\right)$. Then $H=H_{1} \cap H_{2}$, where $H_{1} \in \sigma\left(\boldsymbol{x}_{1}\right)$ and $H_{2} \in \sigma\left(\boldsymbol{x}_{2}\right)$. That is, $H_{1}=\left\{\omega: \boldsymbol{x}_{1}(\omega) \in B_{1}\right\}$ and $H_{2}=\left\{\omega: \boldsymbol{x}_{2}(\omega) \in B_{2}\right\}$ for some $B_{1}, B_{2} \in \mathscr{B}(\mathbb{R})$. Thus, it follows that

$$
\begin{aligned}
H & =\left\{\omega: x_{1}(\omega) \in B_{1}\right\} \cap\left\{\omega: x_{2}(\omega) \in B_{2}\right\} \\
& =\left\{\omega:\left(x_{1}(\omega), x_{2}(\omega)\right) \in B_{1} \times B_{2}\right\} \in \sigma\left(x_{1}, x_{2}\right),
\end{aligned}
$$

since $B_{1} \times B_{2} \in \mathscr{B}\left(\mathbb{R}^{2}\right)$. But $H$ is arbitrary, so $\sigma\left(\boldsymbol{x}_{1}\right) \oplus \sigma\left(\boldsymbol{x}_{2}\right) \subseteq \sigma\left(\boldsymbol{x}_{1}, \boldsymbol{x}_{2}\right)$.

To show the converse argument, define $\mathcal{G} \triangleq\left\{H \in \mathscr{B}\left(\mathbb{R}^{2}\right):\left\{\omega:\left(x_{1}(\omega), x_{2}(\omega)\right) \in\right.\right.$ $\left.H\} \subset \sigma\left(x_{1}\right) \oplus \sigma\left(x_{2}\right)\right\}$ and observe that $\mathcal{G}$ is itself a $\sigma$-algebra. It is clear from its definition that $\mathcal{G} \subset \mathscr{B}\left(\mathbb{R}^{2}\right)$. Moreover, observe that for every $B_{1}, B_{2} \in \mathscr{B}\left(\mathbb{R}^{1}\right)$ it follows that $B_{1} \times B_{2} \in \mathcal{G}$, since $\left\{\omega:\left(x_{1}(\omega), x_{2}(\omega)\right) \in B_{1} \times B_{2}\right\}=x_{1}^{-1}\left(B_{1}\right) \cap x_{2}^{-1}\left(B_{2}\right) \in$ $\sigma\left(x_{1}\right) 丹 \sigma\left(x_{2}\right)$. Now, let $\sigma\left(B_{1} \times B_{2}\right)$ denote the smallest $\sigma$-algebra generated from sets of the form $B_{1} \times B_{2}$ and observe that $\mathscr{B}\left(\mathbb{R}^{2}\right)=\sigma\left(B_{1} \times B_{2}\right) \subset \sigma(\mathcal{G})=\mathcal{G} \subset \mathscr{B}\left(\mathbb{R}^{2}\right)$. That is, $\mathcal{G}=\mathscr{B}\left(\mathbb{R}^{2}\right)$. This in turn implies that $\left.\left\{\omega:\left(\boldsymbol{x}_{1}(\omega), x_{2}(\omega)\right) \in H\right\} \subset \sigma\left(\boldsymbol{x}_{1}\right) \oplus \sigma\left(\boldsymbol{x}_{2}\right)\right\}$ for every $H \in \mathscr{B}\left(\mathbb{R}^{2}\right)$. This fact and Theorem A.2.1 show that $\sigma\left(x_{1}, x_{2}\right) \subset \sigma\left(x_{1}\right) \circledast$ $\sigma\left(x_{2}\right)$, completing the proof.

Remark A.2.1 Let $\boldsymbol{x}_{1}, \boldsymbol{x}_{2}$, and $\boldsymbol{x}_{3}$ be random variables, and let $\boldsymbol{y}_{1}=\left(\boldsymbol{y}_{11}, \ldots, \boldsymbol{y}_{1 n}\right)$ and $\boldsymbol{y}_{2}=\left(\boldsymbol{y}_{21}, \ldots, \boldsymbol{y}_{2 n}\right)$ be vectors of random variables.

(i) Observe from Theorem A.2.1, the definition of $\mathrm{A}$, and Lemma A.2.2 that $\sigma\left(\boldsymbol{x}_{1}, \boldsymbol{x}_{2}, \boldsymbol{x}_{3}\right)=\sigma\left(\boldsymbol{x}_{1}\right) \oplus \sigma\left(\boldsymbol{x}_{2}, \boldsymbol{x}_{3}\right)=\sigma\left(\boldsymbol{x}_{1}, \boldsymbol{x}_{2}\right) \oplus \sigma\left(\boldsymbol{x}_{2}, \boldsymbol{x}_{3}\right)$.

(ii) A similar derivation shows that $\sigma\left(\boldsymbol{y}_{1}, \boldsymbol{y}_{2}\right)=\sigma\left(\boldsymbol{y}_{11}, \ldots, \boldsymbol{y}_{1 n}, \boldsymbol{y}_{21}, \ldots, \boldsymbol{y}_{2 n}\right)=$ $\sigma\left(\boldsymbol{y}_{1}\right) \oplus \sigma\left(\boldsymbol{y}_{2}\right)$. 
Both arguments can be extended inductively to any finite number of random variables or random vectors. This remark plays an important role in the following collection of results.

\section{A.2.3 Simplification of $\sigma$-Algebras}

Theorem A.2.3 Let $\boldsymbol{x}=\left(x_{1}, \ldots, \boldsymbol{x}_{n}\right)$ be a vector of random variables, and let $f$ : $\mathbb{R}^{n} \rightarrow \mathbb{R}$ be a measurable function. If $\boldsymbol{y}=f(\boldsymbol{x})$ then $\sigma(\boldsymbol{y}, \boldsymbol{x})=\sigma(\boldsymbol{x})$.

Proof: Observe from Theorem A.2.1 that $\sigma(\boldsymbol{y})$ is composed of exactly all the sets of the form $\{\omega: \boldsymbol{y}(\omega) \in B\}, B \in \mathscr{B}\left(\mathbb{R}^{1}\right)$. Moreover, for every $B \in \mathscr{B}\left(\mathbb{R}^{1}\right)$ it follows that $\{\omega: \boldsymbol{y}(\omega) \in B\}=\left\{\omega: \boldsymbol{x}(\omega) \in f^{-1}(B)\right\}$. Thus, $\sigma(\boldsymbol{y}) \subset \sigma(\boldsymbol{x})$ and $\sigma(\boldsymbol{y}) \cup \sigma(\boldsymbol{x})=\sigma(\boldsymbol{x})$. Finally, note from Lemmas A.2.1 and A.2.2 that $\sigma(\boldsymbol{y}, \boldsymbol{x})=$ $\sigma(\boldsymbol{y}) \oplus \sigma(\boldsymbol{x})=\sigma(\sigma(\boldsymbol{y}) \cup \sigma(\boldsymbol{x}))=\sigma(\sigma(\boldsymbol{x}))=\sigma(\boldsymbol{x})$, which completes the proof.

This result can be extended to the case when $\boldsymbol{y}$ is a vector-valued function of $\boldsymbol{x}$ as follows.

Theorem A.2.4 Let $\boldsymbol{x}=\left(\boldsymbol{x}_{1}, \ldots, \boldsymbol{x}_{n}\right)$ be vector of random variables and $F: \mathbb{R}^{n} \rightarrow$ $\mathbb{R}^{m}$ be a Borel function. If $\boldsymbol{y}=F(\boldsymbol{x})$ then $\sigma(\boldsymbol{y}, \boldsymbol{x})=\sigma(\boldsymbol{x})$.

Proof: First, note that $F=\left(f_{1}, \ldots, f_{m}\right)$, where $f_{i}: \mathbb{R}^{n} \rightarrow \mathbb{R}, i=1, \ldots, m$ are measurable functions. Next, observe that $\boldsymbol{y}=\left(\boldsymbol{y}_{1}, \ldots, \boldsymbol{y}_{m}\right)$, where $\boldsymbol{y}_{i}=f_{i}(\boldsymbol{x})$ and $i=1, \ldots, m$. Thus, it follows from Theorem A.2.3 that $\sigma\left(\boldsymbol{y}_{i}, \boldsymbol{x}\right)=\sigma(\boldsymbol{x})$ for every $i=1, \ldots, m$. Thus, Remark A.2.1 yields

$$
\begin{aligned}
\sigma(\boldsymbol{x})=\sigma\left(\boldsymbol{y}_{1}\right) \oplus \sigma(\boldsymbol{x}) & =\sigma\left(\boldsymbol{y}_{1}\right) \oplus \sigma\left(\boldsymbol{y}_{1}\right) \oplus \sigma(\boldsymbol{x}) \\
& =\sigma\left(\boldsymbol{y}_{1}\right) \oplus \cdots \oplus \sigma\left(\boldsymbol{y}_{m}\right) \oplus \sigma(\boldsymbol{x}),
\end{aligned}
$$


which in turn implies that $\sigma(\boldsymbol{x})=\sigma(\boldsymbol{y}) \oplus \sigma(\boldsymbol{x})=\sigma(\boldsymbol{y}, \boldsymbol{x})$.

\section{A.3 ON INDEPENDENCE AND EXPECTED VALUES}

\section{A.3.1 Independence}

Definition A.3.1 Let $\mathscr{C}_{i}, i=1, \ldots, n$ be a set of sub $\sigma$-algebras of $\mathscr{F}$. Also, let $\boldsymbol{x}_{i}: \Omega \rightarrow \Omega_{i}$ be $\mathscr{F} / \mathscr{F}_{i}$ random elements for $i=1, \ldots, n$.

(i) The $\sigma$-algebras $\mathscr{C}_{1}, \ldots, \mathscr{C}_{n}$ are independent if $\operatorname{Pr}\left\{B_{1} \cap \cdots \cap B_{n}\right\}=\operatorname{Pr}\left\{B_{1}\right\} \ldots$ $\operatorname{Pr}\left\{B_{n}\right\}$ for all $B_{i} \in \mathscr{C}_{i}, i=1,2, \ldots, n$.

(ii) The random elements $\boldsymbol{x}_{1}, \ldots, \boldsymbol{x}_{n}$ are independent if $\sigma\left(\boldsymbol{x}_{1}\right), \ldots, \sigma\left(\boldsymbol{x}_{n}\right)$ are independent.

(iii) A random variable $\boldsymbol{y}$ and the stochastic process $\mathscr{X}=\left\{\boldsymbol{x}_{k}\right\}$ are independent if for every finite integer $n \geq 1$ and every sequence of integers $0 \leq t_{1}<\cdots<$ $t_{n}<\infty$ it follows that $\sigma(\boldsymbol{y})$ and $\sigma\left(\boldsymbol{x}_{t_{1}}, \ldots, \boldsymbol{x}_{t_{n}}\right)$ are independent.

Remark A.3.1 Let $\mathscr{C}$ and $\mathscr{D}$ be independent sub $\sigma$-algebras of $\mathscr{F}$. Clearly, if $\boldsymbol{x}$ and $\boldsymbol{y}$ are, respectively, $\mathscr{C}$-measurable and $\mathscr{D}$-measurable random elements, then $\boldsymbol{x}$ and $\boldsymbol{y}$ are independent.

\section{A.3.2 Expected Values}

Definition A.3.2 Let $\boldsymbol{\xi}$ be an integrable random variable defined on over $(\Omega, \mathscr{F}, \operatorname{Pr})$.

(i) The expected value of $\boldsymbol{\xi}, \mathbf{E}\{\boldsymbol{\xi}\}$, is the (extended) real number given by $\mathbf{E}\{\boldsymbol{\xi}\}=$ $\int_{\Omega} \boldsymbol{\xi} \mathrm{dP}$.

(ii) Let $\mathscr{G} \subset \mathscr{F}$ be a $\sigma$-algebra. The conditional expectation of $\boldsymbol{\xi}$ with respect of $\mathscr{G}$, $\mathbf{E}\{\boldsymbol{\xi} \mid \mathcal{G}\}$, is an (extended) random variable such that 
- $\mathbf{E}\{\boldsymbol{\xi} \mid \mathscr{G}\}$ is $\mathscr{G}$-measurable.

- For every $G \in \mathscr{G}$

$$
\int_{G} \boldsymbol{\xi} \mathrm{dP}=\int_{G} \mathbf{E}\{\boldsymbol{\xi} \mid \mathscr{G}\} \mathrm{dP} .
$$

Note that $\mathbf{E}\{\boldsymbol{\xi} \mid \mathscr{G}\}$ is unique up to sets in $\mathscr{G}$ of measure zero.

Lemma A.3.1 The following are standard results from the literature (cf. Shiryaev 1995).

(i) Let $\boldsymbol{x}$ and $\boldsymbol{y}$ be independent random variables. Then $\mathbf{E}\{\boldsymbol{x} \boldsymbol{y}\}=\mathbf{E}\{\boldsymbol{x}\} \mathbf{E}\{\boldsymbol{y}\}$.

(ii) Let $\boldsymbol{x}=\left(\boldsymbol{x}_{1}, \ldots, \boldsymbol{x}_{n}\right)$ be a vector of random variables. If $\boldsymbol{x}$ is independent of a random variable $\boldsymbol{y}$, then so is each $\boldsymbol{x}_{i}$. Furthermore, $\mathbf{E}\{\boldsymbol{x} \boldsymbol{y}\}=\mathbf{E}\{\boldsymbol{x}\} \mathbf{E}\{\boldsymbol{y}\}$.

(iii) Let $\mathscr{F}_{1}$ and $\mathscr{F}_{2}$ be $\sigma$-algebras such that $\mathscr{F}_{1} \subseteq \mathscr{F}_{2}$. If $\boldsymbol{x}$ is an integrable random variable then

$$
\mathrm{E}\left\{\mathrm{E}\left\{\boldsymbol{x} \mid \mathscr{F}_{1}\right\} \mid \mathscr{F}_{2}\right\}=\mathbf{E}\left\{\mathbf{E}\left\{\boldsymbol{x} \mid \mathscr{F}_{2}\right\} \mid \mathscr{F}_{1}\right\}=\mathbf{E}\left\{\boldsymbol{x} \mid \mathscr{F}_{1}\right\}
$$

(iv) For any $\mathscr{F}_{1}$-measurable random element $\boldsymbol{x}, \mathbf{E}\left\{\boldsymbol{x} \mid \mathscr{F}_{1}\right\}=\boldsymbol{x}$.

The following theorem and its corollary is used for proving the results in Chapter IV.

Theorem A.3.1 Let $\boldsymbol{x}$ be a vector of random variables, and let $\boldsymbol{y}$ and $\boldsymbol{z}$ be two random elements such that $\boldsymbol{z}$ is independent of $(\boldsymbol{x}, \boldsymbol{y})$, i.e., $\sigma(\boldsymbol{z})$ is independent from $\sigma(\boldsymbol{x}), \sigma(\boldsymbol{y})$, and $\sigma(\boldsymbol{x}, \boldsymbol{y})$. Then it follows that $\mathbf{E}\{\boldsymbol{x} \mid \boldsymbol{y} \boldsymbol{z}\}=\mathbf{E}\{\boldsymbol{x} \mid \boldsymbol{y}\}$.

Proof: First, note from the definition that $\int_{G} \mathbf{E}\{\boldsymbol{x} \mid \boldsymbol{y} \boldsymbol{z}\} \mathrm{dP}=\int_{G} \boldsymbol{x} \mathrm{dP}$ for every $G \in$ $\sigma(\boldsymbol{y}, \boldsymbol{z})$. Next, recall that $\sigma(\boldsymbol{y}, \boldsymbol{z})=\sigma(\boldsymbol{y}) \oplus \sigma(\boldsymbol{z})$, so $G=G_{1} \cap G_{2}$ for some $G_{1} \in \sigma(\boldsymbol{y})$ and $G_{2} \in \sigma(z)$. Thus,

$$
\int_{G} x \mathrm{dP}=\int_{\Omega} x 1_{\{G\}} \mathrm{dP}=\mathbf{E}\left\{x 1_{\{G\}}\right\}=\mathbf{E}\left\{x 1_{\left\{G_{1}\right\}} \mathbf{1}_{\left\{G_{2}\right\}}\right\}
$$


where $\mathbf{1}_{\{\cdot\}}$ denotes the Dirac function. Note that $\boldsymbol{x} \mathbf{1}_{\left\{G_{1}\right\}}$ and $\mathbf{1}_{\left\{G_{2}\right\}}$ are, respectively, $\sigma(\boldsymbol{x}, \boldsymbol{y})$-measurable and $\sigma(\boldsymbol{z})$-measurable functions. Thus, the hypothesis and Remark A.3.1 imply that $x 1_{\left\{G_{1}\right\}}$ and $\mathbf{1}_{\left\{G_{2}\right\}}$ are independent, which in turn gives that

$$
\int_{G} \mathbf{E}\{\boldsymbol{x} \mid \boldsymbol{y} z\} \mathrm{dP}=\int_{G} \boldsymbol{x} \mathrm{dP}=\mathbf{E}\left\{\boldsymbol{x} \mathbf{1}_{\left\{G_{1}\right\}}\right\} \mathbf{E}\left\{\mathbf{1}_{\left\{G_{2}\right\}}\right\} .
$$

Next, observe that $\int_{G_{1}} E\{x \mid y\} \mathrm{dP}=\int_{G_{1}} \boldsymbol{x} \mathrm{dP}$ for every $G_{1} \in \sigma(\boldsymbol{y})$. This last expression can be rewritten as $\mathbf{E}\left\{\mathbf{E}\{\boldsymbol{x} \mid \boldsymbol{y}\} \mathbf{1}_{G_{1}}\right\}=\mathbf{E}\left\{\boldsymbol{x} \mathbf{1}_{G_{1}}\right\}$. Clearly, $\mathbf{E}\{\boldsymbol{x} \mid \boldsymbol{y}\} \mathbf{1}_{G_{1}}$ is $\sigma(\boldsymbol{y})$ measurable. Thus in (103)

$$
\begin{aligned}
\int_{G} \mathbf{E}\{\boldsymbol{x} \mid \boldsymbol{y z}\} \mathrm{dP} & =\mathbf{E}\left\{\mathbf{E}\{\boldsymbol{x} \mid \boldsymbol{y}\} \mathbf{1}_{\left\{G_{1}\right\}}\right\} \mathbf{E}\left\{\mathbf{1}_{\left\{G_{2}\right\}}\right\} \\
& =\mathbf{E}\left\{\mathbf{E}\{\boldsymbol{x} \mid \boldsymbol{y}\} \mathbf{1}_{\left\{G_{1}\right\}} \mathbf{1}_{\left\{G_{2}\right\}}\right\} \\
& =\mathbf{E}\left\{\mathbf{E}\{\boldsymbol{x} \mid \boldsymbol{y}\} \mathbf{1}_{\{G\}}\right\} \\
& =\int_{G} \mathbf{E}\{\boldsymbol{x} \mid \boldsymbol{y}\} \mathrm{dP}
\end{aligned}
$$

The conclusion above holds for every $G \in \sigma(\boldsymbol{y}, \boldsymbol{z})$, which proves that $\mathbf{E}\{\boldsymbol{x} \mid \boldsymbol{y}\}$ is a version $^{2}$ of $\mathbf{E}\{\boldsymbol{x} \mid \boldsymbol{y} \boldsymbol{z}\}$, i.e., $\mathbf{E}\{\boldsymbol{x} \mid \boldsymbol{y} \boldsymbol{z}\}=\mathbf{E}\{\boldsymbol{x} \mid \boldsymbol{y}\}$

Corollary A.3.1 Let $N(k)$ be a discrete-time Markov chain in $\mathbb{R}^{m}$ and $\boldsymbol{x}_{0}$ a second order random vector. If $\boldsymbol{x}_{0}$ is independent from $\boldsymbol{N}(k)$ then $\mathbf{E}\{\boldsymbol{N}(k+$ 1) $\left.\mid \boldsymbol{N}(k), \ldots, \boldsymbol{N}(0), x_{0}\right\}=\mathbf{E}\{\boldsymbol{N}(k+1) \mid \boldsymbol{N}(k), \ldots, N(0)\}$.

Proof: Let $\boldsymbol{x}=\boldsymbol{N}(k+1), \boldsymbol{y}=(\boldsymbol{N}(k), \ldots, \boldsymbol{N}(0))$, and $\boldsymbol{z}=\boldsymbol{x}_{0}$. By hypothesis, $\boldsymbol{x}_{0}$ is independent from $(\boldsymbol{N}(k+1), \boldsymbol{N}(k), \ldots, \boldsymbol{N}(0))=(\boldsymbol{x}, \boldsymbol{y})$. Thus, $\boldsymbol{z}$ is independent from $(\boldsymbol{x}, \boldsymbol{y})$ and the result follows from Theorem A.3.1.

\footnotetext{
${ }^{2} \mathrm{~A}$ random variable, $\boldsymbol{f}$, is called a version of $\mathbf{E}\{\boldsymbol{x} \mid \boldsymbol{y}\}$ if they differ only in sets of Pr-measure zero (p. 445, Billingsley 1995).
} 


\section{APPENDIX B}

\section{PROOF OF THEOREMS IV.2.2 AND IV.2.4}

The proof of Theorem IV.2.1 relies on Theorems IV.2.2 and IV.2.4, which are proven in the following two sections.

\section{B.1 PROOF OF THEOREM IV.2.2}

The proof of Theorem IV.2.2 is presented here. It relies on three facts about system (65), which under the conditions of Theorem IV.2.1 are verified first.

Fact(i): The $\sigma$-algebra generated by the joint process $(x(k), N(k))$ can be simplified as follows:

$$
\sigma(\boldsymbol{x}(k), N(k), \ldots, \boldsymbol{x}(0), N(0))=\sigma(N(k), \ldots, N(0), \boldsymbol{x}(0))
$$

Fact(ii): For any integrable random variable $\boldsymbol{\xi}$,

$$
\mathbf{E}\{\boldsymbol{\xi} \mid \boldsymbol{x}(k), \boldsymbol{N}(k), \ldots, \boldsymbol{x}(0), \boldsymbol{N}(0)\}=\mathbf{E}\{\boldsymbol{\xi} \mid \boldsymbol{N}(k), \ldots, \boldsymbol{N}(0), \boldsymbol{x}(0)\}
$$

Fact(iii): $\mathbf{E}\{\boldsymbol{N}(k+1) \mid \boldsymbol{x}(k), \boldsymbol{N}(k)\}=\mathbf{E}\{\boldsymbol{N}(k+1) \mid \boldsymbol{N}(k)\}$

Fact (i) is at the core of the proof of Theorem IV.2.2. It establishes that all the probabilistic information is contained in $\boldsymbol{x}(0)$ and $\boldsymbol{N}(i), i=0, \ldots, k$. That is, no new information can be obtained by considering the variables $\boldsymbol{x}(i), i=0, \ldots, k$, when $\boldsymbol{x}(0)$ and $\boldsymbol{N}(i), i=0, \ldots, k$, are available. Fact (i) is proven in Subsection B.1.1 (Theorem B.1.1). It makes use of the binary operator $\oplus$ and standard results on the structure of $\sigma$-algebras induced by random variables (see Appendix A). Facts (ii) and 
(iii) are proven in Subsection B.1.2 (Theorems B.1.2 and B.1.3). Finally, the proof of Theorem IV.2.2 is presented in Subsection B.1.3.

\section{B.1.1 Proof of Fact (i)}

Theorem B.1.1 Consider system (65) under the conditions of Theorem IV.2.1. It follows that

$$
\sigma(x(k), N(k), \ldots, x(0), N(0))=\sigma(N(k), \ldots, N(0), x(0))
$$

Proof: Note that $\boldsymbol{x}(k)=F_{k}(\boldsymbol{x}(k-1), \boldsymbol{N}(k-1)), k \geq 1$. It follows from Theorem A.2.4 that $\sigma(x(k), x(k-1), N(k-1))=\sigma(x(k-1), N(k-1))$. Trivially then (104) holds for $k=1$. Now, suppose it holds up to some fixed $k=i-1$. Then

$$
\begin{aligned}
& \sigma(\boldsymbol{x}(i), \boldsymbol{N}(n), \ldots, \boldsymbol{x}(0), \boldsymbol{N}(0)) \\
& =\sigma(\boldsymbol{N}(i)) \oplus \sigma(x(i), \boldsymbol{x}(n-1), \boldsymbol{N}(i-1), \ldots, \boldsymbol{x}(0), \boldsymbol{N}(0)) \\
& =\sigma(\boldsymbol{N}(i)) \oplus \sigma(x(i), x(i-1), \boldsymbol{N}(i-1)) \oplus \sigma(x(i-2), \boldsymbol{N}(i-2), \ldots, \boldsymbol{x}(0), \boldsymbol{N}(0)) \\
& =\sigma(\boldsymbol{N}(i)) \oplus \sigma(x(i-1), \boldsymbol{N}(i-1)) \oplus \sigma(x(i-2), \boldsymbol{N}(i-2), \ldots, \boldsymbol{x}(0), \boldsymbol{N}(0)) \\
& =\sigma(N(i)) \oplus \sigma(x(i-1), N(i-1), \ldots, x(0), N(0)) \\
& =\sigma(N(i)) \oplus \sigma(N(i-1), \ldots, N(0), x(0)) \\
& =\sigma(\boldsymbol{N}(i), \ldots, \boldsymbol{x}(0), \boldsymbol{N}(0))
\end{aligned}
$$

Thus (104) also holds for $k=i$, and therefore by induction for all $k \geq 1$. 


\section{B.1.2 Proofs of Facts (ii) and (iii)}

Theorem B.1.2 Consider system (65) under the conditions of Theorem IV.2.1. Then, for any integrable random variable $\boldsymbol{\xi}$,

$$
\mathrm{E}\{\boldsymbol{\xi} \mid \boldsymbol{x}(k), \boldsymbol{N}(k), \ldots, \boldsymbol{x}(0), \boldsymbol{N}(0)\}=\mathbf{E}\{\boldsymbol{\xi} \mid \boldsymbol{N}(k), \ldots, \boldsymbol{N}(0), \boldsymbol{x}(0)\}
$$

Proof: Observe that the integrability of $\boldsymbol{\xi}$ ensures $\mathbf{E}\{\boldsymbol{\xi} \mid \boldsymbol{x}(k), \boldsymbol{N}(k), \ldots, \boldsymbol{x}(0), \boldsymbol{N}(0)\}$ is well defined and

$$
\int_{B} \mathbf{E}\{\boldsymbol{\xi} \mid \boldsymbol{x}(k), \boldsymbol{N}(k), \ldots, \boldsymbol{x}(0), N(0)\} \mathrm{dP}=\int_{B} \boldsymbol{\xi} \mathrm{dP}
$$

for every $B \in \sigma(x(k), N(k), \ldots, x(0), N(0))$. But Theorem B.1.1 shows that $\sigma(x(k), N(k), \ldots, x(0), N(0))=\sigma(\boldsymbol{N}(k), \ldots, N(0), x(0))$. Thus, for every such $B$

$$
\int_{B} \boldsymbol{\xi} \mathrm{dP}=\int_{B} \mathbf{E}\{\boldsymbol{\xi} \mid \boldsymbol{N}(k), \ldots, \boldsymbol{N}(0), \boldsymbol{x}(0)\} \mathrm{dP},
$$

and the result follows.

Theorem B.1.3 Consider system (65) under the conditions of Theorem IV.2.1. Then,

$$
\mathbf{E}\{\boldsymbol{N}(k+1) \mid \boldsymbol{x}(k), \boldsymbol{N}(k)\}=\mathbf{E}\{\boldsymbol{N}(k+1) \mid \boldsymbol{N}(k)\}
$$

Proof: First, note that $\sigma(x(k), N(k))$ is comprised of sets of the form $\{\omega$ : $(\boldsymbol{x}(k, \omega), \boldsymbol{N}(k, \omega)) \in H\}, H \in \mathscr{B}\left(\mathbb{R}^{n}\right) \otimes \mathscr{B}\left(\mathbb{R}^{m}\right)$. Fix any $H \in \mathscr{B}\left(\mathbb{R}^{n}\right) \otimes \mathscr{B}\left(\mathbb{R}^{m}\right)$ and observe that

$$
\{\omega:(\boldsymbol{x}(k, \omega), \boldsymbol{N}(k, \omega)) \in H\}=\left\{\omega: \boldsymbol{x}(k, \omega) \in H_{1}\right\} \cap\left\{\omega: \boldsymbol{N}(k, \omega) \in H_{2}\right\}
$$


for some $H_{1} \in \mathscr{B}\left(\mathbb{R}^{n}\right)$ and $H_{2} \in \mathscr{B}\left(\mathbb{R}^{m}\right)$. Next, note that (65) yields

$$
\begin{aligned}
x(k) & =F_{k-1}(x(k-1), N(k-1)) \\
& =F_{k-1}\left(F_{k-2}(x(k-2), N(k-2)), N(k-1)\right) \\
& =F_{k-1}\left(F_{k-2}\left(F_{k-3}(x(k-3), N(k-3), N(k-2)), N(k-1)\right)\right. \\
& =F_{k-1}\left(F_{k-2}\left(\ldots\left(F_{0}(x(0), N(0)), N(1)\right) \ldots, N(k-2)\right), N(k-1)\right) \\
& \vdots \\
& =G(N(k-1), \ldots, N(0), x(0)),
\end{aligned}
$$

where $G$ is the indicated composition of the functions $F_{0}, \ldots, F_{k-1}$. Thus, (105) can be expressed as

$$
\begin{aligned}
\{\omega: & (\boldsymbol{x}(k, \omega), \boldsymbol{N}(k, \omega)) \in H\} \\
& =\left\{\omega:(\boldsymbol{N}(k-1, \omega), \ldots, \boldsymbol{N}(0, \omega), \boldsymbol{x}(0, \omega)) \in G^{-1}\left(H_{1}\right)\right\} \cap\left\{\omega: \boldsymbol{N}(k, \omega) \in H_{2}\right\} \\
& =\left\{\omega:(\boldsymbol{N}(k, \omega), \ldots, \boldsymbol{N}(0, \omega), \boldsymbol{x}(0, \omega)) \in H_{2} \times G^{-1}\left(H_{1}\right)\right\} .
\end{aligned}
$$

Since $G$ is measurable, it follows that

$$
H_{2} \times G^{-1}\left(H_{1}\right) \in \underbrace{\mathscr{B}\left(\mathbb{R}^{m}\right) \otimes \cdots \otimes \mathscr{B}\left(\mathbb{R}^{m}\right)}_{\mathrm{k}+1 \text { copies }} \otimes \mathscr{B}\left(\mathbb{R}^{n}\right),
$$

which in turn implies that $\{\omega:(\boldsymbol{x}(k, \omega), \boldsymbol{N}(k, \omega)) \in H\} \in \sigma(\boldsymbol{N}(k), \ldots, \boldsymbol{N}(0), \boldsymbol{x}(0))$. But since $H$ is arbitrary, it follows that $\sigma(x(k), \boldsymbol{N}(k)) \subseteq \sigma(\boldsymbol{N}(k), \ldots, \boldsymbol{N}(0), \boldsymbol{x}(0))$. This conclusion, Lemma A.3.1, Corollary A.3.1, and the Markov property of $\boldsymbol{N}(k)$ yield

$$
\begin{aligned}
\mathbf{E}\{\boldsymbol{N}(k+1) \mid \boldsymbol{x}(k), \boldsymbol{N}(k)\} & =\mathbf{E}\{\mathbf{E}\{\boldsymbol{N}(k+1) \mid \boldsymbol{N}(k), \ldots, \boldsymbol{N}(0), \boldsymbol{x}(0)\} \mid \boldsymbol{x}(k), \boldsymbol{N}(k)\} \\
& =\mathbf{E}\{\mathbf{E}\{\boldsymbol{N}(k+1) \mid \boldsymbol{N}(k), \ldots, \boldsymbol{N}(0)\} \mid \boldsymbol{x}(k), \boldsymbol{N}(k)\} \\
& =\mathbf{E}\{\mathbf{E}\{\boldsymbol{N}(k+1) \mid \boldsymbol{N}(k)\} \mid \boldsymbol{x}(k), \boldsymbol{N}(k)\} .
\end{aligned}
$$


Finally, note that $\mathbf{E}\{\boldsymbol{N}(k+1) \mid \boldsymbol{N}(k)\}$ is $\sigma(\boldsymbol{N}(k))$-measurable. Also note that $\sigma(\boldsymbol{N}(k)) \subseteq \sigma(x(k), \boldsymbol{N}(k))$, so $\mathbf{E}\{\boldsymbol{N}(k+1) \mid \boldsymbol{N}(k)\}$ is also $\sigma(\boldsymbol{x}(k), \boldsymbol{N}(k))$-measurable. Thus, Lemma A.3.1 yields $\mathbf{E}\{\mathbf{E}\{\boldsymbol{N}(k+1) \mid \boldsymbol{N}(k)\} \mid x(k), \boldsymbol{N}(k)\}=\mathbf{E}\{\boldsymbol{N}(k+1) \mid \boldsymbol{N}(k)\}$, and the result follows.

\section{B.1.3 Proof of Theorem IV.2.2}

Proof : First, observe $f(N(k+1))$ is an integrable random variable for any bounded Borel function $f: \mathbb{R}^{m} \rightarrow \mathbb{R}$. Thus, Theorem B.1.2 yields

$$
\begin{aligned}
\mathbf{E}\{f(\boldsymbol{N}(k+1)) \mid \boldsymbol{x}(k), \boldsymbol{N}(k), \ldots, & \boldsymbol{x}(0), \boldsymbol{N}(0)\} \\
= & \mathbf{E}\{f(\boldsymbol{N}(k+1)) \mid \boldsymbol{N}(k), \ldots, \boldsymbol{N}(0), \boldsymbol{x}(0)\}
\end{aligned}
$$

Next, note that $f(N(k+1))$ is $\sigma(N(k+1))$-measurable (Theorem A.2.1), so $\sigma(f(\boldsymbol{N}(k+1)), \boldsymbol{N}(k), \ldots, \boldsymbol{N}(0)) \subseteq \sigma(\boldsymbol{N}(k+1), \ldots, \boldsymbol{N}(0))$. Furthermore, this and the hypothesis of Theorem IV.2.2 imply that $x(0)$ is independent of $(f(N) k+$ 1)), $\boldsymbol{N}(k), \ldots, \boldsymbol{N}(0))$. Hence, (106), Theorem A.3.1, and the Markov nature of $\boldsymbol{N}(k)$ yield

$$
\begin{aligned}
\mathbf{E}\{f(\boldsymbol{N}(k+1)) \mid \boldsymbol{x}(k), \boldsymbol{N}(k), \ldots, \boldsymbol{x}(0), \boldsymbol{N}(0)\} & =\mathbf{E}\{f(\boldsymbol{N}(k+1)) \mid \boldsymbol{N}(k), \ldots, \boldsymbol{N}(0)\} \\
& =\mathbf{E}\{f(\boldsymbol{N}(k+1)) \mid \boldsymbol{N}(k)\}
\end{aligned}
$$

From Theorem B.1.3, $\sigma(\boldsymbol{x}(k), \boldsymbol{N}(k)) \subseteq \sigma\left(\boldsymbol{N}(k), \ldots, \boldsymbol{N}(0), \boldsymbol{x}_{0}\right)$. Thus, the argument in the proof of Theorem B.1.3 yields $\mathrm{E}\{f(\boldsymbol{N}(k+1)) \mid \boldsymbol{x}(k), \boldsymbol{N}(k)\}=\mathbf{E}\{f(\boldsymbol{N}(k+$ 1))|N(k)\}, which together with (107) confirm identity (66). 


\section{B.2 PROOF OF THEOREM IV.2.4}

Proof: Observe that $\mathcal{H}$ is a linear space since any linear combination of elements from $\mathcal{H}$ yields a bounded Borel function that satisfies (67), as a consequence of the linearity of the conditional expectation.

Now, to prove (i) in Theorem IV.2.3, suppose $f(\phi)=a$ for all $\phi \in \Phi$ and some $a \in \mathbb{R}$. Note that $f(x(k), N(k), N(k+1))$ is a constant random variable. Also, recall that for any $\sigma$-algebra $\mathscr{G}, \mathrm{E}\{f \mid \mathscr{G}\}=a$ (a.e.) (p. 215, Shiryaev 1995), which in turn implies that $f$ satisfies (67). Since this is true for any $a \in \mathbb{R}$, then $\mathcal{H}$ contains all the constant functions.

To prove (ii), suppose that $\left\{f_{n}\right\} \in \mathcal{H}$ converges uniformly to $f$, and let $L_{n}$ be finite constants such that $\left|f_{n}(\phi)\right|<L_{n}$ for all $\phi \in \Phi$. Next, fix $\epsilon>0$ and observe from the hypothesis that there exists $N(\epsilon)$ such that $\left|f_{n}(\phi)-f(\phi)\right|<\epsilon$ for all $n \geq N(\epsilon)$ and for all $\phi \in \Phi$. In particular, $\left|f_{N(\epsilon)}(\phi)-f(\phi)\right|<\epsilon$ implies that $|f(\phi)|<L_{N(\epsilon)}+\epsilon$ for every $\phi \in \Phi$. Note that the uniform convergence of $\left\{f_{n}\right\}$ implies that it also converges pointwise almost everywhere to $f$. This in turn shows $f$ is measurable (Corollary 2.2.4, Friedman 1982). Hence $f$ is a bounded Borel function.

Set $L=L_{N(\epsilon)}+\epsilon$ and observe that $\left|f_{n}(\phi)-f(\phi)\right|<\epsilon, n \geq N(\epsilon)$ implies that $\left|f_{n}(\phi)\right|<L+\epsilon$ for all $\phi \in \Phi$ and all $n \geq N(\epsilon)$. Next, define the function $g: \Phi \rightarrow \mathbb{R}$ as $g(\phi)=\max \left\{L_{1}, \ldots, L_{N(\epsilon)-1}, L+\epsilon\right\}$, for all $\phi \in \Phi$, and let $\boldsymbol{y}_{n}=f_{n}(\boldsymbol{x}(k), \boldsymbol{N}(k), \boldsymbol{N}(k+1))$ and $\boldsymbol{y}=g(\boldsymbol{x}(k), \boldsymbol{N}(k), \boldsymbol{N}(k+1))$. Observe that $\left\{\boldsymbol{y}_{n}\right\}$ converges pointwise to $f(\boldsymbol{x}(k), \boldsymbol{N}(k), \boldsymbol{N}(k+1))$, that $\left|\boldsymbol{y}_{n}\right|<\boldsymbol{y}$, and that $\mathbf{E}\{\boldsymbol{y}\}=\max \left\{L_{1}, \ldots, L_{N(\epsilon)-1}, L+\epsilon\right\}<\infty$. Hence, it follows that for any $\sigma$-algebra $\mathscr{G}, \mathbf{E}\left\{\boldsymbol{y}_{n} \mid \mathscr{G}\right\}$ converges to $\mathbf{E}\{f(\boldsymbol{x}(k), \boldsymbol{N}(k), \boldsymbol{N}(k+1)) \mid \mathscr{G}\}$ (p. 218, Shiryaev 1995). 
Thus,

$$
\begin{aligned}
\lim _{n \rightarrow \infty} \mathbf{E}\left\{\boldsymbol{y}_{n} \mid \boldsymbol{x}(k), \boldsymbol{N}(k), \ldots, \boldsymbol{x}(0), \boldsymbol{N}(0)\right\} & \\
= & \mathbf{E}\{f(\boldsymbol{x}(k), \boldsymbol{N}(k), \boldsymbol{N}(k+1)) \mid \boldsymbol{x}(k), \boldsymbol{N}(k), \ldots, \boldsymbol{x}(0), \boldsymbol{N}(0)\}
\end{aligned}
$$

But $\left\{f_{n}\right\} \in \mathcal{H}$. Thus, (67) implies that

$$
\begin{aligned}
\lim _{n \rightarrow \infty} \mathbf{E}\left\{\boldsymbol{y}_{n} \mid \boldsymbol{x}(k), \boldsymbol{N}(k), \ldots, \boldsymbol{x}(0), \boldsymbol{N}(0)\right\} & =\lim _{n \rightarrow \infty} \mathbf{E}\left\{\boldsymbol{y}_{n} \mid \boldsymbol{x}(k), \boldsymbol{N}(k)\right\} \\
& =\mathbf{E}\{f(\boldsymbol{x}(k), \boldsymbol{N}(k), \boldsymbol{N}(k+1)) \mid \boldsymbol{x}(k), \boldsymbol{N}(k)\}
\end{aligned}
$$

Hence, $f \in \mathcal{H}$, and (ii) follows.

To prove (iii), let $\left\{f_{n}\right\}$ be a monotone sequence of functions in $\mathcal{H}$ such that $0 \leq f_{n} \leq M<\infty$, and note that $\left\{f_{n}\right\}$ converges pointwise to a function $f$, which is in turn a bounded Borel function. Thus, (iii) follows by using the same argument as in (ii).

To verify (iv), consider the set $\mathcal{C}$ composed of all the bounded separable functions $c: \Phi \rightarrow \mathbb{R}$ of the form $c(\phi)=\hat{c}_{1}(\gamma) \hat{c}_{2}(\lambda), \phi=(\gamma, \lambda)$, where $\hat{c}_{1}: \mathbb{R}^{n} \times \mathbb{R}^{m} \rightarrow \mathbb{R}$ and $\hat{c}_{2}: \mathbb{R}^{m} \rightarrow \mathbb{R}$ are bounded Borel functions.

It is necessary to show that if $c(\phi), d(\phi) \in \mathcal{C}$ then $c(\phi) d(\phi) \in \mathcal{C}$, and that $\mathcal{C} \subseteq \mathcal{H}$. Note that $c(\phi) d(\phi)=\left(\hat{c}_{1}(\gamma) \hat{d}_{1}(\gamma)\right)\left(\hat{c}_{2}(\lambda) \hat{d}_{2}(\lambda)\right)$. But $\hat{c}_{1}(\gamma) \hat{d}_{1}(\gamma)$ and $\hat{c}_{2}(\lambda) \hat{d}_{2}(\lambda)$ are real, bounded Borel functions in, respectively, $\mathbb{R}^{n} \times \mathbb{R}^{m}$ and $\mathbb{R}^{m}$. Thus $c(\phi) d(\phi) \in \mathcal{C}$.

Finally, to see that $\mathcal{C} \in \mathcal{H}$, recall from (p. 216, Shiryaev 1995) that if $\boldsymbol{\xi}$ and $\boldsymbol{\eta}$ are random variables such that $\mathbf{E}\{|\boldsymbol{\xi}|\}<\infty, \mathbf{E}\{|\boldsymbol{\xi} \boldsymbol{\eta}|\}<\infty$, and if $\boldsymbol{\eta}$ is $\mathscr{G}$-measurable, then $\mathbf{E}\{\boldsymbol{\xi} \eta \mid \mathscr{G}\}=\boldsymbol{\eta} \mathbf{E}\{\boldsymbol{\xi} \mid \mathscr{G}\}$. Thus, let $c(\phi)=\hat{c}_{1}(\gamma) \hat{c}_{2}(\lambda) \in \mathcal{C}, \phi=(\gamma, \lambda)$, and observe from Theorem IV.2.2 that 


$$
\begin{aligned}
\mathbf{E}\{c((\boldsymbol{x}(k), \boldsymbol{N}(k)), & \boldsymbol{N}(k+1)) \mid \boldsymbol{x}(k), \boldsymbol{N}(k), \ldots, \boldsymbol{x}(0), \boldsymbol{N}(0)\} \\
& =\mathbf{E}\left\{\hat{c}_{1}(\boldsymbol{x}(k), \boldsymbol{N}(k)) \hat{c}_{2}(\boldsymbol{N}(k+1)) \mid \boldsymbol{x}(k), \boldsymbol{N}(k), \ldots, \boldsymbol{x}(0), \boldsymbol{N}(0)\right\} \\
& =\hat{c}_{1}(\boldsymbol{x}(k), \boldsymbol{N}(k)) \mathbf{E}\left\{\hat{c}_{2}(\boldsymbol{N}(k+1)) \mid \boldsymbol{x}(k), \boldsymbol{N}(k), \ldots, \boldsymbol{x}(0), \boldsymbol{N}(0)\right\} \\
& =\hat{c}_{1}(\boldsymbol{x}(k), \boldsymbol{N}(k)) \mathbf{E}\left\{\hat{c}_{2}(\boldsymbol{N}(k+1)) \mid \boldsymbol{x}(k), \boldsymbol{N}(k)\right\} \\
& =\mathbf{E}\left\{\hat{c}_{1}(\boldsymbol{x}(k), \boldsymbol{N}(k)) \hat{c}_{2}(\boldsymbol{N}(k+1)) \mid \boldsymbol{x}(k), \boldsymbol{N}(k)\right\} \\
& =\mathbf{E}\{c((\boldsymbol{x}(k), \boldsymbol{N}(k)), \boldsymbol{N}(k+1)) \mid \boldsymbol{x}(k), \boldsymbol{N}(k)\}
\end{aligned}
$$

Thus, $c$ satisfies (67). Since this is true for every $c \in \mathcal{C}$, then $\mathcal{C} \in \mathcal{H}$ and (iv) follows. This completes the proof. - 


\section{APPENDIX C}

\section{A-EQUIVALENCE}

\section{C.1 INTRODUCTION}

This appendix formalizes the concept of A-equivalence in the sense of Definition 3.2 in (Patilkulkarni et al. 2004). The discussion is based on the concepts developed on Chapter IV, some of which will be stated here without proof.

Definition C.1.1 Two jump linear systems

$$
\boldsymbol{x}(k+1)=A_{\boldsymbol{\theta}(k)} \boldsymbol{x}(k)
$$

and

$$
\tilde{\boldsymbol{x}}(k+1)=\tilde{A}_{\varphi(k)} \tilde{\boldsymbol{x}}(k),
$$

are said to be $\boldsymbol{A}$-equivalent if, for every $k \geq 0, A_{\boldsymbol{\theta}(k)}=\tilde{A}_{\boldsymbol{\varphi}(k)}$.

Note that the processes $\{\theta(k), k \geq 0\}$ and $\{\varphi(k), k \geq 0\}$ does not need to take on values from the same symbol set. Also note that, under A-equivalence, if $x(0)=$ $\tilde{\boldsymbol{x}}(0)=x_{0}$ then $\boldsymbol{x}(k)=\tilde{\boldsymbol{x}}(k)$ for all $k \geq 0$. Thus it is expected that the limit behavior of $\boldsymbol{x}(k)$ and $\tilde{\boldsymbol{x}}(k)$ should also be the same, as show in the following theorem.

Theorem C.1.1 (Theorem 3.3, Patilkulkarni et al. 2004) Let the jump linear systems (108) and (109) be A-equivalent. Then, (108) is MSS if and only if (109) is MSS. 
This theorem was proved in (Patilkulkarni et al. 2004) assuming that $\{\boldsymbol{\theta}(k), k \geq 0\}$ and $\{\varphi(k), k \geq 0\}$ were the output processes of compatible FSM's. In what follows, this theorem will be proven in the context of HJLS's.

\section{C.2 A-EQUIVALENCE OF HJLS's}

A-equivalency can be defined in the context of HJLS's as follows.

Definition C.2.1 Two HJLS's are said to be A-equivalent if their respective jump linear closed-loop systems are A-equivalent.

Consider the following HJLS's

$$
\begin{aligned}
\boldsymbol{x}(k+1) & =A_{\boldsymbol{z}(k)} \boldsymbol{x}(k) \\
\boldsymbol{z}(k+1) & =S_{\boldsymbol{N}(k), \psi(\boldsymbol{x}(k))} \boldsymbol{z}(k) \\
\psi(x) & =\sum_{i=0}^{\ell_{\nu}-1} i \mathbf{1}_{\left\{\boldsymbol{x} \in R_{i}\right\}}, \quad \bigcup_{i \in \mathcal{I}_{\ell_{\nu}}} R_{i}=\mathbb{R}^{n} .
\end{aligned}
$$

and

$$
\begin{aligned}
\tilde{\boldsymbol{x}}(k+1) & =\tilde{A}_{\tilde{z}(k), \boldsymbol{N}(k)} \tilde{x}(k) \\
\tilde{\boldsymbol{z}}(k+1) & =S_{\boldsymbol{N}(k), \psi(\tilde{x}(k))} \tilde{\boldsymbol{z}}(k) \\
\psi(x) & =\sum_{i=0}^{\ell_{\nu}-1} i \mathbf{1}_{\left\{x \in R_{i}\right\}}, \quad \bigcup_{i \in \mathcal{I}_{\ell_{\nu}}} R_{i}=\mathbb{R}^{n} .
\end{aligned}
$$

Note that both HJLS's have the same $A / S$ map. The following lemma is trivial.

Lemma C.2.1 Suppose that $A_{\boldsymbol{z}(k)}=A_{\tilde{\mathbf{z}}(k), \boldsymbol{N}(k)}=A_{i}$, whenever $\boldsymbol{z}(k)=\tilde{\boldsymbol{z}}(k)=$ $e_{i}$. Then, the jump linear systems embedded in the HJLS's (110) and (111) are Aequivalent provided that $\boldsymbol{x}(0)=\tilde{\boldsymbol{x}}(0)=x_{0}$ and $\boldsymbol{z}(0)=\tilde{z}(0)=z_{0}$. 
Note that under the conditions of Lemma C.2.1 both the jump linear systems and the FSM's are A-equivalent. This sets the conditions for the next theorem.

Theorem C.2.1 Consider the HJLS's (110) and (111) under the conditions of Lemma C.2.1 and Assumption IV.3.1. Then, for any fixed $x_{0}$ and $z_{0}$ it follows that $\lim _{k \rightarrow 0} \mathrm{E}\left\{\|\boldsymbol{x}(k)\|^{2}\right\}=0$ if and only if $\lim _{k \rightarrow 0} \mathbf{E}\left\{\|\tilde{\boldsymbol{x}}(k)\|^{2}\right\}=0$.

Proof: The idea behind the proof is to show that $\mu_{k}^{x}=\mu_{k}^{\tilde{x}}$ for every $k \geq 0$. This in turn shows that $\mathbf{E}\left\{\|\boldsymbol{x}(k)\|^{2}\right\}=\mathbf{E}\left\{\|\tilde{\boldsymbol{x}}(k)\|^{2}\right\}$ for every $k \geq 0$, which proves the theorem.

First, observe that under the conditions of Lemma C.2.1 $\mu_{0}^{x}=\mu_{0}^{\bar{x}}=\delta_{x_{0}}$. Next, recall from Chapter IV that $\boldsymbol{y}(k)=\left[\boldsymbol{x}(k)^{\mathrm{T}} \boldsymbol{z}(k)^{\mathrm{T}}\right]^{\mathrm{T}}$ and from Theorem IV.3.2 that

$$
\begin{aligned}
& \mu_{k}^{x}(B)=\mu_{k}^{(y, N)}\left(B \times \Sigma_{s} \times \mathcal{I}_{\ell_{N}}\right)= \\
& \sum_{r_{0}=1}^{\ell_{S}} \sum_{i_{0}=0}^{\ell_{N}-1} \cdots \sum_{r_{k-1}=1}^{\ell_{S}} \sum_{i_{k-1}=0}^{\ell_{N}-1} \mu_{0}^{(z, N)}\left(\left\{\left(e_{r_{0}}, i_{0}\right)\right\}\right) \underset{\left(r_{0}, i_{0}\right),\left(r_{1}, i_{1}\right)}{\mathrm{P}}(\bar{x}) \underset{\left(r_{1}, i_{1}\right),\left(r_{2}, i_{2}\right)}{\mathrm{P}}\left(A_{e_{r_{0}}} \bar{x}\right) \cdots \\
& \underset{\left(r_{k-2}, i_{k-2}\right),\left(r_{k-1}, i_{k-1}\right)}{\mathrm{P}}\left(A_{e_{r_{k-3}}} \cdots A_{e_{r_{0}}} \bar{x}\right) \delta_{A_{e_{r_{k-1}}} \cdots A_{e_{r_{0}}} \bar{x}}(B) .
\end{aligned}
$$

Now, let $\tilde{\boldsymbol{y}}(k)=\left[\tilde{\boldsymbol{x}}(k)^{\mathbf{T}} \tilde{\boldsymbol{z}}(k)^{\mathrm{T}}\right]^{\mathrm{T}}$. Observe that a derivation similar to the one leading to (84) yields

$$
\mathrm{P}_{(\bar{y}, N)}\left(\left(\left[\begin{array}{c}
x \\
e_{r}
\end{array}\right], i\right), B \times C \times D\right)=\sum_{l=0}^{\ell_{N}-1} \mathbf{1}_{B}\left(A_{e_{r}, i} z\right) \mathbf{1}_{C}\left(S_{i, \psi(x)} e_{r}\right) \mathbf{1}_{D}(l) p_{i, l}^{N} .
$$

Thus, it is possible to compute $\mu_{1}^{(\tilde{y}, N)}$ as follows:

$$
\begin{aligned}
\mu_{1}^{(\tilde{y}, N)}(B & \times C \times D) \\
& =\mathrm{P}_{(\tilde{y}, N)} \mu_{0}^{(\tilde{y}, N)}(B \times C \times D) \\
& =\int_{\Sigma_{S} \times \mathcal{I}_{\ell_{N}}} \int_{\mathbb{R}^{n}}^{\ell_{N}-1} \sum_{l=0}^{1} 1_{B}\left(A_{z, i} x\right) \mathbf{1}_{C}\left(S_{i, \psi(x)} z\right) \mathbf{1}_{D}(l) p_{i, l}^{N} \delta_{x_{0}}(d x) \mu_{0}^{(\tilde{z}, N)}(d i \times d z)
\end{aligned}
$$




$$
\begin{aligned}
& =\int_{\Sigma_{S} \times \mathcal{I}_{\ell_{N}}} \sum_{l=0}^{\ell_{N}-1} 1_{B}\left(A_{z, i} x_{0}\right) \mathbf{1}_{C}\left(S_{i, \psi\left(x_{0}\right)} z\right) \mathbf{1}_{D}(l) p_{i, l}^{N} \mu_{0}^{(\bar{z}, N)}(d i \times d z) \\
& =\sum_{r_{0}=1}^{\ell_{S}} \sum_{i_{0}=0}^{\ell_{N}-1} \mu_{0}^{(\bar{z}, N)}\left(\left\{\left(e_{r_{0}}, i_{0}\right)\right\}\right) \mathbf{1}_{B}\left(A_{e_{r_{0}}, i_{0}} x_{0}\right) \sum_{l=0}^{\ell_{N}-1} 1_{C}\left(S_{i_{0}, \psi\left(x_{0}\right)} e_{r_{0}}\right) \mathbf{1}_{D}(l) p_{i_{0} l}^{N} \\
& =\sum_{r_{0}=1}^{\ell_{S}} \sum_{i_{0}=0}^{\ell_{N}-1} \mu_{0}^{(\tilde{z}, N)}\left(\left\{\left(e_{r_{0}}, i_{0}\right)\right\}\right) \delta_{A_{e_{r_{0}}, i_{0}} x_{0}}(B) \underset{\left(r_{0}, i_{0}\right),(C, D)}{\mathrm{P}}\left(x_{0}\right)
\end{aligned}
$$

Note that in the previous expression, $\mu_{0}^{(\bar{z}, N)}=\mu_{0}^{(z, N)}$ due to the conditions of Lemma C.2.1 and Assumption IV.3.1. Also, $\underset{\left(r_{0}, i_{0}\right),(C, D)}{\mathrm{P}}\left(x_{0}\right)$ is defined as in (75). Thus, repeating the procedure leading to Theorem IV.3.2 yields

$$
\begin{aligned}
& \mu_{k}^{(\bar{y}, N)}(B \times C \times D)= \\
& \sum_{r_{0}=1}^{\ell_{S}} \sum_{i_{0}=0}^{\ell_{N}-1} \cdots \sum_{r_{k-1}=1}^{\ell_{S}} \sum_{i_{k-1}=0}^{\ell_{N}-1} \mu_{0}^{(\bar{z}, N)}\left(\left\{\left(e_{r_{0}}, i_{0}\right)\right\}\right) \underset{\left(r_{0}, i_{0}\right),\left(r_{1}, i_{1}\right)}{\mathrm{P}}(\bar{x}) \underset{\left(r_{1}, i_{1}\right),\left(r_{2}, i_{2}\right)}{\mathrm{P}}\left(A_{e_{r_{0}}, i_{0}} \bar{x}\right) \cdots \\
& \underset{\left(r_{k-2}, i_{k-2}\right),\left(r_{k-1}, i_{k-1}\right)}{\mathrm{P}}\left(A _ { e _ { r _ { k - 3 } , i _ { k - 3 } } } \cdots A _ { e _ { r _ { 0 } , i _ { 0 } } \overline { x } ) } \underset { ( r _ { k - 1 } , i _ { k - 1 } ) , ( C , D ) } { \mathrm { P } } \left(A_{e_{r_{k-2}, i_{k-2}}} \cdots A_{\left.e_{r_{0}, i_{0}} \bar{x}\right)}\right.\right. \\
& \delta_{A_{e_{r_{k-1}, i_{k-1}}} \cdots A_{e_{r_{0}, i_{0}} \bar{x}}}(B) .
\end{aligned}
$$

Recall that the jump linear systems in (110) and (111) are A-equivalent. Thus, for any $k \geq 0$ it follows that $A_{r_{k}, i_{k}}=A_{r_{k}}$. Using this equality in the expression above, and replacing $C=\Sigma_{S}$ and $D=\mathcal{I}_{\ell_{N}}$ yields

$$
\begin{aligned}
& \mu_{k}^{\tilde{x}}(B)=\mu_{k}^{(\tilde{y}, N)}\left(B \times \Sigma_{S} \times \mathcal{I}_{\ell_{N}}\right)= \\
& \sum_{r_{0}=1}^{\ell_{S}} \sum_{i_{0}=0}^{\ell_{N}-1} \cdots \sum_{r_{k-1}=1}^{\ell_{S}} \sum_{i_{k-1}=0}^{\ell_{N}-1} \mu_{0}^{(\bar{z}, N)}\left(\left\{\left(e_{r_{0}}, i_{0}\right)\right\}\right) \underset{\left(r_{0}, i_{0}\right),\left(r_{1}, i_{1}\right)}{\mathrm{P}}(\bar{x}) \underset{\left(r_{1}, i_{1}\right),\left(r_{2}, i_{2}\right)}{\mathrm{P}}\left(A_{e_{r_{0}}} \bar{x}\right) \cdots \\
& \underset{\left(r_{k-2}, i_{k-2}\right),\left(r_{k-1}, i_{k-1}\right)}{\mathrm{P}}\left(A_{e_{r_{k-3}}} \cdots A_{e_{r_{0}}} \bar{x}\right) \delta_{A_{e_{r_{k-1}}} \cdots A_{e_{r_{0}}} \bar{x}}(B) .
\end{aligned}
$$


Comparing (112) and (114) clearly shows that $\mu_{k}^{x}(B)=\mu_{k}^{\tilde{x}}(B)$ for every $k \geq 0$ and every $B \in \mathscr{B}\left(\mathbb{R}^{n}\right)$ (except, possibly, on sets of measure zero). Clearly,

$$
\mathbf{E}\left\{\|\boldsymbol{x}(k)\|^{2}\right\}=\int_{\mathbb{R}^{n}}\|x\|^{2} \mu_{k}^{x}(d x)=\int_{\mathbb{R}^{n}}\|x\|^{2} \mu_{k}^{\bar{x}}(d x)=\mathbf{E}\left\{\|\tilde{\boldsymbol{x}}(k)\|^{2}\right\},
$$

which in turn implies that $\lim _{k \rightarrow 0} \mathbf{E}\left\{\|\boldsymbol{x}(k)\|^{2}\right\}=0$ if and only if $\lim _{k \rightarrow 0} \mathbf{E}\left\{\|\tilde{\boldsymbol{x}}(k)\|^{2}\right\}=$ 0.

Corollary C.2.1 Under the conditions of Theorem C.2.1, (110) is MSS if and only if (111) is MSS.

Proof : (Sufficiency) The assertion follows immediately by observing that (110) is MSS when $\lim _{k \rightarrow 0} \mathbf{E}\left\{\|\boldsymbol{x}(k)\|^{2}\right\}=0$ for every initial condition $\left[\begin{array}{ll}x_{0}^{\mathrm{T}} & z_{0}^{\mathrm{T}}\end{array}\right]^{\mathrm{T}}$ and every initial distribution $\mu_{0}^{N}$. This in turn implies that $\lim _{k \rightarrow 0} \mathbf{E}\left\{\|\tilde{x}(k)\|^{2}\right\}=0$ for every initial condition $\left[\begin{array}{ll}x_{0}^{\mathrm{T}} & z_{0}^{\mathrm{T}}\end{array}\right]^{\mathrm{T}}$ and every initial distribution $\mu_{0}^{N}$, making (111) MSS.

(Necessity) The converse argument is completely analogous.

Corollary C.2.1 is the equivalent for HJLS's of Theorem 3.3 in (Patilkulkarni et al. 2004). It shows that the MS stability of (110) can be analyzed uding (111).

\section{C.3 A-EQUIVALENCE OF GENERAL MOORE-TYPE HJLS'S AND CANONICAL HJLS'S}

Chapter II introduced a procedure to build an A-equivalent canonical HJLS for any HJLS with an embedded Moore-Type FSM equipped with a non-isomorphic 
output map. Specifically, it was claimed that the HJLS

$$
\begin{aligned}
\boldsymbol{x}(k+1) & =A_{\varpi(\boldsymbol{z}(k))} \boldsymbol{x}(k) \\
\boldsymbol{z}(k+1) & =S_{\boldsymbol{N}(k), \psi(\boldsymbol{x}(k))} \boldsymbol{z}(k) \\
\psi(x) & =\sum_{i=0}^{\ell_{\nu}-1} i 1_{\left\{x \in R_{i}\right\}}, \quad \bigcup_{i \in \mathcal{I}_{\ell_{\nu}}} R_{i}=\mathbb{R}^{n},
\end{aligned}
$$

with a non-isomorphic output map $\varpi$, and the canonical HJLS

$$
\begin{aligned}
\tilde{\boldsymbol{x}}(k+1) & =\hat{A}_{\boldsymbol{z}(k)} \tilde{\boldsymbol{x}}(k) \\
\boldsymbol{z}(k+1) & =S_{\boldsymbol{N}(k), \psi(\boldsymbol{x}(k))} z(k) \\
\psi(x) & =\sum_{i=0}^{\boldsymbol{\ell}_{\nu}-1} i \mathbf{1}_{\left\{\boldsymbol{x} \in R_{i}\right\}}, \quad \bigcup_{i \in \mathcal{I}_{\boldsymbol{\ell}_{\nu}}} R_{i}=\mathbb{R}^{n},
\end{aligned}
$$

are A-equivalent provided that $\hat{A}_{\boldsymbol{z}(k)}=A_{i}$ whenever $\varpi(\boldsymbol{z}(k))=i$. This claim can be proven as is Lemma C.2.1. More importantly, it is also possible to show that their mean square stabilities are equivalent. To see this, observe that a procedure similar to the one leading to (114) yields

$$
\begin{aligned}
& \mu_{k}^{x}(B)= \\
& \sum_{r_{0}=1}^{\ell_{S}} \sum_{i_{0}=0}^{\ell_{N}-1} \cdots \sum_{r_{k-1}=1}^{\ell_{S}} \sum_{i_{k-1}=0}^{\ell_{N}-1} \mu_{0}^{(\bar{z}, N)}\left(\left\{\left(e_{r_{0}}, i_{0}\right)\right\}\right) \underset{\left(r_{0}, i_{0}\right),\left(r_{1}, i_{1}\right)}{\mathrm{P}}(\bar{x})_{\left(r_{1}, i_{1}\right),\left(r_{2}, i_{2}\right)}\left(A_{\varpi\left(e_{r_{0}}\right)} \bar{x}\right) \cdots \\
& \underset{\left(r_{k-2}, i_{k-2}\right),\left(r_{k-1}, i_{k-1}\right)}{\mathrm{P}}\left(A_{\varpi\left(e_{r_{k-3}}\right)} \cdots A_{\varpi\left(e_{r_{0}}\right)} \bar{x}\right) \delta_{\left.\left.A_{\varpi\left(e_{r_{k-1}}\right.}\right) \cdots A_{\varpi\left(e_{r_{0}}\right)}\right) \bar{x}}(B), \quad \text { (1) }
\end{aligned}
$$

and

$$
\begin{aligned}
& \mu_{k}^{\hat{x}}(B)= \\
& \sum_{r_{0}=1}^{\ell_{S}} \sum_{i_{0}=0}^{\ell_{N}-1} \cdots \sum_{r_{k-1}=1}^{\ell_{S}} \sum_{i_{k-1}=0}^{\ell_{N}-1} \mu_{0}^{(\tilde{z}, N)}\left(\left\{\left(e_{r_{0}}, i_{0}\right)\right\}\right) \underset{\left(r_{0}, i_{0}\right),\left(r_{1}, i_{1}\right)}{\mathrm{P}}(\bar{x}) \underset{\left(r_{1}, i_{1}\right),\left(r_{2}, i_{2}\right)}{\mathrm{P}}\left(\hat{A}_{e_{r_{0}}} \bar{x}\right) \cdots \\
& \underset{\left(r_{k-2}, i_{k-2}\right),\left(r_{k-1}, i_{k-1}\right)}{\mathrm{P}}\left(\hat{A}_{e_{r_{k-3}}} \cdots \hat{A}_{e_{r_{0}}} \bar{x}\right) \delta_{\hat{A}_{e_{r_{k-1}}} \cdots \hat{A}_{e_{0}} \bar{x}}(B) .
\end{aligned}
$$


Since $\hat{A}_{z(k)}=A_{i}$, whenever $\varpi(z(k))=i$, then $\hat{A}_{e_{r_{k}}}=A_{\varpi\left(e_{r_{k}}\right)}$ for every $k \geq 0$. Thus, $\mu_{k}^{x}=\mu_{k}^{\hat{x}}$, which in turn shows that Theorem C.2.1 and Corollary C.2.1 also hold for systems (115) and (116).

Remark C.3.1 The preceding discussion justifies the following conclusion: All the stability results derived for canonical HJLS in Chapters III and IV are also valid for general Moore-type HJLS's. 


\section{ARTURO TEJADA RUIZ}

827 W. 48th St. Apt. B, Norfolk, VA 23508 • 757-489-4941 • atejadar@odu.edu

http://www.lions. odu. edu/ ratejadar/

\section{EDUCATION}

- Ph.D. in Electrical Engineering, December 2006, Old Dominion University, Norfolk, VA. Dissertation Title: Stability Analysis of Hybrid Jump Linear Systems with Markov Inputs Dissertation advisor: Dr. Oscar R. González.

- MSc. in Electrical Engineering, August 2002, Old Dominion University, Norfolk, VA, USA. Thesis title: Analysis of Error Recovery Effects on Digital Flight Control Systems Thesis advisor: Dr. Oscar R. González.

- B.S. in Electrical Engineering, December 1996, Pontifical Catholic University of Peru. Thesis title: Design and Development of the Electronic Control of a Heart-Lung Machine Thesis advisor: Msc. Hugo Pratt Linares.

\section{PROFESSIONAL EXPERIENCE}

- August 2000 - December 2006, ECE Department, Old Dominion University Graduate Research Assistant (2000-2006), Systems Research Laboratory

- Performed research on stochastic hybrid jump linear systems. The research was sponsored by National Science Foundation, NASA Langley Research Center, and Old Dominion University.

- Developed mathematical models of digital control systems deployed on fault-tolerant computers subject. The models were implemented in Matlab and tested through MonteCarlo simulations.

Lecturer (Spring 2006), ECE Department. Delivered course lectures. Supervised laboratory experiments and teaching assistants for ENGN110/111 "Explore Engineering/Tech".

Teaching Assistant (2004-2006), ECE Department. Delivered course lectures, graded homework and reports, and delivered Simulink-based class experiments for

- Fall 2006, ECE 461/561 "Automatic Control Systems"

- Summer 2006, ECE 202 "Circuits, Signals and Linear Systems"

- Fall 2005, ECE 202 "Circuits, Signals and Linear Systems"

- Spring 2005, ECE 651 "Statistical Analysis and Simulation"

- Fall 2004, ECE 461/561 "Automatic Control Systems"

- March 1997 - August 2000, EE Department, Pontifical Catholic University of Peru

Lecturer (1997-200), EE and CS Departments. Created and advised the student Research Group on Microcontrollers. Developed content and class materials for curses such as "Computer Architecture", "Analog Design", and "Digital Circuits and Systems".

Laboratory Manager (2000), EE Department. Manager of the Laboratory of Manufacturing Technologies. Scheduled and supervised the laboratory work. Devised safety procedures and improved infrastructure.

- March 1994 - December 1997, Bioengineering Research Group, Pontifical Catholic University of Peru

Undergraduate Research Assistant (1994-1997). Developed blood pressure and body temperature data acquisition systems. 


\section{PUBLICATIONS AND PRESENTATIONS *}

Journal Publications

1. A. Tejada, O. R. González and W. S. Gray, "Stability of Digital Control Systems implemented in Error-Recoverable Computers," International Journal of Control, under review.

2. A. Tejada, O. R. González and W. S. Gray, "On the Markov Property for Nonlinear DiscreteTime Systems with Markovian Inputs " IEEE Transactions on Automatic Control, under review.

3. O. R. González, W. S. Gray and A. Tejada, "Analytical Tools for the Design and Verification of Safety Critical Control Systems," 2001 SAE Transactions - Journal of Aerospace, vol. 110, Section 1, 2002, pp. 481-490.

Conference Publications

1. A. Tejada, Heber Herencia-Zapana O. R. González and W. S. Gray, "Mean Square Stability Analysis of Hybrid Jump Linear Systems using a Markov Kernel Approach," Proc. 2007 American Control Conference, New York City, New York, 2007, under review.

2. A. Tejada, O. R. González and W. S. Gray, "On the Markov Property for Nonlinear DiscreteTime Systems with Markovian Inputs," Proc. 2006 American Control Conference, Minneapolis, Minnesota, 2006, pp. 899-904.

3. A. Tejada, O. R. González, and W. S. Gray, "A Measure-Theoretic Proof of the Markov Property for Hybrid Systems with Markovian Inputs," Proc. 38th IEEE Southeastern Symposium on System Theory, Cookeville, Tennessee, 2006, pp. 328-332.

4. A. Tejada, O. R. González and W. S. Gray, "Asymptotic and Mean Square Stability Conditions for Hybrid Jump Linear System with Performance Supervision," Proc. 2005 American Control Conference, Portland, Oregon, 2005, pp. 569-574.

5. A. Tejada, O. R. González and W. S. Gray, "Towards Stability Analysis of Jump Linear Systems with State-Dependent and Stochastic Switching," Proc. 2004 American Control Conference, Boston, Massachusetts, pp. 1893-1898, 2004.

6. O. R. González, A. Tejada and W. S. Gray, "Analysis of Design Trade-Offs in the Rollback Recovery Method for Fault Tolerant Digital Control Systems," Proc. 2002 American Control Conference, Anchorage, Alaska, pp. 4801-4806, 2002.

7. O. R. González, W. S. Gray, A. Tejada and S. Patilkulkarni, "Stability Analysis of Electromagnetic Interference Upset Recovery Methods," Proc. 40th IEEE Conference on Decision and Control, Orlando, Florida, pp. 4134-4139, 2001.

8. O. R. González, W. S. Gray, A. Tejada and S. Patilkulkarni, "Stability Analysis of Upset Recovery Methods for Electromagnetic Interference," Proc. 20th DASC Digital Avionics Systems Conference, Daytona Beach, Florida, pp. 1.C.4-1-9, 2001.

9. O. R. Gonzalez, W. S. Gray and A. Tejada, "Analytical Tools for the Design and Verification of Safety Critical Control Systems," Proc. of the 2001 International Conference on Lightning and Static Electricity, Seattle, Washington, 2001 (invited).

Presentations

1. "On the Markov Property for Nonlinear Discrete-Time Systems with Markovian Inputs," 2006 American Control Conference, Minneapolis, Minnesota, June 14, 2006.

2. "A Measure-Theoretic Proof of the Markov Property for Hybrid Systems with Markovian Inputs," 38th Southeastern Symposium on System Theory, Cookeville, Tennessee, March 6, 2006.

3. "Asymptotic and Mean Square Stability Conditions for Hybrid Jump Linear Systems with Performance Supervision," 2005 American Control Conference, Portland, Oregon, June 8, 2005.

4. "Towards Stability Analysis of Jump Linear Systems with State-Dependent and Stochastic Switching," 2004 American Control Conference, Boston, Massachusetts, June 30, 2004.

*Electronic copies of these publications can be downloaded from http://www.lions.odu.edu/ -sgray/publ.html, using the word 'guest' as both login and password. 


\section{ACADEMIC AWARDS}

- Best Paper in Session Award for "On the Markov Property for Nonlinear Discrete-Time Systems with Markovian Inputs," (with O. R. González and W. S. Gray), 2006 American Control Conference, Minneapolis, 2006

- 2006 Outstanding Graduate Teaching Assistant Award, ECE Department, Old Dominion University

- Recipient of the Student Travel Grant for the American Control Conference in 2004 and 2005

- Supplemental Dissertation Stipend Award (USD 5500), Old Dominion University, 2005

- Outstanding Graduate Research Master Student Award, ECE Department, ODU, 2002

- Best Paper in Session Award for "Analysis of Design Tradeoffs in Rollback Recovery Methods for Fault Tolerant Digital Control Systems," (with O. R. González and W. S. Gray), 2002 American Control Conference, Anchorage, Alaska, 2002

- Best SAE Technical Paper in 2001 for "Analytical Tools for the Design and Verification of Safety Critical Control Systems," (with O. R. González and W. S. Gray), SAE Transactions - Journal of Aerospace, Vol. 110, Section 1, 2002, pp. 481-490

- Presidential Dominion Scholar Fellowship, Old Dominion University (USD 18000), 2000-2002

- Best Project Design, Peruvian National Congress on Mechanical and Electrical Engineering and Related Fields (CONIMERA'99), 1999

\section{PROFESSIONAL ACTIVITIES}

- Co-Chair, Nonlinear Systems Theory session (WeC03), 2006 American Control Conference

- Technical reviewer for IEEE Transactions on Circuits and Systems I, 2005

- Technical reviewer for the $44^{\text {th }}$ IEEE Conference on Decision and Control, 2005 (CDC'05)

- Technical reviewer for the $\mathbf{4 3}^{\text {rd }}$ IEEE Conference on Decision and Control, 2004 (CDC'04)

\section{PROFESSIONAL AFFILIATION}

- Student member of the IEEE and IEEE Control Systems Society.

\section{LANGUAGE SKILLS}

- Spanish (native)

- English 